\title{
Formation of Substituted Alkyls as Precursors of Peroxy Radicals with a Rapid H-shift in the Atmosphere
}

Xiaoqing $W u^{a, b}$, Can Huang ${ }^{c}$, Jiajue Chai ${ }^{d}$ and Feng Zhang ${ }^{*}, a$

a. Hefei National Laboratory for Physical Sciences at the Microscale, University of Science and Technology of China, Hefei, Anhui, 230026, P. R. China.

b. National Synchrotron Radiation Laboratory, University of Science and Technology of China, Hefei, Anhui, 230029, P. R. China.

c. Chair of Technical Thermodynamics, RWTH Aachen University, 52062 Aachen, Germany.

d. Institute at Brown for Environment and Society, and Department of Earth, Environmental and Planetary Sciences, Brown University, 182 Hope St., Providence, RI, 02912, USA

\section{Table of Contents}

- Section S1. The method of energy and rate coefficient calculations

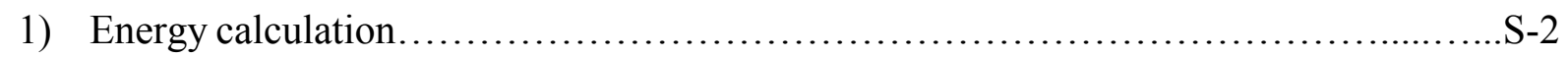

2) Rate coefficient calculation...................................................

- Section S2. The calculated results based on the theoretical computation in Section 1

1) Table S1. Structure and frequency of the stationary points........................

2) Table S2. The structures of various transition states, reaction complexes(RCs) and product complexes (PCs) on 3-MH + $\mathrm{OH}$ and 2-MP $+\mathrm{OH}$ potential energy surface.

3) Table S3. T1 diagnostic, spin expectation values and point group of the stationary points.

4) Table S4. Final rate coefficients $\left(\mathrm{cm}^{3}\right.$ molecule $\left.{ }^{-1} \mathrm{~s}^{-1}\right)$ of various channels at 1.0 atm...S-53

5) Table S5. The computed RRKM/ME rate coefficients $\left(\mathrm{cm}^{3}\right.$ molecule $\left.{ }^{-1} \mathrm{~s}^{-1}\right)$ in the format of $\mathrm{k}=\mathrm{A} \cdot \mathrm{T}^{\mathrm{n}} \cdot \exp \left(-\mathrm{E}_{\mathrm{a}} / \mathrm{RT}\right)$ at $200-2000 \mathrm{~K}, 1 \mathrm{~atm}$. 57 
6) Table S6. The total rate coefficients $\left(\mathrm{cm}^{3}\right.$ molecule $\left.{ }^{-1} \mathrm{~s}^{-1}\right)$ based on the previous experiments, theories, and structure-activity relationship (SAR) model under atmospheric conditions. . .

7) Table S7. Final total rate coefficients $\left(\mathrm{cm}^{3}\right.$ molecule $\left.{ }^{-1} \mathrm{~s}^{-1}\right)$ at $0.1,0.4,0.7,1.0,1.2,2.0$, $4.0,6.0,8.0,10 \mathrm{~atm}, 200-2000 \mathrm{~K}$ S-63

8) Figure $\mathrm{S} 1$. The branching ratios of various reaction channels at $1.0 \mathrm{~atm}, 200-2000 \mathrm{~K} . . \mathrm{S}-66$

9) Figure S2. Final total rate coefficients $\left(\mathrm{cm}^{3}\right.$ molecule $\left.{ }^{-1} \mathrm{~s}^{-1}\right)$ at $0.1,0.4,0.7,1.0,1.2,2.0$, $\begin{array}{lllll}4.0, & 6.0, & 8.0, & 10 & \mathrm{~atm},\end{array}$ $2000 \mathrm{~K}$ S-67

10) Figure $S 3$. The rate coefficients of various reaction channels at $0.1,0.4,0.7,1.0,1.2,2.0$, $4.0,6.0,8.0,10 \mathrm{~atm}, 200-2000 \mathrm{~K}$. S-68

11) Figure $S 4$. The branching ratios of various reaction channels at $0.1,0.4,0.7,1.0,1.2,2.0$, $4.0,6.0,8.0,10 \mathrm{~atm}, 200-2000 \mathrm{~K}$

12) Figure $\mathrm{S} 5$. The rate coefficients of $\mathrm{OH}$ addition, $\mathrm{H}$ abstraction and addition-dissociation reactions at $0.1,0.4,0.7,1.0,1.2,2.0,4.0,6.0,8.0,10 \mathrm{~atm}, 200-2000 \mathrm{~K}$ S-76

13) Figure $\mathrm{S} 6$. The branching ratios of $\mathrm{OH}$ addition, $\mathrm{H}$ abstraction and addition-dissociation reactions at $0.1,0.4,0.7,1.0,1.2,2.0,4.0,6.0,8.0,10 \mathrm{~atm}, 200-2000 \mathrm{~K}$

14) Figure $S 7$. The ratio of the rate coefficient $(R->$ abs 1$)$ at 0.1 atm to that at 10 atm with the shift of $\mathrm{RC}_{\mathrm{abs} 1}$ energy. S-79

15) Figure $\mathrm{S} 8$. Ratios of rate coefficients for $3-\mathrm{MH}+\mathrm{OH}$ addition channels with or without the pre-reaction complex. S-80

16) Figure $S 9$. The total rate coefficients after the barrierenergy shift of $\pm 0.2 \mathrm{kcal} / \mathrm{mol} \ldots . . \mathrm{S}-80$

17) Figure S10. The branching ratios after the barrierenergy shift of $\pm 0.2 \mathrm{kcal} / \mathrm{mol}$ S-82

\section{- Section S1. The method of energy and rate coefficient calculations}

\section{1) Energy calculation}

The structural optimization and vibrational analysis on 3-methyl-1-hexene (3-MH)+ OH and 2-methylpentanal (2-MP)+ OH potential energy surface (PES) were calculated by the density functional method of M06-2 $\mathrm{x}^{1}$ with Dunning's augmented cc-pVTZ basis set, which has been successfully applied in toluene $+\mathrm{OH}$ and xylene $+\mathrm{OH} \cdot{ }^{2-4}$ The vibrational analysis was 
employed at the same theoretical level to characterize the nature of each critical point on the PES. An intrinsic reaction coordinate (IRC) calculation was also performed for each reaction path in this work. The single point energies were determined by the $\operatorname{CCSD(T)/aug-cc-pVTZ~}$ method. Sirianni et al. ${ }^{5}$ mentioned that the aTZ or aQZ basis sets along with coupled cluster calculations could obtain results closer to the CBS limit. The mean absolute errors (MAEs) for $\operatorname{CCSD}$ (T)/aug-cc-pVTZ is $\sim 0.12 \mathrm{kcal} / \mathrm{mol}$ for the complex with hydrogen bonding 5 . Then bond dissociation energy (BDE) calculations including zero-point energies were based on the above theoretical calculation (CCSD(T)/aug-cc-pVTZ//M06-2x/cc-pVTZ).

The scale factors determined for M06-2x/cc-pVTZ are 0.9480 for harmonic vibrational frequencies and 0.9710 for zero-point vibrational energies. ${ }^{6}$ The $\mathrm{T} 1$ diagnostics at the CCSD(T)/aug-cc-pVTZ level for stationary points are computed in Table S3. T1 diagnostic values of closed-shell species are all lower than 0.02 and those of open-shell species are all lower than 0.045 , indicating insignificant multireference characteristics were involved in the studied reaction system. ${ }^{7-8}$ The spin expectation values $\left(<\mathrm{S}^{2}>\right)$ demonstrate that the wave function is not seriously contaminated by the high multiplicity. ${ }^{9}$ Most of the quantum chemical calculations in this work are conducted by Gaussian $16^{10}$ and the $\operatorname{CCSD}(\mathrm{T})$ calculations are performed with the ORCA program package. ${ }^{11-12}$ In addition, a molecular dynamics simulation based on the GFN2-xTB method ${ }^{13}$ was used to screen reasonable configurations of 3-MH + $\mathrm{OH}$ and 2-MP $+\mathrm{OH}$ PES by xtb-6.4.0. The Molclus program ${ }^{14}$ was employed to search the structures on the trajectory of molecular dynamics with default threshold.

\section{2) Rate coefficient calculation}

The Rice-Ramsperger-Kassel-Marcus/master equation (RRKM/ME) method ${ }^{15-17}$ was performed to compute the rate coefficients for various reaction channels of 3-MH $+\mathrm{OH}$ and 2$\mathrm{MP}+\mathrm{OH}$. We focused on the results at the region of 200-450K, 0.1-10atm, covering almost all atmospheric conditions. We also provided the rate coefficients over a wide range of temperature $(450-2000 \mathrm{~K})$ and pressure $(0.1-10 \mathrm{~atm})$ for the potential usefulness of kinetic modeling. The RRKM/ME approach provides a theoretical description and solution to evaluate the phenomenological rate coefficients, which involves coupling of collision-induced energy transfer and microscopic chemical transformations. The eigenvalue-eigenvector (matrix) 
technique was adopted to solve the one-dimensional master equation in this work using the MESS code. ${ }^{15,18}$ The $|\mathrm{f}\rangle$ described the time evolution of the nonequilibrium vector in master equation ${ }^{15}$ is expressed as equation 1 . In which, the $|\mathrm{f}\rangle$ is the nonequilibrium and instantaneous statistical state of the reactive complex. $\hat{G}$ is the kinetic relaxation operator. $\mathrm{s}_{\mathrm{v}}(\mathrm{t})$ is relative to the concentrations of the V-th bimolecular reactants and standard partition function $\mathrm{Q}_{v} \cdot \mathrm{Q}_{v}$ is the standard partition function for the relative and internal motions of the reactants. $\mathrm{p}^{(v)}$ is the time-independent vector, which represents the microscopic population gain from the $v$-th pair of bimolecular reactants.

$$
\begin{gathered}
\frac{d}{d t}|f\rangle=-\hat{G}|f\rangle+\sum_{\nu} s_{v}(t)\left|p^{(v)}\right\rangle \\
k_{\nu \rightarrow \mu}=\frac{1}{Q_{v}} \sum_{\lambda} \frac{1}{\Lambda_{\lambda}} p_{\lambda}^{(\mu)} p_{\lambda}^{(v)}
\end{gathered}
$$

The phenomenological rate coefficients can be compacted as equation $2 . \Lambda_{\lambda}$ is the eigenvalue associated with the $\lambda$ eigenvector. $\mathrm{p}_{\lambda}^{(\mu)}$ and $\mathrm{p}_{\lambda}{ }^{(v)}$ explain the microscopic population from the $\mu$ th and $v$-th pair with the $\lambda$ eigenvector. The master equation provides a correlation for phenomenological rate coefficients and microscopic dynamics directly and explains the time dependence of the energy resolved populations. ${ }^{15,}$ 19-20 The rate coefficients for a multi-well and multi-channel reaction system are complex and not easily related to the solution of the master equation. ${ }^{16,21}$ Such correlation depends on the separation of eigenvalues from the transition matrix between slow chemical processes (chemically significant eigenvalues, CSEs) and those that describe fast energy relaxation (internal energy relaxation eigenvalues, IEREs). ${ }^{17}$ At high temperature, due to the disappearance of time scale separation for some of the CSEs, chemical species tend to merge together and the phenomenological rate constants are difficult to be defined. ${ }^{16-17}$ The numerical dispersion in the eigenvalues could also lead to numerical limitation in the calculation results in low temperature region. ${ }^{16}$

We treated the torsion modes of methyl and hydroxyl as one-dimensional hindered rotors (HR) and the hindrance potentials were calculated by M06-2x/cc-pVTZ. The microcanonical variational transition state theory ( $\mu \mathrm{VTST}$ ) was performed to calculate the rate coefficients for the reaction barrier was lower than $2 \mathrm{kcal} / \mathrm{mol}$ and the non-barrier reaction, with a grid of $\sim 0.05$ 
115 Bohr. The quantum tunneling was simulated by the Eckart tunneling model. ${ }^{22}$ The collision 116 between reaction complex and bath gas- $\mathrm{N}_{2}$ was simulated by the Lennard-Jones (L-J) potential. 117 The L-J parameters were provided as $\sigma=3.9 \AA$ and $\varepsilon=48 \mathrm{~cm}^{-1}$ for $\mathrm{N}_{2}$, and $\sigma=6.70 \AA$ and $\varepsilon=$ $118524.33 \mathrm{~cm}^{-1}$ for the reaction complex in analogy to 4-methyl-5-hexen-1-ol for 3-MH $+\mathrm{OH}(\sigma=$

$6.47 \AA$ and $\varepsilon=579.53 \mathrm{~cm}^{-1}$ for the reaction complex in analogy to 4-hydroxy-2-methylpentanal for 2-MP $+\mathrm{OH})$. The single-exponential down model was used to mimic collisional energy transfer by $<\Delta \mathrm{E}>_{\text {down }}=240 \times(T / 298)^{1.00} \mathrm{~cm}^{-1}$ (an analogy for $\mathrm{n}$-hexene ${ }^{23}$ for 3-MH + $\mathrm{OH})$ and $\langle\Delta \mathrm{E}\rangle_{\text {down }}=200 \times(T / 298)^{0.85} \mathrm{~cm}^{-1}$ (an analogy for iso-butanol ${ }^{24}$ for 2-MP + $\mathrm{OH})$.

\section{- Section S2. The calculated results based on the theoretical computation in Section 1}

1) Table S1. Structure and frequency of all stationary points shown on the 3-methyl-1-hexene $(3-\mathrm{MH})+\mathrm{OH}$ and 2-methylpentanal(2-MP) + OH PES optimized by the M06-2X/cc-pVTZ method.

\begin{tabular}{|c|c|c|c|c|}
\hline \multicolumn{5}{|l|}{ 3-MH } \\
\hline $\mathrm{C}$ & & 1.88354300 & 1.79429500 & -0.11182800 \\
\hline $\mathrm{H}$ & & 2.29901000 & 2.59772600 & 0.48114800 \\
\hline $\mathrm{H}$ & & 1.88614600 & 1.92161300 & -1.18810700 \\
\hline $\mathrm{C}$ & & 1.39164100 & 0.69926600 & 0.44622500 \\
\hline $\mathrm{H}$ & & 1.40238300 & 0.60302000 & 1.53070400 \\
\hline $\mathrm{C}$ & & 0.80829400 & -0.46892000 & -0.29227500 \\
\hline $\mathrm{C}$ & & -0.65504500 & -0.69520000 & 0.10374900 \\
\hline $\mathrm{H}$ & & -0.70336800 & -0.88678800 & 1.18175200 \\
\hline $\mathrm{H}$ & & -1.01212500 & -1.60653900 & -0.38530800 \\
\hline $\mathrm{C}$ & & -1.58114000 & 0.46141100 & -0.24901500 \\
\hline $\mathrm{H}$ & & -1.50486800 & 0.66467000 & -1.32082700 \\
\hline $\mathrm{H}$ & & -1.24072300 & 1.36677600 & 0.25707900 \\
\hline $\mathrm{C}$ & & -3.03085900 & 0.17425900 & 0.12024400 \\
\hline $\mathrm{H}$ & & -3.12849000 & -0.00973800 & 1.19128200 \\
\hline $\mathrm{H}$ & & -3.68323900 & 1.00833400 & -0.13600000 \\
\hline $\mathrm{H}$ & & -3.39835400 & -0.71109400 & -0.40088900 \\
\hline $\mathrm{C}$ & & 1.63579000 & -1.72620900 & -0.01943500 \\
\hline $\mathrm{H}$ & & 1.22721700 & -2.58285200 & -0.55652400 \\
\hline $\mathrm{H}$ & & 2.67274000 & -1.58843200 & -0.32357600 \\
\hline $\mathrm{H}$ & & 1.62580700 & -1.96294700 & 1.04665500 \\
\hline $\mathrm{H}$ & & 0.84451900 & -0.24716300 & -1.36337300 \\
\hline \multicolumn{5}{|c|}{ Frequencies $[1 / \mathrm{cm}]$} \\
\hline 67.24 & 398.05 & $944.34 \quad 1227.48$ & $1407.61 \quad 289$ & $06 \quad 2974.46$ \\
\hline
\end{tabular}




\begin{tabular}{|c|c|c|c|c|c|c|c|}
\hline 154 & 77.07 & 494.69 & 988.53 & 1248.18 & 1419.17 & 2895.29 & $9 \quad 2994.97$ \\
\hline 155 & 110.67 & 671.23 & 992.35 & 1262.12 & 1421.48 & 2898.70 & $0 \quad 3075.68$ \\
\hline 156 & 170.21 & 709.40 & 1006.05 & 1278.16 & 1424.91 & 2908.40 & \\
\hline 57 & 224.28 & 782.98 & 1026.64 & 1285.36 & 1426.54 & 2934.32 & \\
\hline 158 & 233.23 & 834.00 & 1079.32 & 1325.10 & 1434.56 & 2959.56 & \\
\hline 159 & 278.82 & 861.55 & 1120.62 & 1336.72 & 1646.86 & 2962.67 & \\
\hline 160 & 310.09 & 914.62 & 1153.98 & 1341.02 & 2869.88 & 2969.94 & \\
\hline 161 & 339.58 & 919.36 & 1196.80 & 1385.30 & 2887.80 & 2970.07 & \\
\hline 162 & & & & & & & \\
\hline 163 & \multicolumn{7}{|l|}{$\mathrm{OH}$} \\
\hline 164 & \multicolumn{2}{|l|}{$\mathrm{O}$} & \multicolumn{2}{|c|}{0.00000000} & \multicolumn{2}{|c|}{0.00000000} & 0.10793600 \\
\hline 165 & \multicolumn{2}{|l|}{$\mathrm{H}$} & \multicolumn{2}{|c|}{0.00000000} & \multicolumn{2}{|c|}{0.00000000} & -0.86348600 \\
\hline 166 & \multicolumn{4}{|c|}{ Frequencies[1/cm] } & & & \\
\hline 167 & \multicolumn{4}{|l|}{3579.65} & & & \\
\hline 168 & & & & & & & \\
\hline 169 & \multicolumn{4}{|c|}{ RC-add \#pre-reaction complex } & & & \\
\hline 170 & \multicolumn{2}{|l|}{$\mathrm{C}$} & \multicolumn{2}{|c|}{-2.75913900} & \multicolumn{2}{|c|}{0.12355100} & 0.04559100 \\
\hline 171 & \multicolumn{2}{|l|}{$\mathrm{H}$} & \multicolumn{2}{|c|}{-3.65615000} & \multicolumn{2}{|c|}{-0.02646800} & 0.54012600 \\
\hline 172 & \multicolumn{2}{|l|}{$\mathrm{H}$} & \multicolumn{2}{|c|}{-2.83682300} & \multicolumn{2}{|c|}{-0.02922300} & -1.11453700 \\
\hline 173 & \multicolumn{2}{|l|}{$\mathrm{C}$} & \multicolumn{2}{|c|}{-1.61228300} & \multicolumn{2}{|c|}{0.49355600} & 0.51540900 \\
\hline 174 & $\mathrm{H}$ & & -1.57 & 422400 & 0.6535 & 5600 & 1.59192500 \\
\hline 175 & $\mathrm{C}$ & & -0.33 & 105100 & 0.74917 & 400 & -0.22025100 \\
\hline 176 & $\mathrm{H}$ & & -0.48 & 078100 & 0.46672 & 2500 & -1.26637800 \\
\hline 177 & $\mathrm{C}$ & & 0.7 & 294000 & -0.12077 & 900 & 0.35547900 \\
\hline 178 & $\mathrm{H}$ & & 0.4 & 3328200 & -1.1660 & 100 & 0.28618000 \\
\hline 179 & $\mathrm{H}$ & & 0.9 & 919800 & 0.1084 & 6300 & 1.42121700 \\
\hline 180 & $\mathrm{C}$ & & 2.13 & 889000 & 0.0423 & 3600 & -0.34217800 \\
\hline 181 & $\mathrm{H}$ & & 2.5 & 236300 & 1.0520 & 2200 & -0.18546500 \\
\hline 182 & $\mathrm{H}$ & & 1.9 & 963000 & $-0.0745^{\circ}$ & 7200 & -1.42048000 \\
\hline 183 & $\mathrm{C}$ & & 3.16 & 374700 & -0.96881 & 900 & 0.15702000 \\
\hline 184 & $\mathrm{H}$ & & 2.8 & 2362600 & -1.9884 & 4000 & -0.02799800 \\
\hline 185 & $\mathrm{H}$ & & 4.12 & 2719000 & $-0.8410^{\prime}$ & 7100 & -0.33530600 \\
\hline 186 & $\mathrm{H}$ & & 3.3 & 2023300 & -0.86217 & 7200 & 1.23167300 \\
\hline 187 & $\mathrm{C}$ & & 0.00 & 833100 & 2.2411 & 8600 & -0.15171200 \\
\hline 188 & $\mathrm{H}$ & & 0.2 & 816900 & 2.5367 & 7400 & 0.87913400 \\
\hline 189 & $\mathrm{H}$ & & 0.8 & 3407400 & 2.4763 & 5200 & -0.75491900 \\
\hline 190 & $\mathrm{H}$ & & -0.82 & 438100 & 2.8435 & 200 & -0.51297000 \\
\hline 191 & $\mathrm{O}$ & & -1.81 & 556600 & -2.27819 & 600 & -0.32349900 \\
\hline 192 & $\mathrm{H}$ & & -1.79 & 948600 & -2.28511 & 200 & 0.64672900 \\
\hline 193 & Frequencies[ & /cm] & & & & & \\
\hline 194 & 25.05 & 236.45 & 796.10 & 1042.36 & 1287.52 & 1430.28 & $8 \quad 2940.27$ \\
\hline 195 & 63.24 & 300.94 & 831.90 & 1084.13 & 1326.33 & 1434.49 & 2962.67 \\
\hline 196 & 75.76 & 313.95 & 862.77 & 1124.44 & 1334.93 & 1624.75 & $5 \quad 2964.38$ \\
\hline 197 & 82.79 & 337.85 & 910.07 & 1149.28 & 1340.94 & 2869.30 & 2968.28 \\
\hline 198 & 110.28 & 386.97 & 926.04 & 1199.28 & 1385.37 & 2891.95 & $5 \quad 2972.16$ \\
\hline 199 & 146.23 & 416.53 & 939.51 & 1228.33 & 1408.79 & 2894.78 & $8 \quad 2980.35$ \\
\hline
\end{tabular}




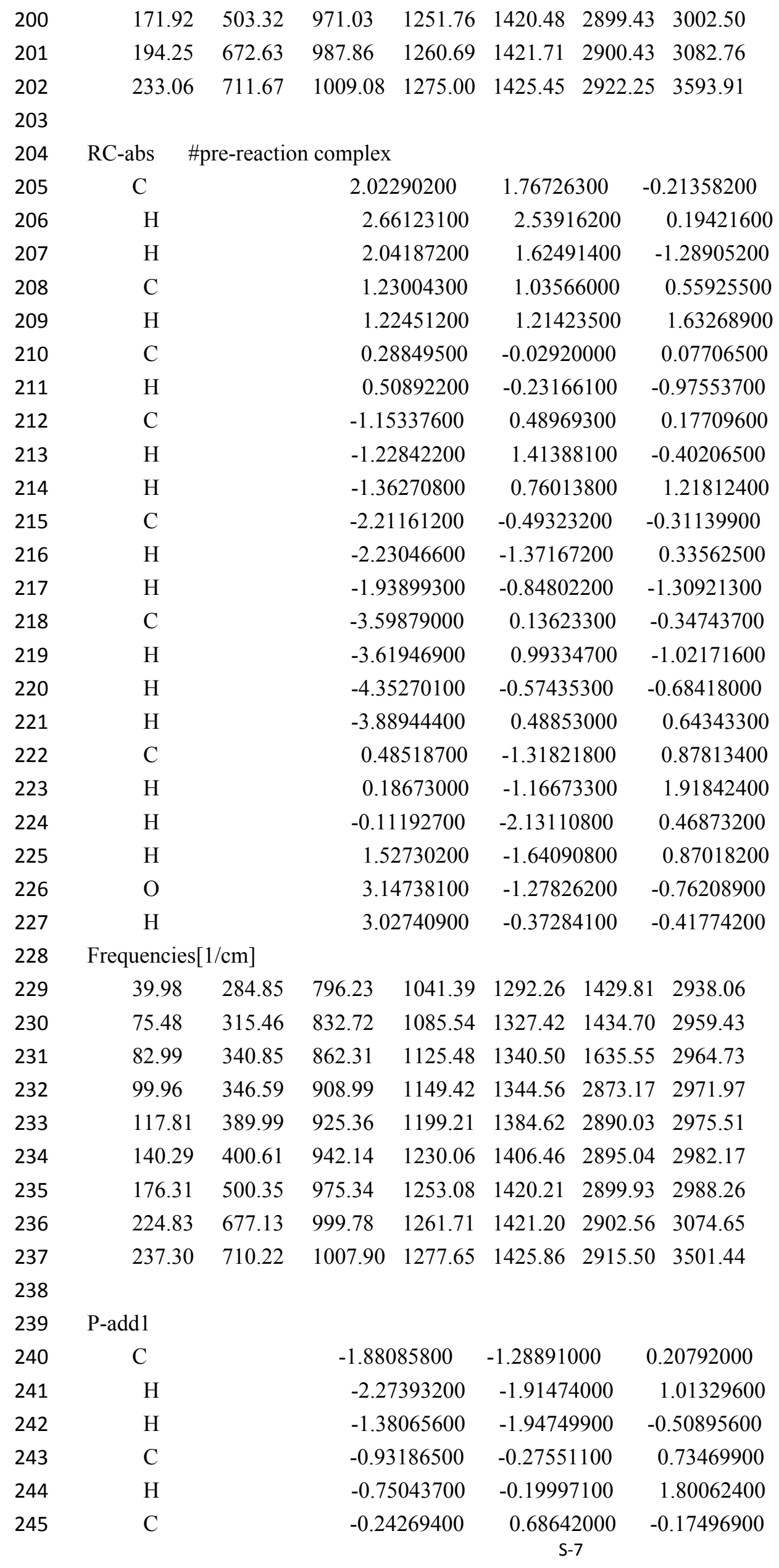




\begin{tabular}{|c|c|c|c|c|c|}
\hline 246 & $\mathrm{H}$ & & -0.43882600 & 0.37255200 & -1.20528900 \\
\hline 247 & $\mathrm{C}$ & & 1.27415900 & 0.68229500 & 0.05289800 \\
\hline 248 & $\mathrm{H}$ & & 1.47609400 & 0.97230400 & 1.09022600 \\
\hline 249 & $\mathrm{H}$ & & 1.72956500 & 1.45236500 & -0.57697100 \\
\hline 250 & $\mathrm{C}$ & & 1.93385200 & -0.66015000 & -0.23683700 \\
\hline 251 & $\mathrm{H}$ & & 1.70661400 & -0.95426000 & -1.26524000 \\
\hline 252 & $\mathrm{H}$ & & 1.49302600 & -1.42597200 & 0.40644200 \\
\hline 253 & $\mathrm{C}$ & & 3.44279000 & -0.62284000 & -0.03239800 \\
\hline 254 & $\mathrm{H}$ & & 3.90114500 & -1.58866400 & -0.24221200 \\
\hline 255 & $\mathrm{H}$ & & 3.90554500 & 0.11696400 & -0.68724500 \\
\hline 256 & $\mathrm{H}$ & & 3.68760200 & -0.35213800 & 0.99579100 \\
\hline 257 & $\mathrm{C}$ & & -0.80612500 & 2.10215800 & 0.00170700 \\
\hline 258 & $\mathrm{H}$ & & -0.65887500 & 2.44374700 & 1.02824200 \\
\hline 259 & $\mathrm{H}$ & & -0.30553000 & 2.80575100 & -0.66518200 \\
\hline 260 & $\mathrm{H}$ & & -1.87371000 & 2.12386400 & -0.21549700 \\
\hline 261 & $\mathrm{O}$ & & -2.94602000 & -0.69782100 & -0.53982500 \\
\hline 262 & $\mathrm{H}$ & & -3.38501500 & -0.06249800 & 0.03244700 \\
\hline 263 & \multicolumn{5}{|c|}{ Frequencies $[1 / \mathrm{cm}]$} \\
\hline 264 & 11.73 & 312.82 & $857.93 \quad 1109.19$ & $1318.81 \quad 1425.99$ & 92905.74 \\
\hline 265 & 67.52 & 333.84 & $905.86 \quad 1121.07$ & $1328.72 \quad 1428.22$ & $22 \quad 2913.92$ \\
\hline 266 & 77.71 & 359.50 & $921.41 \quad 1154.27$ & $1335.81 \quad 1433.78$ & $\begin{array}{l}82926.96\end{array}$ \\
\hline 267 & 117.04 & 411.10 & $951.90 \quad 1192.88$ & $1341.29 \quad 2869.65$ & $55 \quad 2958.51$ \\
\hline 268 & 152.46 & 467.41 & $983.14 \quad 1227.27$ & $1360.20 \quad 2877.41$ & $11 \quad 2961.67$ \\
\hline 269 & 212.90 & 579.63 & $994.29 \quad 1243.51$ & $1407.15 \quad 2886.20$ & $20 \quad 2970.31$ \\
\hline 270 & 232.64 & 706.09 & $1019.99 \quad 1264.79$ & $1418.18 \quad 2890.63$ & $33 \quad 2970.89$ \\
\hline 271 & 238.84 & 779.02 & $1051.12 \quad 1275.08$ & $1421.86 \quad 2893.25$ & $25 \quad 3011.69$ \\
\hline 272 & 249.08 & 832.69 & $1068.66 \quad 1294.69$ & $1423.20 \quad 2898.65$ & $55 \quad 3665.92$ \\
\hline \multicolumn{6}{|l|}{273} \\
\hline 274 & TS-add1 & & & & \\
\hline 275 & $\mathrm{C}$ & & -1.54316100 & -1.51962600 & 0.04327800 \\
\hline 276 & $\mathrm{H}$ & & -1.98250000 & -2.27825300 & 0.67436900 \\
\hline 277 & $\mathrm{H}$ & & -1.52276400 & -1.71613000 & -1.01924300 \\
\hline 278 & $\mathrm{C}$ & & -0.89295700 & -0.46333400 & 0.56105300 \\
\hline 279 & $\mathrm{H}$ & & -0.87052000 & -0.33186800 & 1.64169300 \\
\hline 280 & $\mathrm{C}$ & & -0.22282300 & 0.60758200 & -0.23852800 \\
\hline 281 & $\mathrm{H}$ & & -0.33645400 & 0.35920900 & -1.29757400 \\
\hline 282 & $\mathrm{C}$ & & 1.27369400 & 0.67869400 & 0.09069200 \\
\hline 283 & $\mathrm{H}$ & & 1.39199900 & 0.90235800 & 1.15692500 \\
\hline 284 & $\mathrm{H}$ & & 1.70572400 & 1.52592000 & -0.44966000 \\
\hline 285 & $\mathrm{C}$ & & 2.04789500 & -0.58717100 & -0.25409700 \\
\hline 286 & $\mathrm{H}$ & & 1.89260600 & -0.82314600 & -1.31044900 \\
\hline 287 & $\mathrm{H}$ & & 1.63997500 & -1.42908200 & 0.30947200 \\
\hline 288 & $\mathrm{C}$ & & 3.53789000 & -0.44866200 & 0.03053500 \\
\hline 289 & $\mathrm{H}$ & & 4.08041000 & -1.35999400 & -0.21824900 \\
\hline 290 & $\mathrm{H}$ & & 3.97058300 & 0.36793100 & -0.54929700 \\
\hline 291 & $\mathrm{H}$ & & 3.71248900 & $\begin{array}{c}-0.23255700 \\
\text { S-8 }\end{array}$ & 1.08563100 \\
\hline
\end{tabular}




\begin{tabular}{|c|c|c|c|c|c|c|c|}
\hline 292 & \multicolumn{2}{|l|}{$\mathrm{C}$} & \multicolumn{2}{|c|}{-0.89834000} & \multicolumn{2}{|c|}{1.95573900} & 0.02453100 \\
\hline 293 & \multicolumn{2}{|l|}{$\mathrm{H}$} & \multicolumn{2}{|c|}{-0.82121900} & \multicolumn{2}{|c|}{2.21966900} & 1.08184400 \\
\hline 294 & \multicolumn{2}{|l|}{$\mathrm{H}$} & \multicolumn{2}{|c|}{-0.41491100} & \multicolumn{2}{|c|}{2.74454700} & -0.55255000 \\
\hline 295 & \multicolumn{2}{|l|}{$\mathrm{H}$} & \multicolumn{2}{|c|}{-1.95132600} & \multicolumn{2}{|c|}{1.91324300} & -0.24752400 \\
\hline 296 & \multicolumn{2}{|l|}{$\mathrm{O}$} & \multicolumn{2}{|c|}{-3.35299100} & \multicolumn{2}{|c|}{-0.38946400} & -0.30675100 \\
\hline 297 & \multicolumn{2}{|l|}{$\mathrm{H}$} & \multirow{2}{*}{\multicolumn{2}{|c|}{-3.48335600}} & -0.08547200 & \multicolumn{2}{|c|}{0.60382900} \\
\hline 298 & \multicolumn{2}{|c|}{ Frequencies[1/cm] } & & & & & \\
\hline 299 & -323.63 & 246.29 & 784.92 & 1025.05 & 1280.18 & 1432.98 & $8 \quad 2931.78$ \\
\hline 300 & 38.27 & 309.17 & 834.56 & 1074.92 & 1321.97 & \multicolumn{2}{|c|}{$\begin{array}{ll}1434.51 \quad 2959.00\end{array}$} \\
\hline 301 & 67.32 & 322.68 & 862.19 & 1117.69 & 1332.21 & \multicolumn{2}{|c|}{$\begin{array}{ll}1561.15 \quad 2962.46\end{array}$} \\
\hline 302 & 88.87 & 343.62 & 917.55 & 1155.22 & 1340.71 & \multicolumn{2}{|c|}{$2871.91 \quad 2970.30$} \\
\hline 303 & 107.19 & 422.95 & 925.62 & 1196.39 & 1385.58 & \multicolumn{2}{|c|}{$2892.07 \quad 2971.11$} \\
\hline 304 & 143.18 & 492.23 & 943.21 & 1227.94 & 1407.17 & \multicolumn{2}{|c|}{$2893.54 \quad 2988.04$} \\
\hline 305 & 178.94 & 651.85 & 961.98 & 1241.35 & 1418.29 & \multicolumn{2}{|c|}{$2898.57 \quad 3015.45$} \\
\hline 306 & 208.28 & 696.80 & 987.53 & 1256.68 & 1420.85 & 2899.88 & $8 \quad 3100.04$ \\
\hline 307 & \multirow[t]{2}{*}{237.07} & 720.84 & 1004.60 & 1274.15 & 1426.15 & 2910.75 & $5 \quad 3603.67$ \\
\hline 308 & & & & & & & \\
\hline 309 & P-diss1 & & & & & & \\
\hline 310 & $\mathrm{C}$ & & 1.45 & 109700 & -0.57000 & 200 & 0.03125800 \\
\hline 311 & $\mathrm{H}$ & & 1.9 & 0200000 & -1.1279 & 3400 & -0.79837200 \\
\hline 312 & $\mathrm{H}$ & & & 2528500 & -1.026 & 12900 & 0.95018500 \\
\hline 313 & $\mathrm{C}$ & & -0.0 & 130900 & -0.7118 & 3500 & -0.03369800 \\
\hline 314 & $\mathrm{H}$ & & -0.4 & 889600 & -1.7256 & 1500 & -0.16893000 \\
\hline 315 & $\mathrm{C}$ & & -0.90 & 222100 & 0.2905 & 1300 & 0.05900100 \\
\hline 316 & $\mathrm{H}$ & & -0.5 & 435800 & 1.2923 & 5000 & 0.21501000 \\
\hline 317 & $\mathrm{C}$ & & -2.38 & 957400 & 0.1460 & 0200 & -0.01221300 \\
\hline 318 & $\mathrm{H}$ & & $-2.6^{\prime}$ & 965100 & -0.8939 & 9200 & -0.15525900 \\
\hline 319 & $\mathrm{H}$ & & -2.8 & 5947600 & 0.5107 & 4300 & 0.90285400 \\
\hline 320 & $\mathrm{H}$ & & -2.8 & 198300 & 0.7333 & 0400 & -0.83460800 \\
\hline 321 & $\mathrm{O}$ & & 1.9 & 0439700 & 0.764 & 57200 & 0.04726600 \\
\hline 322 & $\mathrm{H}$ & & 1.5 & 9394300 & 1.192 & 92500 & -0.75509400 \\
\hline
\end{tabular}

323 Frequencies $[1 / \mathrm{cm}]$

$\begin{array}{lrrr}139.30 & 940.84 & 1309.22 & 2922.23 \\ 192.80 & 952.53 & 1338.46 & 2947.33 \\ 206.44 & 971.09 & 1349.65 & 2974.12 \\ 235.18 & 1022.93 & 1407.15 & 2993.13 \\ 315.65 & 1068.64 & 1419.47 & 3007.09 \\ 365.53 & 1099.56 & 1427.40 & 3670.24 \\ 629.71 & 1154.73 & 1680.87 & \\ 722.30 & 1241.66 & 2858.74 & \\ 839.97 & 1262.69 & 2896.35 & \end{array}$

1.30868500

$\begin{array}{cc}-0.23895500 & 0.00003100 \\ -0.62121500 & 0.92416000 \\ -0.62444100 & -0.92355900 \\ 0.57851100 & -0.00031200\end{array}$




$\begin{array}{rrrr}329 & \mathrm{H} & 0.04820600 \\ 330 & \mathrm{H} & -1.20353400 & -0.28 \\ 331 & \mathrm{C} & -2.09992600 & 0.37 \\ 332 & \mathrm{H} & -1.23025000 & -0.92 \\ 333 & \mathrm{H} & -1.23034700 & -0.928 \\ 334 & \mathrm{H} & & \\ 335 & \text { Frequencies[1/cm] } & & \\ & 45.22 & 1136.54 & 2900.66 \\ & 238.60 & 1244.93 & 2932.76 \\ & 316.43 & 1261.09 & 2970.39 \\ & 477.12 & 1326.73 & 2974.08 \\ & 709.85 & 1394.23 & 3003.13 \\ & 849.40 & 1413.18 & 3099.70 \\ & 864.12 & 1420.44 & \\ & 988.53 & 1431.84 & \\ & 1048.74 & 2896.94 & \end{array}$

336

337

338

339

340

341

342

343

344

345

346

347

348

349

350

351

352

353

354

355

356

357

358

359

360

361

362

363

364

365
TS-diss 1

$$
\text { C }
$$

$\mathrm{H}$

$\mathrm{H}$

C

$\mathrm{H}$

C

$\mathrm{H}$

C

$\mathrm{H}$

$\mathrm{H}$

C

$\mathrm{H}$

$\mathrm{H}$

C

$\mathrm{H}$

$\mathrm{H}$

$\mathrm{H}$

C

$\mathrm{H}$

$\mathrm{H}$

$\mathrm{H}$

$\mathrm{O}$

$\mathrm{H}$

Frequencies[1/cm]

$-573.95-247.08$

$58.79 \quad 282.18$

$78.04 \quad 340.49$

$102.43 \quad 363.70$

$$
\begin{array}{rrc}
-1.35591500 & -1.55763200 & 0.52839200 \\
-2.26119300 & -2.01302500 & 0.94879300 \\
-0.51518500 & -1.97414000 & 1.09070700 \\
-1.42192500 & -0.07158500 & 0.70114000 \\
-1.71366100 & 0.27807100 & 1.68685400 \\
-1.07069000 & 0.83173800 & -0.24897500 \\
-0.91512700 & 0.46112500 & -1.25644200 \\
1.13678600 & 1.06528500 & -0.13692800 \\
1.18438600 & 1.81158300 & 0.64846200 \\
1.27553400 & 1.46185800 & -1.13779700 \\
1.71691600 & -0.28042800 & 0.14906400 \\
1.29077100 & -1.01631000 & -0.53795400 \\
1.42913500 & -0.58816100 & 1.15671600 \\
3.24506800 & -0.29701400 & 0.03411000 \\
3.64509100 & -1.28804900 & 0.25062200 \\
3.55864300 & -0.01827800 & -0.97227800 \\
3.69479900 & 0.41145400 & 0.73045900 \\
-1.41457800 & 2.29260700 & -0.11974300 \\
-1.39182800 & 2.60324400 & 0.92495500 \\
-0.72338500 & 2.92295900 & -0.67715600 \\
-2.41894200 & 2.48294600 & -0.50291400 \\
-1.15890800 & -1.97566000 & -0.80733900 \\
-1.88174500 & -1.62781700 & -1.33666800
\end{array}
$$

$\begin{array}{lllll}793.20 & 1058.35 & 1303.35 & 1427.30 & 2953.42\end{array}$

$\begin{array}{llllll}838.57 & 1064.78 & 1330.07 & 1436.16 & 2956.14\end{array}$

$\begin{array}{llllll}867.05 & 1094.65 & 1337.59 & 1525.77 & 2965.13\end{array}$

$\begin{array}{llllll}870.92 & 1141.60 & 1345.66 & 2853.74 & 2967.96\end{array}$ 


$\begin{array}{lccccccc}366 & 125.66 & 373.26 & 930.62 & 1150.66 & 1394.18 & 2892.59 & 2975.57 \\ 367 & 162.66 & 536.97 & 932.19 & 1227.97 & 1412.51 & 2897.30 & 2997.26 \\ 368 & 186.46 & 614.73 & 961.76 & 1234.81 & 1414.65 & 2898.88 & 3006.46 \\ 369 & 207.19 & 677.11 & 990.47 & 1249.75 & 1421.69 & 2900.90 & 3042.53 \\ 370 & 243.97 & 727.84 & 1014.32 & 1271.72 & 1422.57 & 2930.07 & 3667.58 \\ 371 & & & & & & & \\ 372 & \text { P-diss2 } & & & & & & \\ 373 & \mathrm{C} & & 1.19294900 & -0.20676700 & 0.00004900 \\ 374 & \mathrm{H} & & 2.11892600 & 0.34521600 & 0.00006000 \\ 375 & \mathrm{H} & & 1.24250500 & -1.28831200 & 0.00052400 \\ 376 & \mathrm{C} & & 0.03764700 & 0.44009200 & -0.00023500 \\ 377 & \mathrm{H} & & -0.02402100 & 1.52021700 & -0.00023700 \\ 378 & \mathrm{O} & & -1.20018900 & -0.11313700 & 0.00032900 \\ 379 & \mathrm{H} & & -1.11947300 & -1.07197700 & -0.00185900\end{array}$

380 Frequencies[1/cm]

$\begin{array}{rr}436.61 & 1374.68 \\ 471.11 & 1646.11 \\ 687.44 & 3012.69 \\ 815.64 & 3050.17 \\ 918.57 & 3112.45 \\ 965.17 & 3661.27 \\ 1080.71 & \\ 1263.93 & \\ 1288.28 & \end{array}$

381

382

383

384

385

386

387

388

389

390

391

392

393

394

395

396

397

398

$\begin{array}{ccc}1.37936400 & 0.59914700 & -0.21622700 \\ 1.70184900 & 1.23636300 & -1.02840500 \\ -0.02468800 & 0.70057600 & 0.26619100 \\ -0.41875400 & 1.70326500 & 0.08576800 \\ -0.05396500 & 0.53940100 & 1.35068300 \\ -0.96932900 & -0.32330500 & -0.38656100 \\ -0.58791700 & -1.32936300 & -0.19778900 \\ -0.95099300 & -0.18179500 & -1.46953000 \\ -2.39512400 & -0.20267200 & 0.13437700 \\ -2.79741800 & 0.79170900 & -0.06481900 \\ -3.05688500 & -0.93162100 & -0.33230500 \\ -2.42776000 & -0.36177500 & 1.21324700 \\ 2.22046300 & -0.57127700 & 0.15102000 \\ 2.02997000 & -0.88063700 & 1.18167500 \\ 2.01383200 & -1.44521500 & -0.48109700 \\ 3.28393300 & -0.35514400 & 0.04977200\end{array}$

Frequencies[1/cm]

$\begin{array}{rrrrr}61.82 & 817.72 & 1199.53 & 1414.39 & 2915.95 \\ 95.57 & 829.27 & 1231.77 & 1419.11 & 2935.44 \\ 120.26 & 877.80 & 1258.44 & 1424.72 & 2960.45\end{array}$




$\begin{array}{lrrrr}226.52 & 938.27 & 1297.21 & 1432.87 & 2963.62 \\ 232.89 & 960.21 & 1315.62 & 2841.09 & 2970.20 \\ 309.63 & 1001.40 & 1338.30 & 2859.66 & 3028.60 \\ 395.38 & 1040.28 & 1343.70 & 2896.52 & 1414.65 \\ 463.21 & 1099.69 & 1398.26 & 2899.26 & 1421.69 \\ 707.20 & 1120.47 & 1408.69 & 2910.22 & 1422.57\end{array}$

399

400

401

402

403

404

405

406

407

408

409

410

411

412

413

414

415

416

417

418

419

420

421

422

423

424

425

426

427

428

429

430

431

432

433

434

435

436

437

438
TS-diss2$$
\text { C }
$$

$\mathrm{H}$

$\mathrm{H}$

C

$\mathrm{H}$

C

$\mathrm{H}$

C

$\mathrm{H}$

$\mathrm{H}$

C

$\mathrm{H}$

$\mathrm{H}$

C

$\mathrm{H}$

$\mathrm{H}$

$\mathrm{H}$

C

$\mathrm{H}$

$\mathrm{H}$

$\mathrm{H}$

$\mathrm{O}$

$\mathrm{H}$

Frequencies[1/cm]

$\begin{array}{ll}-618.80 & 260.35\end{array}$

$\begin{array}{ll}53.59 & 306.37\end{array}$

66.92

96.66

319.52

392.70

$105.83 \quad 434.61$

$195.25 \quad 475.85$

$205.23 \quad 553.40$

$214.97 \quad 688.84$

$235.55 \quad 710.33$

$$
\begin{array}{r}
-2.93138900 \\
-3.05263100 \\
-3.60367400 \\
-1.87207300 \\
-1.33100800 \\
-0.16791300 \\
-0.29289400 \\
0.97496600
\end{array}
$$

1.00583800

0.81227200

2.34055500

2.34336400

2.48964600

3.48048800

3.51173000

4.44671000

3.35096200

$-0.45241800$

$-0.44821300$

0.30234400

$-1.42034100$

$-1.74451100$

$-2.39131700$
$0.00922900 \quad-0.76300200$

$0.17412500 \quad-1.82179900$

$0.52028700-0.08591700$

$-0.70971600-0.30158900$

$-1.38541300-0.95086300$

$0.61836600-0.44303700$

$0.97072400-1.46292200$

$-0.32375100-0.22106400$

$-1.07178000-1.01925600$

$-0.86522700 \quad 0.71665200$

$0.37354500 \quad-0.15951000$

$1.08721800 \quad 0.66679900$

$0.95406200-1.07332500$

$-0.62152900 \quad 0.01736600$

$-1.32704500-0.81414000$

$-0.12054200 \quad 0.06867600$

$-1.19773300 \quad 0.93453700$

$1.62802500 \quad 0.62026900$

$1.15497200 \quad 1.60513200$

$2.42094200 \quad 0.63932700$

$2.10569600 \quad 0.46137500$

$-1.08323500 \quad 1.01303900$

$-0.59941400 \quad 1.53481600$

$\begin{array}{lllll}753.02 & 1055.88 & 1302.30 & 1425.27 & 2934.99\end{array}$

$\begin{array}{llllll}818.54 & 1058.42 & 1313.32 & 1433.24 & 2956.04\end{array}$

$\begin{array}{lllll}835.82 & 1101.55 & 1337.19 & 1492.64 & 2964.90\end{array}$

$\begin{array}{llllll}867.62 & 1131.48 & 1339.19 & 2871.85 & 2970.75\end{array}$

$\begin{array}{llllll}895.15 & 1203.53 & 1363.46 & 2873.33 & 2980.86\end{array}$

$\begin{array}{llllll}914.91 & 1216.84 & 1404.14 & 2896.34 & 3006.99\end{array}$

$\begin{array}{llllll}960.48 & 1238.53 & 1414.64 & 2899.72 & 3027.36\end{array}$

$\begin{array}{llllll}986.17 & 1257.87 & 1416.33 & 2909.48 & 3107.37\end{array}$

$\begin{array}{llllll}1007.67 & 1265.52 & 1419.55 & 2922.61 & 3668.07\end{array}$

P-add2

C

$-2.82474900$

$0.14056600 \quad-0.76191100$

$\mathrm{H}$

$-3.03755500$

$\begin{array}{ll}0.32560900 & -1.80404400\end{array}$

$\mathrm{H}$

$-3.46029700$

$0.58544200 \quad-0.01052500$ 


\begin{tabular}{|c|c|c|c|c|c|}
\hline 439 & $\mathrm{C}$ & & -1.56334700 & -0.53069300 & -0.35659900 \\
\hline 440 & $\mathrm{H}$ & & -1.33720500 & -1.32409500 & -1.08334700 \\
\hline 441 & $\mathrm{C}$ & & -0.34250000 & 0.40989000 & -0.33792600 \\
\hline 442 & $\mathrm{H}$ & & -0.27731900 & 0.84247900 & -1.34208400 \\
\hline 443 & $\mathrm{C}$ & & 0.92963300 & -0.39893400 & -0.08024700 \\
\hline 444 & $\mathrm{H}$ & & 0.97955500 & -1.21450700 & -0.80939000 \\
\hline 445 & $\mathrm{H}$ & & 0.85474600 & -0.86926200 & 0.90332400 \\
\hline 446 & $\mathrm{C}$ & & 2.21909800 & 0.41032600 & -0.16576600 \\
\hline 447 & $\mathrm{H}$ & & 2.24427400 & 1.15383100 & 0.63257600 \\
\hline 448 & $\mathrm{H}$ & & 2.23748100 & 0.96597900 & -1.10802300 \\
\hline 449 & $\mathrm{C}$ & & 3.45560300 & -0.47479700 & -0.06591500 \\
\hline 450 & $\mathrm{H}$ & & 3.47963100 & -1.20199500 & -0.87879800 \\
\hline 451 & $\mathrm{H}$ & & 4.37407700 & 0.10961000 & -0.11104300 \\
\hline 452 & $\mathrm{H}$ & & 3.45677600 & -1.03015700 & 0.87314400 \\
\hline 453 & $\mathrm{C}$ & & -0.52871100 & 1.53863600 & 0.67172900 \\
\hline 454 & $\mathrm{H}$ & & -0.56311700 & 1.13641100 & 1.68430700 \\
\hline 455 & $\mathrm{H}$ & & 0.28888000 & 2.25520100 & 0.61223300 \\
\hline 456 & $\mathrm{H}$ & & -1.45663700 & 2.08063600 & 0.48866300 \\
\hline 457 & $\mathrm{O}$ & & -1.67386900 & -1.09313000 & 0.94523800 \\
\hline 458 & $\mathrm{H}$ & & -2.46249600 & -1.64010400 & 0.97091500 \\
\hline 459 & \multicolumn{2}{|c|}{ Frequencies $[1 / \mathrm{cm}]$} & & & \\
\hline 460 & 49.34 & 292.32 & $823.59 \quad 1094.05$ & $1309.95 \quad 1425.67$ & $7 \quad 2922.26$ \\
\hline 461 & 75.44 & 296.16 & $842.45 \quad 1115.53$ & $1316.35 \quad 1429.13$ & $3 \quad 2938.79$ \\
\hline 462 & 95.99 & 335.86 & $879.83 \quad 1130.18$ & $1328.13 \quad 1434.44$ & $4 \quad 2963.69$ \\
\hline 463 & 127.49 & 423.85 & $912.64 \quad 1177.03$ & $1338.87 \quad 2826.60$ & $0 \quad 2970.07$ \\
\hline 464 & 140.78 & 459.37 & $953.84 \quad 1200.93$ & $1344.60 \quad 2878.16$ & $6 \quad 2973.21$ \\
\hline 465 & 207.68 & 512.32 & $988.04 \quad 1225.78$ & $1378.24 \quad 2880.45$ & $5 \quad 2982.90$ \\
\hline 466 & 236.42 & 602.61 & $1009.95 \quad 1258.90$ & $1408.56 \quad 2888.17$ & $7 \quad 3005.24$ \\
\hline 467 & 270.68 & 708.16 & $1041.46 \quad 1266.76$ & $1420.12 \quad 2898.62$ & 23109.63 \\
\hline 468 & 279.75 & 779.62 & $1048.32 \quad 1294.24$ & $1424.22 \quad 2908.84$ & $4 \quad 3683.55$ \\
\hline \multicolumn{6}{|l|}{469} \\
\hline 470 & \multicolumn{5}{|l|}{ TS-add2 } \\
\hline 471 & $\mathrm{C}$ & & -2.80983300 & 0.16654700 & -0.50461000 \\
\hline 472 & $\mathrm{H}$ & & -3.62981000 & -0.37512400 & -0.95652700 \\
\hline 473 & $\mathrm{H}$ & & -3.05882300 & 0.93942500 & 0.20816700 \\
\hline 474 & $\mathrm{C}$ & & -1.53135600 & -0.14948300 & -0.79482900 \\
\hline 475 & $\mathrm{H}$ & & -1.35144200 & -0.91590500 & -1.54098500 \\
\hline 476 & $\mathrm{C}$ & & -0.31209500 & 0.62336600 & -0.37142000 \\
\hline 477 & $\mathrm{H}$ & & -0.06683400 & 1.25781100 & -1.23420400 \\
\hline 478 & $\mathrm{C}$ & & 0.88807900 & -0.30550200 & -0.15137100 \\
\hline 479 & $\mathrm{H}$ & & 0.88708800 & -1.07755900 & -0.92680800 \\
\hline 480 & $\mathrm{H}$ & & 0.75770500 & -0.82989900 & 0.79660900 \\
\hline 481 & $\mathrm{C}$ & & 2.23435200 & 0.40908300 & -0.17683400 \\
\hline 482 & $\mathrm{H}$ & & 2.27245600 & 1.16707300 & 0.60787900 \\
\hline 483 & $\mathrm{H}$ & & 2.34448100 & 0.94121400 & -1.12595400 \\
\hline 484 & $\mathrm{C}$ & & 3.39627700 & $\begin{array}{c}-0.55983300 \\
\text { S-13 }\end{array}$ & 0.00561900 \\
\hline
\end{tabular}




\begin{tabular}{|c|c|c|c|c|c|c|}
\hline 485 & \multicolumn{2}{|l|}{$\mathrm{H}$} & \multicolumn{2}{|c|}{3.40261800} & -1.31109800 & -0.78543500 \\
\hline 486 & \multicolumn{2}{|l|}{$\mathrm{H}$} & \multicolumn{2}{|c|}{4.35638500} & -0.04509700 & -0.01269400 \\
\hline 487 & \multicolumn{2}{|l|}{$\mathrm{H}$} & \multicolumn{2}{|c|}{3.31445900} & -1.08529900 & 0.95796900 \\
\hline 488 & \multicolumn{2}{|l|}{$\mathrm{C}$} & \multicolumn{2}{|c|}{-0.57659900} & 1.52217500 & 0.83078900 \\
\hline 489 & \multicolumn{2}{|l|}{$\mathrm{H}$} & \multicolumn{2}{|c|}{-0.93011900} & 0.91758800 & 1.66680600 \\
\hline 490 & \multicolumn{2}{|l|}{$\mathrm{H}$} & \multicolumn{2}{|c|}{0.33147000} & 2.03998800 & 1.13378300 \\
\hline 491 & \multicolumn{2}{|l|}{$\mathrm{H}$} & \multicolumn{2}{|c|}{-1.32529000} & 2.28260500 & 0.60941600 \\
\hline 492 & \multicolumn{2}{|l|}{$\mathrm{O}$} & \multicolumn{2}{|c|}{-1.57579100} & -1.52343200 & 0.85718500 \\
\hline 493 & $\mathrm{H}$ & & -2.43096 & -1.9 & 5638600 & 2043000 \\
\hline 494 & Frequencies[1 & $/ \mathrm{cm}]$ & & & & \\
\hline 495 & -309.86 & 251.60 & 790.40 & 1024.41 & $1299.74 \quad 1432.5$ & $\begin{array}{ll}51 & 2949.14\end{array}$ \\
\hline 496 & 39.48 & 310.86 & 824.50 & 1069.32 & $\begin{array}{ll}1328.50 & 1439.7\end{array}$ & $73 \quad 2964.74$ \\
\hline 497 & 74.65 & 316.65 & 860.98 & 1119.47 & $\begin{array}{ll}1336.75 & 1545.1\end{array}$ & $16 \quad 2970.88$ \\
\hline 498 & 94.08 & 373.56 & 880.54 & 1142.61 & $1341.54 \quad 2846.7$ & $\begin{array}{ll}77 \quad 2972.98\end{array}$ \\
\hline 499 & 109.56 & 424.96 & 907.38 & 1196.51 & $1373.08 \quad 2887.0$ & $07 \quad 2985.91$ \\
\hline 500 & 134.57 & 524.15 & 955.05 & 1222.65 & $\begin{array}{ll}1407.35 \quad 2891.7\end{array}$ & $\begin{array}{ll}78 \quad 3004.77\end{array}$ \\
\hline 501 & 204.28 & 595.92 & 963.39 & 1249.02 & $\begin{array}{ll}1418.81 \quad 2899.4\end{array}$ & $42 \quad 3013.17$ \\
\hline 502 & 232.20 & 675.26 & 982.61 & 1260.31 & $1424.49 \quad 2907.8$ & 393096.90 \\
\hline 503 & 241.10 & 703.41 & 1021.43 & 1278.01 & $1426.05 \quad 2925.6$ & $\begin{array}{ll}65 & 3610.37\end{array}$ \\
\hline 504 & & & & & & \\
\hline 505 & P-abs 1 & & & & & \\
\hline 506 & $\mathrm{C}$ & & 1.84 & 683200 & 1.87290700 & -0.19696700 \\
\hline 507 & $\mathrm{H}$ & & & 7527400 & 2.81417400 & 0.10528400 \\
\hline 508 & $\mathrm{C}$ & & & 0604500 & 0.79460600 & 0.38840400 \\
\hline 509 & $\mathrm{H}$ & & & 3363800 & 0.73041600 & 1.48116700 \\
\hline 510 & $\mathrm{C}$ & & & 5078400 & -0.42202500 & -0.30940400 \\
\hline 511 & $\mathrm{H}$ & & & 6900900 & -0.22959200 & -1.38503100 \\
\hline 512 & $\mathrm{C}$ & & -0.5 & 834100 & -0.67712700 & 0.11566200 \\
\hline 513 & $\mathrm{H}$ & & -0.6 & 2692300 & -0.83066300 & 1.20053700 \\
\hline 514 & $\mathrm{H}$ & & -0.9 & 156300 & -1.61640300 & -0.33583200 \\
\hline 515 & $\mathrm{C}$ & & -1.5 & 479600 & 0.43605300 & -0.26720900 \\
\hline 516 & $\mathrm{H}$ & & -1.5 & 635600 & 0.60251200 & -1.34622000 \\
\hline 517 & $\mathrm{H}$ & & -1.2 & 1804700 & 1.36944000 & 0.20185300 \\
\hline 518 & $\mathrm{C}$ & & -3.0 & 015800 & 0.11613000 & 0.13017900 \\
\hline 519 & $\mathrm{H}$ & & -3.6 & 3183400 & 0.91926300 & -0.14757500 \\
\hline 520 & $\mathrm{H}$ & & -3.3 & 4462200 & -0.79849900 & -0.35484800 \\
\hline 521 & $\mathrm{H}$ & & -3.0 & 999400 & -0.03242100 & 1.20819800 \\
\hline 522 & $\mathrm{C}$ & & & 2718600 & -1.63648900 & -0.00430400 \\
\hline 523 & $\mathrm{H}$ & & & 3913700 & -1.83650300 & 1.06931200 \\
\hline 524 & $\mathrm{H}$ & & & 4298900 & -2.52471400 & -0.50681400 \\
\hline 525 & $\mathrm{H}$ & & & 5396200 & -1.47135000 & -0.32819000 \\
\hline 526 & Frequencies[] & $/ \mathrm{cm}]$ & & & & \\
\hline 527 & 67.12 & 408.27 & 924.49 & 1228.33 & $1420.68 \quad 2899.1$ & \\
\hline 528 & 78.33 & 483.19 & 973.94 & 1261.32 & $1424.28 \quad 2901.3$ & \\
\hline 529 & 111.76 & 661.47 & 1005.08 & 1281.36 & $1425.98 \quad 2912.2$ & \\
\hline 530 & 170.41 & 704.16 & 1025.62 & 1290.56 & $\begin{array}{c}1434.522935 .4 \\
\text { S-14 }\end{array}$ & \\
\hline
\end{tabular}




\begin{tabular}{|c|c|c|c|c|c|c|c|}
\hline 531 & 226.08 & 731.91 & 1078.62 & 1325.57 & \multicolumn{3}{|c|}{$1614.74 \quad 2960.05$} \\
\hline 532 & 236.00 & 822.32 & 1106.61 & 1336.30 & \multicolumn{3}{|c|}{$2869.94 \quad 2962.77$} \\
\hline 533 & 290.46 & 841.33 & 1122.37 & 1340.79 & \multicolumn{3}{|c|}{$2888.84 \quad 2971.04$} \\
\hline 534 & 302.77 & 843.08 & 1171.22 & 1406.77 & \multicolumn{3}{|c|}{$2893.86 \quad 2976.35$} \\
\hline 535 & 339.73 & 875.17 & 1207.34 & 1418.87 & \multicolumn{3}{|c|}{$2895.57 \quad 3096.37$} \\
\hline 36 & & & & & & & \\
\hline 537 & \multicolumn{7}{|l|}{ TS-abs 1} \\
\hline 538 & \multicolumn{2}{|l|}{$\mathrm{C}$} & \multicolumn{2}{|c|}{-1.78039300} & \multicolumn{2}{|c|}{-0.67686700} & 1.15523200 \\
\hline 539 & \multicolumn{2}{|l|}{$\mathrm{H}$} & \multicolumn{2}{|c|}{-2.27166600} & \multicolumn{2}{|c|}{-1.03757500} & 2.04899600 \\
\hline 540 & \multicolumn{2}{|l|}{$\mathrm{H}$} & \multicolumn{2}{|c|}{-2.03469200} & \multicolumn{2}{|c|}{-1.30876600} & 0.16754400 \\
\hline 541 & \multicolumn{2}{|l|}{$\mathrm{C}$} & \multicolumn{2}{|c|}{-0.94979400} & \multicolumn{2}{|c|}{0.33878700} & 1.05321000 \\
\hline 542 & \multicolumn{2}{|l|}{$\mathrm{H}$} & \multicolumn{2}{|c|}{-0.68331800} & \multicolumn{2}{|c|}{0.88435900} & 1.96043500 \\
\hline 543 & \multicolumn{2}{|l|}{$\mathrm{C}$} & -0.30 & 446600 & 0.8306 & 7100 & -0.21063600 \\
\hline 544 & $\mathrm{H}$ & & -0.67 & 737500 & 0.2188 & 4100 & -1.03528700 \\
\hline 545 & $\mathrm{C}$ & & 1.21 & 810900 & 0.6712 & 1800 & -0.13874000 \\
\hline 546 & $\mathrm{H}$ & & $1.5 \mathrm{C}$ & 989100 & 1.2528 & 37200 & 0.70814300 \\
\hline 547 & $\mathrm{H}$ & & 1.65 & 197700 & 1.1182 & 29600 & -1.03810500 \\
\hline 548 & $\mathrm{C}$ & & 1.68 & 356800 & -0.7744 & 4200 & -0.02051900 \\
\hline 549 & $\mathrm{H}$ & & 1.25 & 5051000 & -1.3554 & 8100 & -0.83904500 \\
\hline 550 & $\mathrm{H}$ & & 1.25 & 087400 & -1.2094 & 8400 & 0.90045300 \\
\hline 551 & $\mathrm{C}$ & & 3.20 & 191000 & -0.8944 & 6700 & -0.04254700 \\
\hline 552 & $\mathrm{H}$ & & 3.52 & 289600 & -1.9314 & 7400 & 0.04857000 \\
\hline 553 & $\mathrm{H}$ & & 3.61 & 104700 & -0.4977 & 5700 & -0.97291600 \\
\hline 554 & $\mathrm{H}$ & & 3.64 & 4784100 & -0.3326 & 6400 & 0.77982000 \\
\hline 555 & $\mathrm{C}$ & & -0.68 & 376100 & $2.2918^{\prime}$ & 7200 & -0.45271400 \\
\hline 556 & $\mathrm{H}$ & & -0.32 & 887300 & 2.9204 & 8800 & 0.36680900 \\
\hline 557 & $\mathrm{H}$ & & -0.23 & 141600 & 2.6567 & 3400 & -1.37509000 \\
\hline 558 & $\mathrm{H}$ & & -1.76 & 410900 & 2.4137 & 7000 & -0.52678500 \\
\hline 559 & $\mathrm{O}$ & & -2.46 & 855800 & -1.68836 & 6400 & -1.01456400 \\
\hline 560 & $\mathrm{H}$ & & -3.14 & 615900 & -1.00587 & 7000 & -1.13674800 \\
\hline 561 & Frequencies[1 & /cm] & & & & & \\
\hline 562 & -1078.73 & 235.76 & 772.18 & 1027.83 & 1266.77 & 1424.78 & $8 \quad 2919.17$ \\
\hline 563 & 19.77 & 294.58 & 828.30 & 1079.31 & 1291.96 & 1426.53 & $3 \quad 2935.50$ \\
\hline 564 & 62.71 & 321.25 & 839.80 & 1117.22 & 1321.14 & 1434.40 & $0 \quad 2939.81$ \\
\hline 565 & 95.30 & 342.65 & 865.91 & 1132.42 & 1327.30 & 1628.24 & $4 \quad 2961.23$ \\
\hline 566 & 105.84 & 402.17 & 909.09 & 1167.38 & 1337.92 & 2870.44 & $4 \quad 2962.43$ \\
\hline 567 & 147.79 & 490.32 & 917.52 & 1192.49 & 1340.99 & 2894.37 & $7 \quad 2970.80$ \\
\hline 568 & 161.56 & 579.10 & 936.22 & 1200.37 & 1407.51 & 2896.07 & $7 \quad 2973.33$ \\
\hline 569 & 181.06 & 695.74 & 983.83 & 1231.97 & 1418.81 & 2899.76 & $6 \quad 3050.79$ \\
\hline 570 & 225.18 & 715.10 & 1006.71 & 1245.90 & 1421.82 & 2905.53 & $3 \quad 3603.54$ \\
\hline 571 & & & & & & & \\
\hline 572 & P-abs2 & & & & & & \\
\hline 573 & $\mathrm{C}$ & & -1.943 & 68900 & 1.84657 & 900 & -0.02328500 \\
\hline 574 & $\mathrm{H}$ & & -2.30 & 740200 & 2.5528 & 3200 & -0.76036800 \\
\hline 575 & $\mathrm{H}$ & & -2.04 & 901000 & 2.1327 & 8200 & 1.02372000 \\
\hline 576 & $\mathrm{C}$ & & -1.40 & 317900 & $\begin{array}{r}0.7066 \\
\text { S-1 }\end{array}$ & $\begin{array}{l}0900 \\
15\end{array}$ & -0.35715200 \\
\hline
\end{tabular}




\begin{tabular}{|c|c|c|c|c|c|}
\hline 577 & $\mathrm{C}$ & & -0.82225800 & -0.48710500 & 0.28436400 \\
\hline 578 & $\mathrm{H}$ & & -0.84849400 & -0.31580200 & 1.37120800 \\
\hline 579 & $\mathrm{C}$ & & 0.64074000 & -0.69028200 & -0.12909800 \\
\hline 580 & $\mathrm{H}$ & & 0.67839100 & -0.84228500 & -1.21230400 \\
\hline 581 & $\mathrm{H}$ & & 0.99974400 & -1.61555400 & 0.33055400 \\
\hline 582 & $\mathrm{C}$ & & 1.55930300 & 0.46006300 & 0.25893900 \\
\hline 583 & $\mathrm{H}$ & & 1.49070600 & 0.62409900 & 1.33801600 \\
\hline 584 & $\mathrm{H}$ & & 1.20637000 & 1.37823800 & -0.21444100 \\
\hline 585 & $\mathrm{C}$ & & 3.00782000 & 0.19731700 & -0.13231600 \\
\hline 586 & $\mathrm{H}$ & & 3.65536400 & 1.02719600 & 0.14827600 \\
\hline 587 & $\mathrm{H}$ & & 3.38672900 & -0.70281400 & 0.35414100 \\
\hline 588 & $\mathrm{H}$ & & 3.09726500 & 0.05274800 & -1.20995300 \\
\hline 589 & $\mathrm{C}$ & & -1.65850100 & -1.72864500 & -0.03593300 \\
\hline 590 & $\mathrm{H}$ & & -1.64919000 & -1.91973300 & -1.10969400 \\
\hline 591 & $\mathrm{H}$ & & -1.24865000 & -2.60207700 & 0.47147200 \\
\hline 592 & $\mathrm{H}$ & & -2.69324300 & -1.59685300 & 0.27626400 \\
\hline 593 & \multicolumn{2}{|c|}{ Frequencies $[1 / \mathrm{cm}]$} & & & \\
\hline 594 & 67.87 & 386.00 & $930.71 \quad 1249.44$ & \multicolumn{2}{|c|}{$1419.23 \quad 2902.07$} \\
\hline 595 & 77.07 & 464.03 & $971.12 \quad 1267.47$ & \multicolumn{2}{|c|}{$1424.79 \quad 2915.77$} \\
\hline 596 & 110.63 & 593.21 & $1006.56 \quad 1273.17$ & \multicolumn{2}{|c|}{$1425.97 \quad 2922.41$} \\
\hline 597 & 170.50 & 705.75 & $1026.03 \quad 1324.02$ & \multicolumn{2}{|c|}{$1434.14 \quad 2936.57$} \\
\hline 598 & 207.50 & 757.22 & $1075.84 \quad 1336.27$ & \multicolumn{2}{|c|}{$1671.77 \quad 2963.61$} \\
\hline 599 & 233.67 & 832.29 & $1099.90 \quad 1340.72$ & \multicolumn{2}{|c|}{$2820.97 \quad 2969.88$} \\
\hline 600 & 254.91 & 858.47 & $1122.54 \quad 1348.17$ & \multicolumn{2}{|c|}{$2881.00 \quad 2971.35$} \\
\hline 601 & 284.96 & 863.28 & $1188.10 \quad 1405.55$ & \multicolumn{2}{|c|}{$2893.86 \quad 2980.96$} \\
\hline 602 & 337.88 & 903.32 & $1220.03 \quad 1418.15$ & \multicolumn{2}{|c|}{$2899.30 \quad 3026.73$} \\
\hline \multicolumn{6}{|l|}{603} \\
\hline 604 & abs2 & & & & \\
\hline 605 & $\mathrm{C}$ & & 1.77060900 & -0.81373300 & 1.60754500 \\
\hline 606 & $\mathrm{H}$ & & 2.28551100 & -1.76494200 & 1.61970400 \\
\hline 607 & $\mathrm{H}$ & & 1.67898100 & -0.28139200 & 2.54921600 \\
\hline 608 & $\mathrm{C}$ & & 1.28210200 & -0.30394800 & 0.49523400 \\
\hline 609 & $\mathrm{H}$ & & 1.43766600 & -0.92146900 & -0.49420500 \\
\hline 610 & $\mathrm{C}$ & & 0.54157600 & 0.97114700 & 0.25729400 \\
\hline 611 & $\mathrm{H}$ & & 0.42635300 & 1.46578400 & 1.22894000 \\
\hline 612 & $\mathrm{C}$ & & -0.85108100 & 0.69863400 & -0.32212800 \\
\hline 613 & $\mathrm{H}$ & & -0.72979600 & 0.20668600 & -1.29239500 \\
\hline 614 & $\mathrm{H}$ & & -1.33578300 & 1.65955000 & -0.51644900 \\
\hline 615 & $\mathrm{C}$ & & -1.74715100 & -0.14649900 & 0.57352800 \\
\hline 616 & $\mathrm{H}$ & & -1.82118500 & 0.32486400 & 1.55756500 \\
\hline 617 & $\mathrm{H}$ & & -1.28139300 & -1.12053800 & 0.74152500 \\
\hline 618 & $\mathrm{C}$ & & -3.13872400 & -0.33408600 & -0.01709600 \\
\hline 619 & $\mathrm{H}$ & & -3.76887600 & -0.94770500 & 0.62568500 \\
\hline 620 & $\mathrm{H}$ & & -3.63562500 & 0.62722300 & -0.15486500 \\
\hline 621 & $\mathrm{H}$ & & -3.08289800 & -0.81793900 & -0.99346200 \\
\hline 622 & $\mathrm{C}$ & & 1.34476700 & $\begin{array}{c}1.87398000 \\
\text { S-16 }\end{array}$ & -0.67994800 \\
\hline
\end{tabular}




\begin{tabular}{|c|c|c|c|c|c|c|}
\hline 623 & \multicolumn{2}{|l|}{$\mathrm{H}$} & \multicolumn{2}{|c|}{1.49264400} & 1.37534500 & -1.63942400 \\
\hline 624 & \multicolumn{2}{|l|}{$\mathrm{H}$} & \multicolumn{2}{|c|}{0.81288500} & 2.80852400 & -0.85955500 \\
\hline 625 & \multicolumn{2}{|l|}{$\mathrm{H}$} & \multicolumn{2}{|c|}{2.32363200} & 2.10508900 & -0.26217900 \\
\hline 626 & \multicolumn{2}{|l|}{$\mathrm{O}$} & \multicolumn{2}{|c|}{1.19024200} & -1.76583400 & -1.55566300 \\
\hline 627 & \multicolumn{2}{|l|}{$\mathrm{H}$} & \multicolumn{2}{|c|}{0.46335400} & -2.26538300 & -1.15137100 \\
\hline 628 & \multicolumn{2}{|c|}{ Frequencies $[1 / \mathrm{cm}]$} & & & & \\
\hline 629 & -756.49 & 238.29 & 769.88 & 1026.30 & $1278.49 \quad 1426.71$ & $\begin{array}{ll}71 & 2913.25\end{array}$ \\
\hline 630 & 31.99 & 269.00 & 833.15 & 1076.62 & $1323.76 \quad 1428$ & $.01 \quad 2932.83$ \\
\hline 631 & 68.66 & 313.71 & 850.86 & 1117.64 & \multicolumn{2}{|c|}{$\begin{array}{lll}1334.95 & 1434.86 & 2963.00\end{array}$} \\
\hline 632 & 71.09 & 341.55 & 870.37 & 1138.37 & \multicolumn{2}{|c|}{$\begin{array}{lll}1342.53 & 1647.73 & 2967.86\end{array}$} \\
\hline 633 & 100.37 & 396.56 & 907.43 & 1164.33 & \multicolumn{2}{|c|}{$\begin{array}{lll}1355.44 & 2869.88 & 2970.87\end{array}$} \\
\hline 634 & 139.83 & 493.85 & 917.17 & 1195.65 & \multicolumn{2}{|c|}{$\begin{array}{lll}1360.42 & 2883.02 \quad 2978.00\end{array}$} \\
\hline 635 & 150.31 & 635.15 & 940.57 & 1224.29 & \multicolumn{2}{|c|}{$\begin{array}{lll}1410.77 & 2893.02 & 2978.84\end{array}$} \\
\hline 636 & 175.60 & 693.48 & 983.50 & 1250.39 & \multicolumn{2}{|c|}{$\begin{array}{lll}1418.78 & 2899.15 & 3068.83\end{array}$} \\
\hline 637 & \multirow[t]{2}{*}{226.52} & 710.36 & \multirow{2}{*}{\multicolumn{2}{|c|}{$1006.65 \quad 12$}} & \multicolumn{2}{|c|}{$\begin{array}{lll}1421.85 & 2899.87 & 3595.81\end{array}$} \\
\hline \multicolumn{5}{|l|}{638} & & \\
\hline 639 & P-abs3 & & & & & \\
\hline 640 & $\mathrm{C}$ & & -2.59 & 160300 & -1.18936300 & -0.00016800 \\
\hline 641 & $\mathrm{H}$ & & -2.9 & 4355700 & -2.21073300 & -0.00009700 \\
\hline 642 & $\mathrm{H}$ & & -3.3 & 4503300 & -0.41172400 & -0.00062500 \\
\hline 643 & $\mathrm{C}$ & & -1.2 & 100200 & -0.90229000 & 0.00020400 \\
\hline 644 & $\mathrm{H}$ & & -0.5 & 5519300 & -1.74116600 & 0.00052900 \\
\hline 645 & $\mathrm{C}$ & & -0.7 & 705500 & 0.37599700 & 0.00017100 \\
\hline 646 & $\mathrm{C}$ & & 0.7 & 6791500 & 0.64001100 & 0.00007900 \\
\hline 647 & $\mathrm{H}$ & & 1.0 & 0496300 & 1.27008800 & 0.86790400 \\
\hline 648 & $\mathrm{H}$ & & 1.0 & 0486000 & 1.27009600 & -0.86777800 \\
\hline 649 & $\mathrm{C}$ & & 1.6 & 9148800 & -0.56963400 & -0.00000900 \\
\hline 650 & $\mathrm{H}$ & & 1.4 & 8343100 & -1.18819900 & -0.87567100 \\
\hline 651 & $\mathrm{H}$ & & 1.4 & 8356500 & -1.18827900 & 0.87562200 \\
\hline 652 & $\mathrm{C}$ & & 3.1 & 5823500 & -0.15740200 & -0.00011600 \\
\hline 653 & $\mathrm{H}$ & & 3.8 & 1686600 & -1.02491200 & 0.00023500 \\
\hline 654 & $\mathrm{H}$ & & 3.3 & 9379300 & 0.44170900 & -0.88089100 \\
\hline 655 & $\mathrm{H}$ & & 3.3 & 9373100 & 0.44237700 & 0.88022300 \\
\hline 656 & $\mathrm{C}$ & & -1.5 & 932700 & 1.57933200 & -0.00003100 \\
\hline 657 & $\mathrm{H}$ & & -2.2 & 5152200 & 1.58987600 & 0.87715600 \\
\hline 658 & $\mathrm{H}$ & & -1.0 & 1895500 & 2.50073200 & 0.00034000 \\
\hline 659 & $\mathrm{H}$ & & -2.2 & 5085600 & 1.59023500 & -0.87772700 \\
\hline 660 & Frequencies & $/ \mathrm{cm}]$ & & & & \\
\hline 661 & 70.02 & 390.82 & 943.95 & 1242.67 & $1420.36 \quad 2901$ & \\
\hline 662 & 101.30 & 554.85 & 957.57 & 1257.56 & $1424.26 \quad 2927$ & \\
\hline 663 & 109.95 & 557.14 & 989.80 & 1291.01 & 1426.542933 & \\
\hline 664 & 159.24 & 706.14 & 1014.55 & 1333.57 & 1435.142964 & \\
\hline 665 & 208.65 & 747.13 & 1057.07 & 1342.06 & 1449.552972 & \\
\hline 666 & 236.59 & 767.92 & 1068.52 & 1349.71 & $2842.81 \quad 2973$ & \\
\hline 667 & 268.80 & 819.27 & 1134.96 & 1374.18 & 2859.613015 & \\
\hline 668 & 342.25 & 878.79 & 1177.98 & 1399.93 & $\begin{array}{c}2881.85 \quad 3023 \\
\text { S-17 }\end{array}$ & \\
\hline
\end{tabular}




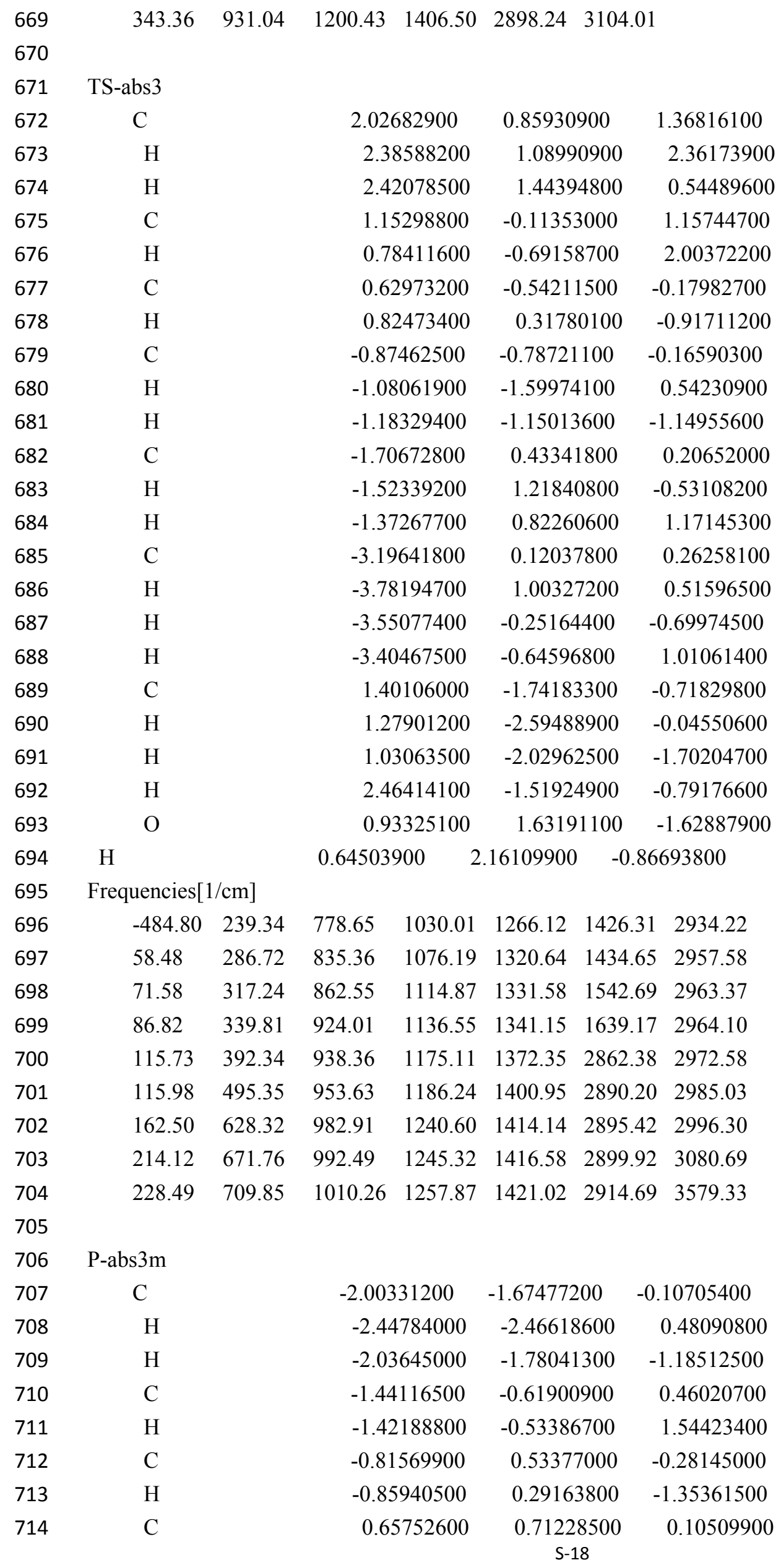




\begin{tabular}{|c|c|c|c|c|c|}
\hline 715 & $\mathrm{H}$ & & 0.71625200 & 0.90874800 & 1.18097200 \\
\hline 716 & $\mathrm{H}$ & & 1.03963700 & 1.60755900 & -0.39392700 \\
\hline 717 & $\mathrm{C}$ & & 1.53730000 & -0.47891000 & -0.24823500 \\
\hline 718 & $\mathrm{H}$ & & 1.44789400 & -0.68328700 & -1.31881300 \\
\hline 719 & $\mathrm{H}$ & & 1.16652700 & -1.36911100 & 0.26343300 \\
\hline 720 & $\mathrm{C}$ & & 2.99833200 & -0.24330800 & 0.11262400 \\
\hline 721 & $\mathrm{H}$ & & 3.61804500 & -1.10198000 & -0.14316600 \\
\hline 722 & $\mathrm{H}$ & & 3.39521800 & 0.62556100 & -0.41456500 \\
\hline 723 & $\mathrm{H}$ & & 3.10805600 & -0.05843900 & 1.18229100 \\
\hline 724 & $\mathrm{C}$ & & -1.59367700 & 1.78100500 & -0.02645200 \\
\hline 725 & $\mathrm{H}$ & & -1.09332800 & 2.71241300 & 0.19555800 \\
\hline 726 & $\mathrm{H}$ & & -2.66855200 & 1.78101000 & -0.12661900 \\
\hline 727 & \multicolumn{2}{|c|}{ Frequencies $[1 / \mathrm{cm}]$} & & & \\
\hline 728 & 70.13 & 375.80 & $915.08 \quad 1213.59$ & \multicolumn{2}{|c|}{$1412.98 \quad 2915.47$} \\
\hline 729 & 87.43 & 494.68 & $965.01 \quad 1247.81$ & \multicolumn{2}{|c|}{$1420.18 \quad 2934.67$} \\
\hline 730 & 112.68 & 513.54 & $987.30 \quad 1251.68$ & \multicolumn{2}{|c|}{$1425.66 \quad 2963.15$} \\
\hline 731 & 160.40 & 667.94 & $1009.31 \quad 1265.38$ & \multicolumn{2}{|c|}{$\begin{array}{ll}1434.41 \quad 2971.06\end{array}$} \\
\hline 732 & 176.95 & 710.34 & $1032.17 \quad 1290.10$ & \multicolumn{2}{|c|}{$1634.23 \quad 2985.48$} \\
\hline 733 & 234.84 & 820.34 & $1057.54 \quad 1325.05$ & \multicolumn{2}{|c|}{$2874.63 \quad 2993.92$} \\
\hline 734 & 276.06 & 831.84 & $1104.76 \quad 1340.60$ & \multicolumn{2}{|c|}{$2888.36 \quad 2998.04$} \\
\hline 735 & 318.99 & 868.30 & $1131.88 \quad 1377.22$ & \multicolumn{2}{|c|}{$2895.28 \quad 3077.84$} \\
\hline 736 & 332.19 & 902.33 & $1193.48 \quad 1383.85$ & \multicolumn{2}{|c|}{$2899.54 \quad 3097.32$} \\
\hline \multicolumn{6}{|l|}{737} \\
\hline 738 & abs $3 m$ & & & & \\
\hline 739 & $\mathrm{C}$ & & -0.97048400 & 2.47176700 & -0.36980300 \\
\hline 740 & $\mathrm{H}$ & & -1.29824000 & 3.04460700 & -1.22669000 \\
\hline 741 & $\mathrm{H}$ & & -0.73359900 & 3.02422900 & 0.53209100 \\
\hline 742 & $\mathrm{C}$ & & -0.85768200 & 1.15362000 & -0.41679100 \\
\hline 743 & $\mathrm{H}$ & & -1.09354100 & 0.62091100 & -1.33548800 \\
\hline 744 & $\mathrm{C}$ & & -0.42019500 & 0.27974900 & 0.72303700 \\
\hline 745 & $\mathrm{H}$ & & -0.14479300 & 0.92732700 & 1.56429300 \\
\hline 746 & $\mathrm{C}$ & & 0.79762300 & -0.56726000 & 0.33761300 \\
\hline 747 & $\mathrm{H}$ & & 0.51903300 & -1.21042700 & -0.50338300 \\
\hline 748 & $\mathrm{H}$ & & 1.03747400 & -1.23344100 & 1.17174200 \\
\hline 749 & $\mathrm{C}$ & & 2.02909300 & 0.25324400 & -0.02224100 \\
\hline 750 & $\mathrm{H}$ & & 2.26894400 & 0.92334100 & 0.80829100 \\
\hline 751 & $\mathrm{H}$ & & 1.80126700 & 0.89550700 & -0.87513400 \\
\hline 752 & $\mathrm{C}$ & & 3.23178000 & -0.62517200 & -0.34196200 \\
\hline 753 & $\mathrm{H}$ & & 4.10565400 & -0.02938400 & -0.60318600 \\
\hline 754 & $\mathrm{H}$ & & 3.49552200 & -1.25090200 & 0.51202400 \\
\hline 755 & $\mathrm{H}$ & & 3.01437900 & -1.28752000 & -1.18108600 \\
\hline 756 & $\mathrm{C}$ & & -1.56621500 & -0.61169500 & 1.17124600 \\
\hline 757 & $\mathrm{H}$ & & -1.85407900 & -1.30168300 & 0.27086900 \\
\hline 758 & $\mathrm{H}$ & & -1.28674400 & -1.30638900 & 1.96325000 \\
\hline 759 & $\mathrm{H}$ & & -2.46427300 & -0.05845700 & 1.43992800 \\
\hline 760 & $\mathrm{O}$ & & -2.23339000 & $\begin{array}{c}-1.83346900 \\
\text { S-19 }\end{array}$ & -0.98445500 \\
\hline
\end{tabular}


772

773

774

775

776

777

778

779

780

781

782

783

784

785

786

787

788

789

790

791

792

793

794

795

796

797

798

799

800

801

802

803

804

805

806
$\mathrm{H}$

Frequencies[1/cm]

$$
\begin{array}{ll}
-737.55 & 280.22
\end{array}
$$

Frequencies[1/cm]

$$
\begin{array}{llllll}
16.19 & 372.17 & 946.64 & 1214.73 & 1419.86 & 2903.55 \\
49.91 & 401.01 & 989.00 & 1239.23 & 1421.20 & 2965.71 \\
83.24 & 525.25 & 993.50 & 1252.36 & 1423.25 & 2968.42 \\
156.71 & 662.74 & 998.58 & 1274.85 & 1429.38 & 2974.45 \\
210.62 & 732.99 & 1021.39 & 1319.33 & 1651.31 & 2976.03 \\
232.35 & 810.02 & 1076.92 & 1337.54 & 2826.50 & 2978.65 \\
250.83 & 865.63 & 1102.30 & 1352.08 & 2857.41 & 2997.72 \\
276.48 & 918.69 & 1143.00 & 1384.97 & 2886.90 & 3011.17 \\
324.81 & 919.21 & 1193.94 & 1400.61 & 2895.93 & 3078.54
\end{array}
$$

TS-abs4
C

2.80503500
$-0.29016300$

0.20226200

$-1.37042100$

0.39210400

1.47621800

$-0.20892800$

$-1.29616600$

0.31183600

1.23286000

$-0.23983200$

0.19139500

$-1.31478100$

0.01684500

$-0.44558400$

$-0.38089400$

1.08720800

0.05740000

1.13136000

$-0.38509300$

$-0.36393600$ 


\begin{tabular}{|c|c|c|c|c|c|c|}
\hline 807 & \multicolumn{2}{|l|}{$\mathrm{H}$} & \multicolumn{2}{|c|}{3.63280100} & 1.20005700 & 0.41794300 \\
\hline 808 & \multicolumn{2}{|l|}{$\mathrm{H}$} & \multicolumn{2}{|c|}{2.74510700} & 0.99242800 & -1.19059900 \\
\hline 809 & \multicolumn{2}{|l|}{$\mathrm{C}$} & \multicolumn{2}{|c|}{1.90530900} & -0.00338400 & 0.45840400 \\
\hline 810 & \multicolumn{2}{|l|}{$\mathrm{H}$} & \multicolumn{2}{|c|}{1.99608400} & -0.21262400 & 1.52234000 \\
\hline 811 & \multicolumn{2}{|l|}{$\mathrm{C}$} & \multicolumn{2}{|c|}{0.73217000} & -0.63978700 & -0.22743000 \\
\hline 812 & \multicolumn{2}{|l|}{$\mathrm{H}$} & \multicolumn{2}{|c|}{0.76184800} & -0.36399100 & -1.28559100 \\
\hline 813 & \multicolumn{2}{|l|}{$\mathrm{C}$} & \multicolumn{2}{|c|}{-0.57249100} & -0.11021900 & 0.35962800 \\
\hline 814 & \multicolumn{2}{|l|}{$\mathrm{H}$} & \multicolumn{2}{|c|}{-0.55696400} & 1.04252700 & 0.24087600 \\
\hline 815 & $\mathrm{H}$ & & -0.6 & 1292100 & -0.27612100 & 1.44122000 \\
\hline 816 & $\mathrm{C}$ & & -1.8 & 4518500 & -0.57284600 & -0.32273100 \\
\hline 817 & $\mathrm{H}$ & & -1.9 & 8148100 & -1.64467100 & -0.15431300 \\
\hline 818 & $\mathrm{H}$ & & -1.7 & 3786200 & -0.43476000 & -1.40161300 \\
\hline 819 & $\mathrm{C}$ & & -3.0 & 6784100 & 0.18580500 & 0.17943700 \\
\hline 820 & $\mathrm{H}$ & & -2.9 & 5590600 & 1.25361700 & -0.00882900 \\
\hline 821 & $\mathrm{H}$ & & -3.9 & 7757600 & -0.15582900 & -0.31235500 \\
\hline 822 & $\mathrm{H}$ & & -3.1 & 9419400 & 0.04754900 & 1.25415600 \\
\hline 823 & $\mathrm{C}$ & & & 1936500 & -2.16529900 & -0.10803700 \\
\hline 824 & $\mathrm{H}$ & & & 4274100 & -2.47009700 & 0.93792300 \\
\hline 825 & $\mathrm{H}$ & & & 1690600 & -2.65010200 & -0.66204100 \\
\hline 826 & $\mathrm{H}$ & & & 7038500 & -2.52667800 & -0.49745500 \\
\hline 827 & $\mathrm{O}$ & & -0.2 & 5146500 & 2.38852800 & -0.18936600 \\
\hline 828 & $\mathrm{H}$ & & 0.70457 & 400 & .29500100 & 03821200 \\
\hline 829 & Frequencies[] & $/ \mathrm{cm}]$ & & & & \\
\hline 830 & -670.28 & 265.70 & 799.02 & 1047.27 & $1271.95 \quad 1427.56$ & $56 \quad 2925.89$ \\
\hline 831 & 49.40 & 287.09 & 820.29 & 1067.33 & $1281.48 \quad 1431.33$ & $33 \quad 2965.82$ \\
\hline 832 & 73.61 & 296.52 & 864.78 & 1104.78 & $1323.75 \quad 1451.80$ & $\begin{array}{ll}30 & 2968.65\end{array}$ \\
\hline 833 & 85.74 & 346.74 & 912.97 & 1144.40 & $1329.90 \quad 1639.83$ & $33 \quad 2978.23$ \\
\hline 834 & 95.94 & 390.67 & 923.80 & 1195.03 & $1342.94 \quad 2887.74$ & $\begin{array}{ll}74 & 2978.49\end{array}$ \\
\hline 835 & 124.27 & 494.75 & 944.73 & 1218.18 & $1384.13 \quad 2890.76$ & $\begin{array}{ll}76 \quad 2981.85\end{array}$ \\
\hline 836 & 165.67 & 661.17 & 985.01 & 1247.86 & $1407.92 \quad 2897.64$ & $64 \quad 2993.41$ \\
\hline 837 & 225.56 & 688.99 & 995.93 & 1252.74 & $1421.05 \quad 2902.90$ & $90 \quad 3076.83$ \\
\hline 838 & 239.09 & 697.13 & 1013.54 & 1263.18 & $1425.48 \quad 2904.08$ & $08 \quad 3558.24$ \\
\hline 839 & & & & & & \\
\hline 840 & bs5 & & & & & \\
\hline 841 & $\mathrm{C}$ & & 1.81 & 900800 & 1.82024200 & -0.13748300 \\
\hline 842 & $\mathrm{H}$ & & & 4083800 & 2.62802400 & 0.44498300 \\
\hline 843 & $\mathrm{H}$ & & & 8221400 & 1.95190000 & -1.21263900 \\
\hline 844 & $\mathrm{C}$ & & & 6581800 & 0.71409700 & 0.43169900 \\
\hline 845 & $\mathrm{H}$ & & & 1481500 & 0.61213100 & 1.51443300 \\
\hline 846 & $\mathrm{C}$ & & & 7780100 & -0.45897500 & -0.29420200 \\
\hline 847 & $\mathrm{H}$ & & & 6578700 & -0.22988100 & -1.36342100 \\
\hline 848 & $\mathrm{C}$ & & -0.6 & 6792100 & -0.70717700 & 0.15805300 \\
\hline 849 & $\mathrm{H}$ & & -0.6 & 5607300 & -0.91716600 & 1.24081600 \\
\hline 850 & $\mathrm{H}$ & & -1.0 & 3448700 & -1.63058400 & -0.30589100 \\
\hline 851 & $\mathrm{C}$ & & -1.6 & 0106400 & 0.40834000 & -0.14684600 \\
\hline 852 & $\mathrm{H}$ & & -1.1 & 9620000 & $\begin{array}{c}1.40651000 \\
\mathrm{~s}-21\end{array}$ & -0.24306100 \\
\hline
\end{tabular}




\begin{tabular}{|c|c|c|c|c|c|}
\hline 853 & $\mathrm{C}$ & & -3.06747100 & 0.22204800 & 0.00465500 \\
\hline 854 & $\mathrm{H}$ & & -3.35917800 & 0.13610700 & 1.06015200 \\
\hline 855 & $\mathrm{H}$ & & -3.63027600 & 1.05236600 & -0.41860400 \\
\hline 856 & $\mathrm{H}$ & & -3.40105400 & -0.69984500 & -0.47993700 \\
\hline 857 & $\mathrm{C}$ & & 1.63074900 & -1.70595200 & -0.05866400 \\
\hline 858 & $\mathrm{H}$ & & 1.66186800 & -1.94954400 & 1.00551500 \\
\hline 859 & $\mathrm{H}$ & & 1.21618400 & -2.56488000 & -0.58744400 \\
\hline 860 & $\mathrm{H}$ & & 2.65404000 & -1.55088300 & -0.39817700 \\
\hline 861 & \multicolumn{2}{|c|}{ Frequencies $[1 / \mathrm{cm}]$} & & & \\
\hline 862 & 54.07 & 368.42 & $936.83 \quad 1220.10$ & \multicolumn{2}{|c|}{$1402.79 \quad 2899.87$} \\
\hline 863 & 77.63 & 401.35 & $950.18 \quad 1251.80$ & \multicolumn{2}{|c|}{$1412.93 \quad 2901.79$} \\
\hline 864 & 96.80 & 490.26 & $991.74 \quad 1273.22$ & \multicolumn{2}{|c|}{$1421.17 \quad 2959.84$} \\
\hline 865 & 133.36 & 671.97 & $1012.04 \quad 1275.93$ & \multicolumn{2}{|c|}{$1425.74 \quad 2969.71$} \\
\hline 866 & 174.19 & 777.22 & $1048.99 \quad 1330.50$ & \multicolumn{2}{|c|}{$1645.68 \quad 2971.92$} \\
\hline 867 & 224.74 & 830.47 & $1076.58 \quad 1334.57$ & \multicolumn{2}{|c|}{$2795.68 \quad 2976.17$} \\
\hline 868 & 278.16 & 869.77 & $1100.57 \quad 1355.08$ & \multicolumn{2}{|c|}{$2839.35 \quad 2995.51$} \\
\hline 869 & 303.19 & 918.67 & $1134.14 \quad 1383.88$ & \multicolumn{2}{|c|}{$2870.73 \quad 3036.97$} \\
\hline 870 & 331.24 & 921.30 & $1177.43 \quad 1392.45$ & \multicolumn{2}{|c|}{$2894.61 \quad 3076.53$} \\
\hline \multicolumn{6}{|c|}{871} \\
\hline 872 & \multicolumn{5}{|l|}{ TS-abs5 } \\
\hline 873 & $\mathrm{C}$ & & -1.85282500 & 1.75816400 & -0.72723800 \\
\hline 874 & $\mathrm{H}$ & & -2.33324200 & 2.28415700 & -1.54120300 \\
\hline 875 & $\mathrm{H}$ & & -1.50174700 & 2.35063800 & 0.10972400 \\
\hline 876 & $\mathrm{C}$ & & -1.69235500 & 0.44406900 & -0.74601900 \\
\hline 877 & $\mathrm{H}$ & & -2.04784100 & -0.12689500 & -1.60231900 \\
\hline 878 & $\mathrm{C}$ & & -1.04088100 & -0.35975500 & 0.34034700 \\
\hline 879 & $\mathrm{H}$ & & -0.78825900 & 0.31694000 & 1.16069000 \\
\hline 880 & $\mathrm{C}$ & & 0.26430300 & -0.99473900 & -0.15541100 \\
\hline 881 & $\mathrm{H}$ & & 0.04369000 & -1.66580300 & -0.99483400 \\
\hline 882 & $\mathrm{H}$ & & 0.67199000 & -1.62615800 & 0.64074200 \\
\hline 883 & $\mathrm{C}$ & & 1.31255900 & 0.01209300 & -0.58558600 \\
\hline 884 & $\mathrm{H}$ & & 1.44412400 & 0.76437800 & 0.27486200 \\
\hline 885 & $\mathrm{H}$ & & 0.94505000 & 0.63951300 & -1.39936500 \\
\hline 886 & $\mathrm{C}$ & & 2.66318900 & -0.59342000 & -0.90877900 \\
\hline 887 & $\mathrm{H}$ & & 2.58080800 & -1.28429200 & -1.75084000 \\
\hline 888 & $\mathrm{H}$ & & 3.39234000 & 0.17136100 & -1.17117000 \\
\hline 889 & $\mathrm{H}$ & & 3.05382300 & -1.15870000 & -0.06064400 \\
\hline 890 & $\mathrm{C}$ & & -1.99770700 & -1.43733500 & 0.84992600 \\
\hline 891 & $\mathrm{H}$ & & -2.26589300 & -2.12303800 & 0.04315700 \\
\hline 892 & $\mathrm{H}$ & & -1.53649000 & -2.02156100 & 1.64708700 \\
\hline 893 & $\mathrm{H}$ & & -2.91605200 & -0.99503100 & 1.23409600 \\
\hline 894 & $\mathrm{O}$ & & 1.65992500 & 1.36684200 & 1.62369200 \\
\hline 895 & $\mathrm{H}$ & & 2.04059900 & .56529100 & .01703500 \\
\hline 896 & Frequencies[] & $/ \mathrm{cm}]$ & & & \\
\hline 897 & -610.23 & 223.88 & 1032.04 & 1288.101421 & $73 \quad 2932.82$ \\
\hline 898 & 22.97 & 276.66 & $835.42 \quad 1078.43$ & 1321.831426 & $10 \quad 2951.63$ \\
\hline
\end{tabular}




\begin{tabular}{|c|c|c|c|c|c|c|}
\hline 899 & 63.31 & 307.51 & 864.73 & 1108.57 & $1334.32 \quad 1577$ & $\begin{array}{ll}61 & 2958.20\end{array}$ \\
\hline 900 & 81.40 & 338.75 & 913.88 & 1144.99 & 1338.521646 & $.93 \quad 2968.80$ \\
\hline 901 & 92.91 & 400.80 & 925.80 & 1162.68 & 1350.332861. & $.81 \quad 2974.81$ \\
\hline 902 & 111.00 & 492.10 & 944.13 & 1198.46 & 1385.722892 & $.50 \quad 2976.52$ \\
\hline 903 & 123.33 & 585.83 & 975.70 & 1226.57 & 1403.762893 & $.44 \quad 2994.49$ \\
\hline 904 & 174.00 & 674.62 & 991.31 & 1253.58 & 1419.152897 & $\begin{array}{ll}64 & 3076.80\end{array}$ \\
\hline 905 & 218.21 & 769.19 & 1009.59 & 1278.75 & 1419.362912 & $.83 \quad 3588.64$ \\
\hline \multicolumn{7}{|l|}{906} \\
\hline 907 & \multicolumn{6}{|l|}{ P-abs6 } \\
\hline 908 & $\mathrm{C}$ & & \multicolumn{2}{|c|}{1.81025800} & 1.80311900 & -0.08856300 \\
\hline 909 & $\mathrm{H}$ & & \multicolumn{2}{|c|}{2.21789900} & 2.60294600 & 0.51459700 \\
\hline 910 & $\mathrm{H}$ & & \multicolumn{2}{|c|}{1.82013900} & 1.94024900 & -1.16363300 \\
\hline 911 & $\mathrm{C}$ & & \multicolumn{2}{|c|}{1.31956600} & 0.70063300 & 0.45595600 \\
\hline 912 & $\mathrm{H}$ & & \multicolumn{2}{|c|}{1.32327200} & 0.59410600 & 1.53951000 \\
\hline 913 & $\mathrm{C}$ & & \multicolumn{2}{|c|}{0.74897200} & -0.46388000 & -0.29814700 \\
\hline 914 & $\mathrm{H}$ & & \multicolumn{2}{|c|}{0.78522900} & -0.22893900 & -1.36641800 \\
\hline 915 & $\mathrm{C}$ & & -0.71 & 187800 & -0.71116800 & 0.09227500 \\
\hline 916 & $\mathrm{H}$ & & -0.76 & 5197400 & -0.91645500 & 1.16719500 \\
\hline 917 & $\mathrm{H}$ & & -1.06 & 5502100 & -1.61447800 & -0.41170400 \\
\hline 918 & $\mathrm{C}$ & & -1.64 & 547800 & 0.44835200 & -0.24341800 \\
\hline 919 & $\mathrm{H}$ & & -1.57 & 251600 & 0.67866700 & -1.31115500 \\
\hline 920 & $\mathrm{H}$ & & -1.28 & 372000 & 1.35163300 & 0.26798300 \\
\hline 921 & $\mathrm{C}$ & & -3.05 & 806000 & 0.17661700 & 0.12439300 \\
\hline 922 & $\mathrm{H}$ & & -3.86 & 7737200 & 0.73612800 & -0.31892400 \\
\hline 923 & $\mathrm{H}$ & & -3.28 & 793900 & -0.46114700 & 0.96602000 \\
\hline 924 & $\mathrm{C}$ & & 1.58 & 8724300 & -1.71700000 & -0.03915800 \\
\hline 925 & $\mathrm{H}$ & & $1.5^{\circ}$ & 7878900 & -1.96599900 & 1.02410800 \\
\hline 926 & $\mathrm{H}$ & & 1.18 & 8654200 & -2.57075200 & -0.58655700 \\
\hline 927 & $\mathrm{H}$ & & 2.62 & 2292500 & -1.56599200 & -0.34105100 \\
\hline 928 & Frequencies & $/ \mathrm{cm}]$ & & & & \\
\hline 929 & 68.36 & 401.67 & 921.23 & 1215.99 & 1396.952896. & \\
\hline 930 & 77.72 & 434.91 & 953.82 & 1237.95 & 1413.392924 & \\
\hline 931 & 113.33 & 496.36 & 992.50 & 1255.77 & 1422.132960 & \\
\hline 932 & 131.17 & 671.10 & 1002.76 & 1277.58 & 1426.422970 & \\
\hline 933 & 172.64 & 706.77 & 1037.27 & 1284.06 & 1646.372975 & \\
\hline 934 & 224.71 & 796.36 & 1051.13 & 1321.68 & 2834.252994 & \\
\hline 935 & 281.21 & 835.44 & 1103.87 & 1336.56 & 2875.273001 & \\
\hline 936 & 310.70 & 914.32 & 1116.26 & 1385.50 & 2889.453075 & \\
\hline 937 & 339.07 & 918.70 & 1169.24 & 1392.92 & 2892.333099 & \\
\hline 938 & & & & & & \\
\hline 939 & TS-abs6 & & & & & \\
\hline 940 & $\mathrm{C}$ & & -2.766 & 38600 & -1.33685800 & -0.55998400 \\
\hline 941 & $\mathrm{H}$ & & -3.45 & 492100 & -2.07737500 & -0.17615800 \\
\hline 942 & $\mathrm{H}$ & & -2.61 & 458000 & -1.31226200 & -1.63288700 \\
\hline 943 & $\mathrm{C}$ & & -2.13 & 541000 & -0.49115600 & 0.23975100 \\
\hline 944 & $\mathrm{H}$ & & -2.31 & .037100 & $\begin{array}{c}-0.54201700 \\
\mathrm{~s}-23\end{array}$ & 1.31323200 \\
\hline
\end{tabular}




\begin{tabular}{|c|c|c|c|c|c|c|}
\hline 945 & \multicolumn{2}{|l|}{$\mathrm{C}$} & \multicolumn{2}{|c|}{-1.17435600} & 0.57314600 & -0.20031600 \\
\hline 946 & \multicolumn{2}{|l|}{$\mathrm{H}$} & \multicolumn{2}{|c|}{-1.08196800} & 0.51959500 & -1.28960900 \\
\hline 947 & \multicolumn{2}{|l|}{$\mathrm{C}$} & \multicolumn{2}{|c|}{0.21253100} & 0.35062500 & 0.41185000 \\
\hline 948 & \multicolumn{2}{|l|}{$\mathrm{H}$} & \multicolumn{2}{|c|}{0.12624500} & 0.37788500 & 1.50385300 \\
\hline 949 & \multicolumn{2}{|l|}{$\mathrm{H}$} & \multicolumn{2}{|c|}{0.86561600} & 1.18023400 & 0.12999000 \\
\hline 950 & \multicolumn{2}{|l|}{$\mathrm{C}$} & \multicolumn{2}{|c|}{0.86901800} & -0.95718100 & -0.01166000 \\
\hline 951 & \multicolumn{2}{|l|}{$\mathrm{H}$} & \multicolumn{2}{|c|}{0.90867400} & -1.00422700 & -1.10405900 \\
\hline 952 & \multicolumn{2}{|l|}{$\mathrm{H}$} & \multicolumn{2}{|c|}{0.24919500} & -1.80167900 & 0.30311900 \\
\hline 953 & \multicolumn{2}{|l|}{$\mathrm{C}$} & \multicolumn{2}{|c|}{2.26027300} & -1.11387500 & 0.56054100 \\
\hline 954 & \multicolumn{2}{|l|}{$\mathrm{H}$} & \multicolumn{2}{|c|}{2.77492700} & -2.01538900 & 0.23359300 \\
\hline 955 & \multicolumn{2}{|l|}{$\mathrm{H}$} & \multicolumn{2}{|c|}{2.91681200} & -0.21703300 & 0.20893700 \\
\hline 956 & \multicolumn{2}{|l|}{$\mathrm{H}$} & & 8434500 & -1.04147900 & 1.64821000 \\
\hline 957 & $\mathrm{C}$ & & -1.7 & 812700 & 1.95450300 & 0.18244000 \\
\hline 958 & $\mathrm{H}$ & & -1.8 & 2407100 & 2.02935300 & 1.26572200 \\
\hline 959 & $\mathrm{H}$ & & -1.0 & 819200 & 2.73669900 & -0.13519100 \\
\hline 960 & $\mathrm{H}$ & & -2.6 & 977500 & 2.14074400 & -0.27352200 \\
\hline 961 & $\mathrm{O}$ & & & 0682000 & 0.84390000 & -0.54104600 \\
\hline 962 & $\mathrm{H}$ & & & 5824300 & 0.40053000 & -1.40259600 \\
\hline 963 & Frequencies [] & $/ \mathrm{cm}]$ & & & & \\
\hline 964 & -720.07 & 281.07 & 787.47 & 1025.80 & $1279.60 \quad 1422.08$ & $8 \quad 2926.99$ \\
\hline 965 & 29.23 & 308.24 & 835.45 & 1066.42 & $1285.82 \quad 1426.49$ & $9 \quad 2934.62$ \\
\hline 966 & 60.92 & 316.47 & 856.57 & 1116.46 & $1296.94 \quad 1432.35$ & $5 \quad 2961.43$ \\
\hline 967 & 75.74 & 349.50 & 916.85 & 1122.63 & $1326.02 \quad 1646.35$ & $5 \quad 2969.70$ \\
\hline 968 & 111.46 & 401.23 & 920.03 & 1174.29 & $1337.23 \quad 2873.36$ & $6 \quad 2975.81$ \\
\hline 969 & 120.32 & 495.41 & 942.34 & 1222.66 & $1383.05 \quad 2883.65$ & $5 \quad 2991.04$ \\
\hline 970 & 138.82 & 657.59 & 971.21 & 1241.73 & $1384.35 \quad 2890.61$ & 12994.98 \\
\hline 971 & 171.27 & 675.80 & 993.04 & 1248.75 & $1405.32 \quad 2896.73$ & $3 \quad 3076.47$ \\
\hline 972 & 224.50 & 753.18 & 999.95 & 1259.61 & $1416.28 \quad 2909.66$ & $6 \quad 3595.88$ \\
\hline 973 & & & & & & \\
\hline 974 & $\mathrm{H}_{2} \mathrm{O}$ & & & & & \\
\hline 975 & $\mathrm{O}$ & & 0.00 & 000000 & 0.00000000 & 0.11691500 \\
\hline 976 & $\mathrm{H}$ & & & 0000000 & 0.75986200 & -0.46766000 \\
\hline 977 & $\mathrm{H}$ & & 0.00000 & 000 & 75986200 & 6766000 \\
\hline 978 & Frequencies[] & $/ \mathrm{cm}]$ & & & & \\
\hline 979 & 1542.34 & 3674.86 & 3772.0 & & & \\
\hline 980 & & & & & & \\
\hline 981 & PC-abs1 & & & & & \\
\hline 982 & $\mathrm{C}$ & & -2.78 & 87600 & 0.36872100 & -0.41658400 \\
\hline 983 & $\mathrm{H}$ & & -3.7 & 572900 & 0.65184800 & -0.88952600 \\
\hline 984 & $\mathrm{H}$ & & -1.0 & 2304600 & 2.81078800 & 1.34653700 \\
\hline 985 & $\mathrm{C}$ & & -1.8 & 033500 & -0.47730400 & -0.63309700 \\
\hline 986 & $\mathrm{H}$ & & -1.8 & 965100 & -1.10711000 & -1.52750700 \\
\hline 987 & $\mathrm{C}$ & & -0.6 & 602600 & -0.67163200 & 0.24022300 \\
\hline 988 & $\mathrm{H}$ & & -0.7 & 026000 & -0.00761400 & 1.10164500 \\
\hline 989 & $\mathrm{C}$ & & & 5444200 & -0.26636700 & -0.53376300 \\
\hline 990 & $\mathrm{H}$ & & & 8769100 & $\begin{array}{c}0.71047400 \\
\text { S-24 }\end{array}$ & -0.99282700 \\
\hline
\end{tabular}




\begin{tabular}{|c|c|c|c|c|c|}
\hline 991 & $\mathrm{H}$ & & 0.81748000 & -0.97855600 & -1.35078100 \\
\hline 992 & $\mathrm{C}$ & & 1.90324800 & -0.17613400 & 0.33471900 \\
\hline 993 & $\mathrm{H}$ & & 2.13360600 & -1.15054000 & 0.76998700 \\
\hline 994 & $\mathrm{H}$ & & 1.69679100 & 0.50483500 & 1.16407900 \\
\hline 995 & $\mathrm{C}$ & & 3.10677800 & 0.32701500 & -0.45230000 \\
\hline 996 & $\mathrm{H}$ & & 2.91142000 & 1.32101200 & -0.85624900 \\
\hline 997 & $\mathrm{H}$ & & 3.99740900 & 0.38708500 & 0.17234200 \\
\hline 998 & $\mathrm{H}$ & & 3.32912500 & -0.33578900 & -1.29018000 \\
\hline 999 & $\mathrm{C}$ & & -0.54878600 & -2.12043200 & 0.72456500 \\
\hline 1000 & $\mathrm{H}$ & & -0.44153400 & -2.80101800 & -0.12307200 \\
\hline 1001 & $\mathrm{H}$ & & 0.29645900 & -2.27808800 & 1.39279000 \\
\hline 1002 & $\mathrm{H}$ & & -1.45894800 & -2.38812900 & 1.26025200 \\
\hline 1003 & $\mathrm{O}$ & & -0.57971800 & 2.58576400 & 0.52577500 \\
\hline 1004 & $\mathrm{H}$ & & -1.22974100 & 2.07149200 & 0.03373400 \\
\hline 1005 & \multicolumn{2}{|c|}{ Frequencies $[1 / \mathrm{cm}]$} & & & \\
\hline 1006 & 34.59 & 220.46 & $\begin{array}{ll}704.72 & 1039.06\end{array}$ & $1293.62 \quad 1432.48$ & $8 \quad 2923.61$ \\
\hline 1007 & 51.58 & 240.43 & $747.81 \quad 1086.45$ & $1328.56 \quad 1540.03$ & $3 \quad 2940.71$ \\
\hline 1008 & 51.77 & 294.03 & $818.38 \quad 1106.69$ & $1336.65 \quad 1605.99$ & 92964.03 \\
\hline 1009 & 81.82 & 317.83 & $835.46 \quad 1122.86$ & $1340.95 \quad 2873.08$ & $8 \quad 2964.89$ \\
\hline 1010 & 109.86 & 344.17 & $855.58 \quad 1170.75$ & $1402.80 \quad 2895.32$ & $2 \quad 2971.46$ \\
\hline 1011 & 112.89 & 372.04 & $876.33 \quad 1208.29$ & $1416.28 \quad 2895.50$ & $0 \quad 2981.00$ \\
\hline 1012 & 134.41 & 395.82 & $915.48 \quad 1236.61$ & $1421.81 \quad 2897.48$ & $8 \quad 3094.72$ \\
\hline 1013 & 157.76 & 488.63 & $965.53 \quad 1264.19$ & $1425.33 \quad 2900.32$ & $2 \quad 3619.23$ \\
\hline 1014 & 170.75 & 662.34 & $1008.18 \quad 1282.98$ & $1428.08 \quad 2920.73$ & $3 \quad 3734.25$ \\
\hline \multicolumn{6}{|l|}{1015} \\
\hline 1016 & PC-abs2 & & & & \\
\hline 1017 & $\mathrm{C}$ & & 2.53764600 & -1.37970500 & 0.93776100 \\
\hline 1018 & $\mathrm{H}$ & & 3.41217900 & -0.96135800 & -1.42149000 \\
\hline 1019 & $\mathrm{H}$ & & 2.43689600 & -2.46491900 & -0.96118300 \\
\hline 1020 & $\mathrm{C}$ & & 1.64004900 & -0.63822800 & -0.34843100 \\
\hline 1021 & $\mathrm{H}$ & & 1.91069200 & 1.64767700 & -0.15101100 \\
\hline 1022 & $\mathrm{C}$ & & 0.36955100 & -0.77618900 & 0.38798800 \\
\hline 1023 & $\mathrm{H}$ & & 0.16853700 & -1.85578000 & 0.46145700 \\
\hline 1024 & $\mathrm{C}$ & & -0.77978700 & -0.12726300 & -0.39812900 \\
\hline 1025 & $\mathrm{H}$ & & -0.78454900 & -0.53766900 & -1.41124900 \\
\hline 1026 & $\mathrm{H}$ & & -0.57340200 & 0.94304800 & -0.48712500 \\
\hline 1027 & $\mathrm{C}$ & & -2.15240100 & -0.33289000 & 0.23164700 \\
\hline 1028 & $\mathrm{H}$ & & -2.19425200 & 0.16597300 & 1.20104400 \\
\hline 1029 & $\mathrm{H}$ & & -2.30859400 & -1.39871400 & 0.42147300 \\
\hline 1030 & $\mathrm{C}$ & & -3.26803600 & 0.20158500 & -0.65797200 \\
\hline 1031 & $\mathrm{H}$ & & -3.27506600 & -0.30863800 & -1.62206800 \\
\hline 1032 & $\mathrm{H}$ & & -4.24651900 & 0.06583300 & -0.19889700 \\
\hline 1033 & $\mathrm{H}$ & & -3.13179300 & 1.26706000 & -0.84822800 \\
\hline 1034 & $\mathrm{C}$ & & 0.49895900 & -0.20377000 & 1.80366300 \\
\hline 1035 & $\mathrm{H}$ & & 0.60148400 & 0.88173900 & 1.75979800 \\
\hline 1036 & $\mathrm{H}$ & & -0.38086600 & $\begin{array}{c}-0.44394600 \\
\text { S- } 25\end{array}$ & 2.39805600 \\
\hline
\end{tabular}




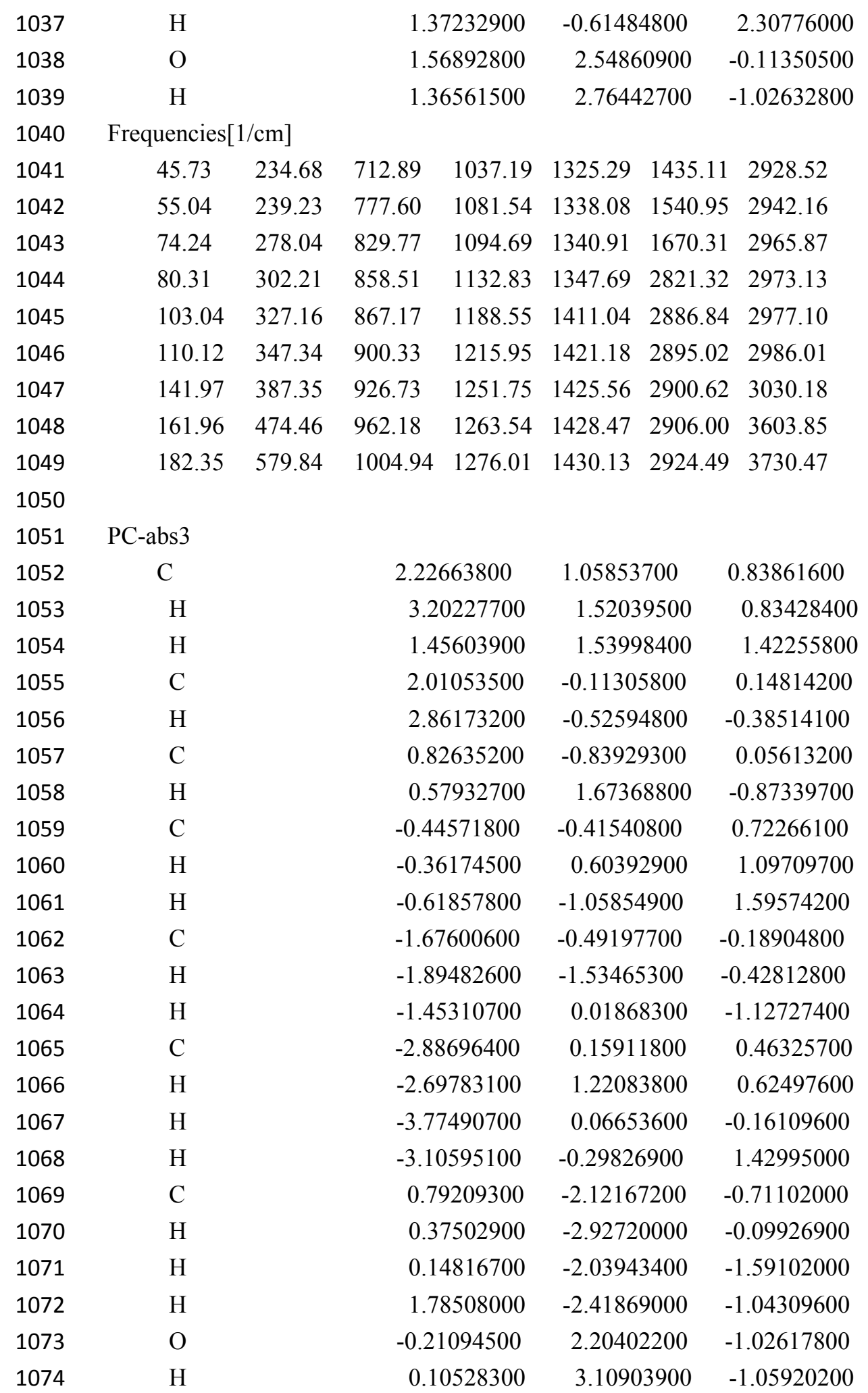

1075 Frequencies[1/cm]

$\begin{array}{llllllll}1076 & 33.62 & 220.77 & 708.17 & 1018.69 & 1335.82 & 1433.03 & 2961.41 \\ 1077 & 70.35 & 246.36 & 745.37 & 1044.85 & 1337.04 & 1448.44 & 2967.34 \\ 1078 & 85.07 & 273.31 & 761.21 & 1062.21 & 1341.84 & 1537.30 & 2973.87 \\ 1079 & 108.35 & 330.37 & 827.40 & 1150.08 & 1380.31 & 2852.49 & 2979.06 \\ 1080 & 124.56 & 339.85 & 868.99 & 1176.27 & 1393.86 & 2877.89 & 2993.38 \\ 1081 & 133.14 & 376.31 & 919.16 & 1211.89 & 1404.66 & 2897.14 & 3017.88 \\ 1082 & 157.53 & 450.04 & 945.58 & 1229.21 & 1417.28 & 2905.52 & 3104.61\end{array}$




\begin{tabular}{|c|c|c|c|c|c|c|}
\hline 1083 & 167.43 & 513.53 & 975.85 & 1263.27 & $1425.01 \quad 2920.5^{\prime}$ & $7 \quad 3629.28$ \\
\hline 1084 & 186.15 & 568.03 & 998.44 & 1298.90 & $1425.91 \quad 2940.9$ & $0 \quad 3739.61$ \\
\hline \multicolumn{7}{|l|}{1085} \\
\hline 086 & PC-abs3m & & & & & \\
\hline 1087 & $\mathrm{C}$ & & \multicolumn{2}{|c|}{2.50316100} & -1.69213100 & -0.56958900 \\
\hline 1088 & $\mathrm{H}$ & & \multicolumn{2}{|c|}{3.37517300} & -1.64945200 & -1.20775300 \\
\hline 1089 & $\mathrm{H}$ & & \multicolumn{2}{|c|}{2.29959100} & -2.63383900 & -0.07287400 \\
\hline 1090 & $\mathrm{C}$ & & \multicolumn{2}{|c|}{1.71576600} & -0.64155900 & -0.40218600 \\
\hline 1091 & $\mathrm{H}$ & & \multicolumn{2}{|c|}{1.93507000} & 0.29381700 & -0.91048800 \\
\hline 1092 & $\mathrm{C}$ & & \multicolumn{2}{|c|}{0.48377400} & -0.62760600 & 0.46444100 \\
\hline 1093 & $\mathrm{H}$ & & \multicolumn{2}{|c|}{0.34626000} & -1.64655800 & 0.85895100 \\
\hline 1094 & $\mathrm{C}$ & & \multicolumn{2}{|c|}{-0.75656600} & -0.28289400 & -0.37411200 \\
\hline 1095 & $\mathrm{H}$ & & \multicolumn{2}{|c|}{-0.77469300} & -0.94947200 & -1.23984300 \\
\hline 1096 & $\mathrm{H}$ & & \multicolumn{2}{|c|}{-0.64275200} & 0.73255900 & -0.76559800 \\
\hline 1097 & $\mathrm{C}$ & & \multicolumn{2}{|c|}{-2.07720900} & -0.41179500 & 0.37565100 \\
\hline 1098 & $\mathrm{H}$ & & \multicolumn{2}{|c|}{-2.10587000} & 0.28481600 & 1.21588000 \\
\hline 1099 & $\mathrm{H}$ & & \multicolumn{2}{|c|}{-2.14955900} & -1.41478800 & 0.80488500 \\
\hline 1100 & $\mathrm{C}$ & & \multicolumn{2}{|c|}{-3.27410800} & -0.15262200 & -0.53135700 \\
\hline 1101 & $\mathrm{H}$ & & \multicolumn{2}{|c|}{-3.29374100} & -0.86264000 & -1.35904500 \\
\hline 1102 & $\mathrm{H}$ & & \multicolumn{2}{|c|}{-4.21524500} & -0.24157500 & 0.01006800 \\
\hline 1103 & $\mathrm{H}$ & & \multicolumn{2}{|c|}{-3.22567200} & 0.84989000 & -0.95888700 \\
\hline 1104 & $\mathrm{C}$ & & \multicolumn{2}{|c|}{0.67173400} & 0.29885500 & 1.62076700 \\
\hline 1105 & $\mathrm{H}$ & & \multicolumn{2}{|c|}{1.15058900} & 2.20953100 & 0.32345700 \\
\hline 1106 & $\mathrm{H}$ & & \multicolumn{2}{|c|}{-0.17475900} & 0.75468300 & 2.11404700 \\
\hline 1107 & $\mathrm{H}$ & & 1.6 & 2193000 & 0.31854600 & 2.13561100 \\
\hline 1108 & $\mathrm{O}$ & & & 2044200 & 2.71005200 & -0.49735400 \\
\hline 1109 & $\mathrm{H}$ & & & 9083600 & 3.33258600 & -0.47127000 \\
\hline 1110 & Frequencies[ & $/ \mathrm{cm}]$ & & & & \\
\hline 1111 & 33.72 & 222.69 & 674.07 & 1012.24 & $1266.12 \quad 1434.2$ & 42965.16 \\
\hline 1112 & 47.13 & 235.54 & 706.93 & 1044.31 & $1280.01 \quad 1540.0$ & $6 \quad 2971.79$ \\
\hline 1113 & 65.17 & 300.95 & 806.22 & 1053.96 & $1327.79 \quad 1643.8$ & $2 \quad 2991.39$ \\
\hline 1114 & 80.59 & 318.90 & 853.35 & 1108.00 & $1340.78 \quad 2811.9$ & $3 \quad 2996.87$ \\
\hline 1115 & 86.72 & 333.98 & 863.52 & 1134.96 & $1381.11 \quad 2887.6$ & 12999.65 \\
\hline 1116 & 120.30 & 341.39 & 898.97 & 1183.97 & $1388.55 \quad 2896.7$ & $4 \quad 3076.99$ \\
\hline 1117 & 130.22 & 395.02 & 922.32 & 1194.76 & $1412.43 \quad 2900.6$ & $8 \quad 3093.85$ \\
\hline 1118 & 147.44 & 489.41 & 968.89 & 1244.38 & $1421.06 \quad 2919.0$ & 23609.61 \\
\hline 1119 & 169.60 & 569.48 & 1007.32 & 1257.92 & $1426.08 \quad 2938.6$ & $5 \quad 3735.92$ \\
\hline 1120 & & & & & & \\
\hline 1121 & PC-abs4 & & & & & \\
\hline 1122 & $\mathrm{C}$ & & 2.70 & 525300 & 0.81845300 & 0.17864300 \\
\hline 1123 & $\mathrm{H}$ & & 3.4 & 8638800 & 1.20266900 & 0.82020900 \\
\hline 1124 & $\mathrm{H}$ & & & 8362500 & 1.17558600 & -0.84516500 \\
\hline 1125 & $\mathrm{C}$ & & 1.8 & 1395300 & -0.05956300 & 0.61663000 \\
\hline 1126 & $\mathrm{H}$ & & 1.8 & 6434300 & -0.40521200 & 1.64682900 \\
\hline 1127 & $\mathrm{C}$ & & 0.7 & 1249200 & -0.65798600 & -0.21235200 \\
\hline 1128 & $\mathrm{H}$ & & & 0314300 & $\begin{array}{c}-0.16024300 \\
\text { S-27 }\end{array}$ & -1.18657000 \\
\hline
\end{tabular}




\begin{tabular}{|c|c|c|c|c|c|c|}
\hline 1129 & \multicolumn{2}{|l|}{$\mathrm{C}$} & \multicolumn{2}{|c|}{-0.61894200} & -0.45123000 & 0.43764300 \\
\hline 1130 & \multicolumn{2}{|l|}{$\mathrm{H}$} & \multicolumn{2}{|c|}{-0.85535100} & 2.09317400 & -0.05314900 \\
\hline 1131 & \multicolumn{2}{|l|}{$\mathrm{H}$} & \multicolumn{2}{|c|}{-0.69174500} & -0.61473400 & 1.50838200 \\
\hline 1132 & \multicolumn{2}{|l|}{$\mathrm{C}$} & \multicolumn{2}{|c|}{-1.87155100} & -0.44834800 & -0.36763300 \\
\hline 1133 & \multicolumn{2}{|l|}{$\mathrm{H}$} & \multicolumn{2}{|c|}{-2.09124100} & -1.46369400 & -0.72748900 \\
\hline 1134 & \multicolumn{2}{|l|}{$\mathrm{H}$} & \multicolumn{2}{|c|}{-1.71309800} & 0.15145500 & -1.26983000 \\
\hline 1135 & \multicolumn{2}{|l|}{$\mathrm{C}$} & \multicolumn{2}{|c|}{-3.07814100} & 0.07296200 & 0.40739800 \\
\hline 1136 & \multicolumn{2}{|l|}{$\mathrm{H}$} & \multicolumn{2}{|c|}{-2.91861700} & 1.10160000 & 0.73570500 \\
\hline 1137 & \multicolumn{2}{|l|}{$\mathrm{H}$} & \multicolumn{2}{|c|}{-3.98080100} & 0.05145600 & -0.20104800 \\
\hline 1138 & \multicolumn{2}{|l|}{$\mathrm{H}$} & \multicolumn{2}{|c|}{-3.25515600} & -0.53165300 & 1.29734400 \\
\hline 1139 & \multicolumn{2}{|l|}{$\mathrm{C}$} & \multicolumn{2}{|c|}{0.98536600} & -2.15924500 & -0.43601200 \\
\hline 1140 & \multicolumn{2}{|l|}{$\mathrm{H}$} & & 03094900 & -2.68177500 & 0.52076700 \\
\hline 1141 & $\mathrm{H}$ & & & 19138000 & -2.60893800 & -1.03188600 \\
\hline 1142 & $\mathrm{H}$ & & & 93431900 & -2.30354700 & -0.95218300 \\
\hline 1143 & $\mathrm{O}$ & & -0.1 & 1634400 & 2.51666300 & -0.49704900 \\
\hline 1144 & $\mathrm{H}$ & & & 65202300 & 2.17028800 & -0.03142500 \\
\hline 1145 & Frequencies[ & $/ \mathrm{cm}]$ & & & & \\
\hline 1146 & 23.36 & 228.79 & 670.90 & 1003.46 & $1285.82 \quad 1428.3$ & $31 \quad 2967.82$ \\
\hline 1147 & 47.83 & 235.09 & 740.34 & 1021.62 & $1321.76 \quad 1545.4$ & $41 \quad 2974.93$ \\
\hline 1148 & 62.65 & 277.22 & 814.25 & 1074.31 & $1343.38 \quad 1638.88$ & $\begin{array}{ll}38 & 2979.02\end{array}$ \\
\hline 1149 & 76.74 & 291.83 & 861.53 & 1107.70 & $1356.10 \quad 2828.40$ & $46 \quad 2979.58$ \\
\hline 1150 & 104.56 & 326.89 & 918.61 & 1140.50 & $1385.05 \quad 2891.13$ & $\begin{array}{ll}3 \quad 2992.37\end{array}$ \\
\hline 1151 & 126.30 & 367.09 & 928.95 & 1195.48 & $1401.93 \quad 2895.97$ & $97 \quad 2993.94$ \\
\hline 1152 & 146.03 & 403.99 & 949.19 & 1212.91 & $1419.79 \quad 2899.29$ & $29 \quad 3076.69$ \\
\hline 1153 & 167.40 & 438.33 & 989.78 & 1242.06 & 1421.462900 .3 & $31 \quad 3640.26$ \\
\hline 1154 & 194.70 & 530.11 & 994.97 & 1254.92 & $1422.16 \quad 2960.95$ & 993725.79 \\
\hline 1155 & & & & & & \\
\hline 1156 & PC-abs5 & & & & & \\
\hline 1157 & $\mathrm{C}$ & & 3.42 & 2647600 & -0.45427800 & -0.30820600 \\
\hline 1158 & $\mathrm{H}$ & & & 23875700 & -0.34204000 & -1.01324100 \\
\hline 1159 & $\mathrm{H}$ & & & 62491100 & -1.03379700 & 0.58589400 \\
\hline 1160 & $\mathrm{C}$ & & & 23919700 & 0.08995400 & -0.52254700 \\
\hline 1161 & $\mathrm{H}$ & & & 07117900 & 0.66695600 & -1.42963500 \\
\hline 1162 & $\mathrm{C}$ & & & 5962600 & -0.01484400 & 0.39776300 \\
\hline 1163 & $\mathrm{H}$ & & & 35290100 & -0.61481700 & 1.26450500 \\
\hline 1164 & $\mathrm{C}$ & & -0.0 & 9935200 & -0.72802300 & -0.31228500 \\
\hline 1165 & $\mathrm{H}$ & & & 27315800 & -1.67971900 & -0.71865700 \\
\hline 1166 & $\mathrm{H}$ & & -0.4 & 0906500 & -0.13626800 & -1.18274600 \\
\hline 1167 & $\mathrm{C}$ & & -1.2 & 8208200 & -0.98759900 & 0.55526400 \\
\hline 1168 & $\mathrm{H}$ & & -2.1 & 2681400 & 1.11239300 & 0.06362300 \\
\hline 1169 & $\mathrm{H}$ & & -1.1 & 1196600 & -1.13656200 & 1.61546100 \\
\hline 1170 & $\mathrm{C}$ & & -2.5 & 4007000 & -1.50905300 & -0.04611100 \\
\hline 1171 & $\mathrm{H}$ & & -2.4 & 1708400 & -2.54732000 & -0.38147900 \\
\hline 1172 & $\mathrm{H}$ & & -3.3 & 6866500 & -1.49321800 & 0.66037300 \\
\hline 1173 & $\mathrm{H}$ & & -2.8 & 2548500 & -0.92651900 & -0.92514900 \\
\hline 1174 & $\mathrm{C}$ & & & 54250300 & $\begin{array}{c}1.37807400 \\
\text { s-28 }\end{array}$ & 0.87509800 \\
\hline
\end{tabular}




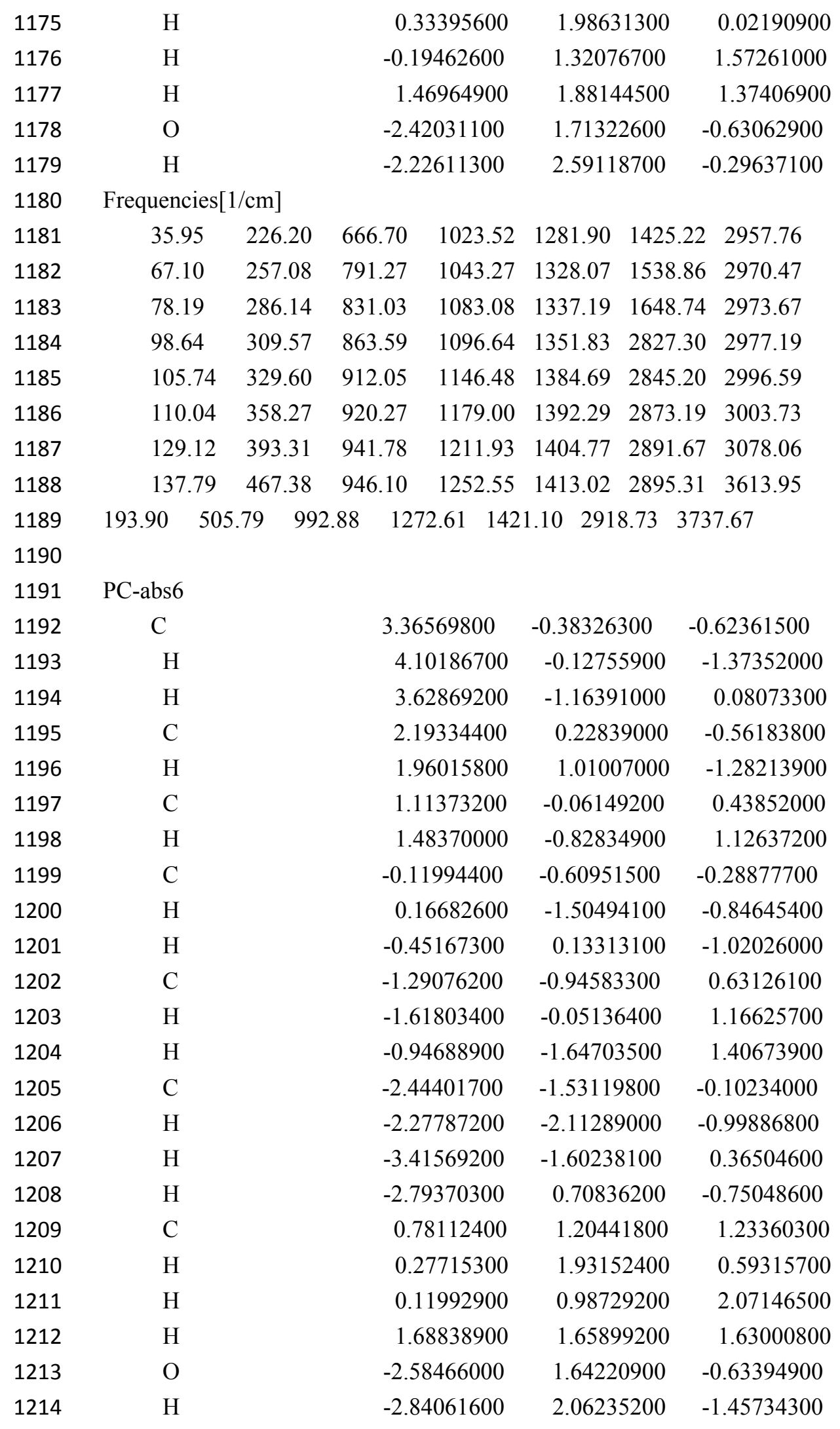

1215 Frequencies[1/cm]

$\begin{array}{llllllll}1216 & 28.55 & 225.20 & 669.31 & 1008.94 & 1271.79 & 1431.76 & 2967.36\end{array}$

$\begin{array}{llllllll}1217 & 41.69 & 233.84 & 717.84 & 1044.26 & 1293.40 & 1538.00 & 2975.05\end{array}$

$\begin{array}{llllllll}1218 & 73.91 & 295.28 & 800.45 & 1059.28 & 1331.28 & 1646.88 & 2977.71\end{array}$

$\begin{array}{lllllllll}1219 & 82.93 & 318.95 & 850.62 & 1091.51 & 1338.28 & 2820.11 & 2993.72\end{array}$

$\begin{array}{llllllll}1220 & 117.11 & 323.41 & 891.60 & 1133.24 & 1384.17 & 2883.73 & 2994.93\end{array}$ 


\begin{tabular}{|c|c|c|c|c|c|c|}
\hline 1221 & 131.23 & 345.08 & 916.86 & 1165.06 & $1391.47 \quad 2889.80$ & $\begin{array}{ll}0 & 3075.29\end{array}$ \\
\hline 1222 & 138.13 & 390.54 & 925.85 & 1223.47 & $1397.88 \quad 2899.10$ & $0 \quad 3090.08$ \\
\hline 1223 & 164.10 & 501.80 & 948.83 & 1239.71 & $1412.41 \quad 2918.04$ & $4 \quad 3613.51$ \\
\hline 1224 & 181.15 & 528.22 & 991.27 & 1256.71 & $1420.59 \quad 2935.03$ & $3 \quad 3739.40$ \\
\hline \multicolumn{7}{|l|}{1225} \\
\hline 1226 & \multicolumn{6}{|l|}{ 2-MP } \\
\hline 1227 & \multicolumn{2}{|l|}{ C } & \multicolumn{2}{|c|}{0.60647600} & 0.70432900 & 0.10032000 \\
\hline 1228 & $\mathrm{H}$ & & \multicolumn{2}{|c|}{0.63080300} & 0.86150600 & 1.18476300 \\
\hline 1229 & $\mathrm{H}$ & & \multicolumn{2}{|c|}{0.92219300} & 1.64870100 & -0.35084400 \\
\hline 1230 & $\mathrm{C}$ & & \multicolumn{2}{|c|}{1.58712600} & -0.39929700 & -0.27897100 \\
\hline 1231 & $\mathrm{H}$ & & \multicolumn{2}{|c|}{1.54249400} & -0.56005100 & -1.35875500 \\
\hline 1232 & $\mathrm{H}$ & & \multicolumn{2}{|c|}{1.27553300} & -1.34132100 & 0.17975600 \\
\hline 1233 & $\mathrm{C}$ & & \multicolumn{2}{|c|}{3.01413000} & -0.07105800 & 0.13932800 \\
\hline 1234 & $\mathrm{H}$ & & \multicolumn{2}{|c|}{3.70517700} & -0.86559400 & -0.13861200 \\
\hline 1235 & $\mathrm{H}$ & & \multicolumn{2}{|c|}{3.35474200} & 0.85085300 & -0.33419800 \\
\hline 1236 & $\mathrm{H}$ & & \multicolumn{2}{|c|}{3.08010200} & 0.06796100 & 1.21934200 \\
\hline 1237 & $\mathrm{C}$ & & -1.75 & 873900 & 1.58768000 & 0.00881300 \\
\hline 1238 & $\mathrm{H}$ & & -2.77 & 872800 & 1.39908200 & -0.32355600 \\
\hline 1239 & $\mathrm{H}$ & & -1.77 & 733900 & 1.76442900 & 1.08628400 \\
\hline 1240 & $\mathrm{H}$ & & -1.40 & 284800 & 2.49844200 & -0.47169300 \\
\hline 1241 & $\mathrm{C}$ & & -0.83 & 404300 & 0.41684900 & -0.32820300 \\
\hline 1242 & $\mathrm{H}$ & & -0.87 & 124400 & 0.19921400 & -1.39694800 \\
\hline 1243 & $\mathrm{C}$ & & -1.33 & 281500 & -0.80666800 & 0.39423800 \\
\hline 1244 & $\mathrm{H}$ & & -1.28 & 542200 & -0.72859400 & 1.50316500 \\
\hline 1245 & $\mathrm{O}$ & & -1.76 & 103400 & -1.79820400 & -0.12648200 \\
\hline 1246 & Frequencies[] & $/ \mathrm{cm}]$ & & & & \\
\hline 1247 & 49.97 & 409.55 & 1007.28 & 1288.34 & $1426.92 \quad 2927.34$ & \\
\hline 1248 & 72.70 & 536.44 & 1028.04 & 1326.71 & $1433.93 \quad 2935.23$ & \\
\hline 1249 & 105.86 & 699.81 & 1089.33 & 1334.92 & $1772.31 \quad 2964.10$ & \\
\hline 1250 & 162.57 & 802.03 & 1117.10 & 1342.65 & $2743.96 \quad 2965.77$ & \\
\hline 1251 & 209.22 & 838.13 & 1128.48 & 1352.21 & $2874.01 \quad 2975.28$ & \\
\hline 1252 & 235.96 & 867.47 & 1189.00 & 1408.36 & $2894.96 \quad 2980.16$ & \\
\hline 1253 & 287.39 & 905.98 & 1226.02 & 1417.90 & 2898.41 & \\
\hline 1254 & 324.58 & 941.28 & 1256.86 & 1423.73 & 2901.07 & \\
\hline 1255 & 345.56 & 977.83 & 1275.90 & 1425.49 & 2911.77 & \\
\hline 1256 & & & & & & \\
\hline 1257 & RC-abs1 & & & & & \\
\hline 1258 & $\mathrm{C}$ & & -1.067 & 91900 & 0.42814700 & 0.08432300 \\
\hline 1259 & $\mathrm{H}$ & & -0.83 & 744500 & 1.40858300 & -0.34332700 \\
\hline 1260 & $\mathrm{H}$ & & -0.93 & 231500 & 0.52006800 & 1.16649500 \\
\hline 1261 & $\mathrm{C}$ & & -2.52 & 296400 & 0.08833300 & -0.21738500 \\
\hline 1262 & $\mathrm{H}$ & & -2.79 & 098300 & -0.84896300 & 0.27245500 \\
\hline 1263 & $\mathrm{H}$ & & -2.63 & 589700 & -0.07759300 & -1.29216700 \\
\hline 1264 & $\mathrm{C}$ & & -3.47 & 070700 & 1.18953400 & 0.24045300 \\
\hline 1265 & $\mathrm{H}$ & & -3.24 & 1656400 & 2.13069600 & -0.26307100 \\
\hline 1266 & $\mathrm{H}$ & & -4.50 & 842400 & $\begin{array}{c}0.93491600 \\
\text { s-30 }\end{array}$ & 0.02973900 \\
\hline
\end{tabular}




\begin{tabular}{|c|c|c|c|c|c|}
\hline 1267 & $\mathrm{H}$ & & -3.37852100 & 1.35799100 & 1.31418600 \\
\hline 1268 & $\mathrm{C}$ & & -0.22295200 & -1.98934900 & 0.15641000 \\
\hline 1269 & $\mathrm{H}$ & & -0.16255800 & -1.93013100 & 1.24363400 \\
\hline 1270 & $\mathrm{H}$ & & -1.18021100 & -2.42998800 & -0.11424300 \\
\hline 1271 & $\mathrm{H}$ & & 0.57158800 & -2.65258600 & -0.18006300 \\
\hline 1272 & $\mathrm{C}$ & & -0.06798700 & -0.60703700 & -0.45520600 \\
\hline 1273 & $\mathrm{H}$ & & -0.20787400 & -0.65552500 & -1.54308700 \\
\hline 1274 & $\mathrm{C}$ & & 1.31600000 & -0.06009000 & -0.25419300 \\
\hline 1275 & $\mathrm{H}$ & & 1.51002200 & 0.93686900 & -0.69208700 \\
\hline 1276 & $\mathrm{O}$ & & 2.19203300 & -0.62218800 & 0.35604000 \\
\hline 1277 & $\mathrm{O}$ & & 4.10450100 & 1.42363300 & -0.00383700 \\
\hline 1278 & $\mathrm{H}$ & & 3.64608600 & 0.59688300 & 0.25749000 \\
\hline 1279 & \multicolumn{2}{|c|}{ Frequencies $[1 / \mathrm{cm}]$} & & & \\
\hline 1280 & 34.09 & 292.08 & $819.60 \quad 1137.38$ & 1366.972864 & $.57 \quad 2981.36$ \\
\hline 1281 & 56.13 & 322.04 & $863.53 \quad 1191.33$ & 1407.612880 & $.16 \quad 2995.00$ \\
\hline 1282 & 64.44 & 357.21 & $897.13 \quad 1224.40$ & 1419.822896 & .66 3408.70 \\
\hline 1283 & 78.55 & 404.09 & $929.29 \quad 1259.94$ & 1420.262902 & \\
\hline 1284 & 106.91 & 429.02 & $970.29 \quad 1271.18$ & 1424.192914 & \\
\hline 1285 & 155.08 & 586.07 & $1013.02 \quad 1302.57$ & $1427.03 \quad 2915$ & \\
\hline 1286 & 195.45 & 653.30 & $1032.14 \quad 1328.72$ & 1434.702940 & \\
\hline 1287 & 207.38 & 709.40 & $1080.08 \quad 1340.36$ & 1740.142967. & \\
\hline 1288 & 239.50 & 790.45 & $1115.51 \quad 1344.70$ & $2817.48 \quad 2976$ & \\
\hline \multicolumn{6}{|l|}{1289} \\
\hline 1290 & $\mathrm{RC}-\mathrm{abs} 23$ & & & & \\
\hline 1291 & $\mathrm{C}$ & & -1.87828700 & -0.30901100 & -0.52923000 \\
\hline 1292 & $\mathrm{H}$ & & -2.30595600 & -1.11432600 & -1.15783200 \\
\hline 1293 & $\mathrm{C}$ & & -0.66148200 & -0.66765000 & 0.28006000 \\
\hline 1294 & $\mathrm{H}$ & & -0.71558400 & -0.08939100 & 1.20549300 \\
\hline 1295 & $\mathrm{C}$ & & 0.57369000 & -0.18091500 & -0.50063000 \\
\hline 1296 & $\mathrm{H}$ & & 0.41520600 & 0.85906100 & -0.79855900 \\
\hline 1297 & $\mathrm{H}$ & & 0.67419800 & -0.76341100 & -1.42291300 \\
\hline 1298 & $\mathrm{C}$ & & 1.86414600 & -0.26238200 & 0.30630500 \\
\hline 1299 & $\mathrm{H}$ & & 2.08451600 & -1.30109500 & 0.55944400 \\
\hline 1300 & $\mathrm{H}$ & & 1.71857100 & 0.27324200 & 1.24750000 \\
\hline 1301 & $\mathrm{C}$ & & 3.03972500 & 0.33971300 & -0.45264000 \\
\hline 1302 & $\mathrm{H}$ & & 2.85443800 & 1.39118400 & -0.67438000 \\
\hline 1303 & $\mathrm{H}$ & & 3.96094800 & 0.27486200 & 0.12477800 \\
\hline 1304 & $\mathrm{H}$ & & 3.19965100 & -0.17893700 & -1.39912300 \\
\hline 1305 & $\mathrm{C}$ & & -0.62767400 & -2.16210900 & 0.57595900 \\
\hline 1306 & $\mathrm{H}$ & & -0.51955500 & -2.73464100 & -0.34771400 \\
\hline 1307 & $\mathrm{H}$ & & 0.20881000 & -2.41201900 & 1.22492000 \\
\hline 1308 & $\mathrm{H}$ & & -1.54136900 & -2.48991900 & 1.07238100 \\
\hline 1309 & $\mathrm{O}$ & & -0.45074400 & 2.41739000 & 0.79076300 \\
\hline 1310 & $\mathrm{H}$ & & -1.28051700 & 2.08849900 & 0.38438800 \\
\hline 1311 & $\mathrm{O}$ & & -2.37601400 & 0.78898600 & -0.55293000 \\
\hline
\end{tabular}

1312 Frequencies[1/cm] 


\begin{tabular}{|c|c|c|c|c|c|c|c|}
\hline 1313 & 23.37 & 264.79 & 825.48 & 1131.01 & \multicolumn{3}{|c|}{$1359.14 \quad 2875.83$} \\
\hline 1314 & 57.71 & 315.99 & 866.54 & 1194.01 & \multicolumn{3}{|c|}{$\begin{array}{lll}1408.68 & 2898.53 & 2989.06\end{array}$} \\
\hline 1315 & 65.82 & 375.45 & 903.81 & 1232.28 & \multicolumn{3}{|c|}{$\begin{array}{lll}1420.30 & 2899.85 & 3413.91\end{array}$} \\
\hline 1316 & 103.08 & 401.99 & 943.18 & 1266.18 & \multicolumn{3}{|c|}{$1423.95 \quad 2902.96$} \\
\hline 1317 & 127.07 & 477.71 & 971.84 & 1274.01 & \multicolumn{3}{|c|}{$1426.26 \quad 2918.39$} \\
\hline 1318 & 162.94 & 547.57 & 1011.61 & 1290.40 & \multicolumn{3}{|c|}{$1429.90 \quad 2931.08$} \\
\hline 1319 & 169.70 & 573.95 & 1041.04 & 1327.06 & \multicolumn{3}{|c|}{$1436.85 \quad 2940.28$} \\
\hline 1320 & 204.40 & 709.25 & 1098.20 & 1341.35 & \multicolumn{3}{|c|}{$1743.98 \quad 2963.19$} \\
\hline 1321 & 239.89 & 812.96 & 1118.41 & 1344.70 & \multicolumn{3}{|c|}{$2796.64 \quad 2967.22$} \\
\hline \multicolumn{8}{|l|}{1322} \\
\hline 1323 & \multicolumn{7}{|l|}{ RC-abs $2 \mathrm{~m} 45$} \\
\hline 1324 & \multicolumn{2}{|l|}{$\mathrm{C}$} & \multicolumn{2}{|c|}{2.22663900} & \multicolumn{2}{|c|}{-0.01386500} & -0.51879200 \\
\hline 1325 & $\mathrm{H}$ & & \multicolumn{2}{|c|}{2.09572700} & \multicolumn{2}{|c|}{0.66250900} & -1.39091800 \\
\hline 1326 & $\mathrm{C}$ & & \multicolumn{2}{|c|}{1.08065900} & \multicolumn{2}{|c|}{0.00283700} & 0.45732900 \\
\hline 1327 & $\mathrm{H}$ & & \multicolumn{2}{|c|}{1.38324100} & \multicolumn{2}{|c|}{-0.59678100} & 1.31778800 \\
\hline 1328 & $\mathrm{C}$ & & \multicolumn{2}{|c|}{-0.12566500} & \multicolumn{2}{|c|}{-0.65151700} & -0.22975100 \\
\hline 1329 & $\mathrm{H}$ & & 0.15 & 5150000 & -1.6614 & 8600 & -0.54406500 \\
\hline 1330 & $\mathrm{H}$ & & -0.36 & 810100 & -0.08812 & 2800 & -1.13687200 \\
\hline 1331 & $\mathrm{C}$ & & -1.36 & 835000 & -0.73005 & 000 & 0.64927000 \\
\hline 1332 & $\mathrm{H}$ & & -1.67 & 659400 & 0.2769 & 0600 & 0.94207100 \\
\hline 1333 & $\mathrm{H}$ & & -1.12 & 440400 & -1.25347 & 7900 & 1.57731100 \\
\hline 1334 & $\mathrm{C}$ & & -2.51 & 772300 & -1.43980 & 100 & -0.05667500 \\
\hline 1335 & $\mathrm{H}$ & & -2.25 & 399600 & -2.47273 & 3700 & -0.28567900 \\
\hline 1336 & $\mathrm{H}$ & & -3.42 & 003500 & $-1.4544 \mathrm{C}$ & 9400 & 0.55442300 \\
\hline 1337 & $\mathrm{H}$ & & -2.75 & 179200 & -0.9519 & 4300 & -1.00564300 \\
\hline 1338 & $\mathrm{C}$ & & 0.80 & 138000 & 1.4466 & 3700 & 0.87583400 \\
\hline 1339 & $\mathrm{H}$ & & 0.43 & 3729700 & 2.0287 & 7400 & 0.02709900 \\
\hline 1340 & $\mathrm{H}$ & & 0.0 & 4232400 & 1.4890 & 0300 & 1.65456400 \\
\hline 1341 & $\mathrm{H}$ & & 1.7 & 0168800 & 1.9236 & 0500 & 1.26309000 \\
\hline 1342 & $\mathrm{O}$ & & -2.18 & 353500 & 1.8256 & 1500 & -0.75410000 \\
\hline 1343 & $\mathrm{H}$ & & -3.02 & 068300 & 1.3401 & 0600 & -0.65829500 \\
\hline 1344 & $\mathrm{O}$ & & 3.2 & 1130800 & -0.6915 & 2700 & -0.41817000 \\
\hline 1345 & Frequencies[1] & /cm] & & & & & \\
\hline 1346 & 22.74 & 244.40 & 835.75 & 1129.16 & 1354.55 & 2879.3 & $36 \quad 2970.11$ \\
\hline 1347 & 41.40 & 267.85 & 860.06 & 1193.49 & 1406.25 & 2888.3 & $36 \quad 2984.27$ \\
\hline 1348 & 47.41 & 290.74 & 900.96 & 1218.27 & 1418.74 & 2894.5 & $\begin{array}{ll}58 \quad 3567.63\end{array}$ \\
\hline 1349 & 87.77 & 322.46 & 943.16 & 1260.31 & 1423.95 & 2902.3 & \\
\hline 1350 & 93.67 & 363.61 & 964.44 & 1267.71 & 1424.94 & 2909.7 & \\
\hline 1351 & 112.07 & 381.95 & 1007.47 & 1291.33 & 1431.29 & 2922.6 & \\
\hline 1352 & 151.22 & 551.88 & 1040.33 & 1330.06 & 1438.61 & 2934.4 & \\
\hline 1353 & 161.13 & 710.94 & 1094.21 & 1337.26 & 1770.95 & 2958.0 & \\
\hline 1354 & 208.35 & 810.87 & 1118.03 & 1340.31 & 2756.55 & 2968.8 & \\
\hline 1355 & & & & & & & \\
\hline 1356 & -ada & & & & & & \\
\hline 1357 & $\mathrm{C}$ & & -0.572 & 50100 & -0.18308 & 300 & 0.50115900 \\
\hline 1358 & $\mathrm{H}$ & & -0.41 & 527000 & $\begin{array}{r}0.8564 \\
S-\end{array}$ & $\begin{array}{l}6000 \\
32\end{array}$ & 0.80121100 \\
\hline
\end{tabular}




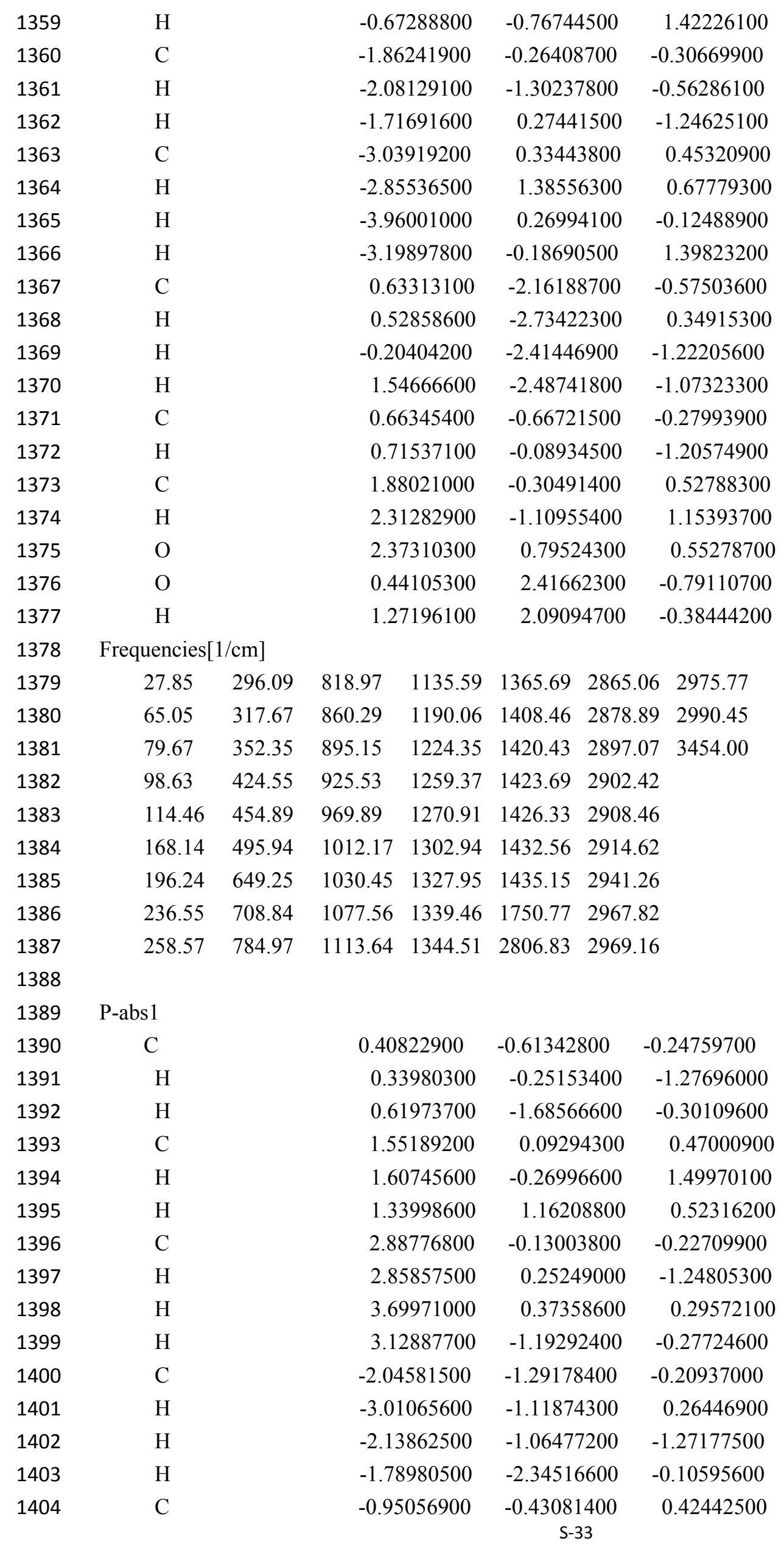




\begin{tabular}{|c|c|c|c|c|c|c|}
\hline 1405 & \multicolumn{2}{|l|}{$\mathrm{H}$} & \multicolumn{2}{|c|}{-0.88548200} & -0.67089200 & 1.48785100 \\
\hline 1406 & \multicolumn{2}{|l|}{$\mathrm{C}$} & \multicolumn{2}{|c|}{-1.43154000} & 1.01719700 & 0.34282700 \\
\hline 1407 & \multicolumn{2}{|l|}{$\mathrm{O}$} & \multicolumn{2}{|c|}{-1.03617100} & 1.86838000 & -0.36362400 \\
\hline 1408 & \multicolumn{2}{|c|}{ Frequencies $[1 / \mathrm{cm}]$} & & & & \\
\hline 1409 & 63.32 & 442.07 & 1018.98 & 1324.50 & \multicolumn{2}{|l|}{$\begin{array}{ll}1871.81 & 2974.79\end{array}$} \\
\hline 1410 & 75.57 & 589.78 & 1043.33 & 1337.69 & \multicolumn{2}{|l|}{$2884.76 \quad 2978.61$} \\
\hline 1411 & 105.49 & 709.45 & 1086.21 & 1341.76 & \multicolumn{2}{|l|}{$2896.59 \quad 2986.89$} \\
\hline 1412 & 163.29 & 737.15 & 1118.33 & & \multicolumn{2}{|l|}{2901.34} \\
\hline 1413 & 219.21 & 836.92 & 1189.20 & 1419.05 & \multicolumn{2}{|l|}{2907.69} \\
\hline 1414 & 234.61 & 843.14 & 1215.47 & 1420.34 & \multicolumn{2}{|l|}{2914.62} \\
\hline 1415 & 248.29 & 894.55 & 1243.27 & 1425.91 & \multicolumn{2}{|l|}{2927.20} \\
\hline 1416 & 332.27 & 959.00 & 1266.25 & 1426.88 & 2943.08 & \\
\hline 1417 & 374.53 & 998.08 & 1285.48 & 1433.11 & 2967.07 & \\
\hline 1418 & & & & & & \\
\hline 1419 & TS-abs1 & & & & & \\
\hline 1420 & $\mathrm{C}$ & & 1.16 & 513100 & 0.62948000 & 0.21674300 \\
\hline 1421 & $\mathrm{H}$ & & 1.1 & 7650700 & 0.35959100 & 1.27601000 \\
\hline 1422 & $\mathrm{H}$ & & 1.5 & 5848400 & 1.64722600 & 0.14460800 \\
\hline 1423 & $\mathrm{C}$ & & 2.0 & 7021300 & -0.31976600 & -0.56006100 \\
\hline 1424 & $\mathrm{H}$ & & 2.0 & 5287400 & -0.04561800 & -1.61832200 \\
\hline 1425 & $\mathrm{H}$ & & 1.6 & 7480100 & -1.33410100 & -0.48877500 \\
\hline 1426 & $\mathrm{C}$ & & 3.5 & 0342800 & -0.29128900 & -0.04532300 \\
\hline 1427 & $\mathrm{H}$ & & 3.5 & 4242200 & -0.59209700 & 1.00230000 \\
\hline 1428 & $\mathrm{H}$ & & 4.1 & 4508200 & -0.96542900 & -0.61119200 \\
\hline 1429 & $\mathrm{H}$ & & 3.9 & 2395100 & 0.71288200 & -0.11843800 \\
\hline 1430 & $\mathrm{C}$ & & -1.11 & 1309100 & 1.76316400 & 0.35689700 \\
\hline 1431 & $\mathrm{H}$ & & -2.1 & 5894400 & 1.69395000 & 0.05917700 \\
\hline 1432 & $\mathrm{H}$ & & -1.0 & 5852600 & 1.70999100 & 1.44505900 \\
\hline 1433 & $\mathrm{H}$ & & -0.72 & 2286100 & 2.72979800 & 0.04254700 \\
\hline 1434 & $\mathrm{C}$ & & -0.27 & 7847700 & 0.63680800 & -0.26678300 \\
\hline 1435 & $\mathrm{H}$ & & -0.3 & 1687600 & 0.74819700 & -1.35577800 \\
\hline 1436 & $\mathrm{C}$ & & -0.99 & 370400 & -0.65507500 & 0.04854100 \\
\hline 1437 & $\mathrm{H}$ & & -2.0 & 1912300 & -0.72576100 & -0.44222000 \\
\hline 1438 & $\mathrm{O}$ & & -0.6 & 193800 & -1.52236400 & 0.77417200 \\
\hline 1439 & $\mathrm{O}$ & & -3.6 & 4600900 & -0.42009200 & -0.53278700 \\
\hline 1440 & $\mathrm{H}$ & & -3.85521 & -0.5 & 97891200 & 23384900 \\
\hline 1441 & Frequencies $[1$ & $/ \mathrm{cm}]$ & & & & \\
\hline 1442 & -413.12 & 230.97 & 838.37 & 1130.42 & $1344.98 \quad 2887.3$ & $32 \quad 2974.22$ \\
\hline 1443 & 18.32 & 250.91 & 849.61 & 1197.31 & $1404.41 \quad 2890.3$ & $33 \quad 2985.03$ \\
\hline 1444 & 54.27 & 294.31 & 869.66 & 1214.34 & $1418.74 \quad 2897.2$ & $26 \quad 3577.87$ \\
\hline 1445 & 72.47 & 373.13 & 908.83 & 1254.36 & $1424.27 \quad 2900.7$ & \\
\hline 1446 & 104.18 & 409.92 & 976.97 & 1266.93 & $1425.78 \quad 2904.0$ & \\
\hline 1447 & 105.28 & 565.36 & 1003.83 & 1297.26 & $1430.06 \quad 2927.5$ & \\
\hline 1448 & 158.23 & 617.62 & 1021.90 & 1320.35 & $\begin{array}{ll}1432.53 & 2944.7\end{array}$ & \\
\hline 1449 & 166.24 & 714.15 & 1074.19 & 1329.02 & 1768.112966 .9 & \\
\hline 1450 & 225.08 & 799.79 & 1090.68 & 1341.15 & $\begin{array}{c}2073.66 \quad 2973.7 \\
\text { S-34 }\end{array}$ & \\
\hline
\end{tabular}


P-abs2

1453

1454

1455

$\mathrm{C}$

$\mathrm{H}$

0.71994300

$$
\begin{array}{ccc}
0.71994300 & 0.64306600 & -0.00022700 \\
0.94143200 & 1.27981800 & 0.86643300 \\
0.94132500 & 1.27957000 & -0.86709300 \\
1.67118200 & -0.54875200 & -0.00007400 \\
1.48202300 & -1.17273500 & -0.87569400 \\
1.48169900 & -1.17267800 & 0.87552100 \\
3.12518000 & -0.09458500 & 0.00017800 \\
3.80670700 & -0.94375000 & 0.00018700 \\
3.34354700 & 0.51093900 & -0.88052300 \\
3.34330800 & 0.51076800 & 0.88105800 \\
-1.67098400 & 1.52560700 & 0.00009200 \\
-2.70551100 & 1.19469000 & -0.00022800 \\
-1.49243500 & 2.15502300 & 0.87634200 \\
-1.49198500 & 2.15554000 & -0.87569200 \\
-0.74531500 & 0.36340800 & -0.00005900 \\
-1.28841100 & -0.95991500 & -0.00006000 \\
-0.57051600 & -1.79269600 & -0.00022300 \\
-2.49364600 & -1.19718300 & 0.00010100
\end{array}
$$$$
\mathrm{H}
$$

1.67118200

1.48202300

1458

1.48169900

3.12518000

1460

3.80670700

3.34354700

3.34330800

1463

$-1.67098400$

1464

$-2.70551100$

$-1.49243500$

1466

$-1.49198500$

$-0.74531500$

$-1.28841100$

$1469 \quad \mathrm{H}$

$-0.57051600$

$-2.49364600$

1471 Frequencies[1/cm]

1472

1473

1474

1475

1476

1477

1478

1479

1480

1481

1482

1483

1484

1485

$56.39 \quad 392.24$

$\begin{array}{llll}1013.12 & 1343.37 & 1534.36 & 2966.84\end{array}$

$\begin{array}{lllllll}85.66 & 619.29 & 1050.68 & 1354.88 & 2849.65 & 2976.94\end{array}$

$\begin{array}{lllllll}100.41 & 694.92 & 1065.09 & 1371.58 & 2866.84 & 3008.80\end{array}$

$\begin{array}{llllll}160.17 & 785.01 & 1171.97 & 1380.29 & 2867.99\end{array}$

$\begin{array}{llllll}203.06 & 808.54 & 1198.59 & 1406.86 & 2883.68\end{array}$

$\begin{array}{llllll}236.62 & 874.03 & 1250.18 & 1418.33 & 2900.25\end{array}$

$\begin{array}{llllll}273.99 & 909.93 & 1258.56 & 1420.82 & 2906.12\end{array}$

$\begin{array}{llllll}341.99 & 943.53 & 1289.81 & 1426.68 & 2926.97\end{array}$

$363.73 \quad 949.78$

$\begin{array}{lll}1324.63 & 1435.07 & 2937.71\end{array}$

TS-abs2

$\mathrm{C}$
$\mathrm{H}$
$\mathrm{H}$
$\mathrm{C}$
$\mathrm{H}$
$\mathrm{H}$
$\mathrm{C}$
$\mathrm{H}$
$\mathrm{H}$
$\mathrm{H}$
$\mathrm{C}$
$\mathrm{H}$
$\mathrm{H}$
$\mathrm{H}$

$\begin{array}{ccc}0.84651700 & 0.83323500 & -0.07506700 \\ 1.06127600 & 1.52566400 & 0.74922000 \\ 1.12061600 & 1.35791700 & -0.99347300 \\ 1.68979800 & -0.42870100 & 0.07337400 \\ 1.42211500 & -1.12280100 & -0.72537000 \\ 1.43742400 & -0.93028700 & 1.01162700 \\ 3.18156600 & -0.12643600 & 0.03952700 \\ 3.77323900 & -1.03404200 & 0.14874300 \\ 3.46011000 & 0.34302100 & -0.90485500 \\ 3.46141500 & 0.55524600 & 0.84418400 \\ -1.49444100 & 1.79859500 & -0.37885100 \\ -2.54864000 & 1.54516100 & -0.48037400 \\ -1.38911200 & 2.51872800 & 0.43739300 \\ -1.16466200 & 2.28239500 & -1.29687700 \\ & \mathrm{~S}-35 & \end{array}$




\begin{tabular}{|c|c|c|c|c|c|}
\hline 1497 & $\mathrm{C}$ & & -0.65163600 & 0.56286600 & -0.10882600 \\
\hline 1498 & $\mathrm{H}$ & & -0.85475600 & -0.21541100 & -0.97155500 \\
\hline 1499 & $\mathrm{C}$ & & -1.10070000 & -0.11409100 & 1.15400700 \\
\hline 1500 & $\mathrm{H}$ & & -0.95675700 & 0.49214200 & 2.07346600 \\
\hline 1501 & $\mathrm{O}$ & & -1.58280500 & -1.21246800 & 1.22792800 \\
\hline 1502 & $\mathrm{O}$ & & -1.18654500 & -1.36693500 & -1.63751500 \\
\hline 1503 & $\mathrm{H}$ & & -1.49408700 & -1.83532300 & -0.84041900 \\
\hline 1504 & \multicolumn{2}{|c|}{ Frequencies $[1 / \mathrm{cm}]$} & & & \\
\hline 1505 & -821.87 & 274.20 & $841.90 \quad 1114.41$ & $1343.33 \quad 2763.2$ & $22 \quad 2976.45$ \\
\hline 1506 & 54.48 & 323.31 & $865.42 \quad 1152.62$ & $1400.33 \quad 2860.2$ & $21 \quad 2986.54$ \\
\hline 1507 & 65.18 & 339.19 & $909.34 \quad 1172.88$ & $1405.05 \quad 2890.9$ & $93 \quad 3532.92$ \\
\hline 1508 & 73.07 & 408.57 & $931.97 \quad 1216.34$ & $1415.63 \quad 2895.1$ & \\
\hline 1509 & 113.58 & 411.81 & $950.53 \quad 1253.24$ & $1419.12 \quad 2901.0$ & \\
\hline 1510 & 153.82 & 503.57 & $978.55 \quad 1269.56$ & $1426.18 \quad 2920.5$ & \\
\hline 1511 & 166.65 & 634.65 & $1012.13 \quad 1323.89$ & $1434.23 \quad 2939.4$ & \\
\hline 1512 & 193.59 & 701.92 & $1038.33 \quad 1325.83$ & $1436.56 \quad 2960.4$ & \\
\hline 1513 & 231.76 & 810.81 & $1084.63 \quad 1340.33$ & $1754.25 \quad 2965.3$ & \\
\hline \multicolumn{6}{|l|}{1514} \\
\hline 1515 & \multicolumn{5}{|l|}{ P-abs $2 \mathrm{~m}$} \\
\hline 1516 & \multicolumn{2}{|l|}{$\mathrm{C}$} & -1.49177500 & 0.89208200 & 0.29076000 \\
\hline 1517 & \multicolumn{2}{|l|}{$\mathrm{H}$} & -2.38479000 & 1.08321600 & 0.91537500 \\
\hline 1518 & \multicolumn{2}{|l|}{$\mathrm{C}$} & -0.93095500 & -0.52334300 & 0.39551200 \\
\hline 1519 & \multicolumn{2}{|l|}{$\mathrm{H}$} & -0.87170000 & -0.76090100 & 1.46317100 \\
\hline 1520 & \multicolumn{2}{|l|}{$\mathrm{C}$} & 0.43968500 & -0.64359800 & -0.26156000 \\
\hline 1521 & \multicolumn{2}{|l|}{$\mathrm{H}$} & 0.36872100 & -0.25624900 & -1.28083000 \\
\hline 1522 & \multicolumn{2}{|l|}{$\mathrm{H}$} & 0.69835700 & -1.70245000 & -0.33688600 \\
\hline 1523 & \multicolumn{2}{|l|}{$\mathrm{C}$} & 1.53804000 & 0.09955200 & 0.48994700 \\
\hline 1524 & $\mathrm{H}$ & & 1.59880300 & -0.28688000 & 1.51110400 \\
\hline 1525 & $\mathrm{H}$ & & 1.27266600 & 1.15501500 & 0.56381900 \\
\hline 1526 & $\mathrm{C}$ & & 2.89155400 & -0.04232800 & -0.19393900 \\
\hline 1527 & $\mathrm{H}$ & & 3.67310700 & 0.48319300 & 0.35336300 \\
\hline 1528 & $\mathrm{H}$ & & 3.18199600 & -1.09132100 & -0.27014600 \\
\hline 1529 & $\mathrm{H}$ & & 2.85661800 & 0.36711200 & -1.20416800 \\
\hline 1530 & $\mathrm{C}$ & & -1.96285000 & -1.38928800 & -0.24397300 \\
\hline 1531 & $\mathrm{H}$ & & -1.95049000 & -1.51223600 & -1.31732400 \\
\hline 1532 & $\mathrm{H}$ & & -2.82560900 & -1.73701800 & 0.30292800 \\
\hline 1533 & $\mathrm{O}$ & & -1.06498400 & 1.73750800 & -0.44511200 \\
\hline 1534 & Frequencies[1 & /cm] & & & \\
\hline 1535 & 75.75 & 412.27 & $996.35 \quad 1293.29$ & $1752.68 \quad 2972.4$ & \\
\hline 1536 & 104.21 & 504.56 & $1025.37 \quad 1327.37$ & $2796.52 \quad 3004.3$ & \\
\hline 1537 & 112.17 & 617.15 & $1052.41 \quad 1341.52$ & $2884.32 \quad 3109.0$ & \\
\hline 1538 & 163.32 & 716.54 & $1096.71 \quad 1352.20$ & 2891.55 & \\
\hline 1539 & 173.55 & 792.47 & $1115.79 \quad 1386.16$ & 2898.70 & \\
\hline 1540 & 237.61 & 837.48 & $1185.84 \quad 1405.64$ & 2901.64 & \\
\hline 1541 & 263.56 & 855.07 & $1210.28 \quad 1419.72$ & 2932.54 & \\
\hline 1542 & 302.20 & 891.50 & $1241.57 \quad 1426.89$ & 2947.80 & \\
\hline
\end{tabular}




\begin{tabular}{|c|c|c|c|c|c|}
\hline \multirow{2}{*}{$\begin{array}{l}1543 \\
1544\end{array}$} & 375.10 & \multirow[t]{2}{*}{944.84} & \multirow[t]{2}{*}{$1266.40 \quad 1433.28$} & \multicolumn{2}{|l|}{2967.27} \\
\hline & & & & & \\
\hline 1545 & TS-abs2m & & \multirow[b]{2}{*}{0.76063900} & & \\
\hline 1546 & $\mathrm{C}$ & & & 1.14197300 & -0.15451900 \\
\hline 1547 & $\mathrm{H}$ & & 1.70104800 & 1.40776800 & -0.67074000 \\
\hline 1548 & $\mathrm{C}$ & & 0.38426600 & -0.32245800 & -0.23919900 \\
\hline 1549 & $\mathrm{H}$ & & 0.43099000 & -0.59887300 & -1.29834700 \\
\hline 1550 & $\mathrm{C}$ & & -1.01145700 & -0.59701100 & 0.31490200 \\
\hline 1551 & $\mathrm{H}$ & & -1.08226300 & -0.15386800 & 1.31113100 \\
\hline 1552 & $\mathrm{H}$ & & -1.14036200 & -1.67615600 & 0.43323500 \\
\hline 1553 & $\mathrm{C}$ & & -2.12937200 & -0.04835300 & -0.56574200 \\
\hline 1554 & $\mathrm{H}$ & & -2.05494600 & -0.49704600 & -1.55991000 \\
\hline 1555 & $\mathrm{H}$ & & -1.99348800 & 1.02657200 & -0.68940400 \\
\hline 1556 & $\mathrm{C}$ & & -3.50554800 & -0.32836000 & 0.02367600 \\
\hline 1557 & $\mathrm{H}$ & & -4.29926000 & 0.05621500 & -0.61557000 \\
\hline 1558 & $\mathrm{H}$ & & -3.66570000 & -1.40033800 & 0.14966900 \\
\hline 1559 & $\mathrm{H}$ & & -3.60726700 & 0.14065900 & 1.00298300 \\
\hline 1560 & $\mathrm{C}$ & & 1.45012500 & -1.11677900 & 0.50129000 \\
\hline 1561 & $\mathrm{H}$ & & 1.49317200 & -0.86928600 & 1.56171000 \\
\hline 1562 & $\mathrm{H}$ & & 1.34794200 & -2.19146400 & 0.36375100 \\
\hline 1563 & $\mathrm{H}$ & & 2.50393200 & -0.86413200 & 0.04389600 \\
\hline 1564 & $\mathrm{O}$ & & 3.69932200 & -0.32483200 & -0.41988800 \\
\hline 1565 & $\mathrm{H}$ & & 3.97825700 & 0.09763900 & 0.40812800 \\
\hline 1566 & $\mathrm{O}$ & & 0.13768100 & 1.96836100 & 0.45451500 \\
\hline 1567 & Frequencies $[1$ & $/ \mathrm{cm}]$ & & & \\
\hline 1568 & -788.68 & 254.96 & $839.36 \quad 1100.72$ & $1341.27 \quad 2822.7$ & $\begin{array}{ll}71 & 2973.36\end{array}$ \\
\hline 1569 & 38.72 & 295.13 & $853.33 \quad 1143.39$ & $1347.62 \quad 2886.4$ & $43 \quad 2998.47$ \\
\hline 1570 & 66.94 & 326.38 & $879.44 \quad 1200.41$ & $1361.47 \quad 2891.5$ & $59 \quad 3591.94$ \\
\hline 1571 & 70.91 & 378.60 & $913.22 \quad 1217.61$ & $1392.34 \quad 2897.5$ & \\
\hline 1572 & 107.45 & 416.48 & $981.89 \quad 1245.14$ & $1405.47 \quad 2900.4$ & \\
\hline 1573 & 112.34 & 615.83 & $994.49 \quad 1260.78$ & $1418.66 \quad 2930.5$ & \\
\hline 1574 & 151.41 & 712.26 & $1021.85 \quad 1268.88$ & $1426.60 \quad 2932.0$ & \\
\hline 1575 & 170.14 & 749.63 & $1048.43 \quad 1312.69$ & $1431.75 \quad 2947.5$ & \\
\hline 1576 & 234.84 & 801.85 & $1089.56 \quad 1324.99$ & $1761.03 \quad 2967.9$ & \\
\hline 1577 & & & & & \\
\hline 1578 & P-abs3 & & & & \\
\hline 1579 & $\mathrm{C}$ & & 0.28024300 & -0.90883600 & -0.08716000 \\
\hline 1580 & $\mathrm{H}$ & & 0.30532300 & -1.52106400 & -0.98009400 \\
\hline 1581 & $\mathrm{C}$ & & 1.56415100 & -0.56141700 & 0.58053100 \\
\hline 1582 & $\mathrm{H}$ & & 1.97891800 & -1.44672400 & 1.07843200 \\
\hline 1583 & $\mathrm{H}$ & & 1.38143500 & 0.17604800 & 1.36481200 \\
\hline 1584 & $\mathrm{C}$ & & 2.60301900 & -0.01564900 & -0.40239600 \\
\hline 1585 & $\mathrm{H}$ & & 3.54247500 & 0.20773500 & 0.10269900 \\
\hline 1586 & $\mathrm{H}$ & & 2.80706800 & -0.74505900 & -1.18718300 \\
\hline 1587 & $\mathrm{H}$ & & 2.22932800 & 0.89415900 & -0.86903600 \\
\hline 1588 & $\mathrm{C}$ & & -2.22525800 & $\begin{array}{c}-0.98553800 \\
S-37\end{array}$ & -0.35318700 \\
\hline
\end{tabular}




\begin{tabular}{|c|c|c|c|c|c|}
\hline 1589 & $\mathrm{H}$ & & -3.16485900 & -0.54643000 & -0.01758800 \\
\hline 1590 & $\mathrm{H}$ & & -2.14229600 & -0.83627800 & -1.43014800 \\
\hline 1591 & $\mathrm{H}$ & & -2.26019100 & -2.05595900 & -0.15909000 \\
\hline 1592 & $\mathrm{C}$ & & -1.02454000 & -0.35880000 & 0.36054800 \\
\hline 1593 & $\mathrm{H}$ & & -1.15034700 & -0.52356900 & 1.44289000 \\
\hline 1594 & $\mathrm{C}$ & & -1.11003800 & 1.15266300 & 0.21546700 \\
\hline 1595 & $\mathrm{H}$ & & -2.07231000 & 1.58641800 & 0.55822500 \\
\hline 1596 & $\mathrm{O}$ & & -0.24750100 & 1.85952300 & -0.22334200 \\
\hline 1597 & \multicolumn{5}{|c|}{ Frequencies $[1 / \mathrm{cm}]$} \\
\hline 1598 & 56.22 & 426.46 & $1007.23 \quad 1325.35$ & \multicolumn{2}{|l|}{$1763.01 \quad 2984.08$} \\
\hline 1599 & 69.28 & 430.34 & $1012.90 \quad 1331.12$ & \multicolumn{2}{|l|}{$2766.64 \quad 2987.01$} \\
\hline 1600 & 97.40 & 645.19 & $1059.45 \quad 1336.85$ & \multicolumn{2}{|l|}{$2816.31 \quad 3018.29$} \\
\hline 1601 & 166.96 & 712.26 & $1115.81 \quad 1371.79$ & \multicolumn{2}{|l|}{2850.31} \\
\hline 1602 & 216.27 & 792.98 & $1133.12 \quad 1402.84$ & \multicolumn{2}{|l|}{2903.50} \\
\hline 1603 & 229.34 & 860.00 & $1202.48 \quad 1420.27$ & \multicolumn{2}{|l|}{2905.47} \\
\hline 1604 & 236.55 & 866.27 & $1220.88 \quad 1421.00$ & \multicolumn{2}{|l|}{2923.24} \\
\hline 1605 & 307.49 & 911.85 & $1228.19 \quad 1429.74$ & \multicolumn{2}{|l|}{2968.38} \\
\hline 1606 & 324.17 & 985.72 & $1267.44 \quad 1432.37$ & \multicolumn{2}{|l|}{2972.39} \\
\hline \multicolumn{6}{|l|}{1607} \\
\hline 1608 & \multicolumn{5}{|l|}{ TS-abs3 } \\
\hline 1609 & $\mathrm{C}$ & & 0.42246800 & 0.65327800 & 0.32872500 \\
\hline 1610 & $\mathrm{H}$ & & 0.43112700 & -0.32264000 & 0.98264300 \\
\hline 1611 & $\mathrm{H}$ & & 0.65877400 & 1.42930000 & 1.06154000 \\
\hline 1612 & $\mathrm{C}$ & & 1.51854000 & 0.52361600 & -0.70936100 \\
\hline 1613 & $\mathrm{H}$ & & 1.54006900 & 1.43586700 & -1.31540800 \\
\hline 1614 & $\mathrm{H}$ & & 1.28251000 & -0.29844200 & -1.38621800 \\
\hline 1615 & $\mathrm{C}$ & & 2.88032400 & 0.29073700 & -0.06732400 \\
\hline 1616 & $\mathrm{H}$ & & 3.66209900 & 0.19119300 & -0.81881100 \\
\hline 1617 & $\mathrm{H}$ & & 3.14769900 & 1.11990800 & 0.58934000 \\
\hline 1618 & $\mathrm{H}$ & & 2.86053200 & -0.61914800 & 0.53254800 \\
\hline 1619 & $\mathrm{C}$ & & -2.00095500 & 1.03721900 & 0.94076800 \\
\hline 1620 & $\mathrm{H}$ & & -3.01857800 & 1.13636800 & 0.56219100 \\
\hline 1621 & $\mathrm{H}$ & & -1.95827000 & 0.18162400 & 1.61504800 \\
\hline 1622 & $\mathrm{H}$ & & -1.76025400 & 1.93595000 & 1.50604200 \\
\hline 1623 & $\mathrm{C}$ & & -0.98541500 & 0.84546000 & -0.19589600 \\
\hline 1624 & $\mathrm{H}$ & & -1.01767200 & 1.73379900 & -0.83952900 \\
\hline 1625 & $\mathrm{C}$ & & -1.49244500 & -0.29684100 & -1.03992500 \\
\hline 1626 & $\mathrm{H}$ & & -2.41772800 & -0.07461100 & -1.60547500 \\
\hline 1627 & $\mathrm{O}$ & & -1.00828900 & -1.39643400 & -1.09943300 \\
\hline 1628 & $\mathrm{O}$ & & 0.32603000 & -1.62722000 & 1.46818700 \\
\hline 1629 & $\mathrm{H}$ & & -0.00733900 & -1.98075700 & 0.62413800 \\
\hline 1630 & Frequencies [1 & $/ \mathrm{cm}]$ & & & \\
\hline 1631 & -805.77 & 253.98 & $868.00 \quad 1122.80$ & $1340.91 \quad 2796.04$ & $4 \quad 2979.47$ \\
\hline 1632 & 66.44 & 311.07 & $886.90 \quad 1148.22$ & $1359.85 \quad 2866.13$ & $3 \quad 2984.28$ \\
\hline 1633 & 78.53 & 380.87 & $896.82 \quad 1168.33$ & $1371.44 \quad 2877.87$ & $7 \quad 3537.00$ \\
\hline 1634 & 96.84 & 409.30 & $910.10 \quad 1205.73$ & $\begin{array}{c}1411.992902 .36 \\
\text { S-38 }\end{array}$ & \\
\hline
\end{tabular}




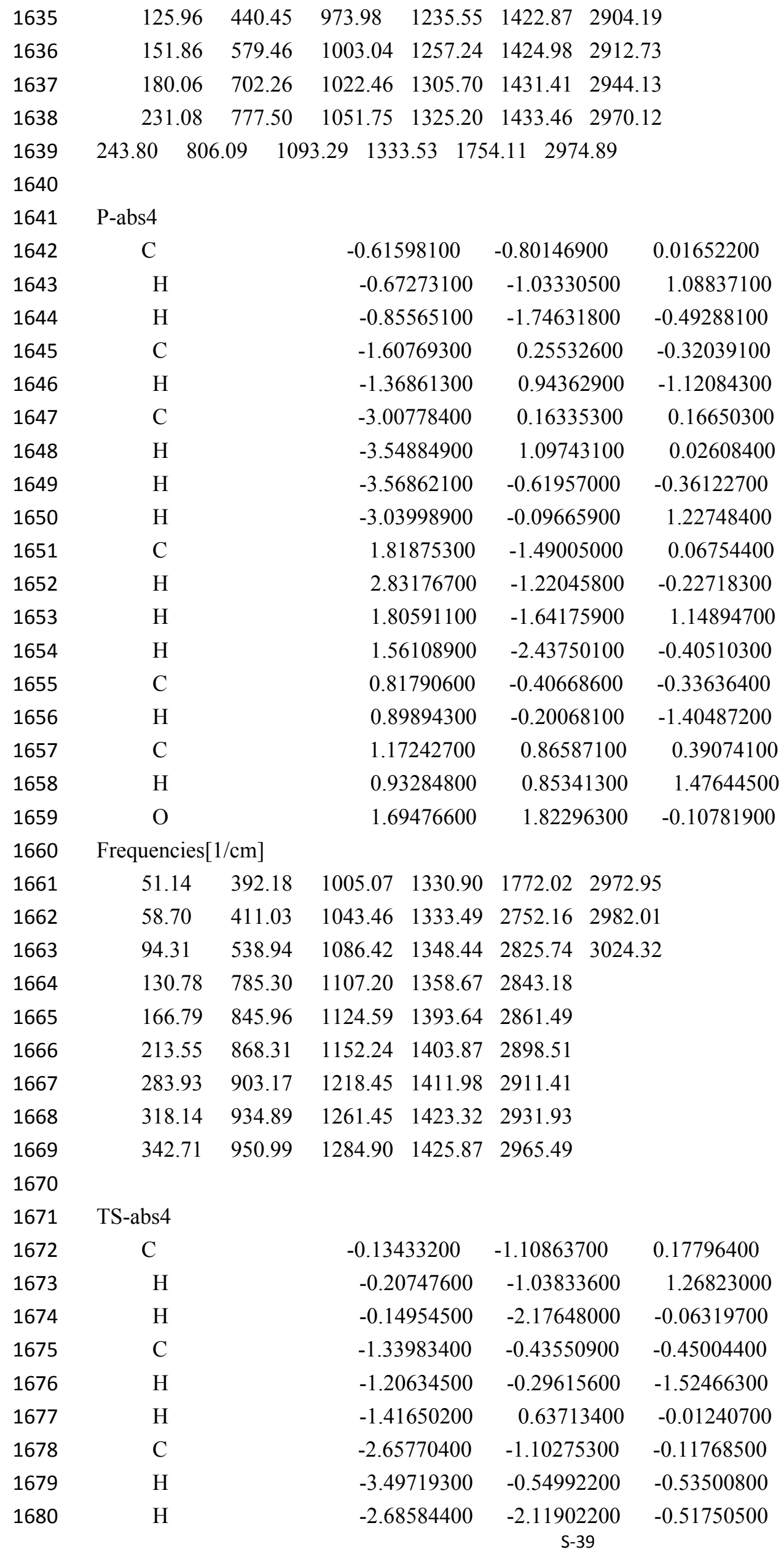




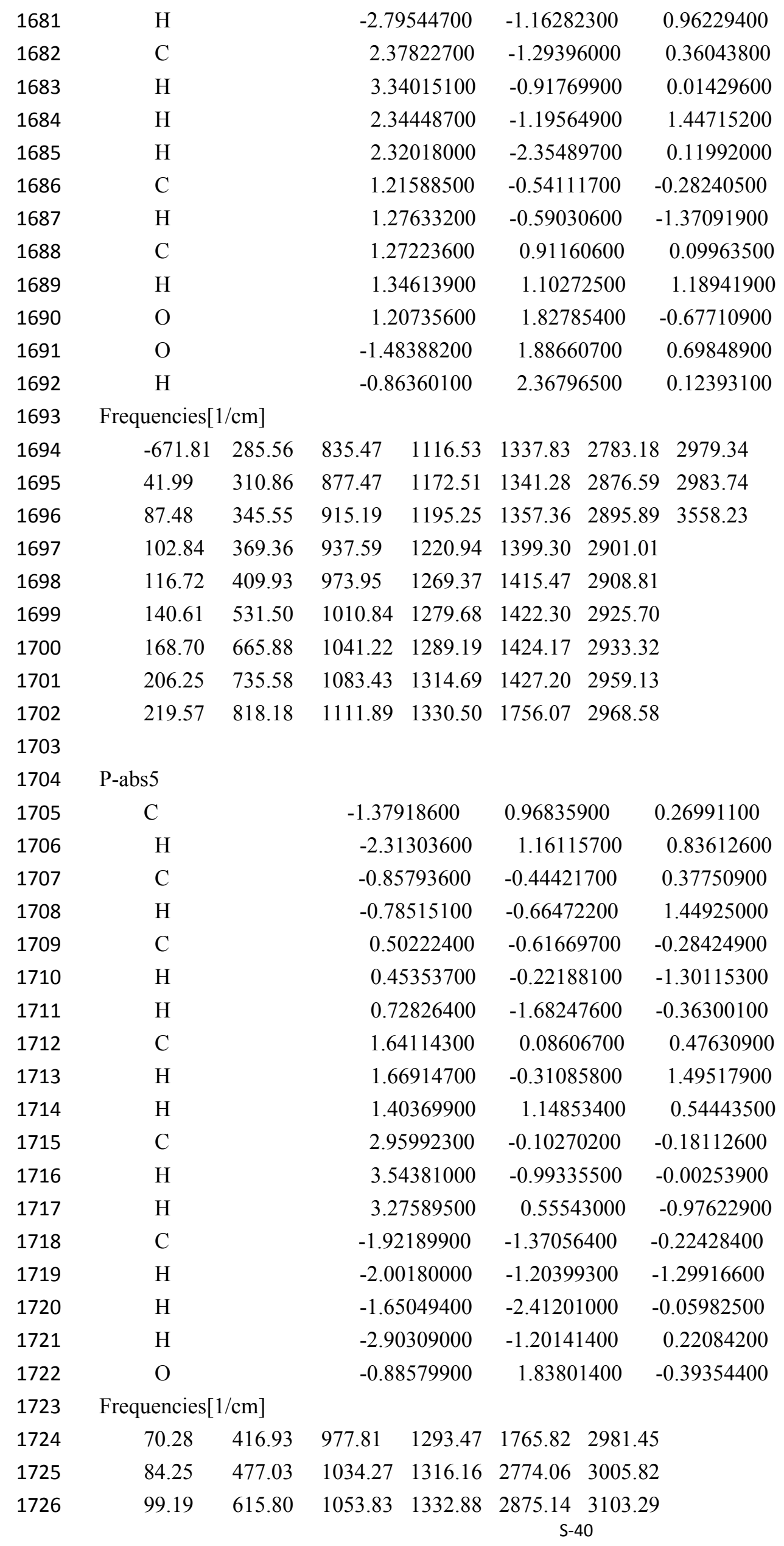




$\begin{array}{lccrrll}1727 & 121.26 & 711.06 & 1089.56 & 1356.67 & 2887.22 & \\ 1728 & 166.27 & 801.76 & 1119.09 & 1390.31 & 2901.59 & \\ 1729 & 219.65 & 825.16 & 1191.91 & 1405.65 & 2904.23 & \\ 1730 & 255.49 & 876.48 & 1204.30 & 1421.30 & 2937.92 & \\ 1731 & 317.25 & 894.74 & 1245.35 & 1424.09 & 2954.75 & \\ 1732 & 350.17 & 951.82 & 1261.55 & 1427.67 & 2968.18 & \\ 1733 & & & & & & \\ 1734 & \text { TS-abs } 5 & & & & \\ 1735 & \mathrm{C} & & 1.84159700 & 1.17464300 & -0.23033800 \\ 1736 & \mathrm{H} & & 2.64606700 & 1.60886500 & -0.85758100 \\ 1737 & \mathrm{C} & & 1.57869400 & -0.29264200 & -0.46898000 \\ 1738 & \mathrm{H} & & 1.40246300 & -0.40247300 & -1.54564100 \\ 1739 & \mathrm{C} & & 0.37434500 & -0.80095200 & 0.31134100 \\ 1740 & \mathrm{H} & & 0.49496300 & -0.51854100 & 1.36011200 \\ 1741 & \mathrm{H} & & 0.36381100 & -1.89321700 & 0.27301700 \\ 1742 & \mathrm{C} & & -0.95706600 & -0.26276300 & -0.21070400 \\ 1743 & \mathrm{H} & & -1.08653400 & -0.56127600 & -1.25362900 \\ 1744 & \mathrm{H} & & -0.93518900 & 0.82743700 & -0.18867500 \\ 1745 & \mathrm{C} & & -2.12543700 & -0.76632900 & 0.60658200 \\ 1746 & \mathrm{H} & & -2.23311200 & -1.85100200 & 0.58365400 \\ 1747 & \mathrm{H} & & -2.11062700 & -0.41381000 & 1.63709500 \\ 1748 & \mathrm{H} & & -3.11957600 & -0.37191100 & 0.13580300 \\ 1749 & \mathrm{C} & & 2.86485900 & -1.05145000 & -0.12030000 \\ 1750 & \mathrm{H} & & 3.05800400 & -0.99343700 & 0.95157100 \\ 1751 & \mathrm{H} & & 2.76840000 & -2.10097600 & -0.39322700 \\ 1752 & \mathrm{H} & & 3.72915200 & -0.64294300 & -0.64499400 \\ 1753 & \mathrm{O} & & -4.10931100 & 0.40449900 & -0.49375300 \\ 1754 & \mathrm{H} & & -3.82423800 & 1.25922000 & -0.13419600 \\ 1755 & \mathrm{O} & & 1.28236900 & 1.85187900 & 0.58763900\end{array}$

1756 Frequencies[1/cm]

$\begin{array}{llllllll}1757 & -757.58 & 250.45 & 846.15 & 1126.81 & 1333.75 & 2779.00 & 2980.86 \\ 1758 & 29.75 & 317.24 & 864.52 & 1190.68 & 1359.29 & 2876.31 & 2990.18 \\ 1759 & 51.21 & 325.98 & 880.09 & 1209.38 & 1388.46 & 2894.23 & 3596.14 \\ 1760 & 68.90 & 364.28 & 902.55 & 1231.98 & 1400.15 & 2902.34 & \\ 1761 & 76.48 & 418.36 & 974.32 & 1245.37 & 1412.26 & 2905.06 & \\ 1762 & 103.22 & 616.94 & 985.13 & 1263.82 & 1424.35 & 2924.99 & \\ 1763 & 135.96 & 705.23 & 1019.98 & 1280.67 & 1426.32 & 2932.60 & \\ 1764 & 165.17 & 744.50 & 1058.72 & 1310.40 & 1428.33 & 2954.17 & \\ 1765 & 220.59 & 796.77 & 1094.64 & 1327.29 & 1764.89 & 2968.68 & \\ 1766 & & & & & & & \\ 1767 & \text { PC-abs } 1 & & & & & & \\ 1768 & \mathrm{C} & & -0.72769900 & -0.19309600 & -0.38240900 \\ 1769 & \mathrm{H} & & -0.78941700 & -0.75626200 & -1.31740800 \\ 1770 & \mathrm{H} & & -0.47392500 & 0.83846500 & -0.63992600 \\ 1771 & \mathrm{C} & & -2.08350400 & -0.23315400 & 0.31454100 \\ 1772 & \mathrm{H} & & -2.06199200 & 0.40693900 & 1.19765800\end{array}$




\begin{tabular}{|c|c|c|c|c|c|}
\hline 1773 & \multicolumn{2}{|l|}{$\mathrm{H}$} & -2.28292400 & -1.24886700 & 0.66676200 \\
\hline 1774 & \multicolumn{2}{|l|}{$\mathrm{C}$} & -3.20282600 & 0.22251800 & -0.61336000 \\
\hline 1775 & \multicolumn{2}{|l|}{$\mathrm{H}$} & -3.27133400 & -0.42583400 & -1.48771900 \\
\hline 1776 & \multicolumn{2}{|l|}{$\mathrm{H}$} & -4.16823300 & 0.21010700 & -0.10923000 \\
\hline 1777 & \multicolumn{2}{|l|}{$\mathrm{H}$} & -3.02073400 & 1.23819100 & -0.96653000 \\
\hline 1778 & \multicolumn{2}{|l|}{$\mathrm{C}$} & 0.62169400 & -0.01774300 & 1.78000500 \\
\hline 1779 & \multicolumn{2}{|l|}{$\mathrm{H}$} & 0.73550600 & 1.04887300 & 1.58457700 \\
\hline 1780 & \multicolumn{2}{|l|}{$\mathrm{H}$} & -0.22899200 & -0.16402600 & 2.44304100 \\
\hline 1781 & \multicolumn{2}{|l|}{$\mathrm{H}$} & 1.51225000 & -0.37367900 & 2.29690700 \\
\hline 1782 & \multicolumn{2}{|l|}{$\mathrm{C}$} & 0.40430200 & -0.77532900 & 0.47489500 \\
\hline 1783 & \multicolumn{2}{|l|}{$\mathrm{H}$} & 0.21396900 & -1.83617100 & 0.66669400 \\
\hline 1784 & $\mathrm{C}$ & & 1.66862200 & -0.71816000 & -0.37374700 \\
\hline 1785 & $\mathrm{H}$ & & 1.95321500 & 1.56438300 & -0.43149000 \\
\hline 1786 & $\mathrm{O}$ & & 2.19964700 & -1.57604500 & -0.97040200 \\
\hline 1787 & $\mathrm{O}$ & & 1.54316100 & 2.42728200 & -0.29102700 \\
\hline 1788 & $\mathrm{H}$ & & 1.85661300 & 2.97777500 & -1.01146200 \\
\hline 1789 & Frequencies[ & $/ \mathrm{cm}]$ & & & \\
\hline 1790 & 34.59 & 238.79 & $773.81 \quad 1130.71$ & $1412.58 \quad 2889.9$ & $4 \quad 2988.95$ \\
\hline 1791 & 55.41 & 252.36 & $830.95 \quad 1181.75$ & $\begin{array}{ll}1422.03 & 2897.9\end{array}$ & $4 \quad 3578.38$ \\
\hline 1792 & 70.15 & 301.44 & $840.38 \quad 1218.65$ & $1425.90 \quad 2902.4$ & $17 \quad 3731.36$ \\
\hline 1793 & 86.39 & 324.87 & $895.00 \quad 1262.67$ & $1426.83 \quad 2909.6$ & \\
\hline 1794 & 103.50 & 350.70 & $968.54 \quad 1263.04$ & $\begin{array}{ll}1430.19 & 2929.1\end{array}$ & \\
\hline 1795 & 111.00 & 385.04 & $996.15 \quad 1294.51$ & $1436.07 \quad 2945.9$ & \\
\hline 1796 & 129.87 & 440.05 & $1028.52 \quad 1329.26$ & $1539.19 \quad 2968.5$ & \\
\hline 1797 & 177.48 & 519.67 & $1062.58 \quad 1341.14$ & $1883.09 \quad 2975.5$ & \\
\hline 1798 & 227.88 & 716.12 & $1071.94 \quad 1345.26$ & $2887.42 \quad 2979.1$ & \\
\hline 1799 & & & & & \\
\hline 1800 & PC-abs2 & & & & \\
\hline 1801 & $\mathrm{C}$ & & -1.47763000 & 1.20944500 & 0.24223700 \\
\hline 1802 & $\mathrm{H}$ & & -2.47377900 & 1.53838000 & 0.57876600 \\
\hline 1803 & $\mathrm{C}$ & & -1.36976600 & -0.14871600 & -0.17901600 \\
\hline 1804 & $\mathrm{H}$ & & 2.71684400 & 2.36333600 & -0.22529500 \\
\hline 1805 & $\mathrm{C}$ & & -0.05841800 & -0.69014800 & -0.61930100 \\
\hline 1806 & $\mathrm{H}$ & & 0.50259100 & 0.06663500 & -1.16927400 \\
\hline 1807 & $\mathrm{H}$ & & -0.21597600 & -1.54834800 & -1.27690800 \\
\hline 1808 & $\mathrm{C}$ & & 0.79430100 & -1.13201000 & 0.58613600 \\
\hline 1809 & $\mathrm{H}$ & & 0.23547800 & -1.86375900 & 1.17539000 \\
\hline 1810 & $\mathrm{H}$ & & 0.97036800 & -0.26295600 & 1.22065900 \\
\hline 1811 & $\mathrm{C}$ & & 2.12579500 & -1.71378200 & 0.13491500 \\
\hline 1812 & $\mathrm{H}$ & & 2.69102400 & -0.95622500 & -0.40602500 \\
\hline 1813 & $\mathrm{H}$ & & 2.71971200 & -2.03803100 & 0.98885300 \\
\hline 1814 & $\mathrm{H}$ & & 1.97590000 & -2.57542100 & -0.51790600 \\
\hline 1815 & $\mathrm{C}$ & & -2.54904800 & -1.04730500 & -0.11058400 \\
\hline 1816 & $\mathrm{H}$ & & -2.80786600 & -1.41105100 & -1.10917900 \\
\hline 1817 & $\mathrm{H}$ & & -2.31637500 & -1.93355700 & 0.48691500 \\
\hline 1818 & $\mathrm{H}$ & & -3.42098900 & $\begin{array}{c}-0.55579000 \\
\text { S-42 }\end{array}$ & 0.31786800 \\
\hline
\end{tabular}




\begin{tabular}{|c|c|c|c|c|c|c|}
\hline 1819 & \multicolumn{2}{|l|}{$\mathrm{O}$} & \multicolumn{2}{|c|}{2.20928500} & 1.55317800 & -0.28912500 \\
\hline 1820 & \multicolumn{2}{|l|}{$\mathrm{H}$} & \multicolumn{2}{|c|}{1.29327400} & 1.80413800 & -0.09379800 \\
\hline 1821 & \multicolumn{2}{|l|}{$\mathrm{O}$} & \multicolumn{2}{|c|}{-0.54198600} & 2.01029000 & 0.25207600 \\
\hline 1822 & \multicolumn{2}{|c|}{ Frequencies $[1 / \mathrm{cm}]$} & & & & \\
\hline 1823 & 34.20 & 241.12 & 796.61 & 1172.82 & $94.32 \quad 2878.22$ & 22985.30 \\
\hline 1824 & 98.80 & 269.24 & 848.05 & 1214.06 & $1407.59 \quad 2894.51$ & 113519.54 \\
\hline 1825 & 113.52 & 333.50 & 869.61 & 1242.70 & $1415.22 \quad 2900.24$ & $4 \quad 3737.43$ \\
\hline 1826 & 126.26 & 351.83 & 886.96 & 1265.13 & $1423.00 \quad 2903.5$ & \\
\hline 1827 & 131.55 & 386.54 & 950.66 & 1294.62 & 1429.192922 .79 & \\
\hline 1828 & 145.41 & 478.03 & 969.70 & 1315.09 & $1436.25 \quad 2945.76$ & \\
\hline 1829 & 148.00 & 557.59 & 1004.27 & 1336.79 & $1545.77 \quad 2963.20$ & \\
\hline 1830 & 173.15 & 583.20 & 1035.60 & 1341.95 & $\begin{array}{ll}1566.07 \quad 2970.12\end{array}$ & \\
\hline 1831 & \multirow[t]{2}{*}{228.55} & 716.83 & 1048.84 & 1372.18 & $2844.74 \quad 2977.94$ & \\
\hline \multicolumn{6}{|l|}{1832} & \\
\hline 1833 & \multicolumn{4}{|l|}{ PC-abs2m } & & \\
\hline 1834 & \multicolumn{2}{|l|}{$\mathrm{C}$} & \multicolumn{2}{|c|}{1.78513500} & -0.68950600 & -0.30835500 \\
\hline 1835 & \multicolumn{2}{|l|}{$\mathrm{H}$} & 2.2 & 0053200 & 0.29650000 & -0.59247600 \\
\hline 1836 & $\mathrm{C}$ & & 0.5 & 6221400 & -0.63301100 & 0.57812700 \\
\hline 1837 & $\mathrm{H}$ & & 0.4 & 3956100 & -1.62989500 & 1.02024100 \\
\hline 1838 & $\mathrm{C}$ & & -0.6 & 5876900 & -0.38028200 & -0.33676800 \\
\hline 1839 & $\mathrm{H}$ & & -0.7 & 0552400 & -1.19980800 & -1.05797600 \\
\hline 1840 & $\mathrm{H}$ & & -0.4 & 9392600 & 0.54089400 & -0.90188500 \\
\hline 1841 & $\mathrm{C}$ & & -1.97 & 7903700 & -0.29356700 & 0.41847200 \\
\hline 1842 & $\mathrm{H}$ & & -1.9 & 6762600 & 0.57338100 & 1.08253100 \\
\hline 1843 & $\mathrm{H}$ & & -2.0 & 9176600 & -1.17488800 & 1.05581300 \\
\hline 1844 & $\mathrm{C}$ & & -3.16 & 6558200 & -0.18575700 & -0.53132800 \\
\hline 1845 & $\mathrm{H}$ & & -3.2 & 2261200 & -1.05969000 & -1.18109300 \\
\hline 1846 & $\mathrm{H}$ & & -4.1 & 671700 & -0.10861100 & 0.01176800 \\
\hline 1847 & $\mathrm{H}$ & & $-3.0^{\circ}$ & 7086200 & 0.69457700 & -1.16834400 \\
\hline 1848 & $\mathrm{C}$ & & 0.7 & 2532500 & 0.38851800 & 1.64879500 \\
\hline 1849 & $\mathrm{H}$ & & 0.6 & 7371500 & 2.40200800 & 0.26233600 \\
\hline 1850 & $\mathrm{H}$ & & -0.0 & 5001000 & 0.53021300 & 2.38656400 \\
\hline 1851 & $\mathrm{H}$ & & 1.6 & 9989800 & 0.80423900 & 1.86595500 \\
\hline 1852 & $\mathrm{O}$ & & 0.9 & 9759400 & 2.53680900 & -0.63396500 \\
\hline 1853 & $\mathrm{H}$ & & 1.0 & 0316600 & 3.48650200 & -0.77042400 \\
\hline 1854 & $\mathrm{O}$ & & & 6196300 & -1.71103300 & -0.71936900 \\
\hline 1855 & Frequencies[ & $/ \mathrm{cm}]$ & & & & \\
\hline 1856 & 23.06 & 184.84 & 716.65 & 1097.95 & $1358.09 \quad 2856.58$ & $8 \quad 3094.11$ \\
\hline 1857 & 49.35 & 236.88 & 815.50 & 1108.65 & $1394.42 \quad 2888.55$ & $5 \quad 3634.38$ \\
\hline 1858 & 57.58 & 273.61 & 836.03 & 1179.27 & $1415.30 \quad 2898.80$ & $\begin{array}{l}30 \\
3745.77\end{array}$ \\
\hline 1859 & 74.89 & 284.41 & 874.99 & 1201.35 & $1423.02 \quad 2901.83$ & \\
\hline 1860 & 86.14 & 317.65 & 918.45 & 1247.91 & $1426.23 \quad 2921.27$ & \\
\hline 1861 & 108.08 & 363.41 & 956.84 & 1264.84 & $1435.01 \quad 2944.27$ & \\
\hline 1862 & 124.97 & 395.88 & 1012.03 & 1275.46 & $1525.97 \quad 2967.15$ & \\
\hline 1863 & 145.64 & 532.23 & 1043.47 & 1325.90 & 1767.892972 .99 & \\
\hline 1864 & 176.23 & 580.52 & 1055.50 & 1341.75 & $\begin{array}{c}2811.27 \quad 2996.41 \\
\text { S-43 }\end{array}$ & \\
\hline
\end{tabular}


PC-abs3

186

1868

1869

1870

1871

1872

1873

1874

1875

1876

1877

1878

1879

1880

1881

1882

1883

1884

1885

1886

1887

1888

1889

1890

1891

1892

1893

1894

1895

1896

1897

1898

1899

1900

1901

1902

1903

1904

1905

1906

1907

1908

1909

1910

PC-abs4

C

$\mathrm{H}$

C

$\mathrm{H}$

C

$\mathrm{H}$

$\mathrm{H}$

$\mathrm{C}$

$\mathrm{H}$

$\mathrm{H}$

C

$\begin{array}{ccc}2.20661500 & 0.04762400 & -0.51578200 \\ 2.00894500 & 0.77631600 & -1.33077300 \\ 1.06981600 & -0.11674200 & 0.45779200 \\ 1.40310700 & -0.81185900 & 1.23016400 \\ -0.12543000 & -0.71500100 & -0.29673000 \\ 0.18697700 & -1.66165800 & -0.76184000 \\ -0.40399000 & -0.05209700 & -1.12517800 \\ -1.31876500 & -0.95770000 & 0.56172500 \\ -2.00704400 & 1.27612600 & 0.07605400 \\ -1.15716500 & -1.15669500 & 1.61452200 \\ -2.59198000 & -1.40686400 & -0.06464900 \\ & \mathrm{~S}-44 & \end{array}$

$\begin{array}{ccc}1.95627800 & 0.27644100 & 0.23556500 \\ 2.98918200 & -0.01339900 & -0.03781200 \\ 0.87027600 & -0.61216900 & -0.32273300 \\ 0.74411100 & -0.26233500 & -1.35758200 \\ -0.42245400 & -0.45312800 & 0.39640200 \\ -1.22245800 & 2.25201700 & -0.60022700 \\ -0.40061300 & -0.28638200 & 1.46593900 \\ -1.71725700 & -0.72387600 & -0.28244100 \\ -1.83266500 & -1.79919100 & -0.47862400 \\ -1.70335800 & -0.24835100 & -1.26944600 \\ -2.91800000 & -0.23427100 & 0.52003800 \\ -2.85237600 & 0.84010600 & 0.69909400 \\ -3.85409700 & -0.43490000 & 0.00144700 \\ -2.95771700 & -0.72558700 & 1.49251900 \\ 1.36278900 & -2.06892600 & -0.37114600 \\ 1.51803600 & -2.45467000 & 0.63679900 \\ 0.62533700 & -2.69892600 & -0.86472000 \\ 2.30114600 & -2.15024300 & -0.92188700 \\ -0.31131300 & 2.34070000 & -0.88663700 \\ 0.20929500 & 2.17912500 & -0.08989200 \\ 1.76711100 & 1.24658900 & 0.92042200\end{array}$

encies[1/cm]

$\begin{array}{lllllll}5.27 & 218.13 & 732.93 & 1099.91 & 1367.38 & 2830.57 & 3023.00\end{array}$

$\begin{array}{lllllll}39.86 & 241.96 & 784.05 & 1125.50 & 1401.22 & 2852.88 & 3608.27\end{array}$

$\begin{array}{lllllll}59.83 & 252.04 & 865.89 & 1191.66 & 1420.73 & 2881.63 & 3734.48\end{array}$

$\begin{array}{lllllll}78.43 & 271.10 & 886.73 & 1209.80 & 1421.10 & 2900.51\end{array}$

$\begin{array}{llllll}111.28 & 326.90 & 917.32 & 1214.32 & 1427.12 & 2902.10\end{array}$

$\begin{array}{llllll}123.03 & 415.11 & 993.73 & 1290.14 & 1428.92 & 2963.13\end{array}$

$\begin{array}{lllllll}141.16 & 444.58 & 1004.87 & 1326.67 & 1549.26 & 2967.08\end{array}$

$\begin{array}{lllllll}167.15 & 488.32 & 1024.11 & 1340.25 & 1751.59 & 2979.51\end{array}$

$\begin{array}{llllll}07.16 & 613.35 & 1066.38 & 1349.33 & 2799.67 & 2984.96\end{array}$ 


\begin{tabular}{|c|c|c|c|c|c|}
\hline 1911 & \multicolumn{2}{|l|}{$\mathrm{H}$} & -2.51145600 & -2.43911400 & -0.42969300 \\
\hline 1912 & \multicolumn{2}{|l|}{$\mathrm{H}$} & -3.42483400 & -1.37873500 & 0.63683100 \\
\hline 1913 & \multicolumn{2}{|l|}{$\mathrm{H}$} & -2.83997200 & -0.78610800 & -0.92852700 \\
\hline 1914 & \multicolumn{2}{|l|}{$\mathrm{C}$} & 0.73643000 & 1.24660800 & 1.06488300 \\
\hline 1915 & \multicolumn{2}{|l|}{$\mathrm{H}$} & 0.39894300 & 1.93877200 & 0.29125800 \\
\hline 1916 & \multicolumn{2}{|l|}{$\mathrm{H}$} & -0.06499300 & 1.15284700 & 1.79766400 \\
\hline 1917 & \multicolumn{2}{|l|}{$\mathrm{H}$} & 1.60300800 & 1.67569200 & 1.56708600 \\
\hline 1918 & \multicolumn{2}{|l|}{$\mathrm{O}$} & -2.02982000 & 1.85100300 & -0.69710400 \\
\hline 1919 & \multicolumn{2}{|l|}{$\mathrm{H}$} & -2.83026900 & 2.37183300 & -0.60641900 \\
\hline 1920 & \multicolumn{2}{|l|}{$\mathrm{O}$} & 3.25214900 & -0.53761200 & -0.46221900 \\
\hline 1921 & \multicolumn{2}{|c|}{ Frequencies[1/cm] } & & & \\
\hline 1922 & 40.19 & 220.50 & $801.74 \quad 1101.65$ & $1356.02 \quad 2825.53$ & $3 \quad 3009.51$ \\
\hline 1923 & 45.88 & 240.82 & $846.43 \quad 1123.61$ & $1395.78 \quad 2847.17$ & $7 \quad 3619.48$ \\
\hline 1924 & 68.18 & 290.92 & $861.68 \quad 1180.17$ & $1406.88 \quad 2879.68$ & $8 \quad 3740.48$ \\
\hline 1925 & 93.54 & 330.25 & $901.13 \quad 1206.43$ & $1415.01 \quad 2900.51$ & \\
\hline 1926 & 107.20 & 333.85 & $939.59 \quad 1265.68$ & $1425.37 \quad 2918.78$ & \\
\hline 1927 & 124.68 & 361.09 & $950.40 \quad 1284.91$ & $1427.80 \quad 2929.88$ & \\
\hline 1928 & 135.63 & 383.74 & $1020.81 \quad 1331.99$ & $1536.01 \quad 2968.81$ & \\
\hline 1929 & 148.60 & 469.46 & $1048.84 \quad 1338.25$ & $1771.61 \quad 2970.50$ & \\
\hline 1930 & 194.21 & 554.87 & $1086.43 \quad 1348.70$ & $2759.83 \quad 2974.29$ & \\
\hline 1931 & & & & & \\
\hline 1932 & PC-abs5 & & & & \\
\hline 1933 & $\mathrm{C}$ & & 2.18903200 & 0.15447600 & -0.54477300 \\
\hline 1934 & $\mathrm{H}$ & & 1.98330300 & 1.00691300 & -1.22733000 \\
\hline 1935 & $\mathrm{C}$ & & 1.11649200 & -0.09044700 & 0.48269100 \\
\hline 1936 & $\mathrm{H}$ & & 1.50586400 & -0.83060900 & 1.18416700 \\
\hline 1937 & $\mathrm{C}$ & & -0.10085800 & -0.66099900 & -0.25676200 \\
\hline 1938 & $\mathrm{H}$ & & 0.18549200 & -1.59301200 & -0.75074800 \\
\hline 1939 & $\mathrm{H}$ & & -0.40027500 & 0.04473000 & -1.03694400 \\
\hline 1940 & $\mathrm{C}$ & & -1.29972200 & -0.92286000 & 0.65135100 \\
\hline 1941 & $\mathrm{H}$ & & -1.59662500 & -0.00010000 & 1.15473400 \\
\hline 1942 & $\mathrm{H}$ & & -0.99889900 & -1.61695500 & 1.44977300 \\
\hline 1943 & $\mathrm{C}$ & & -2.46422500 & -1.47461100 & -0.09043300 \\
\hline 1944 & $\mathrm{H}$ & & -2.30992300 & -2.06964800 & -0.98014000 \\
\hline 1945 & $\mathrm{H}$ & & -3.44081000 & -1.51052900 & 0.37044300 \\
\hline 1946 & $\mathrm{H}$ & & -2.67499900 & 0.79887400 & -0.80894200 \\
\hline 1947 & $\mathrm{C}$ & & 0.79286100 & 1.22260300 & 1.19778900 \\
\hline 1948 & $\mathrm{H}$ & & 0.26441200 & 1.90250300 & 0.52682500 \\
\hline 1949 & $\mathrm{H}$ & & 0.15349400 & 1.04748800 & 2.06088000 \\
\hline 1950 & $\mathrm{H}$ & & 1.70042000 & 1.71095000 & 1.55306400 \\
\hline 1951 & $\mathrm{O}$ & & -2.30465800 & 1.68143400 & -0.69371800 \\
\hline 1952 & $\mathrm{H}$ & & -2.84890300 & 2.25954000 & -1.23197300 \\
\hline 1953 & $\mathrm{O}$ & & 3.18915500 & -0.49632400 & -0.66915500 \\
\hline 1954 & Frequencies[ & $1 / \mathrm{cm}]$ & & & \\
\hline 1955 & 37.82 & 219.28 & $719.25 \quad 1094.30$ & $1354.07 \quad 2828.14$ & $4 \quad 3091.01$ \\
\hline 1956 & 41.95 & 249.64 & $816.21 \quad 1118.37$ & $\begin{array}{c}1391.262891 .65 \\
\text { S- } 45\end{array}$ & $5 \quad 3615.45$ \\
\hline
\end{tabular}




\begin{tabular}{|c|c|c|c|c|c|c|c|}
\hline 1957 & 50.57 & 294.52 & 860.09 & 1154.75 & 1396.09 & \multirow{2}{*}{$\begin{array}{l}2901.38 \\
2917.80\end{array}$} & $8 \quad 3739.25$ \\
\hline 1958 & 110.50 & 305.16 & 889.43 & 1214.32 & 1412.40 & & \\
\hline 1959 & 127.97 & 329.23 & 912.13 & 1246.25 & 1420.98 & 2923.85 & \\
\hline 1960 & 131.10 & 365.89 & 951.36 & 1268.70 & 1434.21 & 2940.09 & \\
\hline 1961 & 140.43 & 387.97 & 1013.83 & 1295.88 & 1537.67 & 2969.06 & \\
\hline 1962 & 163.59 & 523.19 & 1045.20 & 1335.57 & 1770.27 & 2982.81 & \\
\hline 1963 & 176.57 & 555.69 & 1061.75 & 1340.93 & 2758.80 & \multirow[t]{2}{*}{2994.37} & \\
\hline 1964 & & & & & & & \\
\hline 1965 & \multicolumn{2}{|l|}{ P-add } & & & & & \\
\hline 1966 & $\mathrm{C}$ & & \multicolumn{2}{|c|}{0.92714200} & \multicolumn{2}{|c|}{0.63583700} & 0.28186500 \\
\hline 1967 & $\mathrm{H}$ & & \multicolumn{2}{|c|}{0.92027700} & \multicolumn{2}{|c|}{0.32878500} & 1.33314800 \\
\hline 1968 & $\mathrm{H}$ & & \multicolumn{2}{|c|}{1.29008700} & \multicolumn{2}{|c|}{1.66753800} & 0.27066700 \\
\hline 1969 & $\mathrm{C}$ & & \multicolumn{2}{|c|}{1.90638500} & \multicolumn{2}{|c|}{-0.23403600} & -0.49825900 \\
\hline 1970 & $\mathrm{H}$ & & \multicolumn{2}{|c|}{1.94911900} & \multicolumn{2}{|c|}{0.12387400} & -1.53020400 \\
\hline 1971 & $\mathrm{H}$ & & \multicolumn{2}{|c|}{1.53319800} & \multicolumn{2}{|c|}{-1.25813200} & -0.53530000 \\
\hline 1972 & $\mathrm{C}$ & & \multicolumn{2}{|c|}{3.30214600} & \multicolumn{2}{|c|}{-0.21296100} & 0.11122800 \\
\hline 1973 & $\mathrm{H}$ & & \multicolumn{2}{|c|}{3.99942800} & -0.8188 & 5100 & -0.46600000 \\
\hline 1974 & $\mathrm{H}$ & & 3.69 & 575900 & 0.8040 & 8300 & 0.15180000 \\
\hline 1975 & $\mathrm{H}$ & & 3.28 & 3480200 & -0.6024 & 9000 & 1.13003000 \\
\hline 1976 & $\mathrm{C}$ & & -1.25 & 578900 & 1.9084 & 1500 & 0.06362000 \\
\hline 1977 & $\mathrm{H}$ & & -2.28 & 941200 & 1.8542 & 7000 & -0.26985000 \\
\hline 1978 & $\mathrm{H}$ & & -1.24 & 660000 & 2.0946 & 9700 & 1.13990000 \\
\hline 1979 & $\mathrm{H}$ & & -0.77 & 356000 & 2.7538 & 5600 & -0.42648300 \\
\hline 1980 & $\mathrm{C}$ & & -0.50 & 078200 & 0.6247 & 2200 & -0.25719200 \\
\hline 1981 & $\mathrm{H}$ & & -0.49 & 146500 & 0.4685 & 2000 & -1.33858700 \\
\hline 1982 & $\mathrm{C}$ & & -1.31 & 562100 & -0.57947 & 7100 & 0.31392300 \\
\hline 1983 & $\mathrm{H}$ & & -1.40 & 915200 & -0.38545 & 5500 & 1.40597200 \\
\hline 1984 & $\mathrm{O}$ & & -0.65 & 720200 & -1.72693 & 3600 & 0.20442200 \\
\hline 1985 & $\mathrm{O}$ & & -2.58 & 723800 & -0.57305 & 5600 & -0.28382100 \\
\hline 1986 & $\mathrm{H}$ & & -2.88 & 784300 & $-1.4857 \mathrm{~S}$ & 9400 & -0.32101700 \\
\hline 1987 & quencies & /cm] & & & & & \\
\hline 1988 & 59.42 & 323.88 & 886.52 & 1134.21 & 1338.81 & 2878.71 & 112972.76 \\
\hline 1989 & 72.09 & 350.87 & 918.53 & 1181.23 & 1340.65 & 2896.80 & $0 \quad 2996.78$ \\
\hline 1990 & 105.41 & 427.08 & 969.55 & 1220.46 & 1404.98 & 2897.83 & $3 \quad 3676.28$ \\
\hline 1991 & 126.86 & 489.22 & 1004.44 & 1227.75 & 1418.50 & 2900.00 & \\
\hline 1992 & 177.69 & 572.08 & 1010.11 & 1234.43 & 1418.90 & 2910.67 & \\
\hline 1993 & 228.32 & 686.90 & 1034.27 & 1254.19 & 1426.01 & 2918.5 & \\
\hline 1994 & 234.77 & 737.74 & 1080.68 & 1278.24 & 1429.84 & 2942.9 & \\
\hline 1995 & 241.53 & 833.81 & 1101.03 & 1292.78 & 1432.77 & 2964.5 & \\
\hline 1996 & 312.72 & 843.05 & 1115.26 & 1333.08 & 2691.77 & 2965.5 & \\
\hline 1997 & & & & & & & \\
\hline 1998 & -add & & & & & & \\
\hline 1999 & $\mathrm{C}$ & & 1.038 & 17100 & 0.71540 & 600 & 0.14252900 \\
\hline 2000 & $\mathrm{H}$ & & 1.09 & 489000 & 0.7893 & 36300 & 1.23508800 \\
\hline 2001 & $\mathrm{H}$ & & 1.43 & 882100 & 1.6573 & 32800 & -0.24179700 \\
\hline 2002 & $\mathrm{C}$ & & 1.91 & 123100 & $\begin{array}{r}-0.4358 \\
\text { s- } 4\end{array}$ & $\begin{array}{l}7900 \\
46\end{array}$ & -0.34092100 \\
\hline
\end{tabular}




\begin{tabular}{|c|c|c|c|c|c|}
\hline 2003 & $\mathrm{H}$ & & 1.84920400 & -0.49514300 & -1.43006600 \\
\hline 2004 & $\mathrm{H}$ & & 1.52241000 & -1.38302000 & 0.03592900 \\
\hline 2005 & $\mathrm{C}$ & & 3.36308000 & -0.26327800 & 0.08788700 \\
\hline 2006 & $\mathrm{H}$ & & 3.98346400 & -1.08537000 & -0.26653900 \\
\hline 2007 & $\mathrm{H}$ & & 3.77951900 & 0.66519400 & -0.30586400 \\
\hline 2008 & $\mathrm{H}$ & & 3.44546100 & -0.22730500 & 1.17525600 \\
\hline 2009 & $\mathrm{C}$ & & -1.17007100 & 1.92871200 & 0.04206500 \\
\hline 2010 & $\mathrm{H}$ & & -2.21198000 & 1.88350700 & -0.26617900 \\
\hline 2011 & $\mathrm{H}$ & & -1.13813700 & 2.12967200 & 1.11539400 \\
\hline 2012 & $\mathrm{H}$ & & -0.69220000 & 2.76647800 & -0.46435700 \\
\hline 2013 & $\mathrm{C}$ & & -0.43871400 & 0.63255800 & -0.27926700 \\
\hline 2014 & $\mathrm{H}$ & & -0.49772200 & 0.40449800 & -1.34499600 \\
\hline 2015 & $\mathrm{C}$ & & -1.04960000 & -0.53271900 & 0.45509600 \\
\hline 2016 & $\mathrm{H}$ & & -1.31718400 & -0.32775900 & 1.50478900 \\
\hline 2017 & $\mathrm{O}$ & & -0.93067400 & -1.70764000 & 0.07490800 \\
\hline 2018 & $\mathrm{O}$ & & -2.82802700 & -0.49611100 & -0.23578400 \\
\hline 2019 & $\mathrm{H}$ & & -3.11151500 & -1.41624100 & -0.10397000 \\
\hline 2020 & \multicolumn{2}{|c|}{ Frequencies $[1 / \mathrm{cm}]$} & & & \\
\hline 2021 & -535.81 & 265.75 & $835.90 \quad 1123.44$ & $1342.73 \quad 2868.65$ & $65 \quad 2972.64$ \\
\hline 2022 & 53.74 & 326.06 & $870.66 \quad 1182.42$ & $1407.21 \quad 2898.05$ & $5 \quad 2995.66$ \\
\hline 2023 & 62.80 & 358.58 & $917.19 \quad 1226.66$ & $1418.52 \quad 2900.8$ & $34 \quad 3576.20$ \\
\hline 2024 & 107.22 & 378.63 & $955.12 \quad 1252.71$ & $1421.57 \quad 2904.4 C$ & \\
\hline 2025 & 157.35 & 469.17 & $979.21 \quad 1276.81$ & $1426.37 \quad 2911.11$ & \\
\hline 2026 & 181.13 & 522.65 & $1010.54 \quad 1279.00$ & $1428.48 \quad 2932.21$ & \\
\hline 2027 & 198.77 & 702.18 & $1029.11 \quad 1321.97$ & $1434.94 \quad 2941.22$ & \\
\hline 2028 & 219.26 & 789.06 & $1076.97 \quad 1331.78$ & $1509.35 \quad 2964.61$ & \\
\hline 2029 & 234.90 & 810.41 & $1113.03 \quad 1340.60$ & $2830.83 \quad 2966.78$ & \\
\hline \multicolumn{6}{|l|}{2030} \\
\hline 2031 & TSdiss1 & & & & \\
\hline 2032 & $\mathrm{C}$ & & 0.89339700 & -0.34921500 & 0.15149200 \\
\hline 2033 & $\mathrm{H}$ & & 0.86203000 & -1.36454900 & -0.24559400 \\
\hline 2034 & $\mathrm{H}$ & & 0.87800500 & -0.44692400 & 1.24285700 \\
\hline 2035 & $\mathrm{C}$ & & 2.18886700 & 0.32866400 & -0.27888200 \\
\hline 2036 & $\mathrm{H}$ & & 2.27806900 & 1.30570700 & 0.19935500 \\
\hline 2037 & $\mathrm{H}$ & & 2.16010700 & 0.51203900 & -1.35683000 \\
\hline 2038 & $\mathrm{C}$ & & 3.40984900 & -0.51470300 & 0.06662500 \\
\hline 2039 & $\mathrm{H}$ & & 3.36592300 & -1.48336600 & -0.43239900 \\
\hline 2040 & $\mathrm{H}$ & & 4.33437500 & -0.02269900 & -0.23294100 \\
\hline 2041 & $\mathrm{H}$ & & 3.46044700 & -0.69880000 & 1.14069700 \\
\hline 2042 & $\mathrm{C}$ & & -0.46275000 & 1.80860200 & 0.25717500 \\
\hline 2043 & $\mathrm{H}$ & & -0.37653900 & 1.79792200 & 1.34647000 \\
\hline 2044 & $\mathrm{H}$ & & 0.33988600 & 2.42785200 & -0.13828700 \\
\hline 2045 & $\mathrm{H}$ & & -1.41167300 & 2.26862600 & -0.00697300 \\
\hline 2046 & $\mathrm{C}$ & & -0.36333800 & 0.39059500 & -0.29312500 \\
\hline 2047 & $\mathrm{H}$ & & -0.37351000 & 0.44175600 & -1.38873700 \\
\hline 2048 & $\mathrm{C}$ & & -1.60444900 & $\begin{array}{c}-0.44622500 \\
\text { S-47 }\end{array}$ & 0.02064200 \\
\hline
\end{tabular}




\begin{tabular}{|c|c|c|c|c|c|c|}
\hline 2049 & \multicolumn{2}{|l|}{$\mathrm{H}$} & \multicolumn{2}{|c|}{-1.43981200} & -0.26861100 & 1.59580200 \\
\hline 2050 & \multicolumn{2}{|l|}{$\mathrm{O}$} & \multicolumn{2}{|c|}{-1.61647400} & -1.67219500 & 0.06441400 \\
\hline 2051 & \multicolumn{2}{|l|}{$\mathrm{O}$} & \multicolumn{2}{|c|}{-2.75504400} & 0.24952000 & -0.20160000 \\
\hline 2052 & \multicolumn{2}{|l|}{$\mathrm{H}$} & \multicolumn{2}{|c|}{-3.47461700} & -0.39385600 & -0.16950200 \\
\hline 2053 & \multicolumn{2}{|c|}{ Frequencies $[1 / \mathrm{cm}]$} & & & & \\
\hline 2054 & -1025.75 & 340.41 & 804.59 & 1113.48 & $1341.38 \quad 2872.1$ & $11 \quad 2975.19$ \\
\hline 2055 & 56.54 & 384.22 & 836.68 & 1142.70 & $1345.67 \quad 2881.6$ & $67 \quad 3001.90$ \\
\hline 2056 & 80.52 & 471.54 & 854.91 & 1198.73 & $1408.31 \quad 2893.4$ & $44 \quad 3627.57$ \\
\hline 2057 & 96.18 & 522.76 & 907.85 & 1221.06 & $1420.17 \quad 2901.5$ & \\
\hline 2058 & 138.94 & 538.59 & 964.59 & 1253.71 & $\begin{array}{ll}1422.93 & 2902.3\end{array}$ & \\
\hline 2059 & 210.47 & 588.11 & 1001.69 & 1260.40 & $1426.07 \quad 2926.5$ & \\
\hline 2060 & 239.02 & 653.30 & 1026.68 & 1283.73 & $1432.48 \quad 2950.5$ & \\
\hline 2061 & 279.44 & 682.55 & 1072.86 & 1292.64 & $1435.56 \quad 2966.8$ & \\
\hline 2062 & \multirow[t]{2}{*}{313.45} & 710.62 & 1083.03 & 1336.32 & $1589.87 \quad 2973.2$ & \\
\hline \multicolumn{6}{|l|}{2063} & \\
\hline 2064 & \multicolumn{2}{|l|}{ acid } & & & & \\
\hline 2065 & \multicolumn{2}{|l|}{$\mathrm{C}$} & \multicolumn{2}{|c|}{0.86927800} & -0.38603400 & 0.05245100 \\
\hline 2066 & $\mathrm{H}$ & & & 4948600 & -1.32187600 & -0.51064500 \\
\hline 2067 & $\mathrm{H}$ & & & 4221700 & -0.67112500 & 1.10705600 \\
\hline 2068 & $\mathrm{C}$ & & & 6298800 & 0.35915700 & -0.25324600 \\
\hline 2069 & $\mathrm{H}$ & & & 4397600 & 1.24330800 & 0.38164200 \\
\hline 2070 & $\mathrm{H}$ & & & 3974900 & 0.71973400 & -1.28573200 \\
\hline 2071 & $\mathrm{C}$ & & & 8773600 & -0.52275200 & -0.04408300 \\
\hline 2072 & $\mathrm{H}$ & & & 5334800 & -1.39600300 & -0.69659800 \\
\hline 2073 & $\mathrm{H}$ & & & 1077400 & 0.01683900 & -0.25247800 \\
\hline 2074 & $\mathrm{H}$ & & & 3324800 & -0.88173300 & 0.98496600 \\
\hline 2075 & $\mathrm{C}$ & & -0.5 & 1581200 & 1.70012600 & 0.54759000 \\
\hline 2076 & $\mathrm{H}$ & & -0.4 & 7106800 & 1.47180000 & 1.61374300 \\
\hline 2077 & $\mathrm{H}$ & & & 9679800 & 2.38364500 & 0.31083800 \\
\hline 2078 & $\mathrm{H}$ & & -1.4 & 5683900 & 2.20477500 & 0.33847700 \\
\hline 2079 & $\mathrm{C}$ & & -0.3 & 3897500 & 0.41134400 & -0.27401000 \\
\hline 2080 & $\mathrm{H}$ & & -0.3 & 9302400 & 0.68134500 & -1.33369000 \\
\hline 2081 & $\mathrm{C}$ & & -1.6 & 1892900 & -0.43295900 & -0.04232200 \\
\hline 2082 & $\mathrm{O}$ & & -1.6 & 6200300 & -1.46697200 & 0.56455600 \\
\hline 2083 & $\mathrm{O}$ & & -2.7 & 2089100 & 0.12145900 & -0.58945100 \\
\hline 2084 & $\mathrm{H}$ & & -3.4 & 6323000 & -0.45989400 & -0.37670100 \\
\hline 2085 & Frequencies[ & $/ \mathrm{cm}]$ & & & & \\
\hline 2086 & 36.34 & 338.01 & 905.19 & 1219.42 & $1420.23 \quad 2904.8$ & \\
\hline 2087 & 63.29 & 508.54 & 957.91 & 1250.55 & $1425.47 \quad 2911.0$ & \\
\hline 2088 & 93.86 & 566.21 & 998.25 & 1260.87 & $1427.42 \quad 2923.1$ & \\
\hline 2089 & 131.46 & 606.32 & 1028.92 & 1282.19 & $1432.31 \quad 2944.6$ & \\
\hline 2090 & 199.78 & 704.00 & 1071.79 & 1318.97 & $1435.75 \quad 2966.4$ & \\
\hline 2091 & 234.55 & 731.66 & 1087.05 & 1337.96 & $\begin{array}{ll}1773.13 & 2973.4\end{array}$ & \\
\hline 2092 & 266.34 & 785.88 & 1113.55 & 1342.16 & $2888.80 \quad 2980.3$ & \\
\hline 2093 & 307.83 & 827.95 & 1145.18 & 1353.97 & $2900.00 \quad 2995.3$ & \\
\hline 2094 & 321.36 & 856.24 & 1201.84 & 1407.97 & $2901.98 \quad 3617.5$ & \\
\hline
\end{tabular}


TSdiss2

2097

2098

2099

2100

2101

2102

2103

2104

2105

2106

2107

2108

2109

2110

2111

2112

2113

2114

2115

2116

2117

2118

2119

2120

2121

2122

2123

2124

2125

2126

2127

2128

2129

2130

2131

2132

2133

2134

2135

2136

2137

2138

2139

2140

pentyl

C

$\mathrm{H}$

$\mathrm{H}$

C

$\mathrm{H}$

$\mathrm{H}$

C

$\mathrm{H}$

H

H

C

$$
\begin{array}{ccc}
0.93377400 & -0.33128400 & 0.15270700 \\
0.87077900 & -1.35555600 & -0.21333300 \\
0.92557000 & -0.38100500 & 1.24698100 \\
2.25309000 & 0.30162300 & -0.30346600 \\
2.34585300 & 1.30438500 & 0.11824500 \\
2.23855200 & 0.41941800 & -1.38998400 \\
3.45242800 & -0.54197300 & 0.10960600 \\
3.39388100 & -1.53871000 & -0.32851700 \\
4.38886100 & -0.08694000 & -0.21080100 \\
3.48821500 & -0.65822500 & 1.19354900 \\
-0.42230400 & 1.84339600 & 0.18736200 \\
-0.23488000 & 1.89591000 & 1.26148600 \\
0.30180800 & 2.49740300 & -0.30694000 \\
-1.41774800 & 2.22919400 & -0.02027300 \\
-0.26176400 & 0.44134000 & -0.32076700 \\
-0.47792700 & 0.32429300 & -1.38025200 \\
-1.80078700 & -0.51143400 & 0.33981100 \\
-1.56804300 & -0.22888400 & 1.37820700 \\
-1.62457300 & -1.66166600 & -0.08110400 \\
-2.86737000 & 0.23920100 & -0.10568800 \\
-3.24599800 & -0.25157300 & -0.84554500
\end{array}
$$

$\mathrm{H}$

uencies $[1 / \mathrm{cm}]$

$-374.37 \quad 314.14$

$\begin{array}{lllll}872.09 & 1137.18 & 1340.61 & 2880.61 & 2975.17\end{array}$

$\begin{array}{llllll}889.24 & 1205.67 & 1402.83 & 2886.32 & 2993.63\end{array}$

$\begin{array}{llllllll}67.68 & 369.09 & 920.74 & 1214.38 & 1410.29 & 2897.73 & 3633.31\end{array}$

$\begin{array}{lllllll}97.11 & 443.88 & 963.64 & 1239.30 & 1420.75 & 2902.91\end{array}$

$\begin{array}{lllllll}121.96 & 501.81 & 995.56 & 1258.93 & 1423.05 & 2929.01\end{array}$

$\begin{array}{lllllll}198.35 & 583.58 & 1012.00 & 1273.51 & 1425.27 & 2942.67\end{array}$

$\begin{array}{llllll}234.85 & 711.27 & 1047.20 & 1302.32 & 1434.62 & 2959.13\end{array}$

$\begin{array}{lllllll}240.97 & 819.39 & 1081.82 & 1316.29 & 1489.84 & 2969.33\end{array}$

$\begin{array}{llllll}262.47 & 838.53 & 1098.26 & 1335.42 & 2829.86 & 2974.52\end{array}$ 


$\begin{array}{llrrc}2141 & \mathrm{H} & 2.02700600 & -0.88322400 & 1.18045700 \\ 2142 & \mathrm{H} & 2.01583900 & -1.44416500 & -0.48364500 \\ 2143 & \mathrm{H} & 3.28378800 & -0.35457800 & 0.05316600 \\ 2144 & \mathrm{C} & 1.37942800 & 0.59926600 & -0.21601400 \\ 2145 & \mathrm{H} & 1.70239100 & 1.23661100 & -1.02790600\end{array}$

2146 Frequencies[1/cm]

2147

2148

$\begin{array}{lllll}62.45 & 817.74 & 1199.53 & 1414.44 & 2915.98\end{array}$

$\begin{array}{lllll}95.62 & 829.26 & 1231.80 & 1419.12 & 2935.44\end{array}$

2149

$\begin{array}{llllll}120.60 & 877.74 & 1258.46 & 1424.71 & 2960.27\end{array}$

2150

$\begin{array}{lllll}226.58 & 938.27 & 1297.26 & 1432.88 & 2963.63\end{array}$

2151

$\begin{array}{lllll}232.86 & 960.24 & 1315.64 & 2841.15 & 2970.21\end{array}$

2152

2153

$\begin{array}{lllll}309.66 & 1001.42 & 1338.29 & 2859.62 & 3028.54\end{array}$

2154

$\begin{array}{llll}395.50 & 1040.28 & 1343.65 & 2896.51\end{array}$

$\begin{array}{llll}463.30 & 1099.71 & 1398.32 & 2899.26\end{array}$

2155

$\begin{array}{llll}707.24 & 1120.45 & 1408.70 & 2910.38\end{array}$

2156

$2157 \mathrm{HCOOH}$

2158

2159

$\mathrm{C}$

0.00000000

0.38293000

0.00000000

$\mathrm{H}$

$-0.46257500$

1.38230700

0.00000000

2160

$\mathrm{O}$

1.17171800

0.19751900

0.00000000

2161

$\mathrm{O}$

$-0.89052900$

$-0.62328600$

0.00000000

2162

$\mathrm{H}$

$-1.78693800$

$-0.27375300$

0.00000000

Frequencies[1/cm]

2164

$\begin{array}{lllll}491.38 & 640.71 & 1005.61 & 1087.47 & 1227.33\end{array}$

2165

$\begin{array}{llll}1363.77 & 1823.36 & 2864.66 & 3672.99\end{array}$

2166

2167

2). Table S2. The structures of various transition states, reaction complexes(RCs) and product 2168 complexes (PCs) on 3-MH $+\mathrm{OH}$ and 2-MP $+\mathrm{OH}$ potential energy surface.

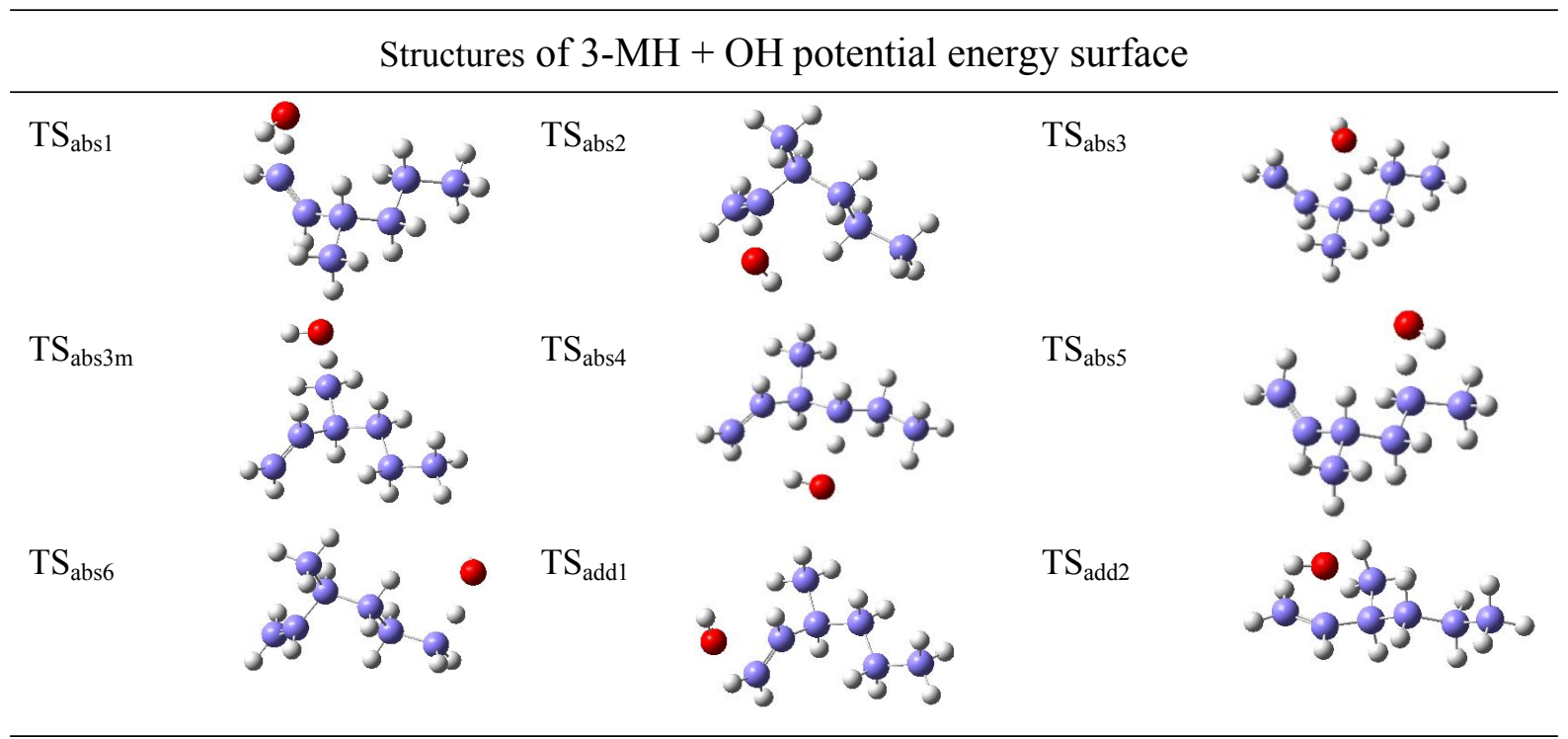




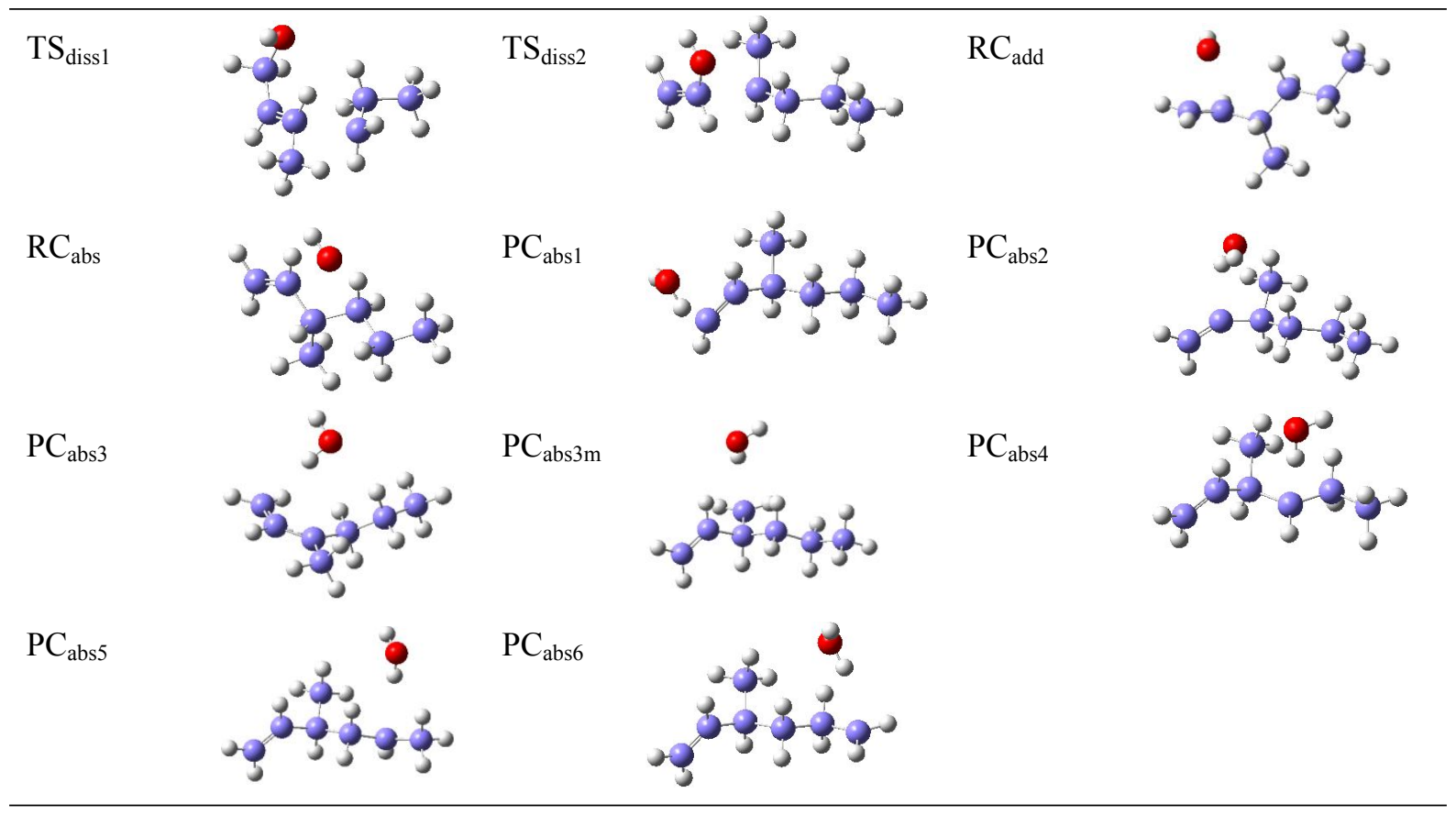

Structures of 2-MP $+\mathrm{OH}$ potential energy surface

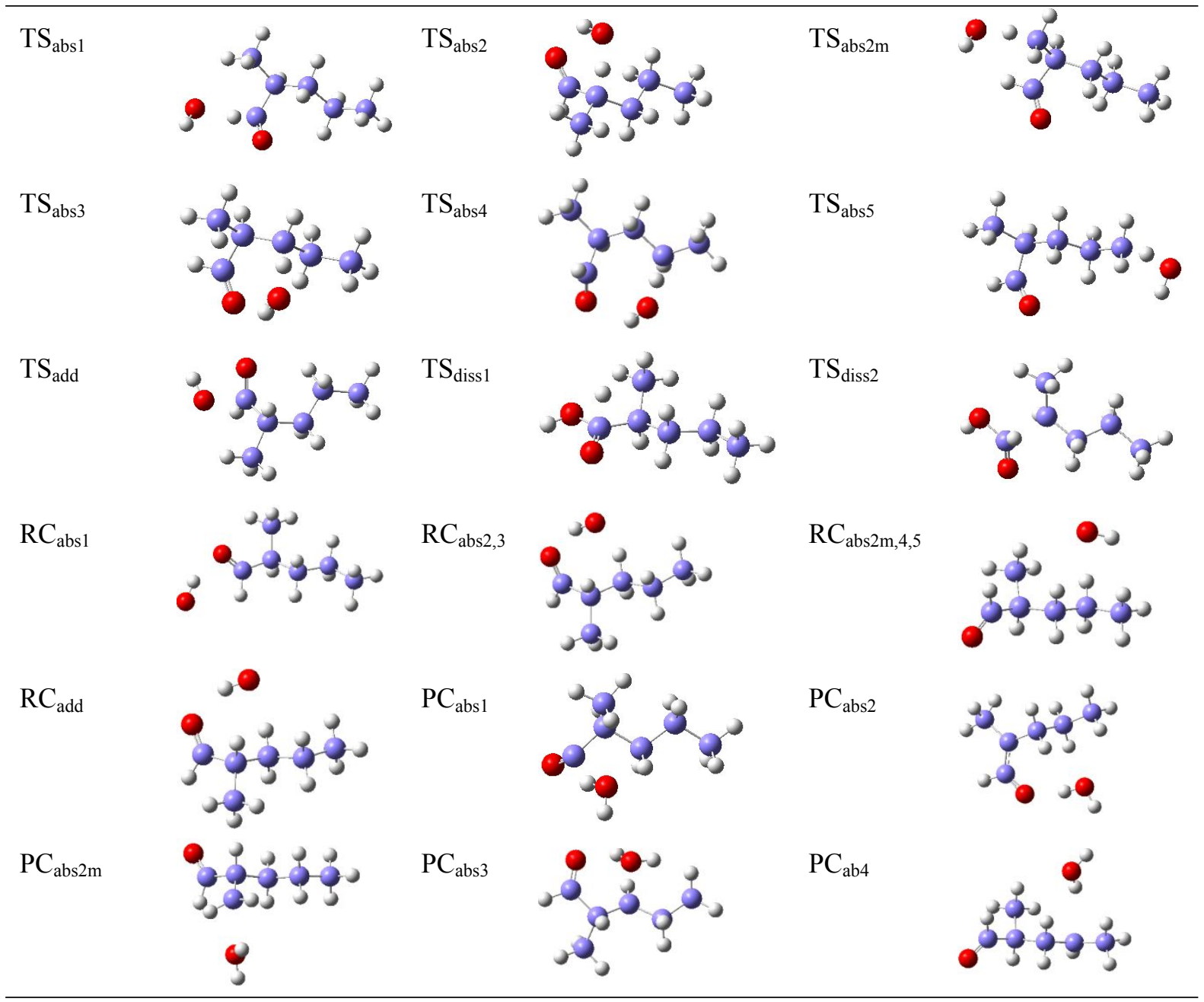


$\mathrm{PC}_{\mathrm{abs} 5}$

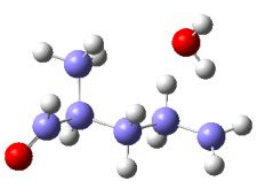

2173 3). Table S3. T1 diagnostic, spin expectation values and point group of the stationary points

\begin{tabular}{|c|c|c|c|c|c|c|c|}
\hline species & T1 value & $<\mathrm{S}^{2}>$ & point group & species & T1 value & $<\mathrm{S}^{2}>$ & point group \\
\hline 3-MH & 0.009 & I & $\mathrm{C} 1$ & PCabs5 & 0.011 & 0.755 & $\mathrm{C} 1$ \\
\hline RCabs & 0.010 & 0.753 & $\mathrm{C} 1$ & PCabs6 & 0.011 & 0.755 & $\mathrm{C} 1$ \\
\hline RCadd & 0.013 & 0.755 & $\mathrm{C} 1$ & $\mathrm{OH}$ & 0.009 & 0.752 & $\mathrm{C} \infty \mathrm{v}$ \\
\hline TSabs 1 & 0.021 & 0.760 & $\mathrm{C} 1$ & 2-MP & 0.012 & / & $\mathrm{C} 1$ \\
\hline TSabs2 & 0.021 & 0.759 & $\mathrm{C} 1$ & RCabs1 & 0.012 & 0.753 & $\mathrm{C} 1$ \\
\hline TSabs3 & 0.019 & 0.758 & $\mathrm{C} 1$ & RCabs 23 & 0.012 & 0.753 & $\mathrm{C} 1$ \\
\hline TSabs3m & 0.019 & 0.758 & $\mathrm{C} 1$ & RCabs $2 \mathrm{~m} 45$ & 0.012 & 0.753 & $\mathrm{C} 1$ \\
\hline TSabs4 & 0.020 & 0.758 & $\mathrm{C} 1$ & RCaddC & 0.012 & 0.755 & $\mathrm{C} 1$ \\
\hline TSabs5 & 0.019 & 0.757 & $\mathrm{C} 1$ & TSabs 1 & 0.019 & 0.758 & $\mathrm{C} 1$ \\
\hline TSabs6 & 0.019 & 0.758 & $\mathrm{C} 1$ & TSabs 2 & 0.018 & 0.758 & $\mathrm{C} 1$ \\
\hline TSadd 1 & 0.021 & 0.768 & $\mathrm{C} 1$ & TSabs $2 \mathrm{~m}$ & 0.017 & 0.758 & $\mathrm{C} 1$ \\
\hline TSadd2 & 0.043 & 0.766 & $\mathrm{C} 1$ & TSabs 3 & 0.017 & 0.758 & $\mathrm{C} 1$ \\
\hline TSdiss 1 & 0.018 & 0.780 & $\mathrm{C} 1$ & TSabs4 & 0.017 & 0.758 & $\mathrm{C} 1$ \\
\hline TSdiss2 & 0.019 & 0.779 & $\mathrm{C} 1$ & TSabs5 & 0.017 & 0.758 & $\mathrm{C} 1$ \\
\hline abs1 & 0.012 & 0.765 & $\mathrm{C} 1$ & TSaddC & 0.031 & 0.776 & $\mathrm{C} 1$ \\
\hline abs2 & 0.012 & 0.765 & $\mathrm{C} 1$ & abs 1 & 0.014 & 0.753 & $\mathrm{C} 1$ \\
\hline abs3 & 0.016 & 0.778 & $\mathrm{C} 1$ & abs2 & 0.021 & 0.767 & $\mathrm{C} 1$ \\
\hline abs3m & 0.011 & 0.755 & $\mathrm{C} 1$ & abs $2 \mathrm{~m}$ & 0.014 & 0.757 & $\mathrm{C} 1$ \\
\hline abs4 & 0.011 & 0.755 & $\mathrm{C} 1$ & abs3 & 0.015 & 0.755 & $\mathrm{C} 1$ \\
\hline abs5 & 0.011 & 0.755 & $\mathrm{C} 1$ & abs 4 & 0.013 & 0.755 & $\mathrm{C} 1$ \\
\hline abs6 & 0.011 & 0.755 & $\mathrm{C} 1$ & abs5 & 0.013 & 0.755 & $\mathrm{C} 1$ \\
\hline add1 & 0.011 & 0.755 & $\mathrm{C} 1$ & addC & 0.017 & 0.756 & $\mathrm{C} 1$ \\
\hline add2 & 0.011 & 0.755 & $\mathrm{C} 1$ & TSdiss-1 & 0.018 & 0.764 & $\mathrm{C} 1$ \\
\hline diss 1-1 & 0.011 & / & $\mathrm{C} 1$ & pentyl & 0.011 & 0.755 & $\mathrm{C} 1$ \\
\hline diss $1-2$ & 0.011 & 0.755 & $\mathrm{C} 1$ & TSdiss-2 & 0.018 & 0.765 & $\mathrm{C} 1$ \\
\hline diss2-1 & 0.013 & I & $\mathrm{C} 1$ & $\mathrm{HCOOH}$ & 0.016 & / & Cs \\
\hline diss $2-2$ & 0.011 & 0.755 & $\mathrm{C} 1$ & acid & 0.012 & / & $\mathrm{C} 1$ \\
\hline $\mathrm{H} 2 \mathrm{O}$ & 0.010 & / & $\mathrm{C} 2 \mathrm{v}$ & PCabs1 & 0.014 & 0.753 & $\mathrm{C} 1$ \\
\hline PCabs1 & 0.021 & 0.765 & $\mathrm{C} 1$ & PCabs2 & 0.018 & 0.764 & $\mathrm{C} 1$ \\
\hline PCabs2 & 0.012 & 0.764 & $\mathrm{C} 1$ & PCabs2m & 0.013 & 0.755 & $\mathrm{C} 1$ \\
\hline PCabs3 & 0.014 & 0.778 & $\mathrm{C} 1$ & PCabs3 & 0.013 & 0.755 & $\mathrm{C} 1$ \\
\hline
\end{tabular}




\begin{tabular}{|c|c|c|c|c|c|c|c|}
\hline PCabs3m & 0.011 & 0.755 & $\mathrm{C} 1$ & PCabs4 & 0.013 & 0.755 & $\mathrm{C} 1$ \\
\hline PCabs4 & 0.011 & 0.755 & $\mathrm{C} 1$ & PCabs5 & 0.013 & 0.755 & $\mathrm{C} 1$ \\
\hline
\end{tabular}

2176 4). Table S4.1 Final rate coefficients of 3-MH+OH $\left(\mathrm{cm}^{3}\right.$ molecule $\left.{ }^{-1} \mathrm{~s}^{-1}\right)$ of various channels at $2177 \quad 1.0 \mathrm{~atm}$

\begin{tabular}{|c|c|c|c|c|c|}
\hline $\mathrm{T}(\mathrm{K})$ & R->P-add1 & R->P-add2 & $\mathrm{R}->\mathrm{P}-\mathrm{abs} 1$ & $\mathrm{R}->\mathrm{P}-\mathrm{abs} 2$ & $\mathrm{R}->\mathrm{P}-\mathrm{abs} 3$ \\
\hline 200 & $3.3 \mathrm{E}-11$ & $3.0 \mathrm{E}-12$ & $1.7 \mathrm{E}-15$ & $1.5 \mathrm{E}-14$ & $3.0 \mathrm{E}-12$ \\
\hline 210 & 3.4E-11 & $3.1 \mathrm{E}-12$ & $2.2 \mathrm{E}-15$ & $1.9 \mathrm{E}-14$ & $3.0 \mathrm{E}-12$ \\
\hline 220 & $3.5 \mathrm{E}-11$ & $3.2 \mathrm{E}-12$ & $2.8 \mathrm{E}-15$ & $2.2 \mathrm{E}-14$ & $2.9 \mathrm{E}-12$ \\
\hline 230 & $3.5 \mathrm{E}-11$ & $3.3 \mathrm{E}-12$ & $3.7 \mathrm{E}-15$ & 2.7E-14 & $2.9 \mathrm{E}-12$ \\
\hline 240 & $3.6 \mathrm{E}-11$ & $3.4 \mathrm{E}-12$ & 4.7E-15 & $3.2 \mathrm{E}-14$ & $2.8 \mathrm{E}-12$ \\
\hline 250 & $3.6 \mathrm{E}-11$ & $3.5 \mathrm{E}-12$ & $5.9 \mathrm{E}-15$ & $3.7 \mathrm{E}-14$ & $2.8 \mathrm{E}-12$ \\
\hline 260 & 3.7E-11 & $3.5 \mathrm{E}-12$ & 7.3E-15 & $4.3 \mathrm{E}-14$ & $2.7 \mathrm{E}-12$ \\
\hline 270 & $3.7 \mathrm{E}-11$ & $3.6 \mathrm{E}-12$ & $9.1 \mathrm{E}-15$ & $5.0 \mathrm{E}-14$ & 2.6E-12 \\
\hline 280 & $3.7 \mathrm{E}-11$ & $3.6 \mathrm{E}-12$ & $1.1 \mathrm{E}-14$ & $5.8 \mathrm{E}-14$ & $2.6 \mathrm{E}-12$ \\
\hline 290 & $3.7 \mathrm{E}-11$ & $3.7 \mathrm{E}-12$ & $1.4 \mathrm{E}-14$ & $6.6 \mathrm{E}-14$ & $2.5 \mathrm{E}-12$ \\
\hline 300 & $3.7 \mathrm{E}-11$ & $3.7 \mathrm{E}-12$ & $1.6 \mathrm{E}-14$ & $7.5 \mathrm{E}-14$ & $2.5 \mathrm{E}-12$ \\
\hline 310 & $3.8 \mathrm{E}-11$ & $3.8 \mathrm{E}-12$ & $2.0 \mathrm{E}-14$ & $8.5 \mathrm{E}-14$ & $2.4 \mathrm{E}-12$ \\
\hline 320 & $3.8 \mathrm{E}-11$ & $3.8 \mathrm{E}-12$ & $2.3 \mathrm{E}-14$ & $9.6 \mathrm{E}-14$ & $2.4 \mathrm{E}-12$ \\
\hline 330 & $3.8 \mathrm{E}-11$ & $3.9 \mathrm{E}-12$ & $2.8 \mathrm{E}-14$ & $1.1 \mathrm{E}-13$ & 2.3E-12 \\
\hline 340 & 3.7E-11 & $3.9 \mathrm{E}-12$ & $3.2 \mathrm{E}-14$ & $1.2 \mathrm{E}-13$ & $2.3 \mathrm{E}-12$ \\
\hline 350 & 3.7E-11 & $3.9 \mathrm{E}-12$ & $3.8 \mathrm{E}-14$ & $1.3 \mathrm{E}-13$ & 2.2E-12 \\
\hline 360 & $3.7 \mathrm{E}-11$ & $3.9 \mathrm{E}-12$ & $4.4 \mathrm{E}-14$ & $1.5 \mathrm{E}-13$ & 2.2E-12 \\
\hline 370 & $3.7 \mathrm{E}-11$ & $3.9 \mathrm{E}-12$ & $5.0 \mathrm{E}-14$ & $1.6 \mathrm{E}-13$ & 2.1E-12 \\
\hline 380 & 3.7E-11 & $4.0 \mathrm{E}-12$ & $5.8 \mathrm{E}-14$ & $1.8 \mathrm{E}-13$ & $2.1 \mathrm{E}-12$ \\
\hline 390 & $3.7 \mathrm{E}-11$ & $4.0 \mathrm{E}-12$ & $6.6 \mathrm{E}-14$ & $2.0 \mathrm{E}-13$ & $2.0 \mathrm{E}-12$ \\
\hline 400 & $3.7 \mathrm{E}-11$ & $4.0 \mathrm{E}-12$ & $7.5 \mathrm{E}-14$ & $2.2 \mathrm{E}-13$ & $2.0 \mathrm{E}-12$ \\
\hline 410 & $3.6 \mathrm{E}-11$ & $4.0 \mathrm{E}-12$ & 8.4E-14 & $2.4 \mathrm{E}-13$ & $1.9 \mathrm{E}-12$ \\
\hline 420 & $3.6 \mathrm{E}-11$ & $4.0 \mathrm{E}-12$ & $9.5 \mathrm{E}-14$ & $2.6 \mathrm{E}-13$ & $1.9 \mathrm{E}-12$ \\
\hline 430 & 3.6E-11 & $4.0 \mathrm{E}-12$ & $1.1 \mathrm{E}-13$ & $2.8 \mathrm{E}-13$ & $1.9 \mathrm{E}-12$ \\
\hline 440 & $3.6 \mathrm{E}-11$ & $4.0 \mathrm{E}-12$ & $1.2 \mathrm{E}-13$ & $3.0 \mathrm{E}-13$ & $1.8 \mathrm{E}-12$ \\
\hline 450 & $3.5 \mathrm{E}-11$ & $4.0 \mathrm{E}-12$ & $1.3 \mathrm{E}-13$ & $3.3 \mathrm{E}-13$ & $1.8 \mathrm{E}-12$ \\
\hline 500 & 3.4E-11 & $3.9 \mathrm{E}-12$ & $2.2 \mathrm{E}-13$ & $4.7 \mathrm{E}-13$ & $1.7 \mathrm{E}-12$ \\
\hline 550 & $3.2 \mathrm{E}-11$ & $3.9 \mathrm{E}-12$ & $3.3 \mathrm{E}-13$ & $6.5 \mathrm{E}-13$ & $1.6 \mathrm{E}-12$ \\
\hline 600 & $3.1 \mathrm{E}-11$ & $3.8 \mathrm{E}-12$ & $4.8 \mathrm{E}-13$ & $8.6 \mathrm{E}-13$ & $1.5 \mathrm{E}-12$ \\
\hline 650 & $3.0 \mathrm{E}-11$ & $3.7 \mathrm{E}-12$ & $6.6 \mathrm{E}-13$ & $1.1 \mathrm{E}-12$ & $1.5 \mathrm{E}-12$ \\
\hline 700 & $2.8 \mathrm{E}-11$ & $3.7 \mathrm{E}-12$ & $8.8 \mathrm{E}-13$ & $1.4 \mathrm{E}-12$ & $1.5 \mathrm{E}-12$ \\
\hline 750 & 2.7E-11 & $3.7 \mathrm{E}-12$ & $1.2 \mathrm{E}-12$ & $1.7 \mathrm{E}-12$ & $1.5 \mathrm{E}-12$ \\
\hline 800 & $2.5 \mathrm{E}-11$ & $3.7 \mathrm{E}-12$ & $1.5 \mathrm{E}-12$ & $2.1 \mathrm{E}-12$ & $1.4 \mathrm{E}-12$ \\
\hline 850 & $2.4 \mathrm{E}-11$ & $3.8 \mathrm{E}-12$ & $1.8 \mathrm{E}-12$ & $2.5 \mathrm{E}-12$ & $1.4 \mathrm{E}-12$ \\
\hline 900 & $2.2 \mathrm{E}-11$ & $3.8 \mathrm{E}-12$ & $2.2 \mathrm{E}-12$ & $2.9 \mathrm{E}-12$ & $1.5 \mathrm{E}-12$ \\
\hline 950 & $1.9 \mathrm{E}-11$ & $3.8 \mathrm{E}-12$ & $2.7 \mathrm{E}-12$ & $3.4 \mathrm{E}-12$ & $1.5 \mathrm{E}-12$ \\
\hline
\end{tabular}




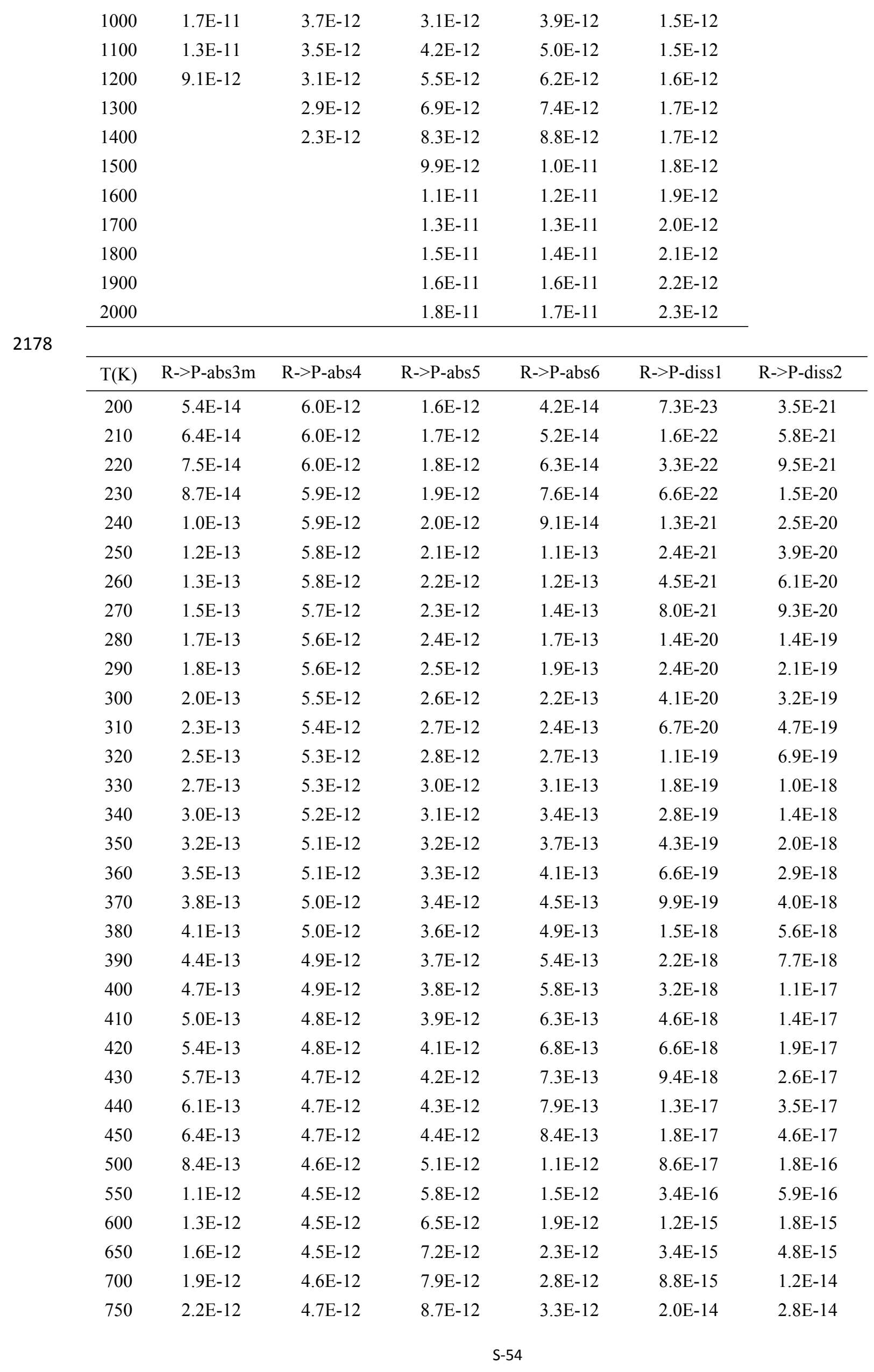




\begin{tabular}{ccccccc}
800 & $2.5 \mathrm{E}-12$ & $4.8 \mathrm{E}-12$ & $9.5 \mathrm{E}-12$ & $3.8 \mathrm{E}-12$ & $4.1 \mathrm{E}-14$ & $5.8 \mathrm{E}-14$ \\
850 & $2.8 \mathrm{E}-12$ & $4.9 \mathrm{E}-12$ & $1.0 \mathrm{E}-11$ & $4.3 \mathrm{E}-12$ & $7.4 \mathrm{E}-14$ & $1.1 \mathrm{E}-13$ \\
900 & $3.2 \mathrm{E}-12$ & $5.0 \mathrm{E}-12$ & $1.1 \mathrm{E}-11$ & $4.9 \mathrm{E}-12$ & $1.2 \mathrm{E}-13$ & $2.0 \mathrm{E}-13$ \\
950 & $3.6 \mathrm{E}-12$ & $5.1 \mathrm{E}-12$ & $1.2 \mathrm{E}-11$ & $5.5 \mathrm{E}-12$ & $1.9 \mathrm{E}-13$ & $3.2 \mathrm{E}-13$ \\
1000 & $4.0 \mathrm{E}-12$ & $5.3 \mathrm{E}-12$ & $1.3 \mathrm{E}-11$ & $6.1 \mathrm{E}-12$ & $2.7 \mathrm{E}-13$ & $4.8 \mathrm{E}-13$ \\
1100 & $4.8 \mathrm{E}-12$ & $5.6 \mathrm{E}-12$ & $1.4 \mathrm{E}-11$ & $7.4 \mathrm{E}-12$ & $4.7 \mathrm{E}-13$ & $9.1 \mathrm{E}-13$ \\
1200 & $5.6 \mathrm{E}-12$ & $6.0 \mathrm{E}-12$ & $1.6 \mathrm{E}-11$ & $8.7 \mathrm{E}-12$ & $7.2 \mathrm{E}-13$ & $1.4 \mathrm{E}-12$ \\
1300 & $6.5 \mathrm{E}-12$ & $6.4 \mathrm{E}-12$ & $1.7 \mathrm{E}-11$ & $1.0 \mathrm{E}-11$ & $1.1 \mathrm{E}-12$ & $1.9 \mathrm{E}-12$ \\
1400 & $7.4 \mathrm{E}-12$ & $6.9 \mathrm{E}-12$ & $1.9 \mathrm{E}-11$ & $1.1 \mathrm{E}-11$ & $1.4 \mathrm{E}-12$ & $3.3 \mathrm{E}-12$ \\
1500 & $8.3 \mathrm{E}-12$ & $7.4 \mathrm{E}-12$ & $2.0 \mathrm{E}-11$ & $1.3 \mathrm{E}-11$ & $1.7 \mathrm{E}-12$ & $3.5 \mathrm{E}-12$ \\
1600 & $9.2 \mathrm{E}-12$ & $7.9 \mathrm{E}-12$ & $2.2 \mathrm{E}-11$ & $1.4 \mathrm{E}-11$ & $2.0 \mathrm{E}-12$ & $3.7 \mathrm{E}-12$ \\
1700 & $1.0 \mathrm{E}-11$ & $8.4 \mathrm{E}-12$ & $2.3 \mathrm{E}-11$ & $1.5 \mathrm{E}-11$ & $2.3 \mathrm{E}-12$ & $4.0 \mathrm{E}-12$ \\
1800 & $1.1 \mathrm{E}-11$ & $9.0 \mathrm{E}-12$ & $2.4 \mathrm{E}-11$ & $1.6 \mathrm{E}-11$ & $2.7 \mathrm{E}-12$ & $4.2 \mathrm{E}-12$ \\
1900 & $1.2 \mathrm{E}-11$ & $9.6 \mathrm{E}-12$ & $2.5 \mathrm{E}-11$ & $1.7 \mathrm{E}-11$ & $3.0 \mathrm{E}-12$ & $4.5 \mathrm{E}-12$ \\
2000 & $1.3 \mathrm{E}-11$ & $1.0 \mathrm{E}-11$ & $2.6 \mathrm{E}-11$ & $1.8 \mathrm{E}-11$ & $3.4 \mathrm{E}-12$ & $4.7 \mathrm{E}-12$ \\
\hline
\end{tabular}

2179

2180

2181 Table S4.2 Final rate coefficients of 2-MP+OH $\left(\mathrm{cm}^{3}\right.$ molecule $\left.\mathrm{e}^{-1} \mathrm{~s}^{-1}\right)$ of various channels at 1.0

$2182 \mathrm{~atm}$

\begin{tabular}{rrrrrrr}
\hline $\mathrm{T}(\mathrm{K})$ & R->P-add & R->P-abs1 & R->P-abs2 & R->P-abs2m & R->P-abs3 & R->P-abs4 \\
\hline 200 & $4.0 \mathrm{E}-18$ & $7.3 \mathrm{E}-12$ & $1.3 \mathrm{E}-11$ & $2.8 \mathrm{E}-15$ & $8.1 \mathrm{E}-12$ & $2.8 \mathrm{E}-12$ \\
210 & $5.9 \mathrm{E}-18$ & $6.9 \mathrm{E}-12$ & $1.3 \mathrm{E}-11$ & $3.2 \mathrm{E}-15$ & $7.8 \mathrm{E}-12$ & $2.6 \mathrm{E}-12$ \\
220 & $8.6 \mathrm{E}-18$ & $6.3 \mathrm{E}-12$ & $1.3 \mathrm{E}-11$ & $3.7 \mathrm{E}-15$ & $7.6 \mathrm{E}-12$ & $2.4 \mathrm{E}-12$ \\
230 & $1.2 \mathrm{E}-17$ & $5.8 \mathrm{E}-12$ & $1.4 \mathrm{E}-11$ & $4.3 \mathrm{E}-15$ & $7.3 \mathrm{E}-12$ & $2.2 \mathrm{E}-12$ \\
240 & $1.7 \mathrm{E}-17$ & $5.3 \mathrm{E}-12$ & $1.4 \mathrm{E}-11$ & $4.9 \mathrm{E}-15$ & $7.0 \mathrm{E}-12$ & $2.1 \mathrm{E}-12$ \\
250 & $2.3 \mathrm{E}-17$ & $5.3 \mathrm{E}-12$ & $1.4 \mathrm{E}-11$ & $5.6 \mathrm{E}-15$ & $6.7 \mathrm{E}-12$ & $2.0 \mathrm{E}-12$ \\
260 & $3.1 \mathrm{E}-17$ & $4.8 \mathrm{E}-12$ & $1.4 \mathrm{E}-11$ & $6.3 \mathrm{E}-15$ & $6.4 \mathrm{E}-12$ & $1.9 \mathrm{E}-12$ \\
270 & $4.0 \mathrm{E}-17$ & $4.4 \mathrm{E}-12$ & $1.4 \mathrm{E}-11$ & $7.2 \mathrm{E}-15$ & $6.1 \mathrm{E}-12$ & $1.8 \mathrm{E}-12$ \\
280 & $5.1 \mathrm{E}-17$ & $4.1 \mathrm{E}-12$ & $1.4 \mathrm{E}-11$ & $8.1 \mathrm{E}-15$ & $5.9 \mathrm{E}-12$ & $1.8 \mathrm{E}-12$ \\
290 & $6.5 \mathrm{E}-17$ & $3.7 \mathrm{E}-12$ & $1.4 \mathrm{E}-11$ & $9.1 \mathrm{E}-15$ & $5.6 \mathrm{E}-12$ & $1.7 \mathrm{E}-12$ \\
300 & $8.2 \mathrm{E}-17$ & $3.5 \mathrm{E}-12$ & $1.4 \mathrm{E}-11$ & $1.0 \mathrm{E}-14$ & $5.3 \mathrm{E}-12$ & $1.7 \mathrm{E}-12$ \\
310 & $1.0 \mathrm{E}-16$ & $3.2 \mathrm{E}-12$ & $1.4 \mathrm{E}-11$ & $1.1 \mathrm{E}-14$ & $5.1 \mathrm{E}-12$ & $1.6 \mathrm{E}-12$ \\
320 & $1.2 \mathrm{E}-16$ & $3.0 \mathrm{E}-12$ & $1.4 \mathrm{E}-11$ & $1.3 \mathrm{E}-14$ & $4.8 \mathrm{E}-12$ & $1.6 \mathrm{E}-12$ \\
330 & $1.5 \mathrm{E}-16$ & $2.8 \mathrm{E}-12$ & $1.3 \mathrm{E}-11$ & $1.4 \mathrm{E}-14$ & $4.6 \mathrm{E}-12$ & $1.6 \mathrm{E}-12$ \\
340 & $1.8 \mathrm{E}-16$ & $2.6 \mathrm{E}-12$ & $1.3 \mathrm{E}-11$ & $1.5 \mathrm{E}-14$ & $4.4 \mathrm{E}-12$ & $1.5 \mathrm{E}-12$ \\
350 & $2.1 \mathrm{E}-16$ & $2.5 \mathrm{E}-12$ & $1.3 \mathrm{E}-11$ & $1.7 \mathrm{E}-14$ & $4.2 \mathrm{E}-12$ & $1.5 \mathrm{E}-12$ \\
360 & $2.5 \mathrm{E}-16$ & $2.3 \mathrm{E}-12$ & $1.3 \mathrm{E}-11$ & $1.9 \mathrm{E}-14$ & $4.0 \mathrm{E}-12$ & $1.5 \mathrm{E}-12$ \\
370 & $3.0 \mathrm{E}-16$ & $2.2 \mathrm{E}-12$ & $1.3 \mathrm{E}-11$ & $2.1 \mathrm{E}-14$ & $3.8 \mathrm{E}-12$ & $1.5 \mathrm{E}-12$ \\
380 & $3.5 \mathrm{E}-16$ & $2.1 \mathrm{E}-12$ & $1.2 \mathrm{E}-11$ & $2.2 \mathrm{E}-14$ & $3.6 \mathrm{E}-12$ & $1.5 \mathrm{E}-12$ \\
390 & $4.0 \mathrm{E}-16$ & $2.0 \mathrm{E}-12$ & $1.2 \mathrm{E}-11$ & $2.4 \mathrm{E}-14$ & $3.5 \mathrm{E}-12$ & $1.5 \mathrm{E}-12$ \\
400 & $4.7 \mathrm{E}-16$ & $1.9 \mathrm{E}-12$ & $1.2 \mathrm{E}-11$ & $2.7 \mathrm{E}-14$ & $3.3 \mathrm{E}-12$ & $1.5 \mathrm{E}-12$ \\
410 & $5.3 \mathrm{E}-16$ & $1.8 \mathrm{E}-12$ & $1.2 \mathrm{E}-11$ & $2.9 \mathrm{E}-14$ & $3.2 \mathrm{E}-12$ & $1.5 \mathrm{E}-12$ \\
420 & $6.1 \mathrm{E}-16$ & $1.7 \mathrm{E}-12$ & $1.1 \mathrm{E}-11$ & $3.1 \mathrm{E}-14$ & $3.1 \mathrm{E}-12$ & $1.5 \mathrm{E}-12$
\end{tabular}




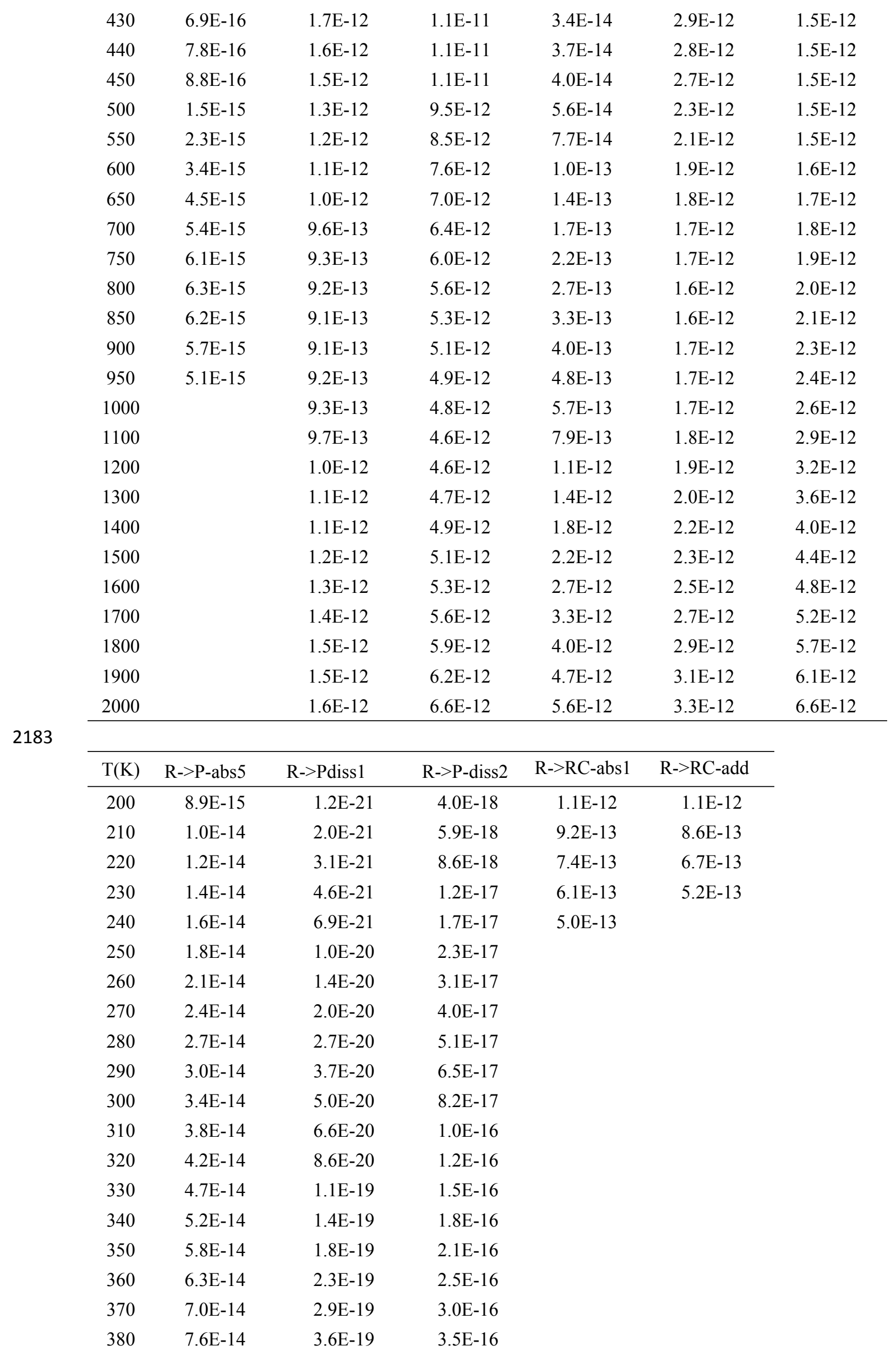




\begin{tabular}{|c|c|c|c|}
\hline 390 & $8.3 \mathrm{E}-14$ & 4.4E-19 & $4.0 \mathrm{E}-16$ \\
\hline 400 & $9.1 \mathrm{E}-14$ & $5.4 \mathrm{E}-19$ & $4.7 \mathrm{E}-16$ \\
\hline 410 & $9.8 \mathrm{E}-14$ & $6.6 \mathrm{E}-19$ & $5.3 \mathrm{E}-16$ \\
\hline 420 & $1.1 \mathrm{E}-13$ & 8.1E-19 & $6.1 \mathrm{E}-16$ \\
\hline 430 & $1.2 \mathrm{E}-13$ & $9.7 \mathrm{E}-19$ & $6.9 \mathrm{E}-16$ \\
\hline 440 & $1.2 \mathrm{E}-13$ & $1.2 \mathrm{E}-18$ & $7.8 \mathrm{E}-16$ \\
\hline 450 & $1.3 \mathrm{E}-13$ & $1.4 \mathrm{E}-18$ & $8.8 \mathrm{E}-16$ \\
\hline 500 & $1.9 \mathrm{E}-13$ & $3.2 \mathrm{E}-18$ & $1.5 \mathrm{E}-15$ \\
\hline 550 & $2.6 \mathrm{E}-13$ & $6.8 \mathrm{E}-18$ & $2.4 \mathrm{E}-15$ \\
\hline 600 & $3.5 \mathrm{E}-13$ & $1.3 \mathrm{E}-17$ & $3.6 \mathrm{E}-15$ \\
\hline 650 & $4.5 \mathrm{E}-13$ & $2.4 \mathrm{E}-17$ & $5.2 \mathrm{E}-15$ \\
\hline 700 & $5.7 \mathrm{E}-13$ & 4.3E-17 & 7.3E-15 \\
\hline 750 & $7.0 \mathrm{E}-13$ & $7.1 \mathrm{E}-17$ & $9.8 \mathrm{E}-15$ \\
\hline 800 & $8.6 \mathrm{E}-13$ & $1.1 \mathrm{E}-16$ & $1.3 \mathrm{E}-14$ \\
\hline 850 & $1.0 \mathrm{E}-12$ & $1.7 \mathrm{E}-16$ & $1.7 \mathrm{E}-14$ \\
\hline 900 & $1.2 \mathrm{E}-12$ & $2.6 \mathrm{E}-16$ & $2.1 \mathrm{E}-14$ \\
\hline 950 & $1.5 \mathrm{E}-12$ & $3.8 \mathrm{E}-16$ & $2.6 \mathrm{E}-14$ \\
\hline 1000 & $1.7 \mathrm{E}-12$ & $5.4 \mathrm{E}-16$ & $3.1 \mathrm{E}-14$ \\
\hline 1100 & $2.3 \mathrm{E}-12$ & $1.0 \mathrm{E}-15$ & $4.5 \mathrm{E}-14$ \\
\hline 1200 & $3.0 \mathrm{E}-12$ & $1.8 \mathrm{E}-15$ & $6.2 \mathrm{E}-14$ \\
\hline 1300 & $3.8 \mathrm{E}-12$ & $2.9 \mathrm{E}-15$ & 8.2E-14 \\
\hline 1400 & $4.8 \mathrm{E}-12$ & $4.5 \mathrm{E}-15$ & $1.1 \mathrm{E}-13$ \\
\hline 1500 & $5.9 \mathrm{E}-12$ & $6.8 \mathrm{E}-15$ & $1.3 \mathrm{E}-13$ \\
\hline 1600 & $7.1 \mathrm{E}-12$ & $9.7 \mathrm{E}-15$ & $1.7 \mathrm{E}-13$ \\
\hline 1700 & $8.5 \mathrm{E}-12$ & $1.4 \mathrm{E}-14$ & $2.0 \mathrm{E}-13$ \\
\hline 1800 & $1.0 \mathrm{E}-11$ & $1.8 \mathrm{E}-14$ & $2.4 \mathrm{E}-13$ \\
\hline 1900 & $1.2 \mathrm{E}-11$ & $2.4 \mathrm{E}-14$ & $2.8 \mathrm{E}-13$ \\
\hline 2000 & $1.3 \mathrm{E}-11$ & $3.1 \mathrm{E}-14$ & $3.3 \mathrm{E}-13$ \\
\hline
\end{tabular}

2184

2185 5). Table S5.1 The computed RRKM/ME rate coefficients $\left(\mathrm{cm}^{3}\right.$ molecule $\left.{ }^{-1} \mathrm{~s}^{-1}\right)$ for $3-\mathrm{MH}+\mathrm{OH}$ 2186 in the format of $\mathrm{k}=\mathrm{A} \cdot \mathrm{T}^{\mathrm{n}} \cdot \exp \left(-\mathrm{E}_{\mathrm{a}} / \mathrm{RT}\right)$ at $200-1000 \mathrm{~K}, 1 \mathrm{~atm}$.

\begin{tabular}{cccc}
\hline 200-450K & $\mathrm{A}\left(\mathrm{cm}^{3}\right.$ molecule $\left.{ }^{-1}\right)$ & $\mathrm{n}$ & $\mathrm{E}_{\mathrm{a}}\left(\mathrm{kcal} \mathrm{mol}^{-1}\right)$ \\
\hline R->P-add1 & $3.54 \mathrm{E}-11$ & 0.04 & 0.12 \\
R->P-add2 & $7.89 \mathrm{E}-13$ & 0.28 & 0.02 \\
R->P-abs1 & $1.98 \mathrm{E}-21$ & 3.22 & 1.45 \\
R->P-abs2 & $8.95 \mathrm{E}-20$ & 2.61 & 0.74 \\
R->P-abs3 & $2.84 \mathrm{E}-12$ & -0.11 & -0.28 \\
R->P-abs3m & $3.52 \mathrm{E}-17$ & 1.75 & 0.78 \\
R->P-abs4 & $3.79 \mathrm{E}-12$ & 0.09 & 0.12 \\
R->P-abs5 & $1.42 \mathrm{E}-14$ & 0.96 & 0.15 \\
R->P-abs6 & $1.29 \mathrm{E}-17$ & 2.00 & 1.00 \\
R->P-diss1 & $2.36 \mathrm{E}-18$ & 1.75 & 7.79 \\
R->P-diss2 & $1.35 \mathrm{E}-21$ & 2.93 & 6.68 \\
\hline
\end{tabular}




\begin{tabular}{cccc}
\hline 500-950K & $\mathrm{A}\left(\mathrm{cm}^{3}\right.$ molecule $\left.^{-1}\right)$ & $\mathrm{n}$ & $\mathrm{E}_{\mathrm{a}}\left(\mathrm{kcal} \mathrm{mol}^{-1}\right)$ \\
\hline R->P-add1 & $7.38 \mathrm{E}-11$ & -0.18 & -0.25 \\
R->P-add2 & $2.75 \mathrm{E}-12$ & 0.02 & -0.23 \\
R->P-abs1 & $8.42 \mathrm{E}-19$ & 2.34 & 2.00 \\
R->P-abs2 & $7.95 \mathrm{E}-18$ & 2.00 & 1.42 \\
R->P-abs3 & $3.02 \mathrm{E}-18$ & 1.70 & -2.66 \\
R->P-abs3m & $7.13 \mathrm{E}-15$ & 1.03 & 1.67 \\
R->P-abs4 & $2.38 \mathrm{E}-12$ & 0.13 & 0.21 \\
R->P-abs5 & $2.60 \mathrm{E}-15$ & 1.24 & 0.09 \\
R->P-abs6 & $1.91 \mathrm{E}-13$ & 0.68 & 2.40 \\
R->P-diss1 & $1.18 \mathrm{E}-11$ & 0.51 & 14.39 \\
R->P-diss2 & $1.15 \mathrm{E}-18$ & 2.77 & 12.10 \\
\hline
\end{tabular}

2188

\begin{tabular}{cccc}
\hline 1000-2000K & $\mathrm{A}\left(\mathrm{cm}^{3}\right.$ molecule $\left.{ }^{-1}\right)$ & $\mathrm{n}$ & $\mathrm{E}_{\mathrm{a}}\left(\mathrm{kcal} \mathrm{mol}^{-1}\right)$ \\
\hline R-PP-add1 & $9.65 \mathrm{E}-15$ & 0.48 & -8.28 \\
R->P-add2 & $1.75 \mathrm{E}-19$ & 1.91 & -7.30 \\
R->P-abs1 & $1.08 \mathrm{E}-14$ & 1.10 & 3.77 \\
R->P-abs2 & $2.27 \mathrm{E}-13$ & 0.70 & 3.92 \\
R->P-abs3 & $2.00 \mathrm{E}-13$ & 0.35 & 0.76 \\
R->P-abs3m & $1.38 \mathrm{E}-14$ & 0.96 & 1.90 \\
R->P-abs4 & $1.59 \mathrm{E}-14$ & 0.85 & 0.12 \\
R->P-abs5 & $2.87 \mathrm{E}-12$ & 0.35 & 1.86 \\
R->P-abs6 & $1.30 \mathrm{E}-11$ & 0.17 & 3.83 \\
R->P-diss1 & $7.91 \mathrm{E}-17$ & 1.54 & 4.00 \\
R->P-diss2 & $1.10 \mathrm{E}-14$ & 0.94 & 3.91 \\
\hline
\end{tabular}

2189

2190 Table S5.2 The computed RRKM/ME rate coefficients $\left(\mathrm{cm}^{3}\right.$ molecule $\left.{ }^{-1} \mathrm{~s}^{-1}\right)$ for $2-\mathrm{MP}+\mathrm{OH}$ in 2191 the format of $\mathrm{k}=\mathrm{A} \cdot \mathrm{T}^{\mathrm{n}} \cdot \exp \left(-\mathrm{E}_{\mathrm{a}} / \mathrm{RT}\right)$ at $200-1000 \mathrm{~K}, 1 \mathrm{~atm}$.

\begin{tabular}{|c|c|c|c|}
\hline $200-450$ & $\mathrm{~A}\left(\mathrm{~cm}^{3}\right.$ molecule $\left.{ }^{-1}\right)$ & $\mathrm{n}$ & $\mathrm{E}_{\mathrm{a}}\left(\mathrm{kcal} \mathrm{mol}^{-1}\right)$ \\
\hline $\mathrm{R}->\mathrm{P}$-add & $2.94 \mathrm{E}-19$ & 1.86 & 2.99 \\
\hline $\mathrm{R}->\mathrm{P}-\mathrm{abs} 1$ & $2.46 \mathrm{E}-21$ & 2.92 & -2.57 \\
\hline $\mathrm{R}->\mathrm{P}-\mathrm{abs} 2$ & $4.56 \mathrm{E}-12$ & 0.20 & 0.08 \\
\hline $\mathrm{R}->\mathrm{P}-\mathrm{abs} 2 \mathrm{~m}$ & $2.09 \mathrm{E}-19$ & 2.13 & 0.87 \\
\hline $\mathrm{R}->\mathrm{P}-\mathrm{abs} 3$ & $2.37 \mathrm{E}-18$ & 2.00 & -1.83 \\
\hline $\mathrm{R}->\mathrm{P}-\mathrm{abs} 4$ & $1.80 \mathrm{E}-21$ & 3.03 & -2.00 \\
\hline $\mathrm{R}->\mathrm{P}-\mathrm{abs} 5$ & $2.79 \mathrm{E}-21$ & 3.03 & -2.00 \\
\hline R->P-diss 1 & $3.78 \mathrm{E}-22$ & 2.03 & 3.82 \\
\hline $\mathrm{R}->\mathrm{P}$-diss 2 & $6.53 \mathrm{E}-19$ & 1.72 & 2.97 \\
\hline R->RC-abs1(200-240K) & $3.51 \mathrm{E}-15$ & 0.36 & -1.50 \\
\hline R->RC-add(200-230K) & $1.57 \mathrm{E}-16$ & 0.51 & -2.45 \\
\hline
\end{tabular}

2192

\begin{tabular}{|c|c|c|c|}
\hline $500-950$ & $\mathrm{~A}\left(\mathrm{~cm}^{3}\right.$ molecule $\left.{ }^{-1}\right)$ & $\mathrm{n}$ & $\mathrm{E}_{\mathrm{a}}\left(\mathrm{kcal} \mathrm{mol}^{-1}\right)$ \\
\hline R->P-add & $1.55 \mathrm{E}-14$ & 0.06 & 2.29 \\
\hline $\mathrm{R}->\mathrm{P}-\mathrm{abs} 1$ & $3.90 \mathrm{E}-21$ & 2.45 & -4.49 \\
\hline
\end{tabular}




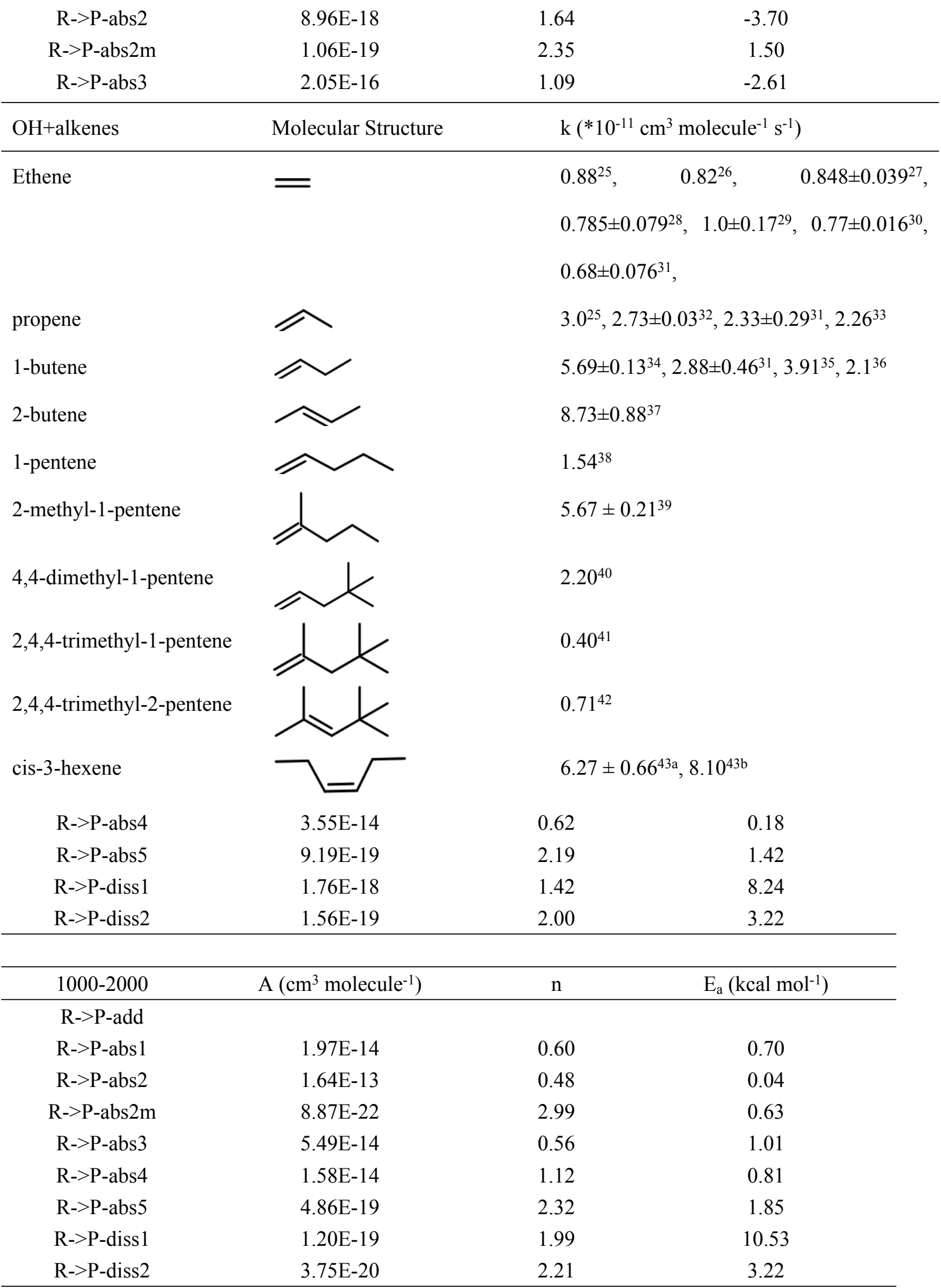

2195 6). Table S6.1 The total rate coefficients based on the previous experiments, theories, and structure-activity relationship (SAR) model under atmospheric conditions 


2-methyl-1-octene

2198 Note:

2199 Relative rate technique (RR) $25,27,30,39,43,45$

2200 Flash photolysis-resonance fluorescence technique (FP-RF) $28-29,34,37$

2201 laser photolysis/laser-induced fluorescence kinetics technique (LP-LIF) $)^{31-32}$

2202 CBS-QB3, TST

2203 BHandHLYP/aug-cc-pVDZ, Microcanonical variational transition state theory (MVT) ${ }^{43 b}$

$2204 \mathrm{CCSD}(\mathrm{T}) / \mathrm{CBS} / / \mathrm{BHandHLYP} / 6-311 \mathrm{G}(\mathrm{d}, \mathrm{p})$, canonical variational transition-state theory $(\mathrm{CVT})^{44}$

$2205 \operatorname{CCSD}(\mathrm{T}) / 6-311++\mathrm{G}(\mathrm{d}, \mathrm{p}) / /$ BHANDHLYP/6-311(d, p), CVT ${ }^{41}$

2206 DLPNO-CCSD(T)/cc-pVTZ//M06-2X/6-311G(d,p), CVT ${ }^{42}$

$2207 \mathrm{UCCSD}(\mathrm{T}) / \mathrm{CBS} / / \mathrm{CASPT} 2 / \mathrm{cc}-\mathrm{pVTZ}$, Conventional transition state theory (cTST) ${ }^{33}$

BHandHLYP/aug-cc-pVDZ, MVT ${ }^{35}$ : s 
2210 M06-2X/aug-cc-pVDZ, CVT $^{38}$

2213 Table S6.2 The total rate coefficients based on the previous experiments and theories for aldehydes $+\mathrm{OH}$ under atmospheric conditions.

\begin{tabular}{|c|c|c|}
\hline $\mathrm{OH}+$ aldehydes & Molecular Structure & $\mathrm{k}\left(* 10^{-11} \mathrm{~cm}^{3}\right.$ molecule $\left.{ }^{-1} \mathrm{~s}^{-1}\right)$ \\
\hline methanal & & $0.92 \pm 0.09^{25}, 91.9^{46 a}, 15.6^{46 b}$ \\
\hline ethanal & & $1.22 \pm 0.27^{47}, 1.44 \pm 0.07^{25}, 1.60 \pm 0.16^{25}$ \\
\hline propanal & & $1.71 \pm 0.24^{47}, 2.1 \pm 0.1^{48}, 1.9 \pm 0.4^{49}, 1.80 \pm$ \\
\hline & & $0.21^{50}, 2.0 \pm 0.3^{51}$ \\
\hline 2-methylpropanal & & $2.42 \pm 0.33^{47}, 2.7 \pm 0.5^{49}, 1.81 \pm 0.21^{50}, 2.58 \pm$ \\
\hline (i-butanal) & & $0.07^{52}, 4.47 \pm 1.39^{53}, 2.60 \pm 0.40^{51}$ \\
\hline 2,2-dimethylpropanal & & $3.06 \pm 0.44^{47}, 2.10 \pm 0.60^{49}, 0.85 \pm 0.10^{50}, 5.16$ \\
\hline (t-pentanal) & & $\pm 2.32^{53}, 2.7 \pm 0.4^{51}$ \\
\hline n-butanal & & $2.06 \pm 0.30^{47}$ \\
\hline 3-methylbutanal & & $2.58 \pm 0.40^{47}$ \\
\hline n-pentanal & & $2.69 \pm 0.39^{47}, 2.6 \pm 0.4^{49}, 1.4 \pm 0.2^{50}, 2.8 \pm 0.2^{51}$ \\
\hline 2-methylpentanal & & $3.32 \pm 0.21^{54}, 2.4$ (this work) \\
\hline 3-methylpentanal & & $2.91 \pm 0.15^{54}$ \\
\hline 4-methylpentanal & & $2.63 \pm 0.11^{54}$ \\
\hline n-hexanal & & $2.86 \pm 0.13^{54}, 3.17 \pm 0.15^{55}, 2.60 \pm 0.21^{56}$ \\
\hline
\end{tabular}


n-heptanal

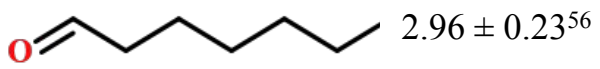

2215

Note:

FP-RF $^{47}$

$\mathrm{RR}^{25,48-52,54-55}$

DF-RF $^{53}$

LP-LIF ${ }^{56}$

$\operatorname{CCSD}(\mathrm{T}) / 6-311++\mathrm{G}(\mathrm{d}, \mathrm{p}) / / \mathrm{MP} 2(\mathrm{FC}) / 6-311++\mathrm{G}(\mathrm{d}, \mathrm{p}), \mathrm{CTST}^{46 a}$

$\operatorname{CCSD}(\mathrm{T}) / 6-311++\mathrm{G}(\mathrm{d}, \mathrm{p}) / / \mathrm{BHandHLYP} / 6-311++\mathrm{G}(\mathrm{d}, \mathrm{p}), \mathrm{CTST}^{46 \mathrm{~b}}$

To further evaluate our computed results, we estimated the total rate coefficients and the yields of different products using the structure-activity relationship (SAR) developed by Atkinson and his co-workers. ${ }^{57-59}$ The rate coefficients based on the SAR model are obtained by summing those of primary $\left(-\mathrm{CH}_{3}\right)$, secondary $\left(-\mathrm{CH}_{2}-\right)$, and tertiary $(-\mathrm{CH}<)$ group, and can be corrected by different substituent factors. According to their results, the total rate coefficients of most of the 290 organic compounds with $\mathrm{OH}$ derived from SAR are in good agreement with the experiment values. The total rate coefficients at $300 \mathrm{~K}, 1$ atm for $3-\mathrm{MH}+$ $\mathrm{OH}$ and 2-MP $+\mathrm{OH}$ are computed to be 3.2 and $2.5 \times 10^{-11} \mathrm{~cm}^{3}$ molecule ${ }^{-1} \mathrm{~s}^{-1}$ by SAR, within a factor of 2 comparing with our results (5.2 and $2.4 \times 10^{-11} \mathrm{~cm}^{3}$ molecule ${ }^{-1} \mathrm{~s}^{-1}$, respectively). For the branching ratios, the combined yields of abs5/6 and abs4/5 from 3-MH $+\mathrm{OH}$ and 2$\mathrm{MP}+\mathrm{OH}$ are deduced to be $4 \%$ and $5 \%$, which are similar to our calculated results $(5 \%$ and $7 \%$, respectively). The branching ratio of $\mathrm{OH}$-addition for 3-MH $+\mathrm{OH}$ estimated by $\mathrm{SAR}(83 \%)$ is closed to that in this work (79\%), nevertheless, the SAR estimation fails to distinguish different addition sites. The dominant product from $2-\mathrm{MP}+\mathrm{OH}$ is predicted to be abs $1(82 \%)$, which is quite different from theoretical calculations that the main product is abs2 $(57 \%)$. Therefore, SAR does provide a simple way to estimate the total rate coefficients (typically under atmospheric conditions) for such reaction systems, but there is still remarkable uncertainty in predicting the branching ratios on different reaction sites.

7). Table S7.1. Final total rate coefficients $\left(\mathrm{cm}^{3}\right.$ molecule $\left.{ }^{-1} \mathrm{~s}^{-1}\right)$ of $3-\mathrm{MH}+\mathrm{OH}$ at $0.1,0.4,0.7$, $1.0,1.2,2.0,4.0,6.0,8.0,10 \mathrm{~atm}, 200-2000 \mathrm{~K}$ 


\begin{tabular}{|c|c|c|c|c|c|c|c|c|c|c|}
\hline $\mathrm{T}(\mathrm{K})$ & 0.1 & 0.4 & 0.7 & 1 & 1.2 & 2 & 4 & 6 & 8 & 10 \\
\hline 200 & 4.7E-11 & 4.7E-11 & 4.7E-11 & 4.7E-11 & 4.7E-11 & 4.7E-11 & 4.7E-11 & 4.7E-11 & 4.7E-11 & 4.7E-11 \\
\hline 210 & $4.8 \mathrm{E}-11$ & $4.8 \mathrm{E}-11$ & $4.8 \mathrm{E}-11$ & $4.8 \mathrm{E}-11$ & $4.8 \mathrm{E}-11$ & $4.8 \mathrm{E}-11$ & $4.8 \mathrm{E}-11$ & $4.8 \mathrm{E}-11$ & $4.8 \mathrm{E}-11$ & $4.8 \mathrm{E}-11$ \\
\hline 220 & 4.9E-11 & 4.9E-11 & 4.9E-11 & $4.9 \mathrm{E}-11$ & $4.9 \mathrm{E}-11$ & 4.9E-11 & $4.9 \mathrm{E}-11$ & 4.9E-11 & 4.9E-11 & 4.9E-11 \\
\hline 230 & $4.9 \mathrm{E}-11$ & $4.9 \mathrm{E}-11$ & $4.9 \mathrm{E}-11$ & $4.9 \mathrm{E}-11$ & $4.9 \mathrm{E}-11$ & $4.9 \mathrm{E}-11$ & $5.0 \mathrm{E}-11$ & $5.0 \mathrm{E}-11$ & $5.0 \mathrm{E}-11$ & $5.0 \mathrm{E}-11$ \\
\hline 240 & $5.0 \mathrm{E}-11$ & $5.0 \mathrm{E}-11$ & $5.0 \mathrm{E}-11$ & $5.0 \mathrm{E}-11$ & $5.0 \mathrm{E}-11$ & $5.0 \mathrm{E}-11$ & $5.0 \mathrm{E}-11$ & $5.0 \mathrm{E}-11$ & $5.0 \mathrm{E}-11$ & $5.0 \mathrm{E}-11$ \\
\hline 250 & $5.1 \mathrm{E}-11$ & $5.1 \mathrm{E}-11$ & $5.1 \mathrm{E}-11$ & $5.1 \mathrm{E}-11$ & $5.1 \mathrm{E}-11$ & $5.1 \mathrm{E}-11$ & $5.1 \mathrm{E}-11$ & $5.1 \mathrm{E}-11$ & $5.1 \mathrm{E}-11$ & 5.1E-11 \\
\hline 260 & $5.1 \mathrm{E}-11$ & $5.1 \mathrm{E}-11$ & $5.1 \mathrm{E}-11$ & $5.1 \mathrm{E}-11$ & $5.1 \mathrm{E}-11$ & $5.1 \mathrm{E}-11$ & $5.1 \mathrm{E}-11$ & $5.1 \mathrm{E}-11$ & $5.1 \mathrm{E}-11$ & 5.1E-11 \\
\hline 270 & $5.2 \mathrm{E}-11$ & $5.2 \mathrm{E}-11$ & $5.2 \mathrm{E}-11$ & $5.2 \mathrm{E}-11$ & $5.2 \mathrm{E}-11$ & $5.2 \mathrm{E}-11$ & $5.2 \mathrm{E}-11$ & $5.2 \mathrm{E}-11$ & $5.2 \mathrm{E}-11$ & 5.2E-11 \\
\hline 280 & $5.2 \mathrm{E}-11$ & $5.2 \mathrm{E}-11$ & $5.2 \mathrm{E}-11$ & $5.2 \mathrm{E}-11$ & $5.2 \mathrm{E}-11$ & $5.2 \mathrm{E}-11$ & $5.2 \mathrm{E}-11$ & $5.2 \mathrm{E}-11$ & $5.2 \mathrm{E}-11$ & $5.2 \mathrm{E}-11$ \\
\hline 290 & $5.2 \mathrm{E}-11$ & $5.2 \mathrm{E}-11$ & $5.2 \mathrm{E}-11$ & $5.2 \mathrm{E}-11$ & $5.2 \mathrm{E}-11$ & $5.2 \mathrm{E}-11$ & $5.2 \mathrm{E}-11$ & $5.2 \mathrm{E}-11$ & $5.2 \mathrm{E}-11$ & 5.2E-11 \\
\hline 300 & $5.2 \mathrm{E}-11$ & $5.2 \mathrm{E}-11$ & $5.2 \mathrm{E}-11$ & $5.2 \mathrm{E}-11$ & $5.2 \mathrm{E}-11$ & $5.2 \mathrm{E}-11$ & $5.2 \mathrm{E}-11$ & $5.2 \mathrm{E}-11$ & $5.2 \mathrm{E}-11$ & 5.2E-11 \\
\hline 310 & $5.2 \mathrm{E}-11$ & $5.2 \mathrm{E}-11$ & $5.2 \mathrm{E}-11$ & $5.2 \mathrm{E}-11$ & $5.2 \mathrm{E}-11$ & $5.2 \mathrm{E}-11$ & $5.2 \mathrm{E}-11$ & $5.2 \mathrm{E}-11$ & $5.2 \mathrm{E}-11$ & 5.2E-11 \\
\hline 320 & $5.3 \mathrm{E}-11$ & $5.3 \mathrm{E}-11$ & $5.3 \mathrm{E}-11$ & $5.3 \mathrm{E}-11$ & $5.3 \mathrm{E}-11$ & $5.3 \mathrm{E}-11$ & $5.3 \mathrm{E}-11$ & $5.3 \mathrm{E}-11$ & $5.3 \mathrm{E}-11$ & 5.3E-11 \\
\hline 330 & $5.3 \mathrm{E}-11$ & $5.3 \mathrm{E}-11$ & $5.3 \mathrm{E}-11$ & $5.3 \mathrm{E}-11$ & $5.3 \mathrm{E}-11$ & $5.3 \mathrm{E}-11$ & $5.3 \mathrm{E}-11$ & $5.3 \mathrm{E}-11$ & $5.3 \mathrm{E}-11$ & 5.3E-11 \\
\hline 340 & $5.3 \mathrm{E}-11$ & $5.3 \mathrm{E}-11$ & $5.3 \mathrm{E}-11$ & $5.3 \mathrm{E}-11$ & $5.3 \mathrm{E}-11$ & $5.3 \mathrm{E}-11$ & $5.3 \mathrm{E}-11$ & $5.3 \mathrm{E}-11$ & $5.3 \mathrm{E}-11$ & 5.3E-11 \\
\hline 350 & $5.3 \mathrm{E}-11$ & $5.3 \mathrm{E}-11$ & $5.3 \mathrm{E}-11$ & $5.3 \mathrm{E}-11$ & $5.3 \mathrm{E}-11$ & $5.3 \mathrm{E}-11$ & $5.3 \mathrm{E}-11$ & $5.3 \mathrm{E}-11$ & $5.3 \mathrm{E}-11$ & 5.3E-11 \\
\hline 360 & $5.3 \mathrm{E}-11$ & $5.3 \mathrm{E}-11$ & $5.3 \mathrm{E}-11$ & $5.3 \mathrm{E}-11$ & $5.3 \mathrm{E}-11$ & $5.3 \mathrm{E}-11$ & $5.3 \mathrm{E}-11$ & $5.3 \mathrm{E}-11$ & $5.3 \mathrm{E}-11$ & 5.3E-11 \\
\hline 370 & 5.3E-11 & $5.3 \mathrm{E}-11$ & $5.3 \mathrm{E}-11$ & $5.3 \mathrm{E}-11$ & $5.3 \mathrm{E}-11$ & $5.3 \mathrm{E}-11$ & $5.3 \mathrm{E}-11$ & $5.3 \mathrm{E}-11$ & $5.3 \mathrm{E}-11$ & 5.3E-11 \\
\hline 380 & $5.3 \mathrm{E}-11$ & $5.3 \mathrm{E}-11$ & $5.3 \mathrm{E}-11$ & $5.3 \mathrm{E}-11$ & $5.3 \mathrm{E}-11$ & $5.3 \mathrm{E}-11$ & $5.3 \mathrm{E}-11$ & $5.3 \mathrm{E}-11$ & $5.3 \mathrm{E}-11$ & 5.3E-11 \\
\hline 390 & $5.3 \mathrm{E}-11$ & $5.3 \mathrm{E}-11$ & $5.3 \mathrm{E}-11$ & $5.3 \mathrm{E}-11$ & $5.3 \mathrm{E}-11$ & $5.3 \mathrm{E}-11$ & $5.3 \mathrm{E}-11$ & $5.3 \mathrm{E}-11$ & $5.3 \mathrm{E}-11$ & 5.3E-11 \\
\hline 400 & $5.3 \mathrm{E}-11$ & $5.3 \mathrm{E}-11$ & $5.3 \mathrm{E}-11$ & $5.3 \mathrm{E}-11$ & $5.3 \mathrm{E}-11$ & $5.3 \mathrm{E}-11$ & $5.3 \mathrm{E}-11$ & $5.3 \mathrm{E}-11$ & $5.3 \mathrm{E}-11$ & 5.3E-11 \\
\hline 410 & $5.2 \mathrm{E}-11$ & $5.2 \mathrm{E}-11$ & $5.2 \mathrm{E}-11$ & $5.2 \mathrm{E}-11$ & $5.2 \mathrm{E}-11$ & $5.2 \mathrm{E}-11$ & $5.2 \mathrm{E}-11$ & $5.2 \mathrm{E}-11$ & $5.2 \mathrm{E}-11$ & $5.2 \mathrm{E}-11$ \\
\hline 420 & $5.2 \mathrm{E}-11$ & $5.2 \mathrm{E}-11$ & $5.2 \mathrm{E}-11$ & $5.2 \mathrm{E}-11$ & $5.2 \mathrm{E}-11$ & $5.2 \mathrm{E}-11$ & $5.2 \mathrm{E}-11$ & $5.2 \mathrm{E}-11$ & $5.2 \mathrm{E}-11$ & $5.2 \mathrm{E}-11$ \\
\hline 430 & $5.2 \mathrm{E}-11$ & $5.2 \mathrm{E}-11$ & $5.2 \mathrm{E}-11$ & $5.2 \mathrm{E}-11$ & $5.2 \mathrm{E}-11$ & $5.2 \mathrm{E}-11$ & $5.2 \mathrm{E}-11$ & $5.2 \mathrm{E}-11$ & $5.2 \mathrm{E}-11$ & $5.2 \mathrm{E}-11$ \\
\hline 440 & $5.2 \mathrm{E}-11$ & $5.2 \mathrm{E}-11$ & $5.2 \mathrm{E}-11$ & $5.2 \mathrm{E}-11$ & $5.2 \mathrm{E}-11$ & $5.2 \mathrm{E}-11$ & $5.2 \mathrm{E}-11$ & $5.2 \mathrm{E}-11$ & $5.2 \mathrm{E}-11$ & $5.2 \mathrm{E}-11$ \\
\hline 450 & $5.2 \mathrm{E}-11$ & $5.2 \mathrm{E}-11$ & $5.2 \mathrm{E}-11$ & $5.2 \mathrm{E}-11$ & $5.2 \mathrm{E}-11$ & $5.2 \mathrm{E}-11$ & $5.2 \mathrm{E}-11$ & $5.2 \mathrm{E}-11$ & $5.2 \mathrm{E}-11$ & $5.2 \mathrm{E}-11$ \\
\hline 500 & $5.2 \mathrm{E}-11$ & $5.2 \mathrm{E}-11$ & $5.2 \mathrm{E}-11$ & $5.2 \mathrm{E}-11$ & $5.2 \mathrm{E}-11$ & $5.2 \mathrm{E}-11$ & $5.2 \mathrm{E}-11$ & $5.2 \mathrm{E}-11$ & $5.2 \mathrm{E}-11$ & $5.2 \mathrm{E}-11$ \\
\hline 550 & $5.1 \mathrm{E}-11$ & $5.1 \mathrm{E}-11$ & $5.2 \mathrm{E}-11$ & $5.2 \mathrm{E}-11$ & $5.2 \mathrm{E}-11$ & $5.2 \mathrm{E}-11$ & $5.2 \mathrm{E}-11$ & $5.2 \mathrm{E}-11$ & $5.2 \mathrm{E}-11$ & $5.2 \mathrm{E}-11$ \\
\hline 600 & $5.0 \mathrm{E}-11$ & $5.1 \mathrm{E}-11$ & $5.2 \mathrm{E}-11$ & $5.2 \mathrm{E}-11$ & $5.2 \mathrm{E}-11$ & $5.2 \mathrm{E}-11$ & $5.2 \mathrm{E}-11$ & $5.2 \mathrm{E}-11$ & $5.2 \mathrm{E}-11$ & $5.2 \mathrm{E}-11$ \\
\hline 650 & $5.0 \mathrm{E}-11$ & $5.2 \mathrm{E}-11$ & $5.2 \mathrm{E}-11$ & $5.2 \mathrm{E}-11$ & $5.2 \mathrm{E}-11$ & $5.2 \mathrm{E}-11$ & $5.3 \mathrm{E}-11$ & $5.3 \mathrm{E}-11$ & $5.3 \mathrm{E}-11$ & $5.3 \mathrm{E}-11$ \\
\hline 700 & $4.9 \mathrm{E}-11$ & $5.2 \mathrm{E}-11$ & $5.3 \mathrm{E}-11$ & $5.3 \mathrm{E}-11$ & $5.3 \mathrm{E}-11$ & $5.3 \mathrm{E}-11$ & $5.4 \mathrm{E}-11$ & $5.4 \mathrm{E}-11$ & $5.4 \mathrm{E}-11$ & $5.4 \mathrm{E}-11$ \\
\hline 750 & 4.7E-11 & $5.2 \mathrm{E}-11$ & $5.3 \mathrm{E}-11$ & $5.4 \mathrm{E}-11$ & $5.4 \mathrm{E}-11$ & $5.5 \mathrm{E}-11$ & $5.5 \mathrm{E}-11$ & $5.5 \mathrm{E}-11$ & $5.5 \mathrm{E}-11$ & $5.5 \mathrm{E}-11$ \\
\hline 800 & 4.6E-11 & $5.2 \mathrm{E}-11$ & $5.4 \mathrm{E}-11$ & $5.5 \mathrm{E}-11$ & $5.5 \mathrm{E}-11$ & $5.6 \mathrm{E}-11$ & $5.7 \mathrm{E}-11$ & $5.7 \mathrm{E}-11$ & $5.7 \mathrm{E}-11$ & $5.7 \mathrm{E}-11$ \\
\hline 850 & $4.5 \mathrm{E}-11$ & $5.2 \mathrm{E}-11$ & $5.4 \mathrm{E}-11$ & $5.6 \mathrm{E}-11$ & $5.6 \mathrm{E}-11$ & 5.7E-11 & $5.9 \mathrm{E}-11$ & $5.9 \mathrm{E}-11$ & $5.9 \mathrm{E}-11$ & 5.9E-11 \\
\hline 900 & 4.4E-11 & $5.2 \mathrm{E}-11$ & $5.5 \mathrm{E}-11$ & $5.6 \mathrm{E}-11$ & $5.7 \mathrm{E}-11$ & $5.9 \mathrm{E}-11$ & $6.0 \mathrm{E}-11$ & $6.1 \mathrm{E}-11$ & $6.1 \mathrm{E}-11$ & $6.2 \mathrm{E}-11$ \\
\hline 950 & $4.5 \mathrm{E}-11$ & $5.2 \mathrm{E}-11$ & $5.5 \mathrm{E}-11$ & $5.7 \mathrm{E}-11$ & $5.8 \mathrm{E}-11$ & $6.0 \mathrm{E}-11$ & $6.2 \mathrm{E}-11$ & $6.3 \mathrm{E}-11$ & $6.4 \mathrm{E}-11$ & $6.4 \mathrm{E}-11$ \\
\hline 1000 & $4.5 \mathrm{E}-11$ & $5.2 \mathrm{E}-11$ & $5.6 \mathrm{E}-11$ & $5.8 \mathrm{E}-11$ & $5.8 \mathrm{E}-11$ & $6.1 \mathrm{E}-11$ & $6.4 \mathrm{E}-11$ & $6.5 \mathrm{E}-11$ & $6.6 \mathrm{E}-11$ & $6.7 \mathrm{E}-11$ \\
\hline 1100 & $4.5 \mathrm{E}-11$ & $5.4 \mathrm{E}-11$ & $5.7 \mathrm{E}-11$ & $5.9 \mathrm{E}-11$ & $6.0 \mathrm{E}-11$ & $6.3 \mathrm{E}-11$ & $6.7 \mathrm{E}-11$ & $6.9 \mathrm{E}-11$ & $7.1 \mathrm{E}-11$ & 7.1E-11 \\
\hline 1200 & 4.9E-11 & $5.2 \mathrm{E}-11$ & $5.3 \mathrm{E}-11$ & $6.2 \mathrm{E}-11$ & $6.3 \mathrm{E}-11$ & $6.6 \mathrm{E}-11$ & $7.1 \mathrm{E}-11$ & 7.3E-11 & $7.5 \mathrm{E}-11$ & $7.6 \mathrm{E}-11$ \\
\hline 1300 & $5.6 \mathrm{E}-11$ & $5.8 \mathrm{E}-11$ & $5.9 \mathrm{E}-11$ & $5.9 \mathrm{E}-11$ & $5.9 \mathrm{E}-11$ & $6.0 \mathrm{E}-11$ & 7.4E-11 & 7.7E-11 & 7.9E-11 & $8.0 \mathrm{E}-11$ \\
\hline 1400 & $6.3 \mathrm{E}-11$ & $6.3 \mathrm{E}-11$ & $6.3 \mathrm{E}-11$ & $6.6 \mathrm{E}-11$ & $6.6 \mathrm{E}-11$ & $6.7 \mathrm{E}-11$ & $6.7 \mathrm{E}-11$ & $8.1 \mathrm{E}-11$ & 8.3E-11 & $8.5 \mathrm{E}-11$ \\
\hline 1500 & $7.1 \mathrm{E}-11$ & $7.1 \mathrm{E}-11$ & $7.1 \mathrm{E}-11$ & $7.1 \mathrm{E}-11$ & $7.1 \mathrm{E}-11$ & $7.3 \mathrm{E}-11$ & $7.4 \mathrm{E}-11$ & $7.5 \mathrm{E}-11$ & $7.5 \mathrm{E}-11$ & $8.9 \mathrm{E}-11$ \\
\hline 1600 & $7.8 \mathrm{E}-11$ & $7.8 \mathrm{E}-11$ & $7.8 \mathrm{E}-11$ & $7.8 \mathrm{E}-11$ & $7.8 \mathrm{E}-11$ & $7.8 \mathrm{E}-11$ & $8.1 \mathrm{E}-11$ & $8.1 \mathrm{E}-11$ & $8.2 \mathrm{E}-11$ & $8.2 \mathrm{E}-11$ \\
\hline 1700 & $8.5 \mathrm{E}-11$ & $8.5 \mathrm{E}-11$ & $8.5 \mathrm{E}-11$ & $8.5 \mathrm{E}-11$ & $8.5 \mathrm{E}-11$ & $8.5 \mathrm{E}-11$ & $8.5 \mathrm{E}-11$ & $8.8 \mathrm{E}-11$ & $8.8 \mathrm{E}-11$ & 8.9E-11 \\
\hline
\end{tabular}


$1800 \quad 9.1 \mathrm{E}-11 \quad 9.1 \mathrm{E}-11 \quad 9.1 \mathrm{E}-11 \quad 9.1 \mathrm{E}-11 \quad 9.1 \mathrm{E}-11 \quad 9.1 \mathrm{E}-11 \quad 9.1 \mathrm{E}-11 \quad 9.1 \mathrm{E}-11 \quad 9.1 \mathrm{E}-11 \quad 9.5 \mathrm{E}-11$

$1900 \quad 9.8 \mathrm{E}-11 \quad 9.8 \mathrm{E}-11 \quad 9.8 \mathrm{E}-11 \quad 9.8 \mathrm{E}-11 \quad 9.8 \mathrm{E}-11 \quad 9.8 \mathrm{E}-11 \quad 9.8 \mathrm{E}-11 \quad 9.8 \mathrm{E}-11 \quad 9.8 \mathrm{E}-11 \quad 9.8 \mathrm{E}-11$

$\begin{array}{lllllllllll}2000 & 1.0 \mathrm{E}-10 & 1.0 \mathrm{E}-10 & 1.0 \mathrm{E}-10 & 1.0 \mathrm{E}-10 & 1.0 \mathrm{E}-10 & 1.0 \mathrm{E}-10 & 1.0 \mathrm{E}-10 & 1.0 \mathrm{E}-10 & 1.0 \mathrm{E}-10 & 1.0 \mathrm{E}-10\end{array}$

2245 Table S7.2. Final total rate coefficients $\left(\mathrm{cm}^{3}\right.$ molecule $\left.\mathrm{e}^{-1} \mathrm{~s}^{-1}\right)$ of $2-\mathrm{MP}+\mathrm{OH}$ at $0.1,0.4,0.7,1.0$,

$2246 \quad 1.2,2.0,4.0,6.0,8.0,10 \mathrm{~atm}, 200-2000 \mathrm{~K}$

\begin{tabular}{|c|c|c|c|c|c|c|c|c|c|c|}
\hline $\mathrm{T}(\mathrm{K})$ & 0.1 & 0.4 & 0.7 & 1 & 1.2 & 2 & 4 & 6 & 8 & 10 \\
\hline 200 & $3.1 \mathrm{E}-11$ & $3.2 \mathrm{E}-11$ & $3.3 \mathrm{E}-11$ & $3.3 \mathrm{E}-11$ & $3.4 \mathrm{E}-11$ & $3.5 \mathrm{E}-11$ & $3.6 \mathrm{E}-11$ & $3.6 \mathrm{E}-11$ & $3.7 \mathrm{E}-11$ & $3.8 \mathrm{E}-11$ \\
\hline 210 & $3.1 \mathrm{E}-11$ & $3.1 \mathrm{E}-11$ & $3.2 \mathrm{E}-11$ & $3.2 \mathrm{E}-11$ & $3.2 \mathrm{E}-11$ & $3.3 \mathrm{E}-11$ & $3.4 \mathrm{E}-11$ & $3.5 \mathrm{E}-11$ & $3.6 \mathrm{E}-11$ & $3.6 \mathrm{E}-11$ \\
\hline 220 & $3.0 \mathrm{E}-11$ & $3.0 \mathrm{E}-11$ & $3.1 \mathrm{E}-11$ & $3.1 \mathrm{E}-11$ & $3.1 \mathrm{E}-11$ & $3.2 \mathrm{E}-11$ & $3.3 \mathrm{E}-11$ & $3.4 \mathrm{E}-11$ & $3.4 \mathrm{E}-11$ & $3.5 \mathrm{E}-11$ \\
\hline 230 & $2.9 \mathrm{E}-11$ & $2.9 \mathrm{E}-11$ & $3.0 \mathrm{E}-11$ & $3.0 \mathrm{E}-11$ & $3.0 \mathrm{E}-11$ & $3.1 \mathrm{E}-11$ & $3.2 \mathrm{E}-11$ & $3.2 \mathrm{E}-11$ & 3.3E-11 & 3.4E-11 \\
\hline 240 & $2.8 \mathrm{E}-11$ & $2.8 \mathrm{E}-11$ & $2.9 \mathrm{E}-11$ & $2.9 \mathrm{E}-11$ & $2.9 \mathrm{E}-11$ & $3.0 \mathrm{E}-11$ & $3.1 \mathrm{E}-11$ & $3.1 \mathrm{E}-11$ & $3.2 \mathrm{E}-11$ & $3.2 \mathrm{E}-11$ \\
\hline 250 & $2.8 \mathrm{E}-11$ & $2.8 \mathrm{E}-11$ & $2.8 \mathrm{E}-11$ & $2.8 \mathrm{E}-11$ & $2.8 \mathrm{E}-11$ & $2.8 \mathrm{E}-11$ & $3.0 \mathrm{E}-11$ & $3.0 \mathrm{E}-11$ & $3.0 \mathrm{E}-11$ & $3.1 \mathrm{E}-11$ \\
\hline 260 & $2.7 \mathrm{E}-11$ & $2.7 \mathrm{E}-11$ & $2.7 \mathrm{E}-11$ & $2.7 \mathrm{E}-11$ & $2.7 \mathrm{E}-11$ & $2.7 \mathrm{E}-11$ & $2.8 \mathrm{E}-11$ & $2.9 \mathrm{E}-11$ & $2.9 \mathrm{E}-11$ & $3.0 \mathrm{E}-11$ \\
\hline 270 & $2.6 \mathrm{E}-11$ & $2.6 \mathrm{E}-11$ & $2.6 \mathrm{E}-11$ & $2.6 \mathrm{E}-11$ & $2.6 \mathrm{E}-11$ & $2.7 \mathrm{E}-11$ & $2.7 \mathrm{E}-11$ & $2.8 \mathrm{E}-11$ & $2.9 \mathrm{E}-11$ & $2.9 \mathrm{E}-11$ \\
\hline 280 & $2.5 \mathrm{E}-11$ & $2.6 \mathrm{E}-11$ & $2.6 \mathrm{E}-11$ & $2.6 \mathrm{E}-11$ & $2.6 \mathrm{E}-11$ & $2.6 \mathrm{E}-11$ & $2.6 \mathrm{E}-11$ & 2.7E-11 & $2.8 \mathrm{E}-11$ & $2.8 \mathrm{E}-11$ \\
\hline 290 & $2.5 \mathrm{E}-11$ & $2.5 \mathrm{E}-11$ & $2.5 \mathrm{E}-11$ & $2.5 \mathrm{E}-11$ & $2.5 \mathrm{E}-11$ & $2.5 \mathrm{E}-11$ & $2.5 \mathrm{E}-11$ & $2.6 \mathrm{E}-11$ & $2.6 \mathrm{E}-11$ & 2.7E-11 \\
\hline 300 & 2.4E-11 & $2.4 \mathrm{E}-11$ & 2.4E-11 & 2.4E-11 & 2.4E-11 & $2.5 \mathrm{E}-11$ & $2.5 \mathrm{E}-11$ & $2.5 \mathrm{E}-11$ & $2.5 \mathrm{E}-11$ & $2.5 \mathrm{E}-11$ \\
\hline 310 & $2.4 \mathrm{E}-11$ & $2.4 \mathrm{E}-11$ & $2.4 \mathrm{E}-11$ & $2.4 \mathrm{E}-11$ & $2.4 \mathrm{E}-11$ & $2.4 \mathrm{E}-11$ & $2.4 \mathrm{E}-11$ & $2.4 \mathrm{E}-11$ & $2.4 \mathrm{E}-11$ & $2.4 \mathrm{E}-11$ \\
\hline 320 & $2.3 \mathrm{E}-11$ & $2.3 \mathrm{E}-11$ & 2.3E-11 & 2.3E-11 & 2.3E-11 & 2.3E-11 & 2.3E-11 & $2.3 \mathrm{E}-11$ & 2.4E-11 & 2.4E-11 \\
\hline 330 & $2.2 \mathrm{E}-11$ & $2.2 \mathrm{E}-11$ & $2.2 \mathrm{E}-11$ & $2.2 \mathrm{E}-11$ & $2.2 \mathrm{E}-11$ & $2.3 \mathrm{E}-11$ & $2.3 \mathrm{E}-11$ & $2.3 \mathrm{E}-11$ & $2.3 \mathrm{E}-11$ & $2.3 \mathrm{E}-11$ \\
\hline 340 & $2.2 \mathrm{E}-11$ & $2.2 \mathrm{E}-11$ & $2.2 \mathrm{E}-11$ & 2.2E-11 & $2.2 \mathrm{E}-11$ & $2.2 \mathrm{E}-11$ & $2.2 \mathrm{E}-11$ & 2.2E-11 & $2.2 \mathrm{E}-11$ & $2.2 \mathrm{E}-11$ \\
\hline 350 & $2.1 \mathrm{E}-11$ & $2.1 \mathrm{E}-11$ & $2.1 \mathrm{E}-11$ & $2.1 \mathrm{E}-11$ & $2.1 \mathrm{E}-11$ & $2.1 \mathrm{E}-11$ & $2.1 \mathrm{E}-11$ & $2.2 \mathrm{E}-11$ & 2.2E-11 & $2.2 \mathrm{E}-11$ \\
\hline 360 & $2.1 \mathrm{E}-11$ & $2.1 \mathrm{E}-11$ & $2.1 \mathrm{E}-11$ & $2.1 \mathrm{E}-11$ & $2.1 \mathrm{E}-11$ & $2.1 \mathrm{E}-11$ & $2.1 \mathrm{E}-11$ & $2.1 \mathrm{E}-11$ & $2.1 \mathrm{E}-11$ & $2.1 \mathrm{E}-11$ \\
\hline 370 & $2.0 \mathrm{E}-11$ & $2.0 \mathrm{E}-11$ & $2.0 \mathrm{E}-11$ & $2.0 \mathrm{E}-11$ & $2.0 \mathrm{E}-11$ & $2.0 \mathrm{E}-11$ & $2.0 \mathrm{E}-11$ & $2.0 \mathrm{E}-11$ & $2.0 \mathrm{E}-11$ & $2.0 \mathrm{E}-11$ \\
\hline 380 & $2.0 \mathrm{E}-11$ & $2.0 \mathrm{E}-11$ & $2.0 \mathrm{E}-11$ & $2.0 \mathrm{E}-11$ & $2.0 \mathrm{E}-11$ & $2.0 \mathrm{E}-11$ & $2.0 \mathrm{E}-11$ & $2.0 \mathrm{E}-11$ & $2.0 \mathrm{E}-11$ & $2.0 \mathrm{E}-11$ \\
\hline 390 & $1.9 \mathrm{E}-11$ & $1.9 \mathrm{E}-11$ & $1.9 \mathrm{E}-11$ & $1.9 \mathrm{E}-11$ & $1.9 \mathrm{E}-11$ & $1.9 \mathrm{E}-11$ & $1.9 \mathrm{E}-11$ & $1.9 \mathrm{E}-11$ & $1.9 \mathrm{E}-11$ & $1.9 \mathrm{E}-11$ \\
\hline 400 & $1.9 \mathrm{E}-11$ & $1.9 \mathrm{E}-11$ & $1.9 \mathrm{E}-11$ & $1.9 \mathrm{E}-11$ & $1.9 \mathrm{E}-11$ & $1.9 \mathrm{E}-11$ & $1.9 \mathrm{E}-11$ & $1.9 \mathrm{E}-11$ & $1.9 \mathrm{E}-11$ & $1.9 \mathrm{E}-11$ \\
\hline 410 & $1.8 \mathrm{E}-11$ & $1.8 \mathrm{E}-11$ & $1.8 \mathrm{E}-11$ & $1.8 \mathrm{E}-11$ & $1.8 \mathrm{E}-11$ & $1.8 \mathrm{E}-11$ & $1.8 \mathrm{E}-11$ & $1.8 \mathrm{E}-11$ & $1.8 \mathrm{E}-11$ & $1.8 \mathrm{E}-11$ \\
\hline 420 & $1.8 \mathrm{E}-11$ & $1.8 \mathrm{E}-11$ & $1.8 \mathrm{E}-11$ & $1.8 \mathrm{E}-11$ & $1.8 \mathrm{E}-11$ & $1.8 \mathrm{E}-11$ & $1.8 \mathrm{E}-11$ & $1.8 \mathrm{E}-11$ & $1.8 \mathrm{E}-11$ & $1.8 \mathrm{E}-11$ \\
\hline 430 & $1.7 \mathrm{E}-11$ & $1.7 \mathrm{E}-11$ & $1.7 \mathrm{E}-11$ & $1.7 \mathrm{E}-11$ & $1.7 \mathrm{E}-11$ & $1.7 \mathrm{E}-11$ & $1.7 \mathrm{E}-11$ & $1.7 \mathrm{E}-11$ & $1.7 \mathrm{E}-11$ & $1.7 \mathrm{E}-11$ \\
\hline 440 & $1.7 \mathrm{E}-11$ & $1.7 \mathrm{E}-11$ & $1.7 \mathrm{E}-11$ & 1.7E-11 & $1.7 \mathrm{E}-11$ & $1.7 \mathrm{E}-11$ & $1.7 \mathrm{E}-11$ & $1.7 \mathrm{E}-11$ & $1.7 \mathrm{E}-11$ & $1.7 \mathrm{E}-11$ \\
\hline 450 & $1.7 \mathrm{E}-11$ & $1.7 \mathrm{E}-11$ & $1.7 \mathrm{E}-11$ & $1.7 \mathrm{E}-11$ & $1.7 \mathrm{E}-11$ & $1.7 \mathrm{E}-11$ & $1.7 \mathrm{E}-11$ & $1.7 \mathrm{E}-11$ & $1.7 \mathrm{E}-11$ & $1.7 \mathrm{E}-11$ \\
\hline 500 & $1.5 \mathrm{E}-11$ & $1.5 \mathrm{E}-11$ & $1.5 \mathrm{E}-11$ & $1.5 \mathrm{E}-11$ & $1.5 \mathrm{E}-11$ & $1.5 \mathrm{E}-11$ & $1.5 \mathrm{E}-11$ & $1.5 \mathrm{E}-11$ & $1.5 \mathrm{E}-11$ & $1.5 \mathrm{E}-11$ \\
\hline 550 & $1.4 \mathrm{E}-11$ & $1.4 \mathrm{E}-11$ & $1.4 \mathrm{E}-11$ & $1.4 \mathrm{E}-11$ & $1.4 \mathrm{E}-11$ & $1.4 \mathrm{E}-11$ & $1.4 \mathrm{E}-11$ & $1.4 \mathrm{E}-11$ & $1.4 \mathrm{E}-11$ & $1.4 \mathrm{E}-11$ \\
\hline 600 & $1.3 \mathrm{E}-11$ & $1.3 \mathrm{E}-11$ & $1.3 \mathrm{E}-11$ & $1.3 \mathrm{E}-11$ & $1.3 \mathrm{E}-11$ & $1.3 \mathrm{E}-11$ & $1.3 \mathrm{E}-11$ & $1.3 \mathrm{E}-11$ & $1.3 \mathrm{E}-11$ & $1.3 \mathrm{E}-11$ \\
\hline 650 & $1.2 \mathrm{E}-11$ & $1.2 \mathrm{E}-11$ & $1.2 \mathrm{E}-11$ & $1.2 \mathrm{E}-11$ & $1.2 \mathrm{E}-11$ & $1.2 \mathrm{E}-11$ & $1.2 \mathrm{E}-11$ & $1.2 \mathrm{E}-11$ & $1.2 \mathrm{E}-11$ & $1.2 \mathrm{E}-11$ \\
\hline 700 & $1.2 \mathrm{E}-11$ & $1.2 \mathrm{E}-11$ & $1.2 \mathrm{E}-11$ & $1.2 \mathrm{E}-11$ & $1.2 \mathrm{E}-11$ & $1.2 \mathrm{E}-11$ & $1.2 \mathrm{E}-11$ & $1.2 \mathrm{E}-11$ & $1.2 \mathrm{E}-11$ & $1.2 \mathrm{E}-11$ \\
\hline 750 & $1.1 \mathrm{E}-11$ & $1.1 \mathrm{E}-11$ & $1.1 \mathrm{E}-11$ & $1.1 \mathrm{E}-11$ & $1.1 \mathrm{E}-11$ & $1.1 \mathrm{E}-11$ & $1.1 \mathrm{E}-11$ & $1.1 \mathrm{E}-11$ & $1.1 \mathrm{E}-11$ & $1.1 \mathrm{E}-11$ \\
\hline 800 & $1.1 \mathrm{E}-11$ & $1.1 \mathrm{E}-11$ & $1.1 \mathrm{E}-11$ & $1.1 \mathrm{E}-11$ & $1.1 \mathrm{E}-11$ & $1.1 \mathrm{E}-11$ & $1.1 \mathrm{E}-11$ & $1.1 \mathrm{E}-11$ & $1.1 \mathrm{E}-11$ & $1.1 \mathrm{E}-11$ \\
\hline 850 & $1.1 \mathrm{E}-11$ & $1.1 \mathrm{E}-11$ & $1.1 \mathrm{E}-11$ & $1.1 \mathrm{E}-11$ & $1.1 \mathrm{E}-11$ & $1.1 \mathrm{E}-11$ & $1.1 \mathrm{E}-11$ & $1.1 \mathrm{E}-11$ & $1.1 \mathrm{E}-11$ & $1.1 \mathrm{E}-11$ \\
\hline 900 & $1.2 \mathrm{E}-11$ & $1.2 \mathrm{E}-11$ & $1.2 \mathrm{E}-11$ & $1.2 \mathrm{E}-11$ & $1.2 \mathrm{E}-11$ & $1.2 \mathrm{E}-11$ & $1.2 \mathrm{E}-11$ & $1.2 \mathrm{E}-11$ & $1.2 \mathrm{E}-11$ & $1.2 \mathrm{E}-11$ \\
\hline 950 & $1.2 \mathrm{E}-11$ & $1.2 \mathrm{E}-11$ & $1.2 \mathrm{E}-11$ & $1.2 \mathrm{E}-11$ & $1.2 \mathrm{E}-11$ & $1.2 \mathrm{E}-11$ & $1.2 \mathrm{E}-11$ & $1.2 \mathrm{E}-11$ & $1.2 \mathrm{E}-11$ & $1.2 \mathrm{E}-11$ \\
\hline \multirow[t]{2}{*}{1000} & $1.2 \mathrm{E}-11$ & $1.2 \mathrm{E}-11$ & $1.2 \mathrm{E}-11$ & $1.2 \mathrm{E}-11$ & $1.2 \mathrm{E}-11$ & $1.2 \mathrm{E}-11$ & $1.2 \mathrm{E}-11$ & $1.2 \mathrm{E}-11$ & $1.2 \mathrm{E}-11$ & $1.2 \mathrm{E}-11$ \\
\hline & & & & & S-64 & & & & & \\
\hline
\end{tabular}




\begin{tabular}{lllllllllll}
1100 & $1.3 \mathrm{E}-11$ & $1.3 \mathrm{E}-11$ & $1.3 \mathrm{E}-11$ & $1.3 \mathrm{E}-11$ & $1.3 \mathrm{E}-11$ & $1.3 \mathrm{E}-11$ & $1.3 \mathrm{E}-11$ & $1.3 \mathrm{E}-11$ & $1.3 \mathrm{E}-11$ & $1.3 \mathrm{E}-11$ \\
1200 & $1.5 \mathrm{E}-11$ & $1.5 \mathrm{E}-11$ & $1.5 \mathrm{E}-11$ & $1.5 \mathrm{E}-11$ & $1.5 \mathrm{E}-11$ & $1.5 \mathrm{E}-11$ & $1.5 \mathrm{E}-11$ & $1.5 \mathrm{E}-11$ & $1.5 \mathrm{E}-11$ & $1.5 \mathrm{E}-11$ \\
1300 & $1.7 \mathrm{E}-11$ & $1.7 \mathrm{E}-11$ & $1.7 \mathrm{E}-11$ & $1.7 \mathrm{E}-11$ & $1.7 \mathrm{E}-11$ & $1.7 \mathrm{E}-11$ & $1.7 \mathrm{E}-11$ & $1.7 \mathrm{E}-11$ & $1.7 \mathrm{E}-11$ & $1.7 \mathrm{E}-11$ \\
1400 & $1.9 \mathrm{E}-11$ & $1.9 \mathrm{E}-11$ & $1.9 \mathrm{E}-11$ & $1.9 \mathrm{E}-11$ & $1.9 \mathrm{E}-11$ & $1.9 \mathrm{E}-11$ & $1.9 \mathrm{E}-11$ & $1.9 \mathrm{E}-11$ & $1.9 \mathrm{E}-11$ & $1.9 \mathrm{E}-11$ \\
1500 & $2.1 \mathrm{E}-11$ & $2.1 \mathrm{E}-11$ & $2.1 \mathrm{E}-11$ & $2.1 \mathrm{E}-11$ & $2.1 \mathrm{E}-11$ & $2.1 \mathrm{E}-11$ & $2.1 \mathrm{E}-11$ & $2.1 \mathrm{E}-11$ & $2.1 \mathrm{E}-11$ & $2.1 \mathrm{E}-11$ \\
1600 & $2.4 \mathrm{E}-11$ & $2.4 \mathrm{E}-11$ & $2.4 \mathrm{E}-11$ & $2.4 \mathrm{E}-11$ & $2.4 \mathrm{E}-11$ & $2.4 \mathrm{E}-11$ & $2.4 \mathrm{E}-11$ & $2.4 \mathrm{E}-11$ & $2.4 \mathrm{E}-11$ & $2.4 \mathrm{E}-11$ \\
1700 & $2.7 \mathrm{E}-11$ & $2.7 \mathrm{E}-11$ & $2.7 \mathrm{E}-11$ & $2.7 \mathrm{E}-11$ & $2.7 \mathrm{E}-11$ & $2.7 \mathrm{E}-11$ & $2.7 \mathrm{E}-11$ & $2.7 \mathrm{E}-11$ & $2.7 \mathrm{E}-11$ & $2.7 \mathrm{E}-11$ \\
1800 & $3.0 \mathrm{E}-11$ & $3.0 \mathrm{E}-11$ & $3.0 \mathrm{E}-11$ & $3.0 \mathrm{E}-11$ & $3.0 \mathrm{E}-11$ & $3.0 \mathrm{E}-11$ & $3.0 \mathrm{E}-11$ & $3.0 \mathrm{E}-11$ & $3.0 \mathrm{E}-11$ & $3.0 \mathrm{E}-11$ \\
1900 & $3.4 \mathrm{E}-11$ & $3.4 \mathrm{E}-11$ & $3.4 \mathrm{E}-11$ & $3.4 \mathrm{E}-11$ & $3.4 \mathrm{E}-11$ & $3.4 \mathrm{E}-11$ & $3.4 \mathrm{E}-11$ & $3.4 \mathrm{E}-11$ & $3.4 \mathrm{E}-11$ & $3.4 \mathrm{E}-11$ \\
2000 & $3.8 \mathrm{E}-11$ & $3.8 \mathrm{E}-11$ & $3.8 \mathrm{E}-11$ & $3.8 \mathrm{E}-11$ & $3.8 \mathrm{E}-11$ & $3.8 \mathrm{E}-11$ & $3.8 \mathrm{E}-11$ & $3.8 \mathrm{E}-11$ & $3.8 \mathrm{E}-11$ & $3.8 \mathrm{E}-11$ \\
\hline
\end{tabular}

2247

2248

2249

2250

2251

2252

2253

2254

2255

2256

2257

2258

2259

2260

2261

2262

2263

2264

2265

2266

2267

For 3-MH $+\mathrm{OH}$, there are two crossover temperatures for the pressure dependence between 200-2000 K, which correspond to the ranges of 200-380 K, 380-1100 K, and 1100-2000 K. The maximum variation of the total rate coefficient of $3-\mathrm{MH}+\mathrm{OH}$ at $1100 \mathrm{~K}$ is $59.6 \%$ at the temperature (200-2000K) and pressure (0.1-10atm). The pressure dependence effect is much weaker at low temperature, reaching an increasing factor of $0.2 \%$ at $200 \mathrm{~K}$. The pressure dependence of H-abstraction in Figure S5 has a negligible impact at 200-2000K, 0.1-10atm. With the increase of temperature, the pressure-dependent effect of addition reaction becomes more and more apparent, which can be increased by 15.1 times at $1100 \mathrm{~K}$. On the contrary, the negative pressure dependent effect for the rate coefficients of addition-dissociation reaction decrease with increasing temperature.

For 2-MP $+\mathrm{OH}$, the pressure dependence for the total rate coefficients is insignificant, with a maximum factor of 1.2 at $200 \mathrm{~K}$. The rate coefficient of the $2-\mathrm{MP}+\mathrm{OH}$ addition reaction channel has a similar variation trend as that of the $3-\mathrm{MH}$, reaching the maximum factor of 7.3 at $850 \mathrm{~K}$. The addition-dissociation and the main $\mathrm{H}$-abstraction pathways of 2-MP are insignificant as temperature and pressure change in Figure S3. The $\mathrm{R} \rightarrow \mathrm{abs} 1$ channel shows a slight negative pressure dependence with a maximum factor of 0.51 at $200 \mathrm{~K}$. Conversely, a slight positive pressure dependence effect was also present in the $\mathrm{R} \rightarrow \mathrm{RCabs} 1$ channel.

8) Figure S1.1 The branching ratios of various reaction channels for $3-\mathrm{MH}+\mathrm{OH}$ at $1.0 \mathrm{~atm}$, $200-2000 \mathrm{~K}$ 


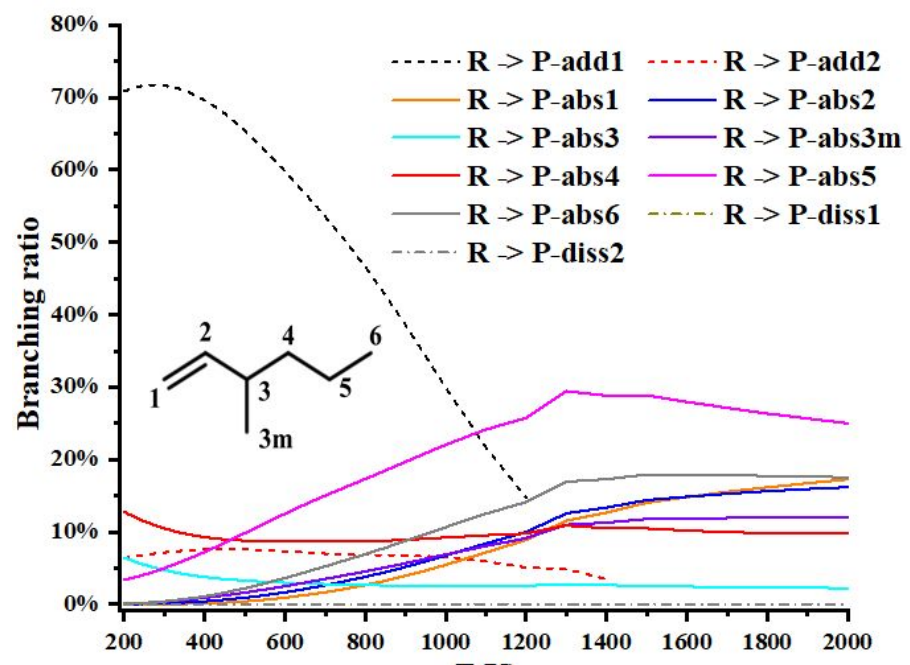

2269 Figure S1.2 The branching ratios of various reaction channels for 2-MP $+\mathrm{OH}$ at $1.0 \mathrm{~atm}, 200$ $2000 \mathrm{~K}$

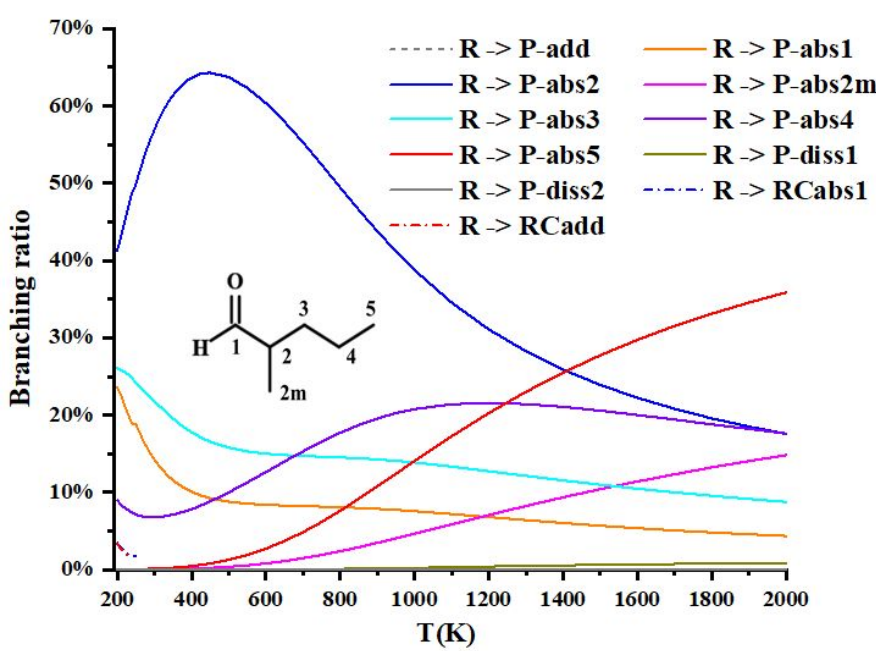

2273 9). Figure S2.1. Final total rate coefficients $\left(\mathrm{cm}^{3}\right.$ molecule $\left.\mathrm{e}^{-1} \mathrm{~s}^{-1}\right)$ of $3-\mathrm{MH}+\mathrm{OH}$ at $0.1,0.4,0.7$, $2274 \quad 1.0,1.2,2.0,4.0,6.0,8.0,10 \mathrm{~atm}, 200-2000 \mathrm{~K}$ 


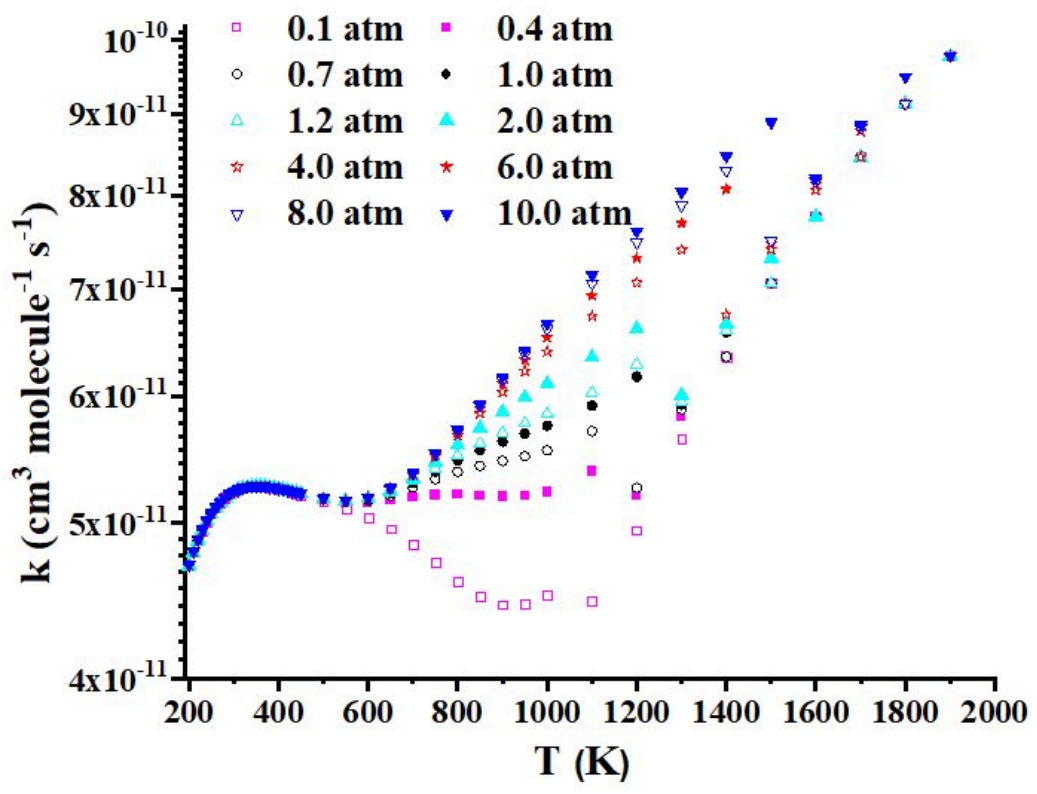

2276

2277 Figure S2.2. Final total rate coefficients $\left(\mathrm{cm}^{3}\right.$ molecule $\left.{ }^{-1} \mathrm{~s}^{-1}\right)$ of 2-MP $+\mathrm{OH}$ at 0.1, 0.4, 0.7, 1.0, $2278 \quad 1.2,2.0,4.0,6.0,8.0,10 \mathrm{~atm}, 200-2000 \mathrm{~K}$

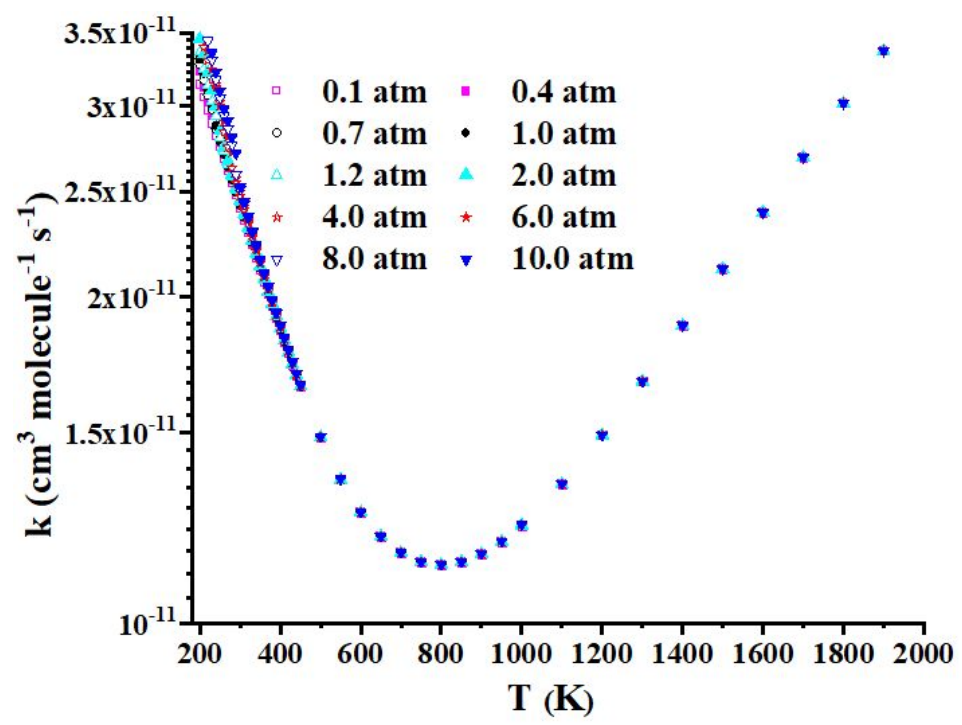

10) Figure S3.1. The rate coefficients of various reaction channels of $3-\mathrm{MH}+\mathrm{OH}$ at $0.1,0.4$, $0.7,1.0,1.2,2.0,4.0,6.0,8.0,10 \mathrm{~atm}, 200-200 \mathrm{~K}$ 

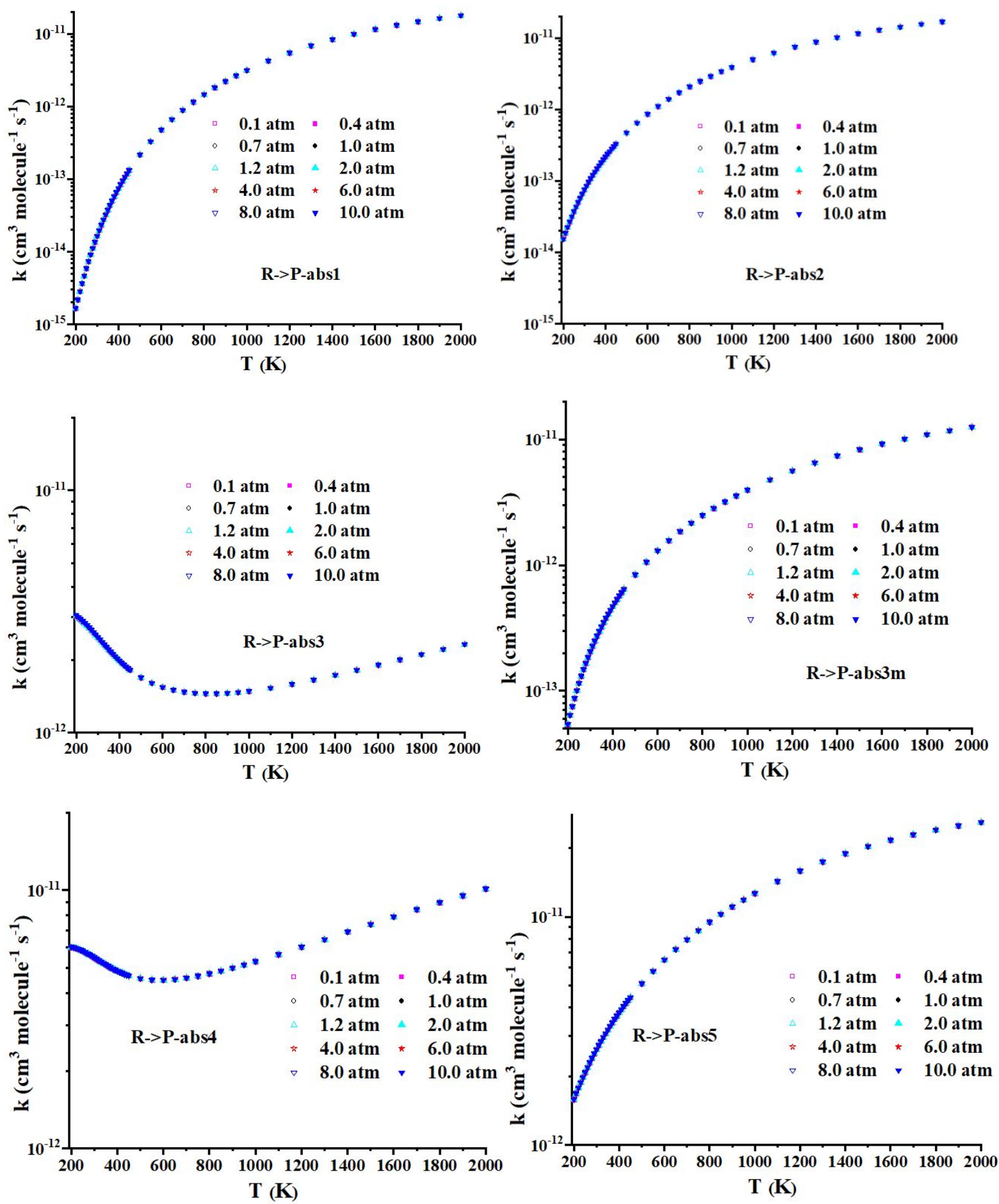

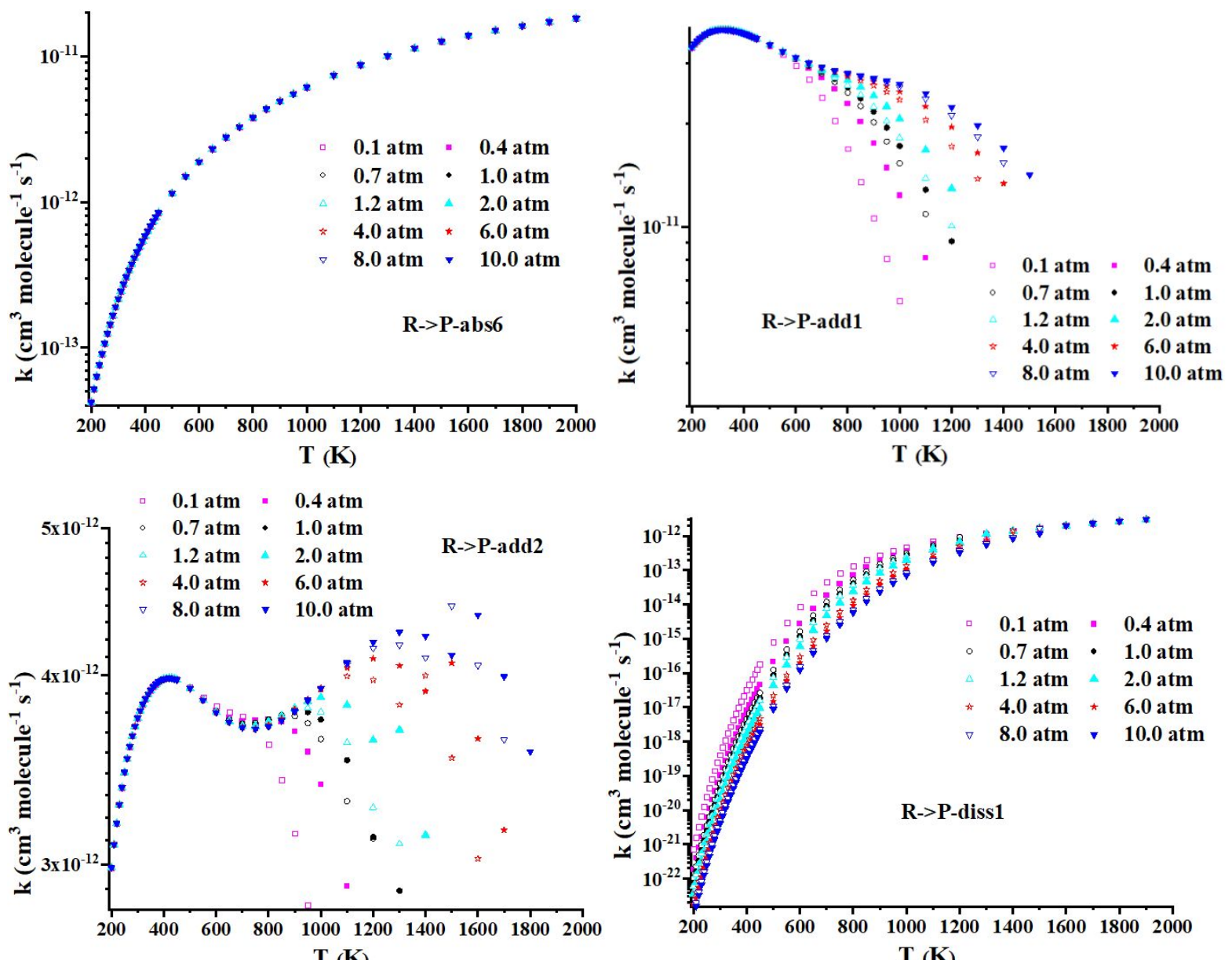

$\mathbf{T}(\mathbf{K})$

$\mathbf{T}(\mathbf{K})$

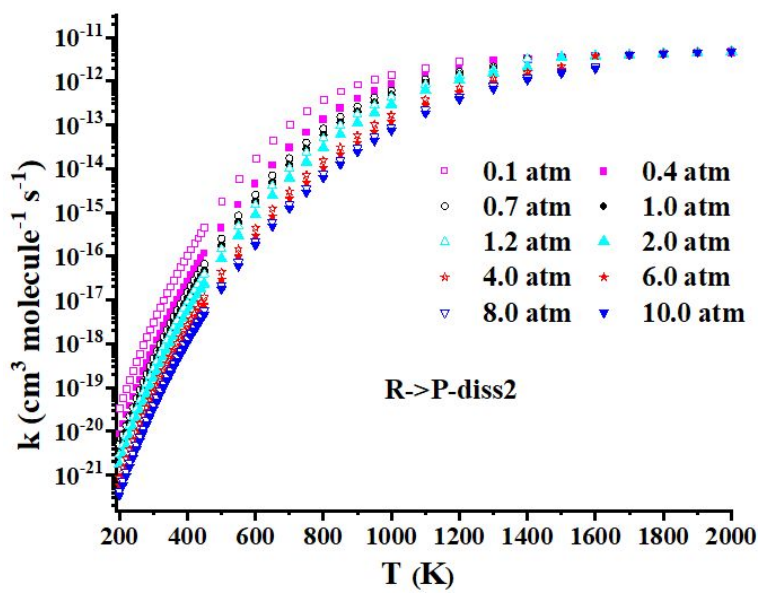

Figure S3.2. The rate coefficients of various reaction channels of 2-MP $+\mathrm{OH}$ at $0.1,0.4$, 

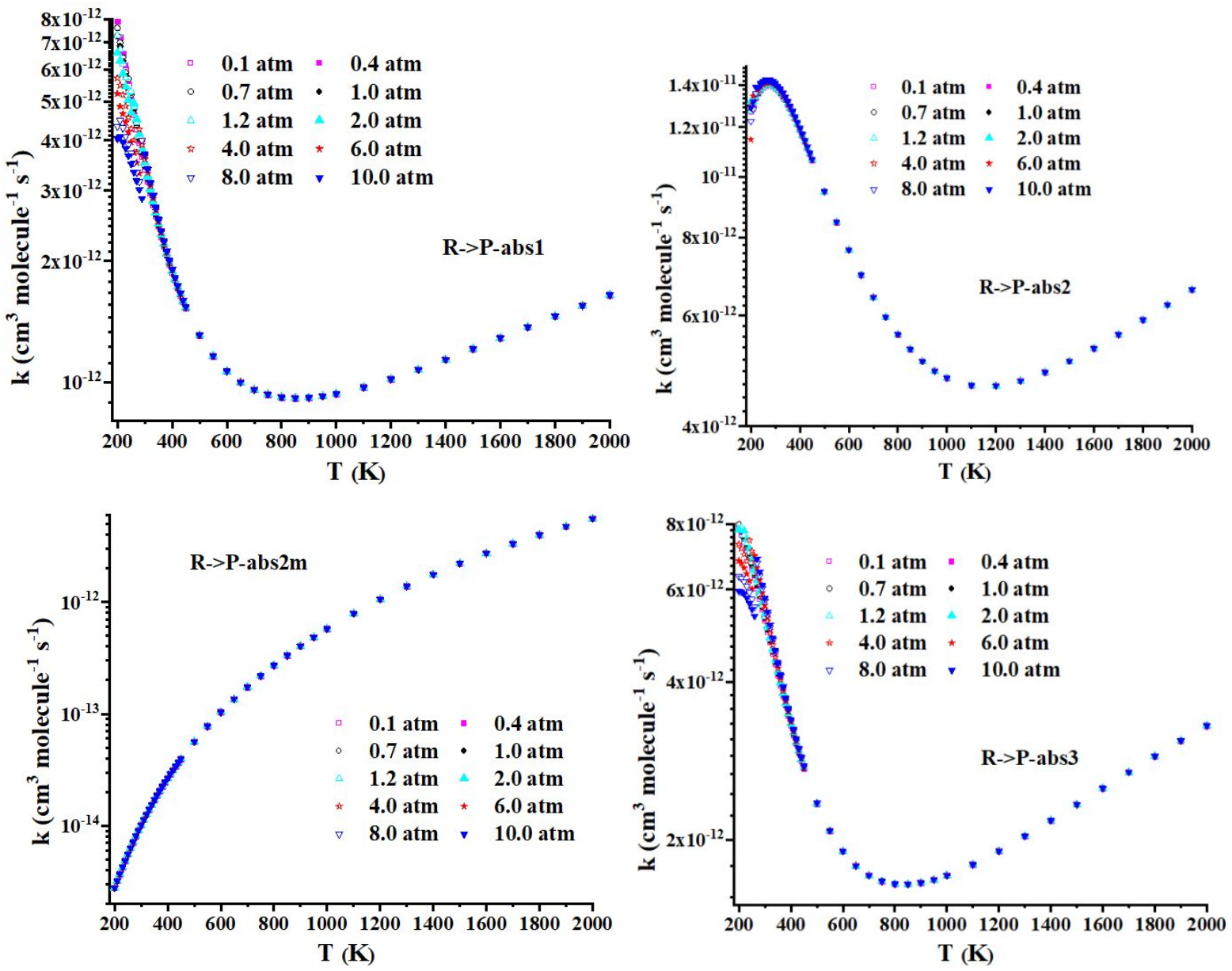

2295
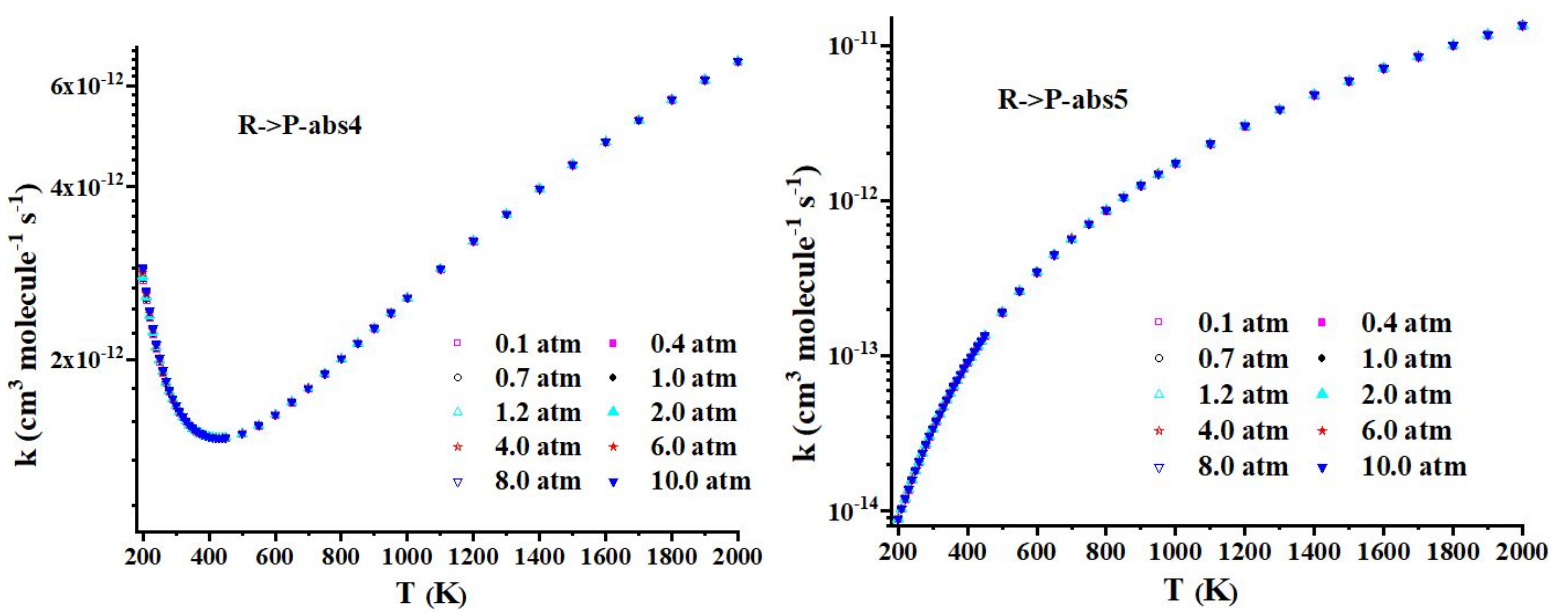

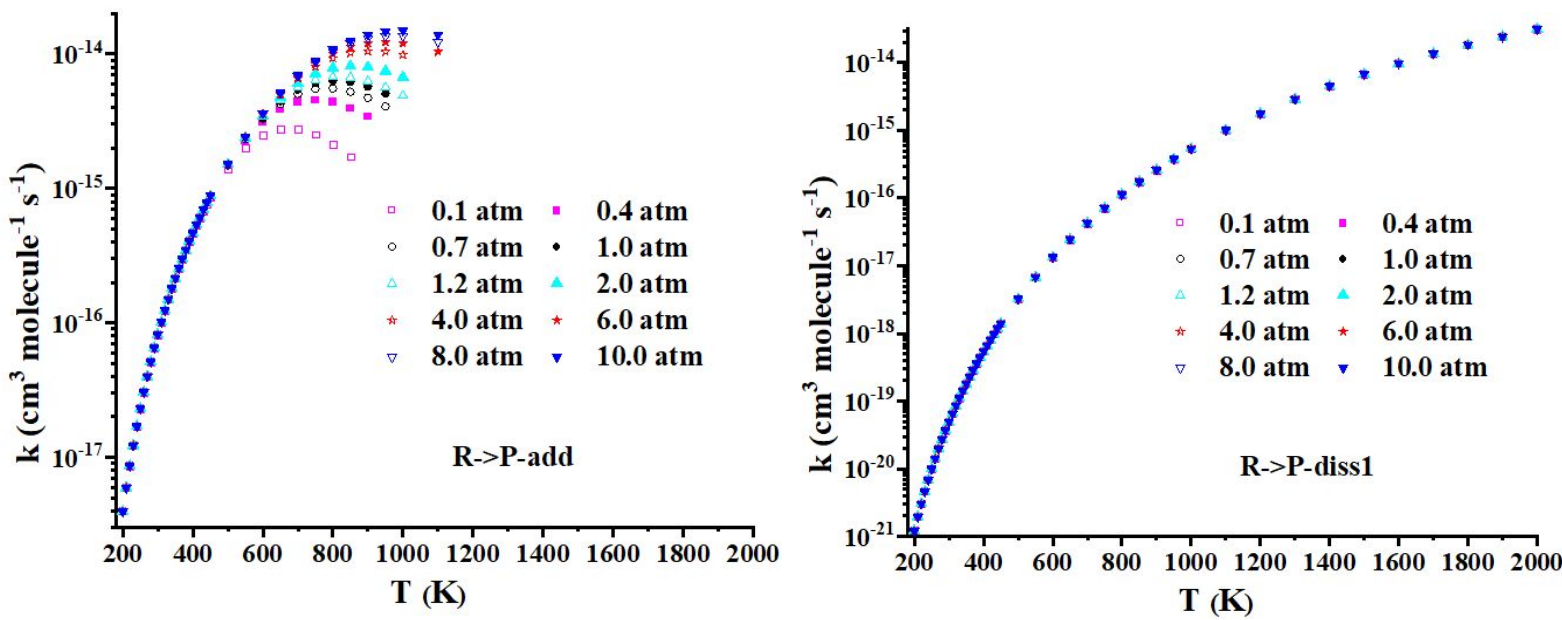

2299
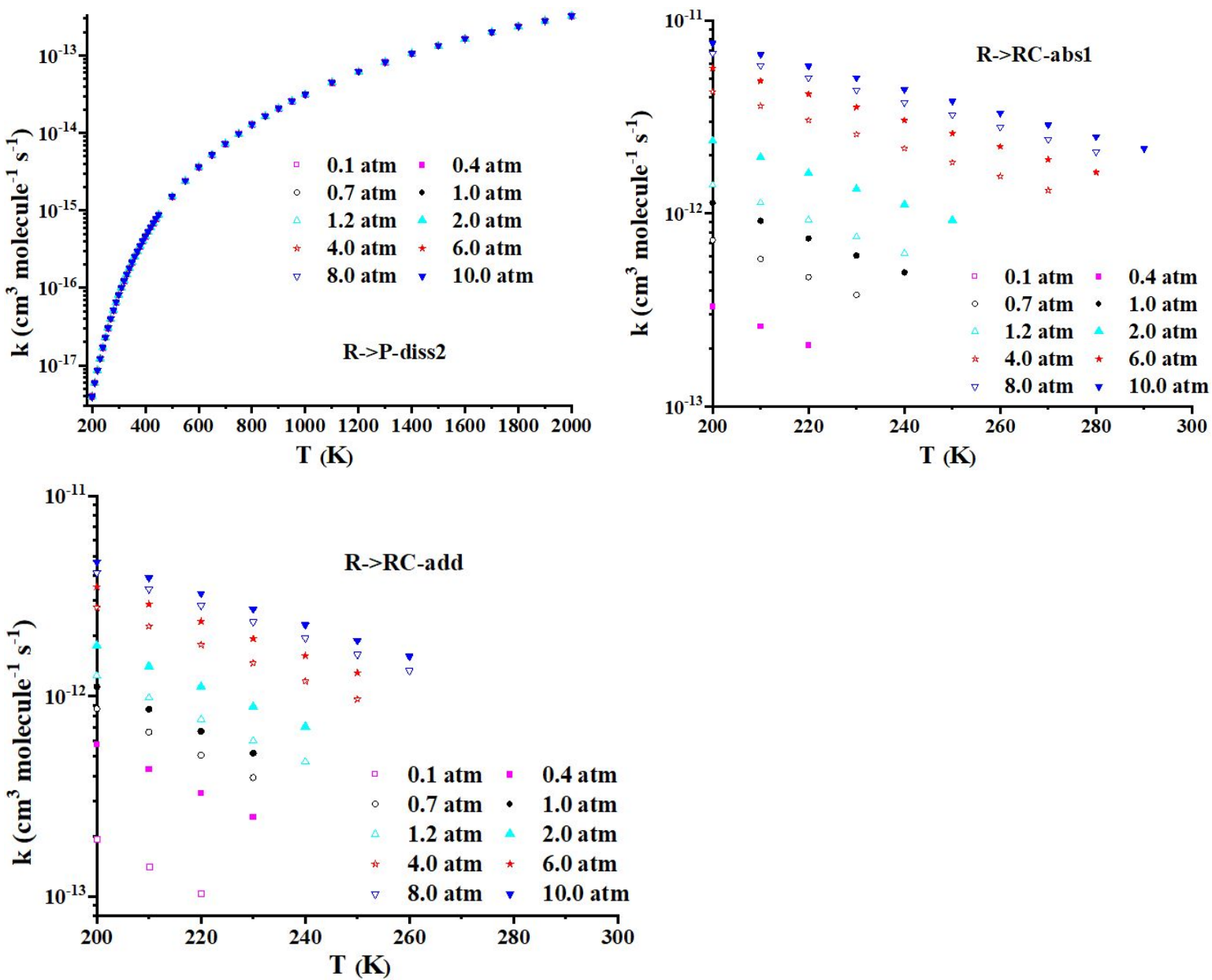

11) Figure S4.1 The branching ratios of $3-\mathrm{MH}+\mathrm{OH}$ for various reaction channels at $0.1,0.4$, $0.7,1.0,1.2,2.0,4.0,6.0,8.0,10 \mathrm{~atm}, 200-200 \mathrm{~K}$ 


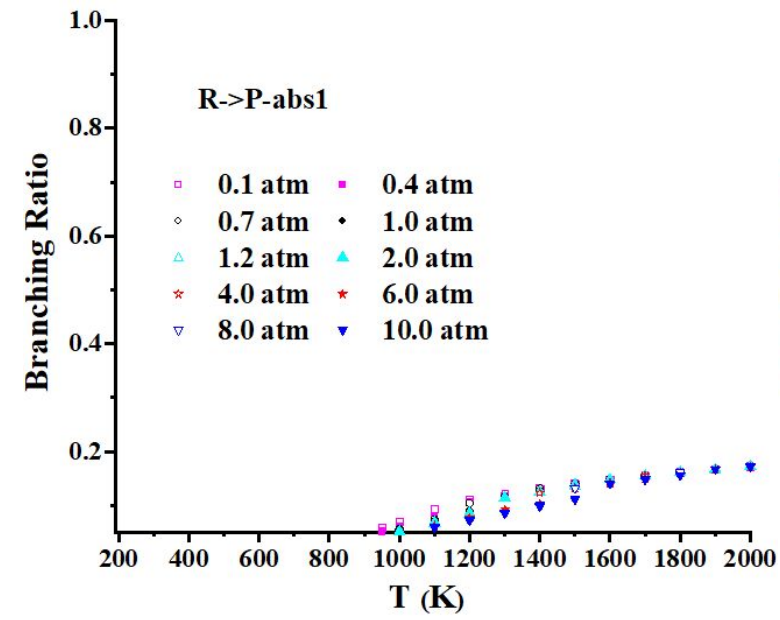

2306

2307
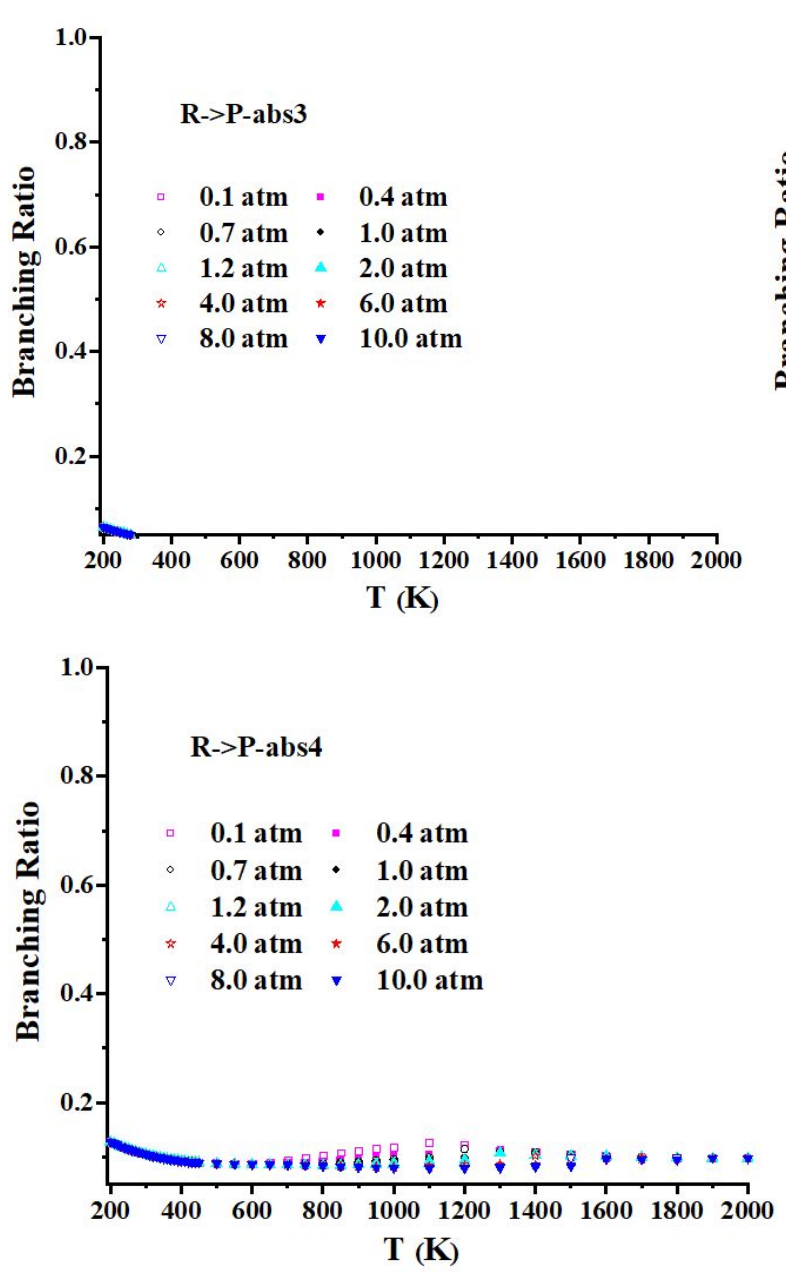
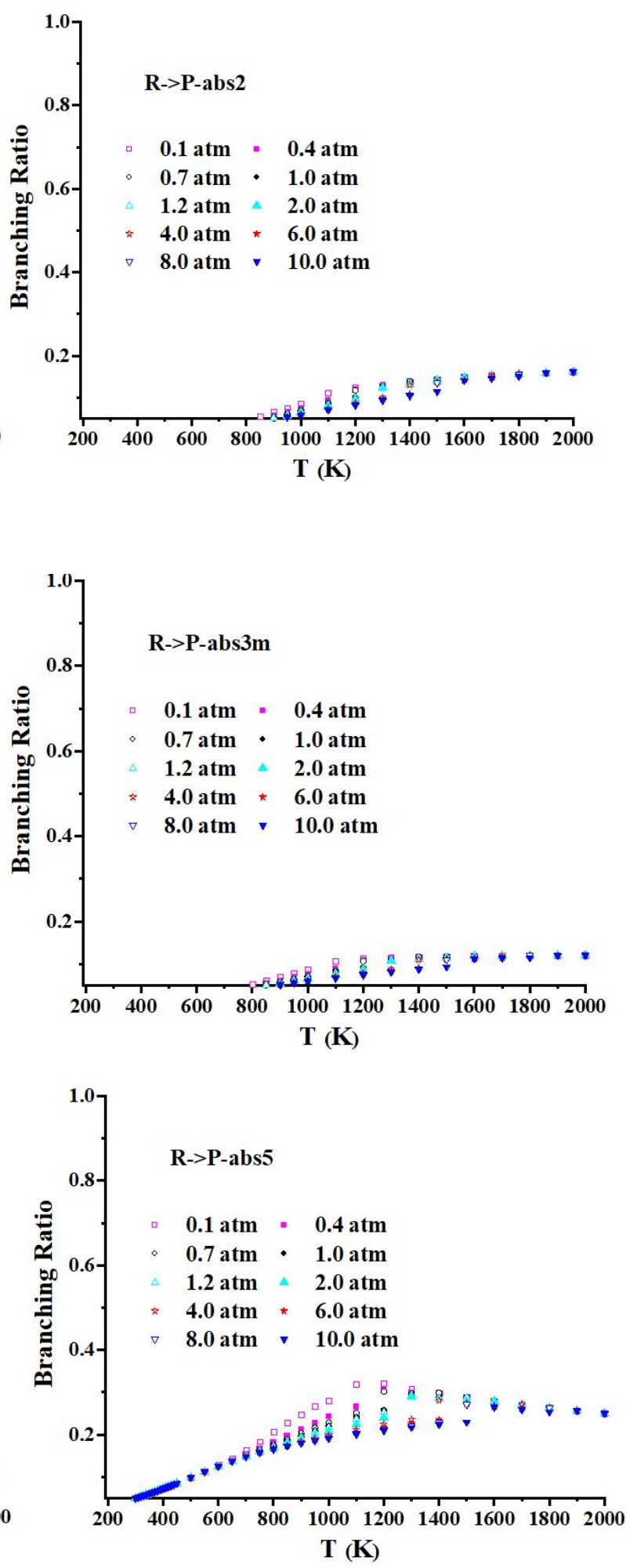


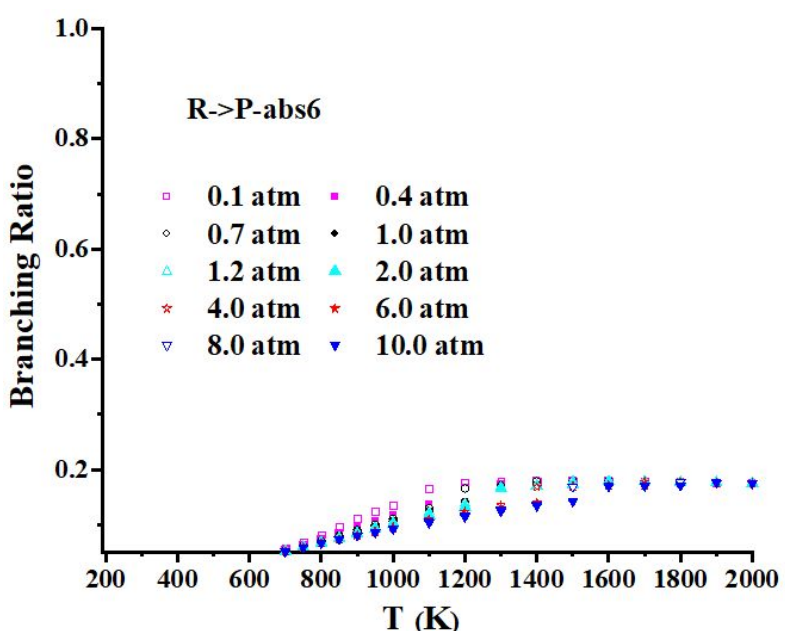

2309

2310

2311

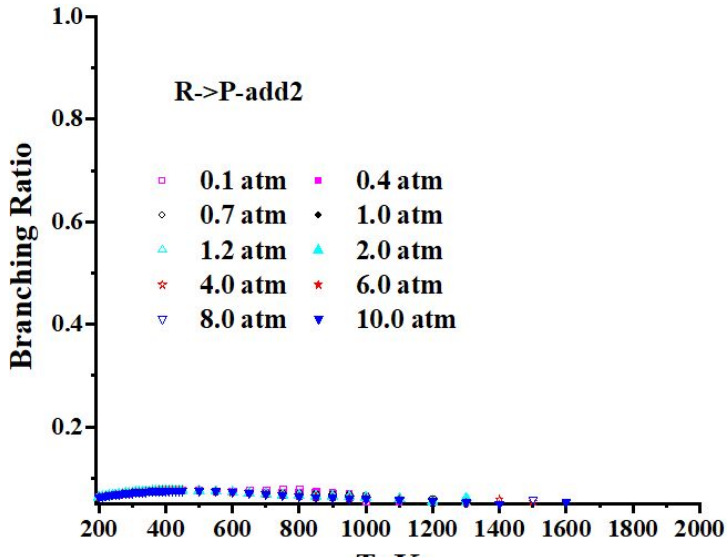
$\mathbf{T}(\mathbf{K})$

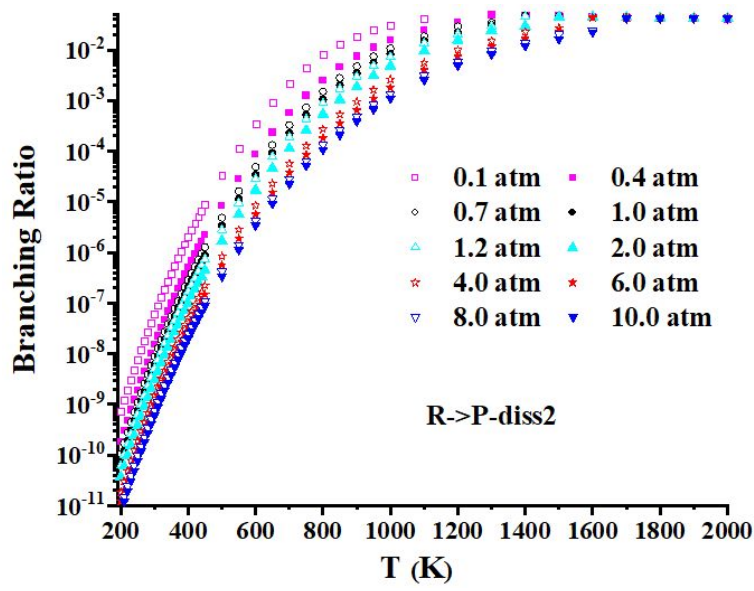

2312

2313 Figure S4.2 The branching ratios of $2-\mathrm{MP}+\mathrm{OH}$ for various reaction channels at $0.1,0.4,0.7$,
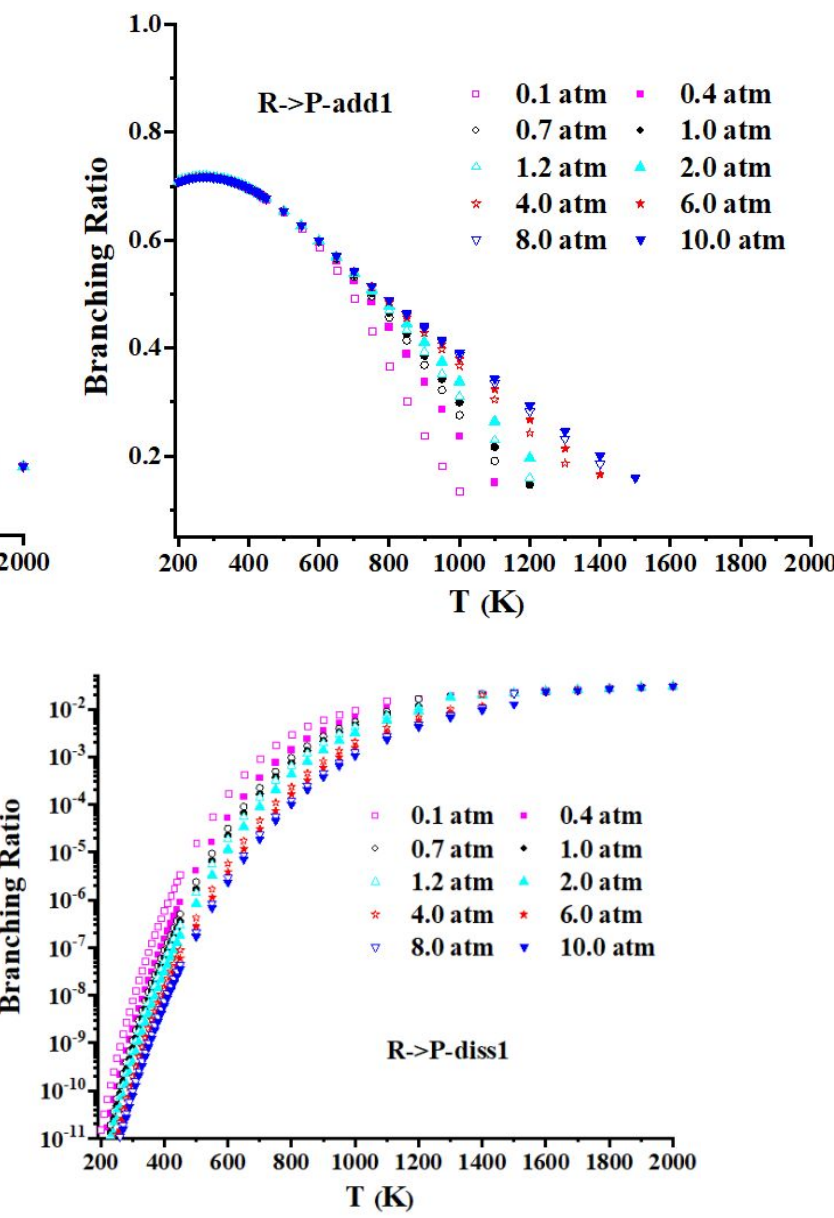

$\mathbf{T}(\mathbf{K})$ 

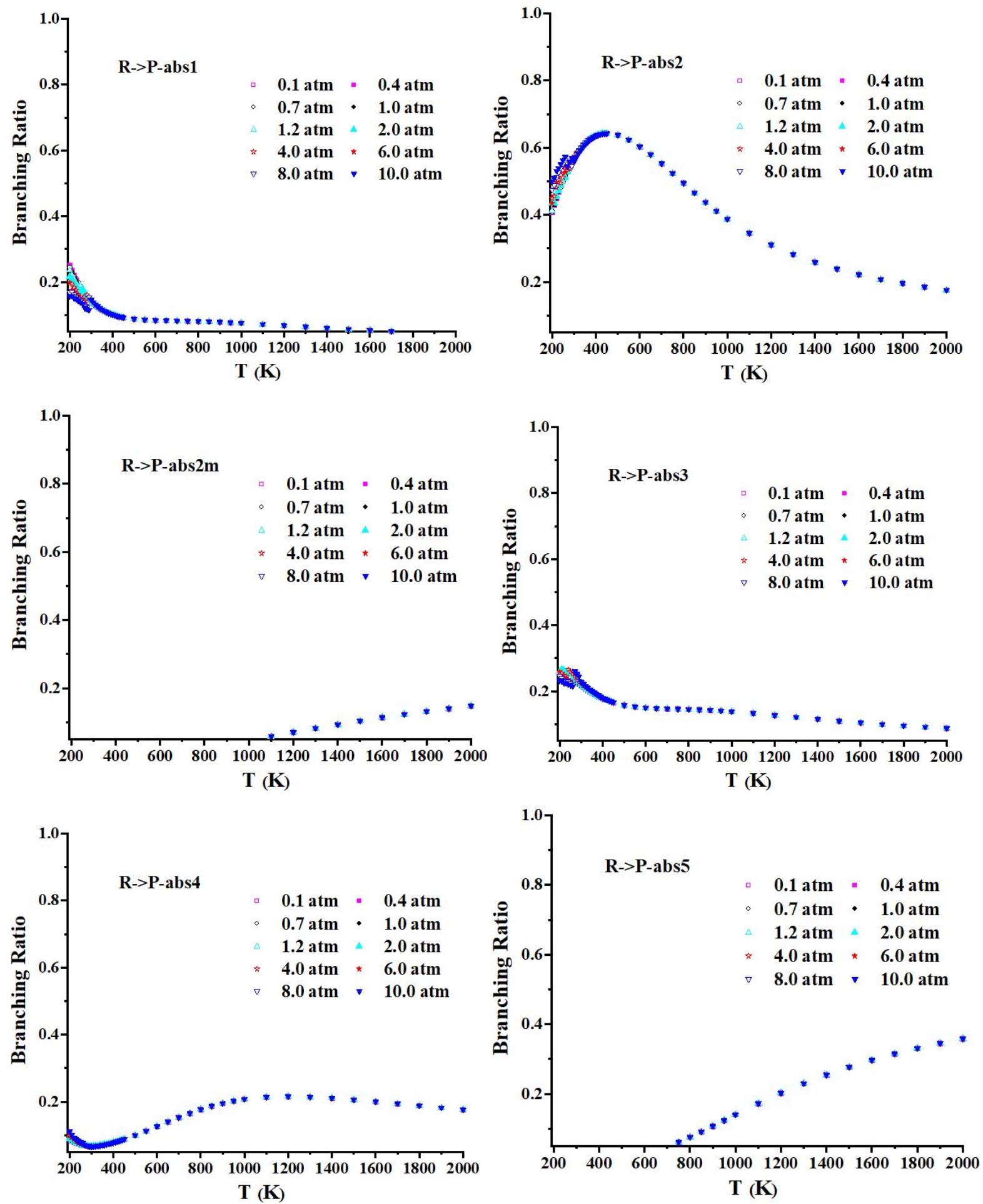

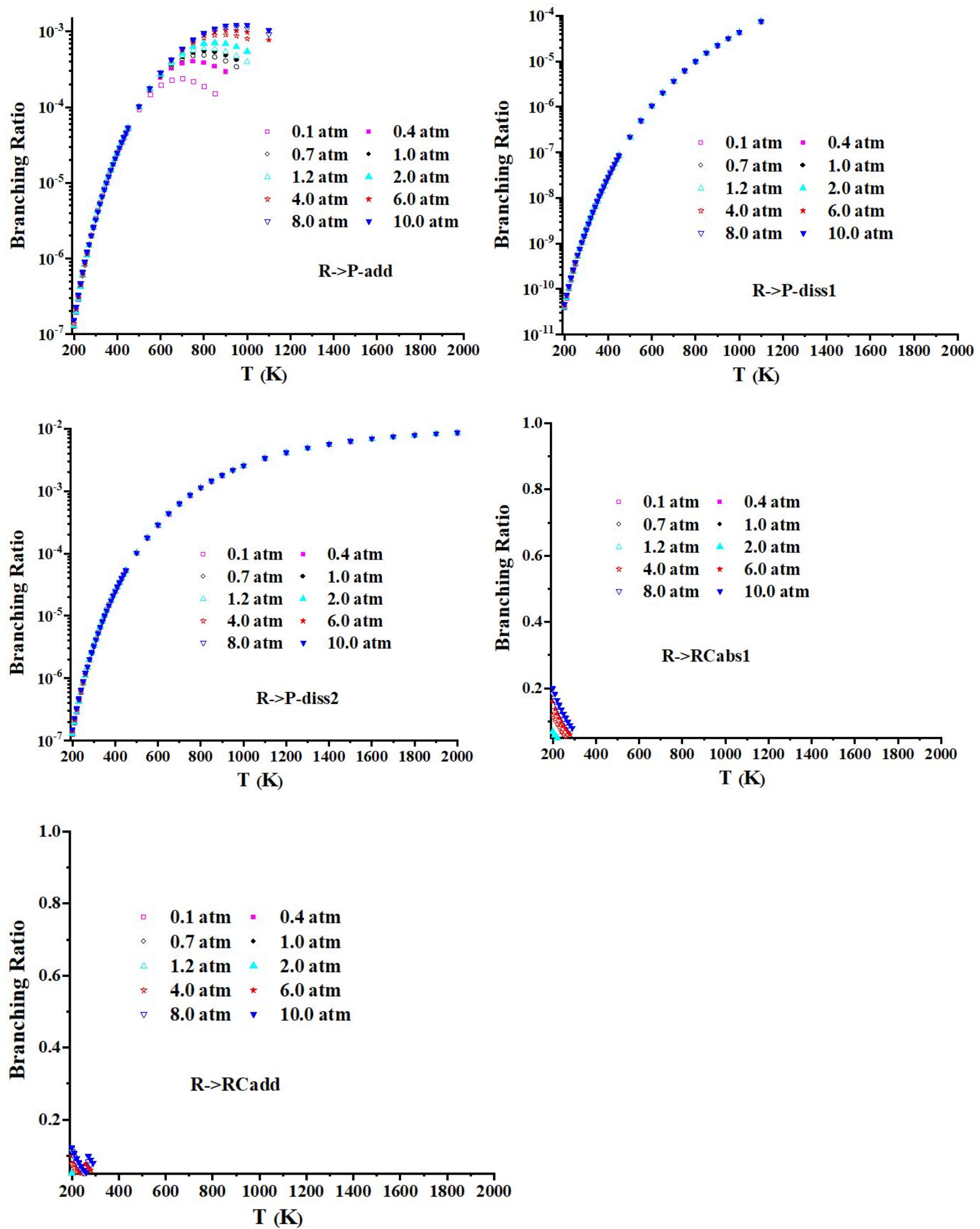

12) Figure S5.1. The rate coefficients of $\mathrm{OH}$ addition, $\mathrm{H}$ abstraction and addition-dissociation reactions for $3-\mathrm{MH}+\mathrm{OH}$ at $0.1,0.4,0.7,1.0,1.2,2.0,4.0,6.0,8.0,10 \mathrm{~atm}, 200-200 \mathrm{~K}$ 

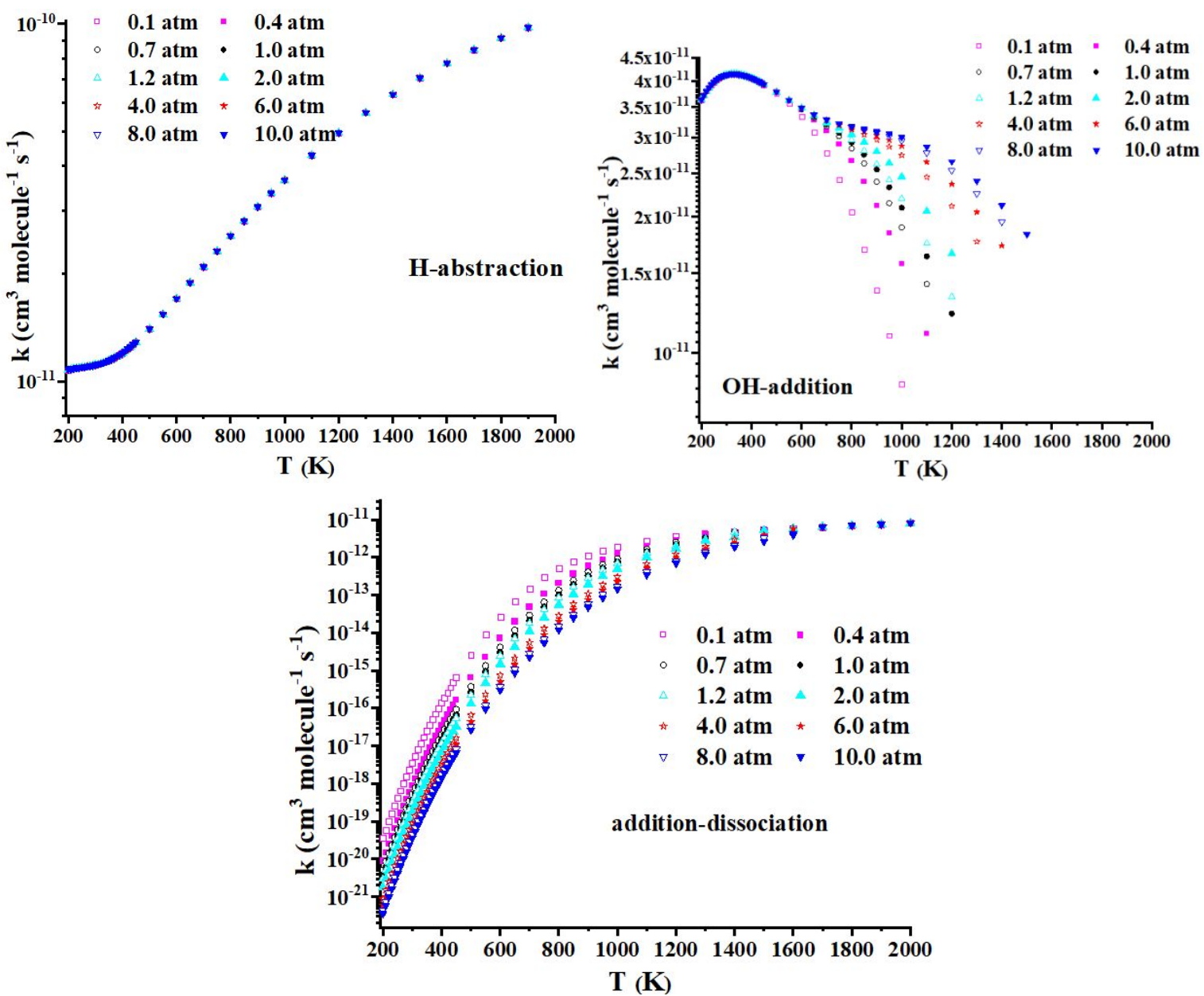

Figure S5.2. The rate coefficients of $\mathrm{OH}$ addition, $\mathrm{H}$ abstraction and addition-dissociation reactions for $2-\mathrm{MP}+\mathrm{OH}$ at $0.1,0.4,0.7,1.0,1.2,2.0,4.0,6.0,8.0,10 \mathrm{~atm}, 200-200 \mathrm{~K}$

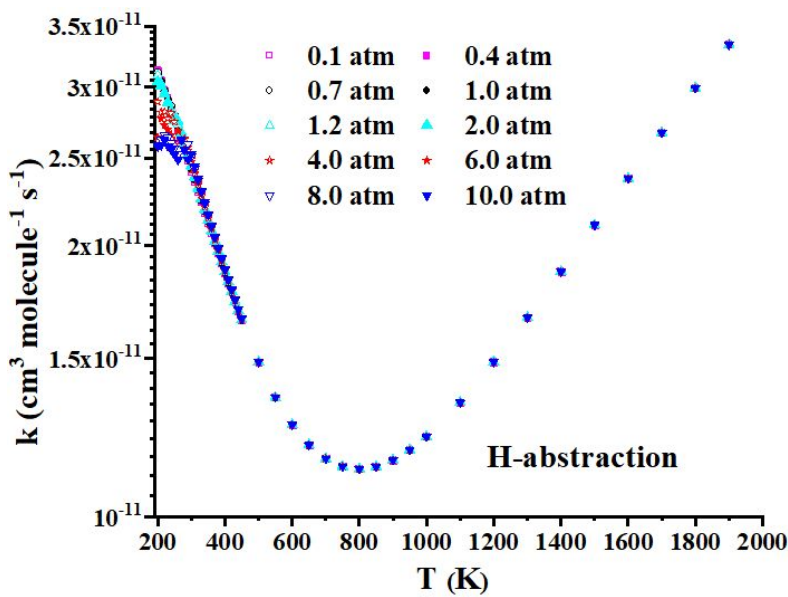

$\mathbf{T}(\mathbf{K})$

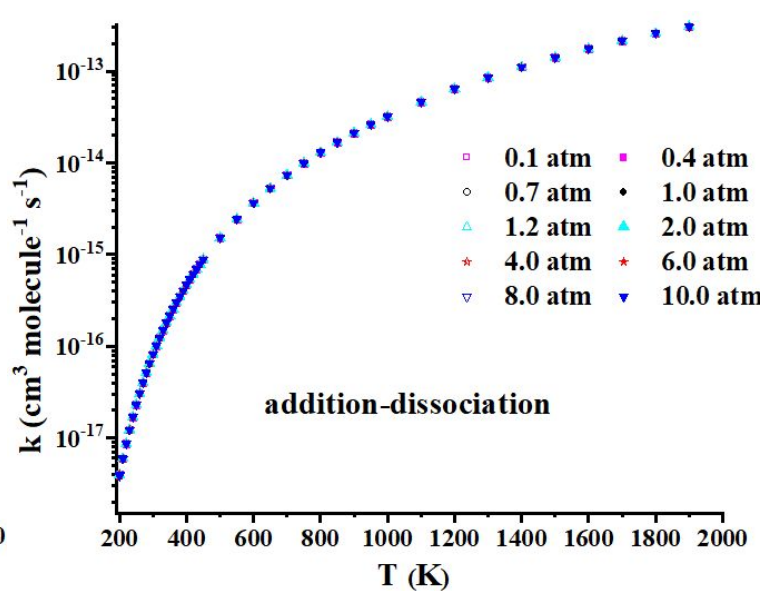

$\mathbf{T}(\mathbf{K})$ 


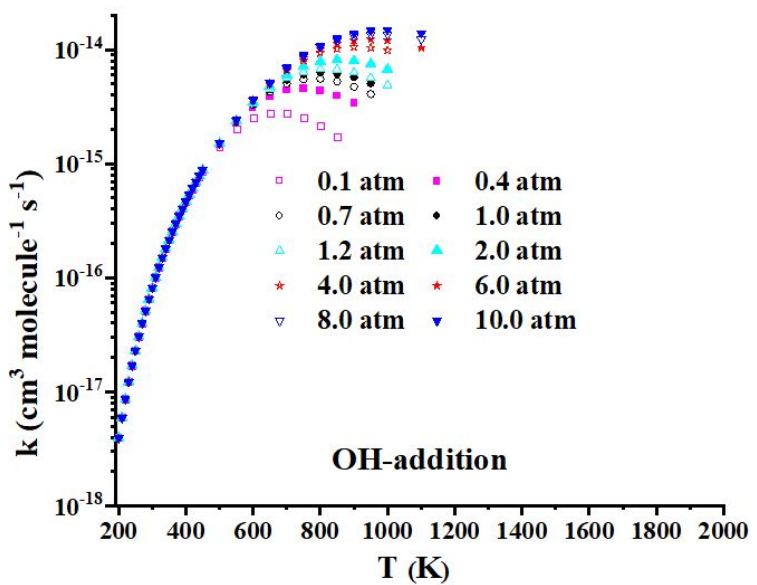

13) Figure S6.1 The branching ratios of $3-\mathrm{MH}+\mathrm{OH}$ addition, $\mathrm{H}$ abstraction and additiondissociation reactions at $0.1,0.4,0.7,1.0,1.2,2.0,4.0,6.0,8.0,10 \mathrm{~atm}, 200-200 \mathrm{~K}$
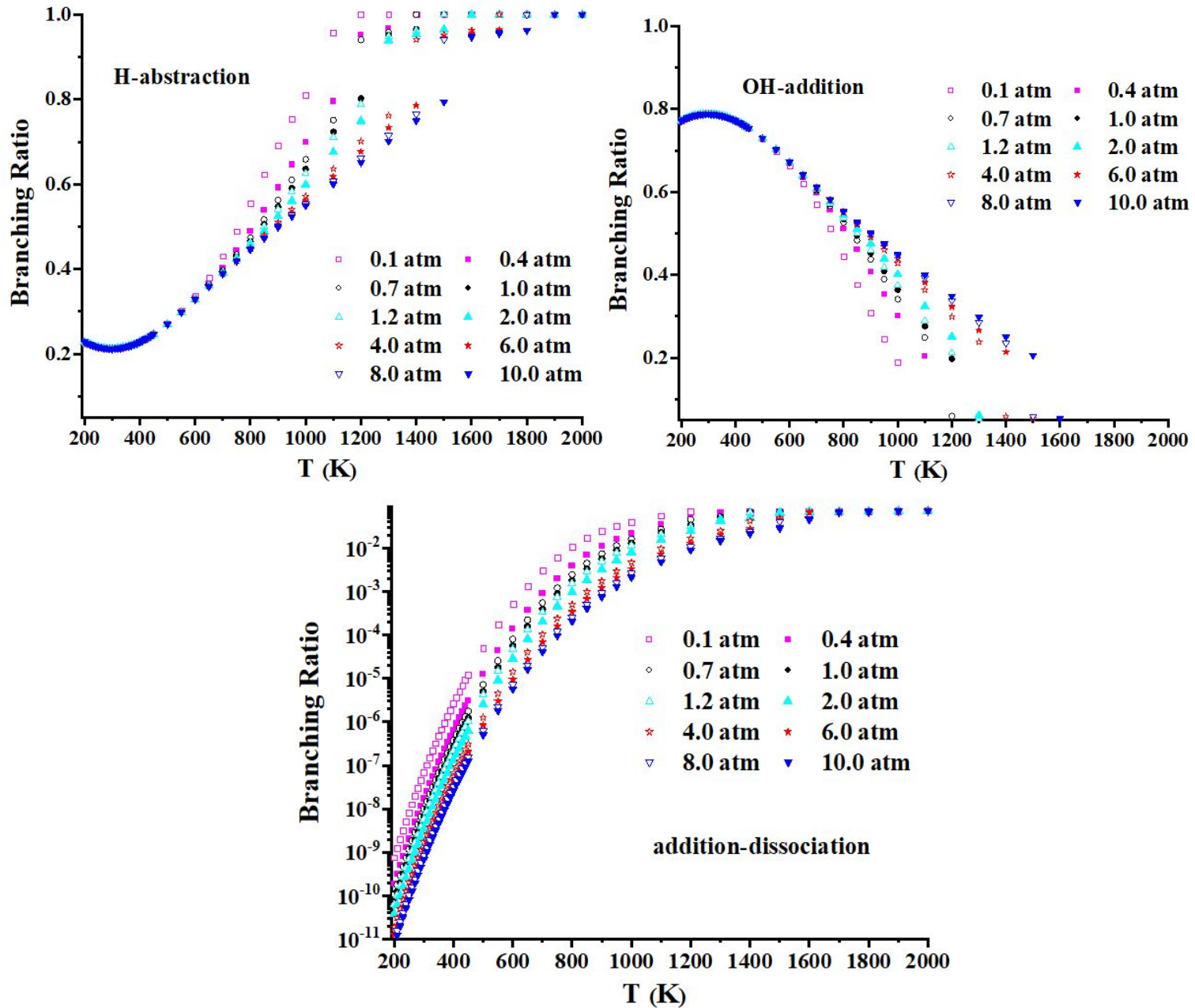

Figure S6.2 The branching ratios of $2-\mathrm{MP}+\mathrm{OH}$ addition, $\mathrm{H}$ abstraction and additiondissociation reactions at $0.1,0.4,0.7,1.0,1.2,2.0,4.0,6.0,8.0,10$ atm, 200-200K 


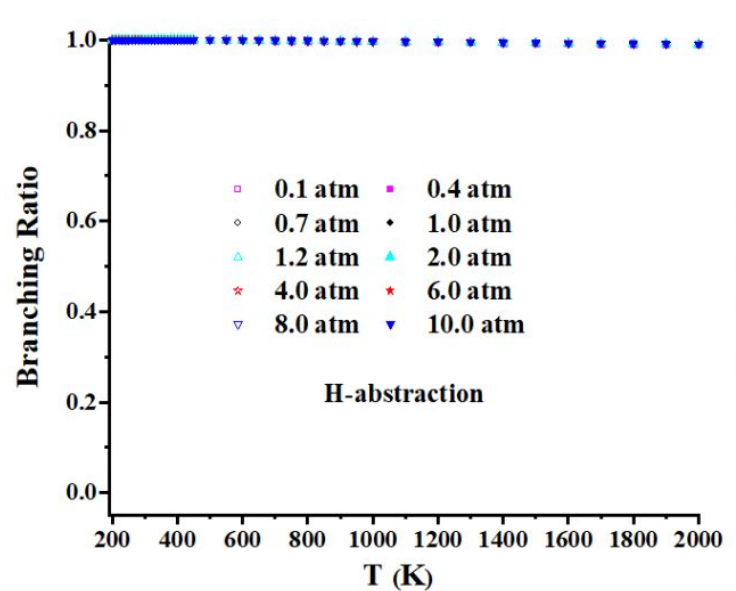

T (K)
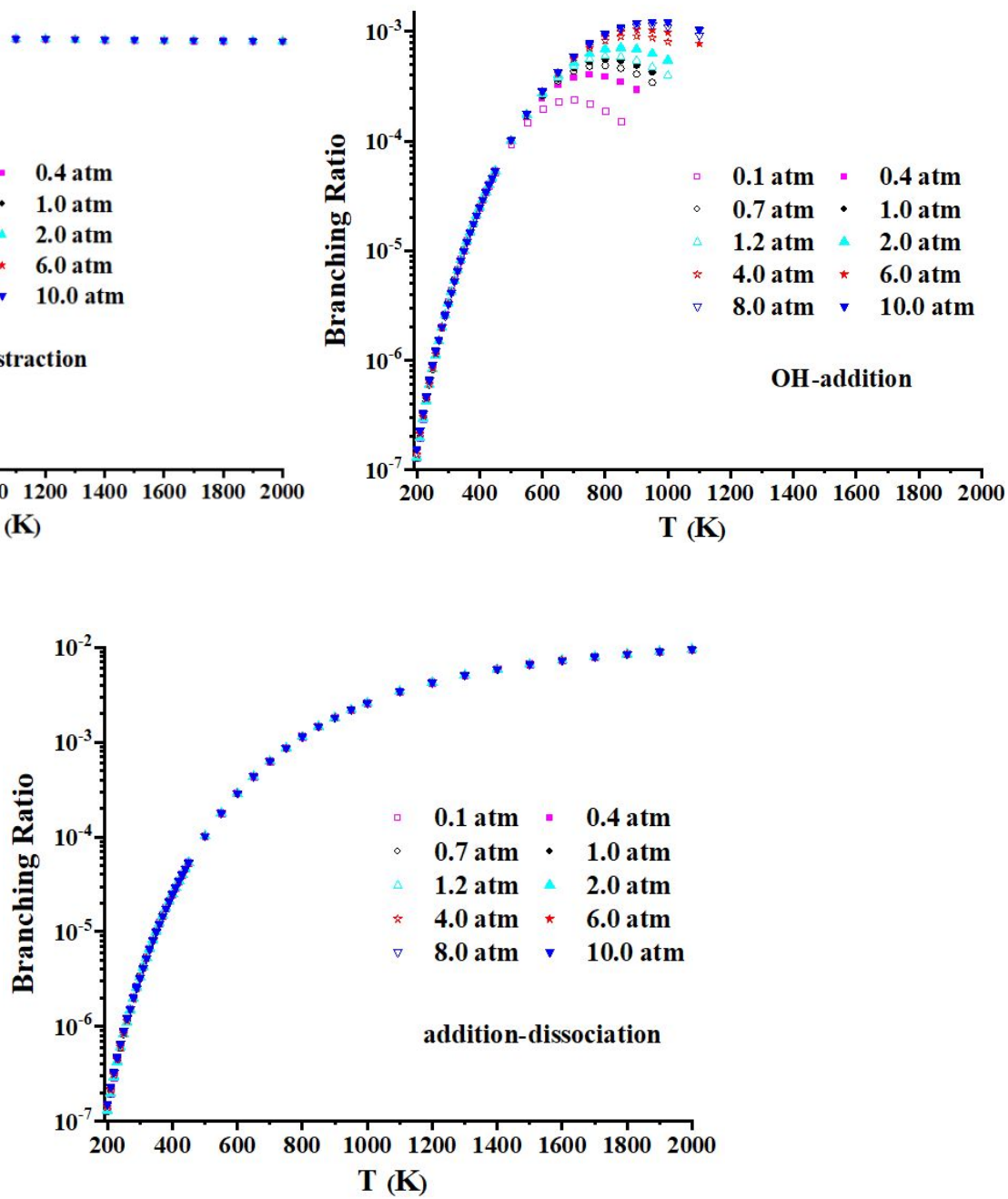

2342

14) Figure S7. The ratio of the rate coefficients $(R \rightarrow$ abs 1$)$ at 0.1 atm to that at 10 atm with the shift of RCabs1 energy

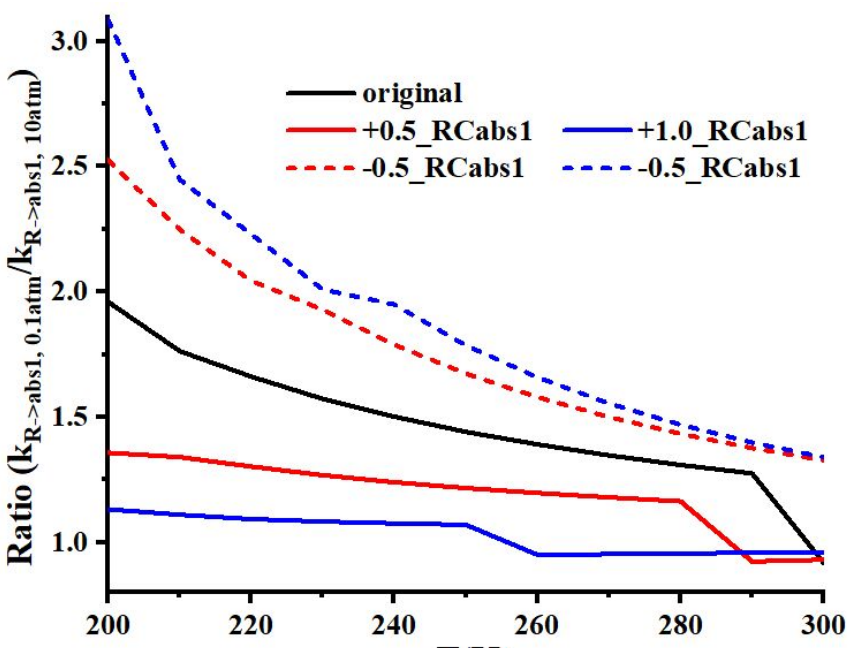

$\mathbf{T}(\mathbf{K})$

2347 A slight negative pressure dependence for rate coefficients of $\mathrm{R} \rightarrow$ abs1 (a well-skipping 
coefficients of $\mathrm{R} \rightarrow \mathrm{RCabs} 1$ channel present a positive pressure dependent effect. In Figure $\mathrm{S} 7$, we present the ratios of the rate coefficient $(\mathrm{R} \rightarrow \mathrm{abs} 1)$ at $0.1 \mathrm{~atm}$ to that at 10 atm with the shift of RCabs1 energy. As the energy of RCabs1 increases, the probability of the reactants being stable in this complex decreases. The pressure dependence is negligible when the energy of RCabs 1 is shifted by $+1.0 \mathrm{kcal} / \mathrm{mol}$, with a maximum factor of 1.1 at $200 \mathrm{~K}$. On the contrary, when the energy of RCabs1 is reduced, the reactants are more stabilized in the complex, resulting in a more significant pressure dependence. As a side note, we also checked the impact of tunneling on the pressure dependence by including or excluding the Eckart tunneling treatment for $\mathrm{TS}_{\mathrm{abs} 1}$ in the RRKM/ME simulation over the range of 200-2000K, 0.1-10atm. It turned out that the tunneling effect hardly influence the pressure-dependence of $k_{R} \rightarrow a b s 1$.

15) Figure S8. Ratios of rate coefficients for $3-\mathrm{MH}+\mathrm{OH}$ addition channels with or without the pre-reaction complex.

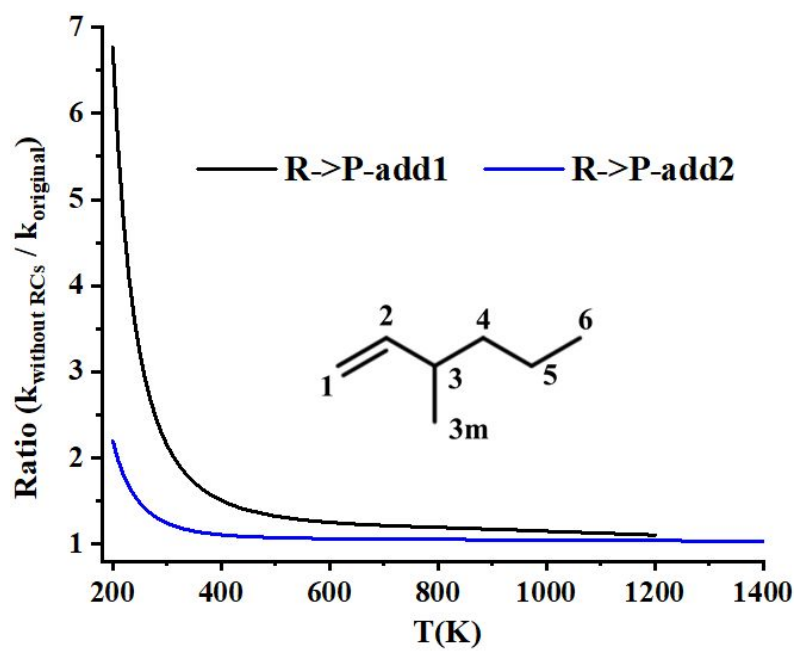

We further evaluate the role of van der Waals complexes in the reactions kinetics calculation. Figure S8 shows the ratios of rate coefficients for 1-add and 2-add by including or excluding the complex on $3-\mathrm{MH}+\mathrm{OH}$ PES. From 200-450K, the impact of complex gradually decreases with a maximum factor of 6.8 for 1 -add, 2.2 for 2 -add at $200 \mathrm{~K}$, and 2.2 for 1 -add, 1.2 for 2 add at $300 \mathrm{~K}$. In addition, increasing or decreasing the potential well depth of complexes by 0.2 to $1.0 \mathrm{kcal} / \mathrm{mol}$ does not influence the computed RRKM/ME rate coefficients, which is similar to our previous conclusions concerning toluene $+\mathrm{OH}^{60}$ and styrene $+\mathrm{OH}^{61}$.

16) Figure S9.1 The total rate coefficients of $3-\mathrm{MH}+\mathrm{OH}$ after the barrier energy shift of \pm 0.2 
$\mathrm{kcal} / \mathrm{mol}$.

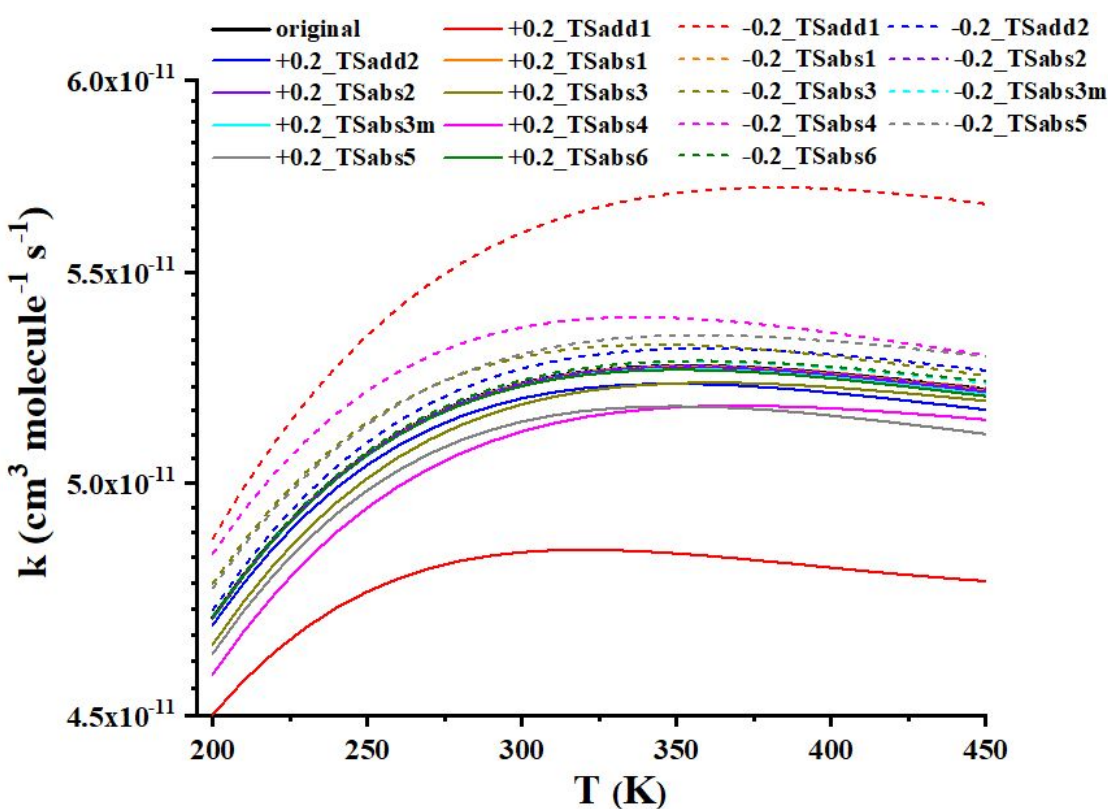

2375 Figure S9.2 The total rate coefficients of 2-MP $+\mathrm{OH}$ after the barrierenergy shift of \pm 0.2 $\mathrm{kcal} / \mathrm{mol}$.

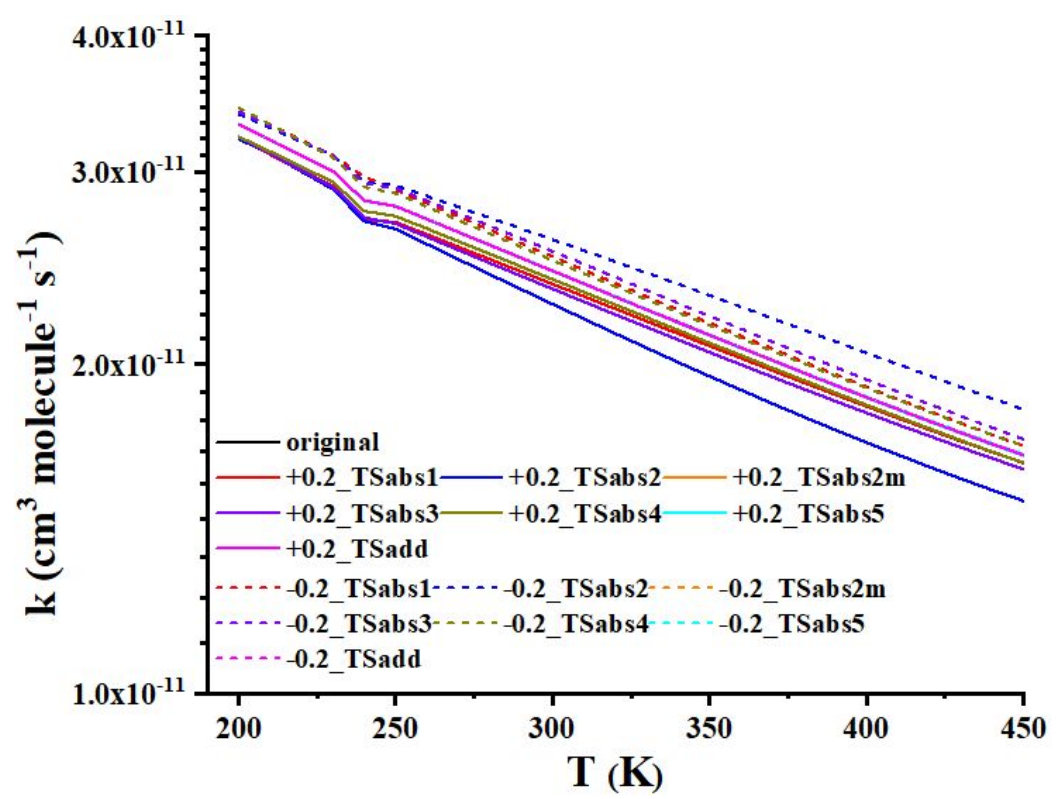

2379 In our previous study, ${ }^{60}$ we have evaluated the uncertainty sources of the calculated rate 2380 coefficients and branching ratios for toluene $+\mathrm{OH}$. The results indicated that the energy parameters contribute most to those of accuracy under atmospheric conditions. ${ }^{60}$ In order to estimate the accuracy of the results presented in this work, we have shifted the energy of various transition states by $\pm 0.2 \mathrm{kcal} / \mathrm{mol}$ considering the theoretical level we used. Figure 
S9 presents the effects of the energy barriers on total rate coefficients over the temperature range of $200-450 \mathrm{~K}$, and $1 \mathrm{~atm}$. Taking $3-\mathrm{MH}+\mathrm{OH}$ as an example, the total rate coefficient computed at $300 \mathrm{~K}$ is $5.2 \times 10^{-11} \mathrm{~cm}^{3}$ molecule $\mathrm{e}^{-1} \mathrm{~s}^{-1}$. Reducing the energy of $\mathrm{TS}_{\mathrm{add} 1}$ significantly 2387 increases the rate coefficients at low temperature $\left(5.6 \times 10^{-11} \mathrm{~cm}^{3}\right.$ molecule $\mathrm{s}^{-1}$ at $\left.300 \mathrm{~K}\right)$. 2388 Instead, enhancing its energy decreases the rate coefficient $\left(4.9 \times 10^{-11} \mathrm{~cm}^{3}\right.$ molecule $\mathrm{s}^{-1}$ at $300 \mathrm{~K}$ ). Changing the energy of the critical species can result in $68 \%$ (increasing the energy of $2390 \mathrm{TS}_{\mathrm{add} 1}$ ) to $75 \%$ (reducing the energy of $\mathrm{TS}_{\mathrm{add} 1}$ ) for the branching ratios of the dominant product 2391 (add1). The detailed variations for the branching ratios of 3-MH $+\mathrm{OH}$ after the barrier shift of $2392 \pm 0.2 \mathrm{kcal} / \mathrm{mol}$ are shown in Figure S10. Therefore, we set the sig figs on total rate coefficients and $\%$ number to be one and zero digits in this work.

17) Figure S10.1 The branching ratios of $3-\mathrm{MH}+\mathrm{OH}$ after the barrier shift of $\pm 0.2 \mathrm{kcal} / \mathrm{mol}$

2396
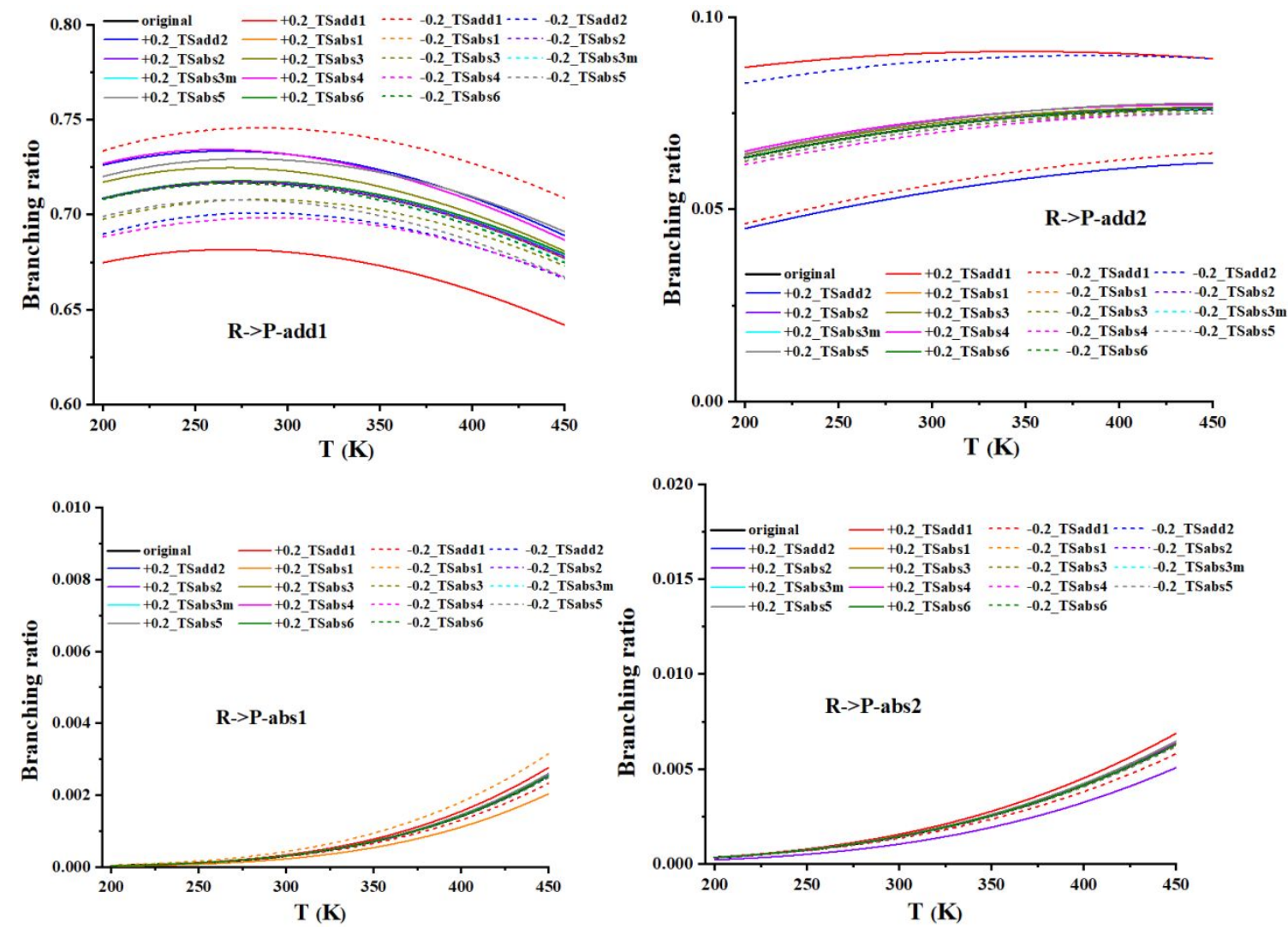

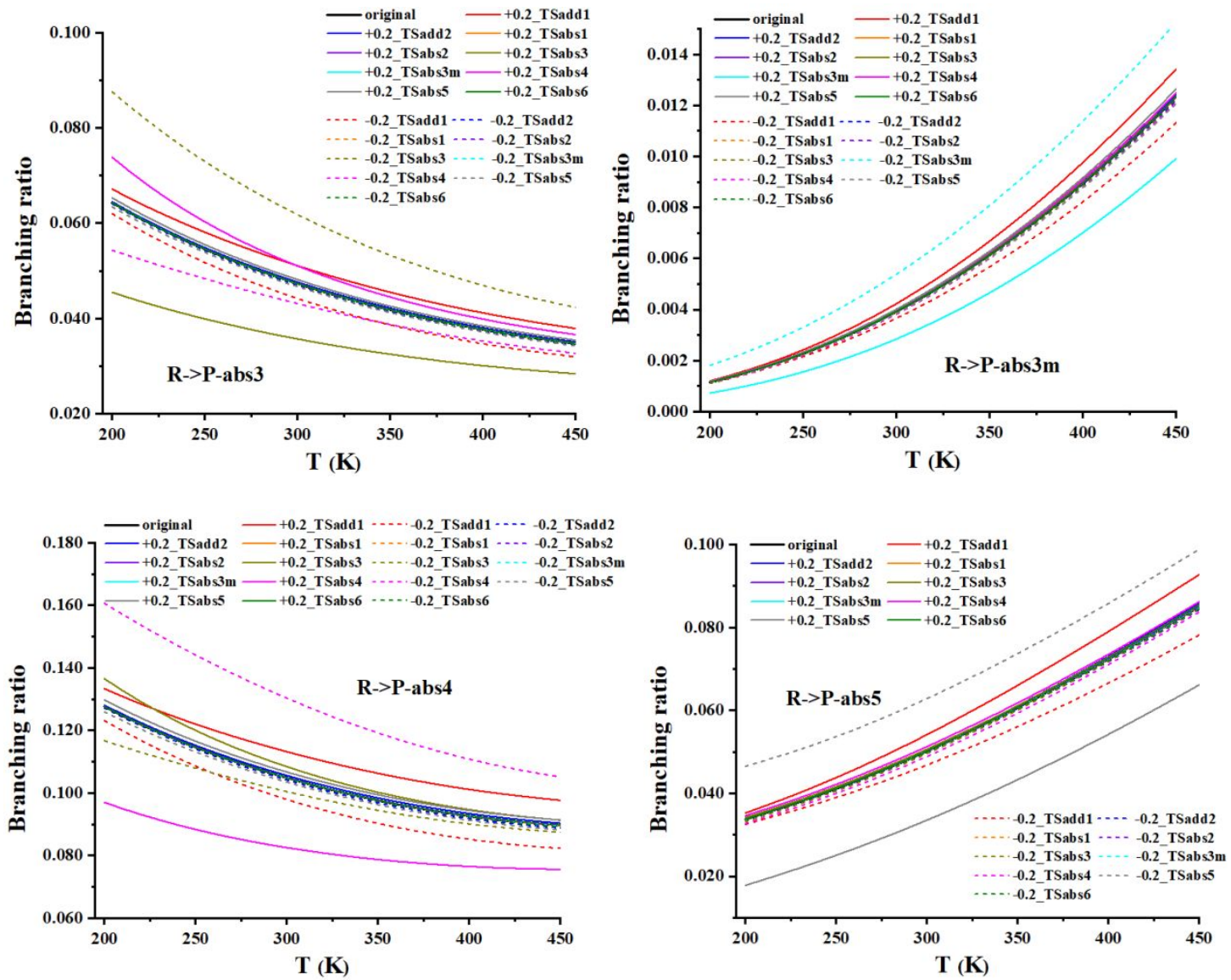

2400
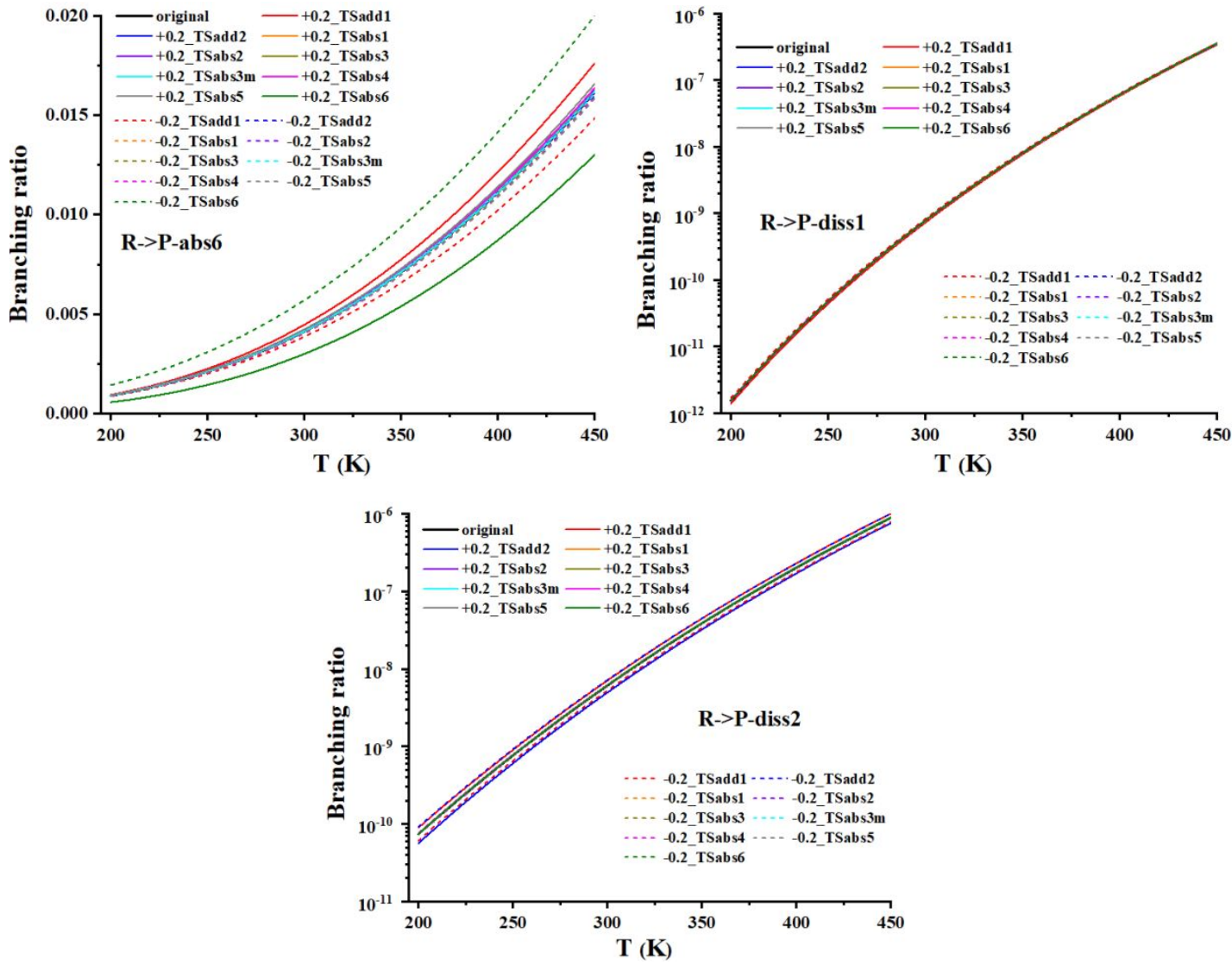
Figure S10.2 The branching ratios of 3-MH+ OH after the barrier shift of $\pm 0.2 \mathrm{kcal} / \mathrm{mol}$
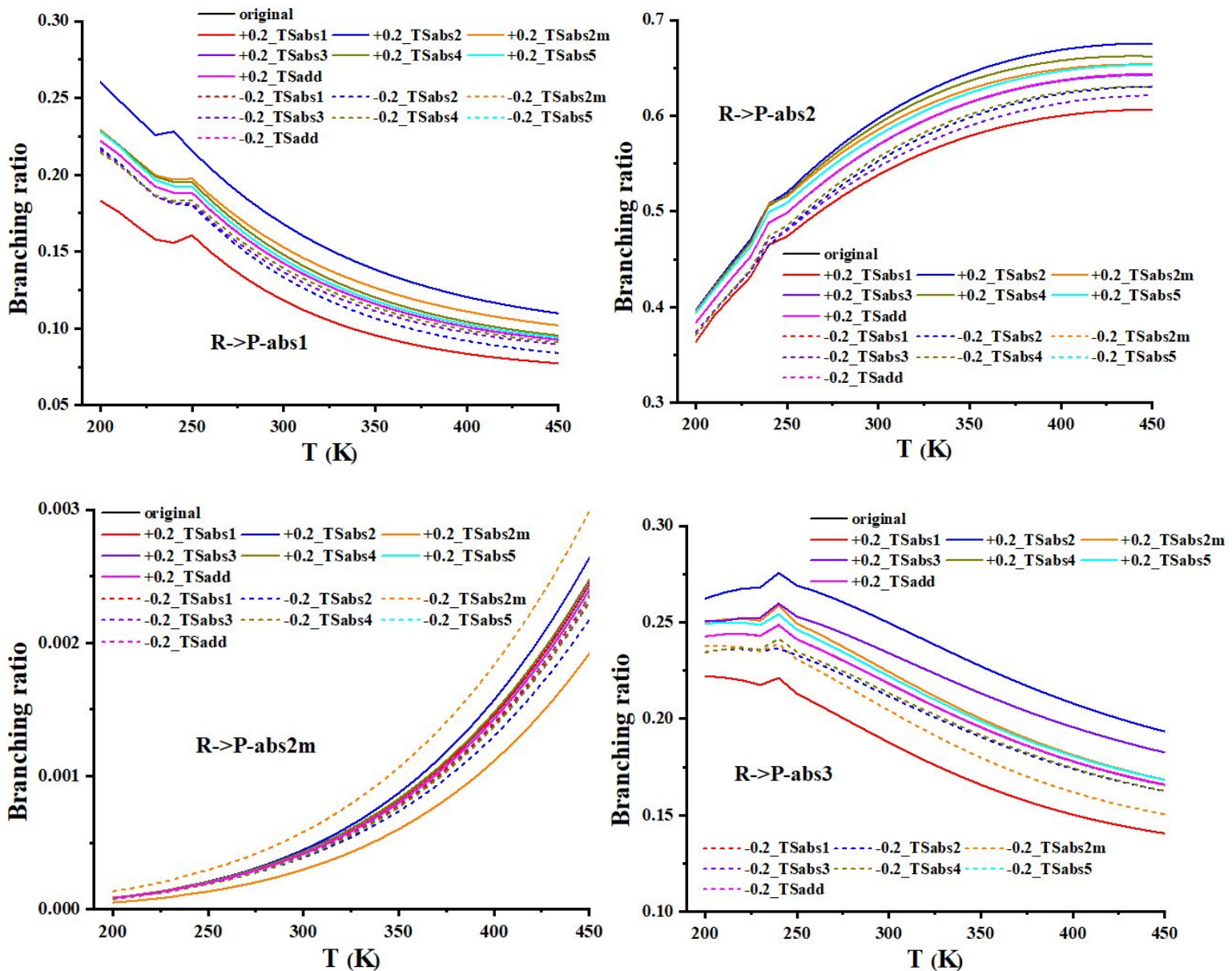

2406
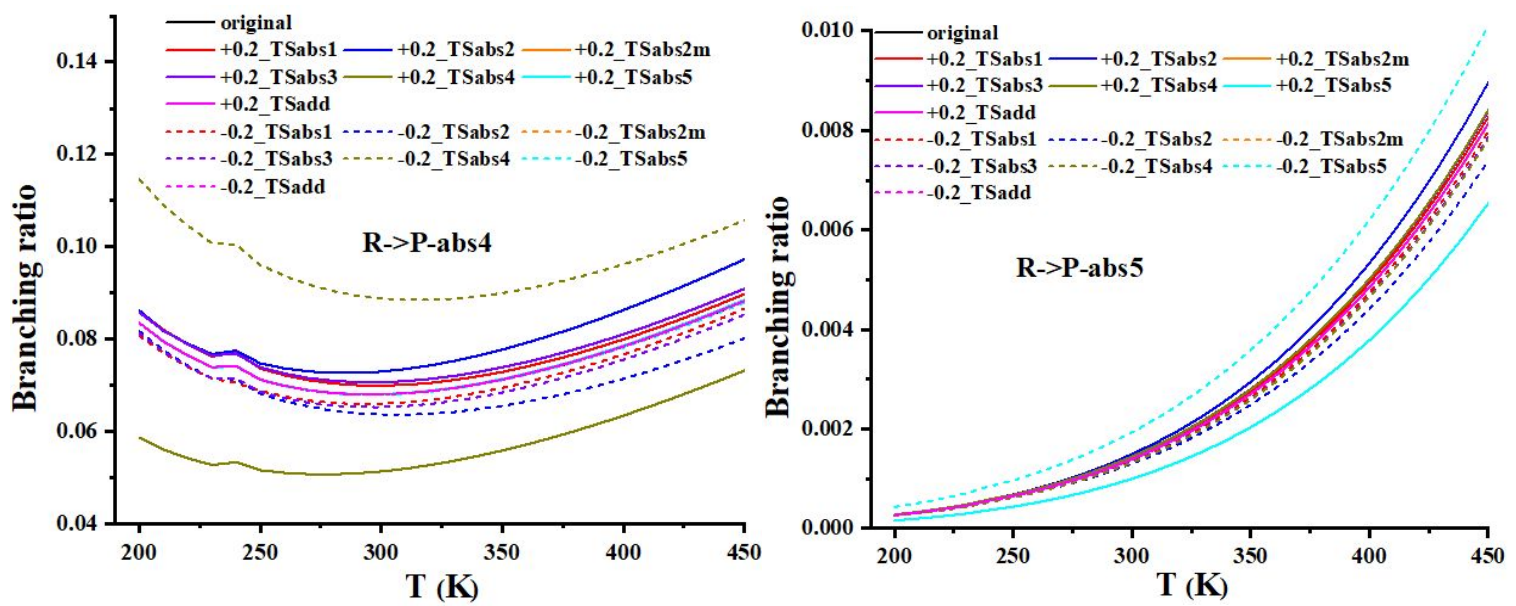

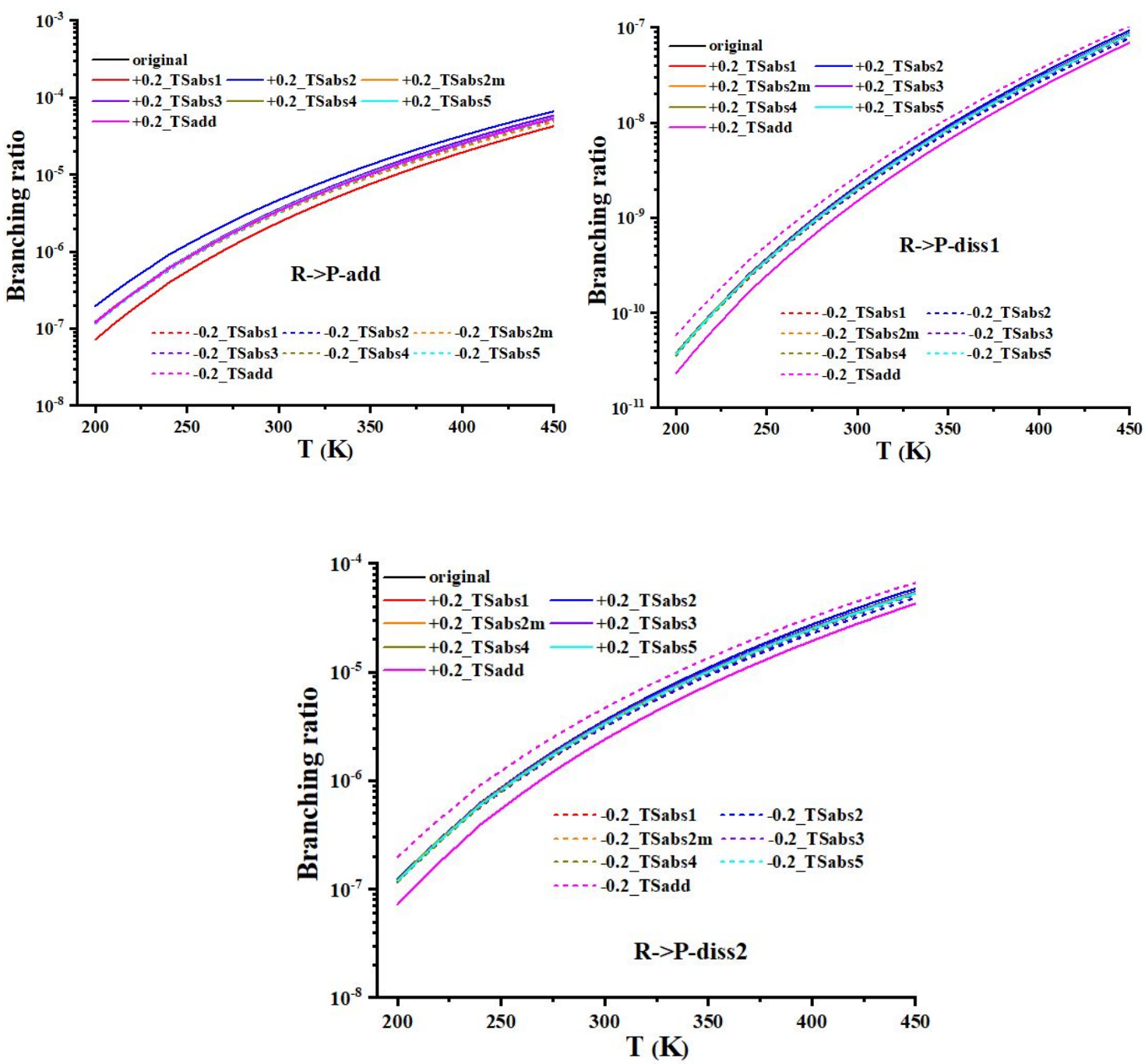

\section{REFERENCES}

1. Zhao, Y.; Truhlar, D. G. The M06 Suite of Density Functionals for Main Group Thermochemistry, Thermochemical Kinetics, Noncovalent Interactions, Excited States, and Transition Elements: Two New Functionals and Systematic Testing of Four M06-class Functionals and 12 Other Functionals. Theor. Chem. Acc. 2008, 120 (1-3), 215-241.

2. Pelucchi, M.; Cavallotti, C.; Faravelli, T.; Klippenstein, S. J. $\mathrm{H}$-abstraction Reactions by $\mathrm{OH}, \mathrm{HO}_{2}, \mathrm{O}, \mathrm{O}_{2}$ and Benzyl Radical Addition to $\mathrm{O}_{2}$ and Their Implications for Kinetic Modelling of Toluene Oxidation. Phys. Chem. Chem. Phys. 2018, 20 (16), 10607-10627.

3. Ji, Y.; Zhao, J.; Terazono, H.; Misawa, K.; Levitt, N. P.; Li, Y.; Lin, Y.; Peng, J.; Wang, Y.; Duan, L.; Pan, B.; Zhang, F.; Feng, X.; An, T.; Marrero-Ortiz, W.; Secrest, J.; Zhang, A. L.; Shibuya, K.; Molina, M. J.; Zhang, R. Reassessing the Atmospheric Oxidation Mechanism of Toluene. Proc Natl Acad Sci U S A 2017, 114 (31), 81698174.

4. Pan, S.; Wang, L. Atmospheric Oxidation Mechanism of m-xylene Initiated by OH Radical. J. Phys. Chem. A 2014, 118 (45), 10778-87.

2426 5. Sirianni, D. A.; Burns, L. A.; Sherrill, C. D. Comparison of Explicitly Correlated Methods for Computing HighAccuracy Benchmark Energies for Noncovalent Interactions. J. Chem. Theory Comput. 2017, 13 (1), 86-99. Harmonic Vibrational Frequencies: Approximate Global Scaling Factors for TPSS, M06, and M11 Functional 
Families Using Several Common Basis Sets. J. Phys. Chem. A 2017, 121 (11), 2265-2273.

2431 7. Korth, M.; Grimme, S. “Mindless” DFT Benchmarking. J. Chem. Theory Comput. 2009, 5, 993-1003.

2432

8. Galano, A.; Francisco-Marquez, M.; Alvarez-Idaboy, J. R. Canolol: a Promising Chemical Agent Against Oxidative Stress. J Phys Chem B 2011, 115 (26), 8590-6.

9. Mayer, P. M.; Parkinson, C. J.; Smith, D. M.; Radom, L. An Assessment of Theoretical Procedures for the Calculation of Reliable Free Radical Thermochemistry: a Recommended New Procedure. J. Chem. Phys. 1998, 108 (2), 604-615.

10. Frisch, M. J.; Trucks, G. W.; Schlegel, H. B.; Scuseria, G. E. Gaussian 16, revision A. 03. Wallingford CT: Gaussian.2016.

11. Neese, F. The Orca Program System, in: 1:73-78. (Ed.). Wires Comput Mol Sci.2012.

12. Neese, F. Software update: The orca program system, version 4.0., 1:e1327. Wires Comput Mol Sci.2018.

13. Bannwarth, C.; Ehlert, S.; Grimme, S. GFN2-xTB-An Accurate and Broadly Parametrized Self-Consistent Tight-Binding Quantum Chemical Method with Multipole Electrostatics and Density-Dependent Dispersion Contributions. J. Chem. Theory Comput. 2019, 15 (3), 1652-1671.

14. Lu, T. Molclus Program, Version 1.9.9, http://www.keinsci.com/research/molclus.html (accessed June 2021).

15. Georgievskii, Y.; Miller, J. A.; Burke, M. P.; Klippenstein, S. J. Reformulation and Solution of the Master Equation for Multiple-well Chemical Reactions. J. Phys. Chem. A 2013, 117 (46), 12146-54.

16. Klippenstein, S. J.; Pande, V. S.; Truhlar, D. G. Chemical Kinetics and Mechanisms of Complex Systems: a Perspective on Recent Theoretical Advances. J. Am. Chem. Soc. 2014, 136 (2), 528-46.

17. Klippenstein, S. J. From Theoretical Reaction Dynamics to Chemical Modeling of Combustion. Proc. Combust. Inst. 2017, 36, 77-111.

18. Georgievskii, Y.; Klippenstein, S. J. available at http://tcg.cse.anl.gov/papr/ codes/mess.html.2016. .

19. Klippenstein, S. J.; Miller, J. A. From the Time-Dependent, Multiple-Well Master Equation to Phenomenological Rate Coefficients. J. Phys. Chem. A 2002, 106 (40), 9267-9277.

20. Miller, J. A.; Klippenstein, S. J. Master Equation Methods in Gas Phase Chemical Kinetics. J. Phys. Chem. A 2006, 110, 10528-10544.

21. Holbrook, K. A.; Pilling, M. J.; Robertson, S. H. Unimolecular Reactions. John Wiley \& Sons: New York, 1996.

22. Eckart, C. The Penetration of a Potential Barrier by Electrons. Phys. Rev. 1930, 35 (11), 1303-1309.

23. Wang, Q. D. Theoretical Studies of Unimolecular Thermal Decomposition Reactions of $n$-hexane and $n-$ hexene Isomers. Computational and Theoretical Chemistry 2017, 1115, 45-55.

24. Zhou, C. W.; Klippenstein, S. J.; Simmie, J. M.; Curran, H. J. Theoretical Kinetics for the Decomposition of iso-Butanol and Related $\left(\mathrm{CH}_{3}\right)_{2} \mathrm{CH}+\mathrm{CH}_{2} \mathrm{OH}$ Reactions. Proc. Combust. Inst. 2013, 34 (1), 501-509.

25. Atkinson, R.; Baulch, D. L.; Cox, R. A.; Hampson, R. F.; Kerr, J. A.; Rossi, M. J.; Troe, J. Evaluated Kinetic and Photochemical Data for Atmospheric Chemistry, Organic Species: Supplement VII. J Phys Chem Ref Data 1999, 28 (2), 191-393.

26. Cord, M.; Husson, B.; Lizardo Huerta, J. C.; Herbinet, O.; Glaude, P. A.; Fournet, R.; Sirjean, B.; Battin-Leclerc, F.; Ruiz-Lopez, M.; Wang, Z.; Xie, M.; Cheng, Z.; Qi, F. Study of the Low Temperature Oxidation of Propane. J. Phys. Chem. A 2012, 116 (50), 12214-28.

27. Atkinson, R.; Aschmann, S. M.; Winer, A. M.; Pitts, J. N. Rate Constants for the Reaction of OH Radicals with a Series of Alkanes and Alkenes at $299 \pm 2$ K. Int. J. Chem. Kinet. 1982, 14, 507-516.

28. Atkinson, R.; Perry, R. A.; Pitts, J. N. J. Rate Constants for the Reaction of Oh Radicals with Ethylene over the Temperature Range $299-425^{\circ} \mathrm{K}$. J. Chem. Phys. 1977, 66, 1197-1201.

29. Overend, R.; Paraskevopoulos, G. Rates of Hydroxyl Radical Reactions. 4. Reactions with Methanol, Ethanol, 1-Propanol, and 2-Propanol at 296 K. J. Chem. Phys. 1977, 67, 674.

30. Lloyd, A. C.; Darnall, K. R.; Winer, A. M.; Pitts, J. N. Relative Rate Constants for Reaction of the Hydroxyl S- 85 
Radical with a Series of Alkanes, Alkenes, and Aromatic Hydrocarbons. J. Phys. Chem. 2002, 80 (8), $789-794$. 31. Vakhtin, A. B.; Murphy, J. E.; Leone, S. R. Low-temperature Kinetics of Reactions of OH Radical with Ethene, Propene, and 1-Butene. J. Phys. Chem. A 2003, 107 (47), 10055-10062.

32. Tully, F. P.; Goldsmith, J. E. M. Kinetic Study of the Hydroxyl Radical-propene Reaction. Chem. Phys. Lett. 1985, 116 (4), 345-352.

33. Izsa, R.; Szo"ri, M.; Knowles, P. J.; Viskolcz, B. High Accuracy ab Initio Calculations on Reactions of OH with 1-Alkenes. The Case of Propene. J. Chem. Theory Comput. 2009, 5, 2313-2321.

34. Ravishankara, A. R.; Wagner, S.; Fischer, S.; Smith, G.; Schiff, R.; Watson, R. T.; Tesi, G.; Davis, D. D. A Kinetics Study of the Reactions of $\mathrm{OH}$ with Several Aromatic and Olefinic Compounds. Int. J. Chem. Kinet. 1978, 105, 783804.

35. Peirone, S.; Nieto, J. D.; Cometto, P. M.; da Silva Barbosa, T.; Bauerfeldt, G. F.; Arbilla, G.; Lane, S. I. Comparative Kinetics of the 3-buten-1-ol and 1-butene Reactions with $\mathrm{OH}$ Radicals: a Density Functional Theory/RRKM Investigation. J. Phys. Chem. A 2015, 119 (13), 3171-80.

36. Chen, L.; Huang, Y.; Xue, Y.; Jia, Z.; Wang, W. Atmospheric Oxidation of 1-butene Initiated by OH Radical: Implications for Ozone and Nitrous Acid Formations. Atmos. Environ. 2021, 244, 118010.

37. Atkinson, R.; Pitts, J. N. Kinetics of the Reaction of $\mathrm{O}\left({ }^{3} \mathrm{P}\right)$ Atoms and $\mathrm{OH}$ Radicals with 2-methyl-2-butene. J. Chem. Phys. 1978, 68, 2992-2994.

38. Iglesias-Arteaga, M. A.; Tejero, T. N.; Bauerfeldt, G. F. Multipath Kinetics of the Reaction of OH Radical with 1-pentene. Arkivoc 2020, 2020 (2), 20-33.

39. Nishino, N.; Arey, J.; Atkinson, R. Rate Constants for the Gas-Phase Reactions of OH Radicals with a Series of C6-C14 Alkenes at $299 \pm 2$ K. J. Phys. Chem. A 2009, 113, 852-857.

40. Zhang, W.; Du, B. Theoretical Investigation on the Reaction between $\mathrm{OH}$ Radical and 4,4-dimethyl-1pentene in the Presence of $\mathrm{O}_{2}$. J. Phys. Chem. A 2015, 119 (17), 4065-72.

41. Yin, G. Y.; Hu, E. J.; Yang, F. Y.; Ku, J. F.; Huang, Z. H. Kinetics of H Abstraction and Addition Reactions of 2,4,4-trimethyl-1-pentene by OH Radical. Fuel 2017, 210, 646-658.

42. Yin, G. Y.; Hu, E. J.; Gao, Z. H.; Yang, F. Y.; Huang, Z. H. Kinetics of H Abstraction and Addition Reactions of 2,4,4-trimethyl-2-pentene by OH Radical. Chem. Phys. Lett. 2018, 696, 125-134.

43. Barbosa Tda, S.; Peirone, S.; Barrera, J. A.; Abrate, J. P.; Lane, S. I.; Arbilla, G.; Bauerfeldt, G. F. Rate Coefficients for the Reaction of $\mathrm{OH}$ Radicals with cis-3-hexene: an Experimental and Theoretical Study. Phys. Chem. Chem. Phys. 2015, 17 (14), 8714-22.

44. Yang, F.; Deng, F.; Pan, Y.; Zhang, Y.; Tang, C.; Huang, Z. Kinetics of Hydrogen Abstraction and Addition Reactions of 3-Hexene by OH Radicals. J. Phys. Chem. A 2017, 121 (9), 1877-1889.

45. Aschmann, S. M.; Atkinson, R. Rate Constants for the Gas-phase Reactions of OH Radicals with E-7tetradecene, 2-methyl-1-tridecene and the C(7)-C(14) 1-alkenes at $295 \pm 1$ K. Phys. Chem. Chem. Phys. 2008, 10 (28), 4159-64.

46. Iuga, C.; Alvarez-Idaboy, J. R.; Reyes, L.; Vivier-Bunge, A. Can a Single Water Molecule Really Catalyze the Acetaldehyde $+\mathrm{OH}$ Reaction in Tropospheric Conditions? J. Phys. Chem. Lett. 2010, 1, 3112-3115.

47. Semmes, D. H.; Ravishankara, A. R.; Gumpperkins, C. A.; Wine, P. H. Kinetics of the Reactions of Hydroxyl Radical with Aliphatic-Aldehydes. Int. J. Chem. Kinet. 1985, 17 (3), 303-313.

48. Niki, H.; Maker, P. D.; Savage, C. M.; Breitenbach, L. P. Relative Rate Constants for the Reaction of Hydroxyl Radical with Aldehydes. J. Phys. Chem. 1978, 82 (2), 132-134.

49. Kerr, J. A.; Sheppard, D. W. Kinetics of the Reactions of Hydroxyl Radicals with Aldehydes Studied Under Atmospheric Conditions. Environ. Sci. Technol. 1981, 15 (8), 960-3.

50. Audley, G. J.; Baulch, D. L.; Campbell, I. M. Gas-phase Reactions of Hydroxyl Radicals with Aldehydes in Flowing $\mathrm{H}_{2} \mathrm{O}_{2}+\mathrm{NO}_{2}+\mathrm{CO}$ Mixtures. J. Chem. Soc., Faraday Trans. 1981, 77, 2541-2549.

51. Iuga, C.; Ignacio Sainz-Díaz, C.; Vivier-Bunge, A. On the OH Initiated Oxidation of C2-C5 Aliphatic Aldehydes S-86 
2522 in the Presence of Mineral Aerosols. Geochim. Cosmochim. Acta 2000, 74 (12), 3587-3597.

2523 52. Stemmler, K.; Mengon, W.; Kerr, J. A. Hydroxyl-radical-initiated Oxidation of Isobutyl Isopropyl Ether Under

2524

2525

2526

2527

2528

2529

2530

2531

2532

2533

2534

2535

2536

2537

2538

2539

2540

2541

2542

2543

2544
Laboratory Conditions Related to the Troposphere. Product Studies and Proposed Mechanism. J Chem Soc Faraday T 1997, 93 (16), 2865-2875.

53. Dóbé, S.; Khachatryan, L. A.; Bérces, T. Kinetics of Reactions of Hydroxyl Radicals with a Series of Aliphatic Aldehydes. Ber. Bunsen Ges. Phys. Chem. 1989, 93, 847-852

54. D'Anna, B.; Andresen, W.; Gefen, Z.; Nielsen, C. J. Kinetic Study of $\mathrm{OH}$ and $\mathrm{NO}_{3}$ Radical Reactions with 14 Aliphatic Aldehydes. Phys. Chem. Chem. Phys. 2001, 3 (15), 3057-3063.

55. Papagni, C.; Arey, J.; Atkinson, R. Rate Constants for the Gas-phase Reactions of a Series of C3-C6 Aldehydes with $\mathrm{OH}$ and $\mathrm{NO}_{3}$ Radicals. Int. J. Chem. Kinet. 2000, 32, 79-84.

56. Albaladejo, J.; Ballesteros, B.; Jimenez, E. A PLP-LIF Kinetic Study of the Atmospheric Reactivity of a Series of C4-C7 Saturated and Unsaturated Aliphatic Aldehydes with OH. Atmos. Environ. 2002, 36, 3231-3239.

57. Atkinson, R. Estimations of $\mathrm{OH}$ Radical Rate Constants from $\mathrm{H}-$ Atom Abstraction from $\mathrm{C}-\mathrm{H}$ and $\mathrm{O}-\mathrm{H}$ Bonds over the Temperature Range 250-1000 K. Int. J. Chem. Kinet. 1986, 18, 555-568.

58. Atkinson, R. A Structure-activity Relationship for the Estimation of Rate Constants for the Gas-phase Reactions of $\mathrm{OH}$ Radicals with Organic Compounds. Int. J. Chem. Kinet. 1987, 19, 799-828.

59. Kwok, E. S. C.; Atkinson, R. Estimation of Hydroxyl Radical Reaction Rate Constants for Gas-phase Organic Compounds Using a Structure-reactivity Relationship: an Update. Atmos. Environ. 1995, 29, 1685-1695.

60. Wu, X.; Huang, C.; Niu, S.; Zhang, F. New Theoretical Insights into the Reaction Kinetics of Toluene and Hydroxyl Radicals. Phys. Chem. Chem. Phys. 2020, 22 (39), 22279-22288.

61. Wu, X.; Hou, Q.; Huang, J.; Chai, J.; Zhang, F. Exploring the OH-initiated Reactions of Styrene in the Atmosphere and the Role of Van der Waals Complex. Chemosphere 2021, 282, 131004. 